\title{
Modellierung der vegetativen Produktivität zur Bewertung der Landdegradation im ariden und semi-ariden südlichen Afrika
}

\author{
Dissertation \\ zur Erlangung des mathematisch-naturwissenschaftlichen Doktorgrades \\ „Doctor rerum naturalium“ \\ der Georg-August-Universität Göttingen \\ im Promotionsprogramm Geowissenschaften / Geographie \\ der Georg-August University School of Science (GAUSS) \\ vorgelegt von \\ Markus Niklaus
}

aus Schweinfurt

Göttingen 2013 
Referent: Prof. Dr. Martin Kappas

Koreferent: Prof. Dr. Gerhard Gerold

Tag der mündlichen Prüfung: 16.12.2013 




\section{Inhaltsverzeichnis}

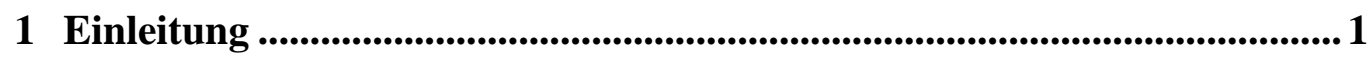

2 Methodenbeschreibung............................................................................................ 7

2.1 Landdegradation in Trockengebieten ................................................. 7

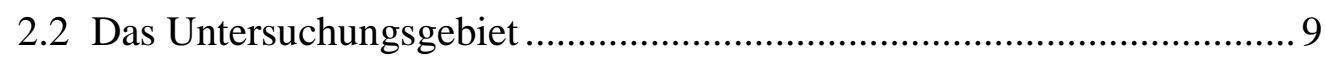

2.3 Das Vegetationsmodell BETHY/DLR ................................................ 14

2.3.1 Die grundlegenden Funktionen ............................................. 14

2.3.2 Die Photorespiration ............................................................ 20

2.3.3 Die Bruttoprimärproduktion .................................................. 20

2.3.4 Die Nettoprimärproduktion ................................................ 27

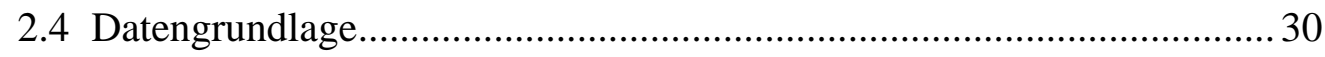

2.4.1 Meteorologischer Antrieb ...................................................... 30

2.4.2 Der Blattflächenindex ........................................................ 38

2.4.3 Die Landnutzungsklassifikation .......................................... 61

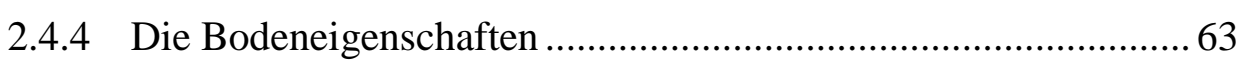

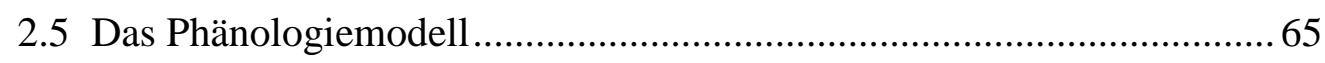

3 Modellsensitivität..................................................................................... 77

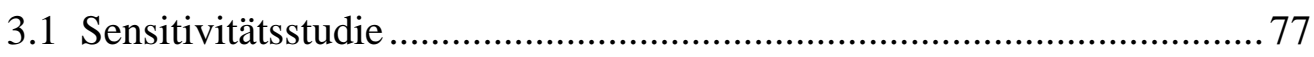

3.1.1 Die Höhe über Normalnull ................................................... 79

3.1.2 Der Blattflächenindex ......................................................... 80

3.1.3 Die Temperatur ................................................................... 81

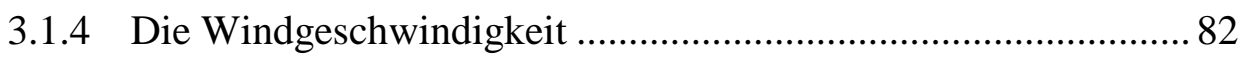

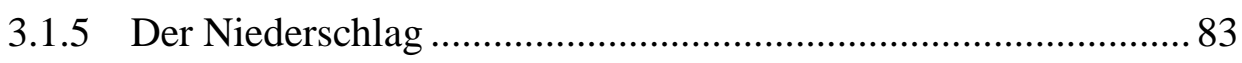

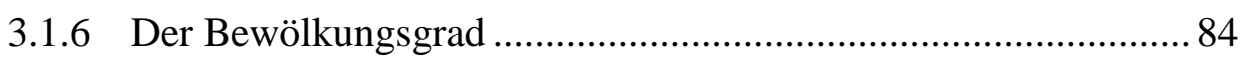

3.1.7 Die Landbedeckungsklassifikation ......................................... 85

4 Die Modellergebnisse..................................................................................89

4.1 Biomassemessungen im Krüger-Nationalpark ...................................... 89

4.2 Messungen des Kohlenstoffflusses durch Eddy-Kovarianz-Türme ........ 94

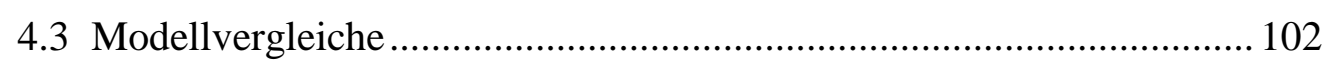

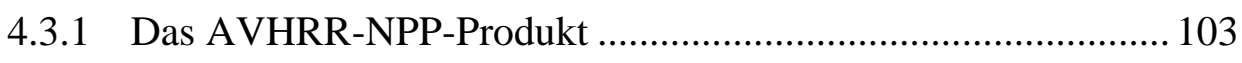

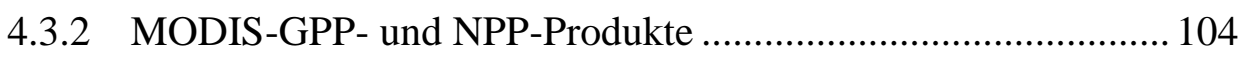

5 Der Degradationsindex ................................................................................... 107

5.1 Trends in der Vegetationsproduktivität ........................................... 107 
5.2 Anomalien in klimatischen Zeitreihen................................................. 110

5.3 Indexierung der Landdegradation .......................................................113

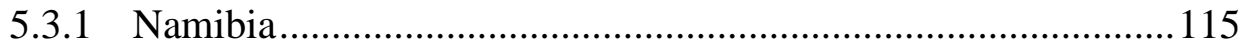

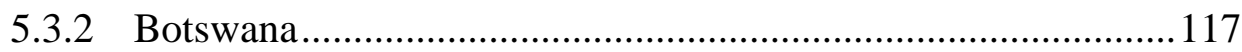

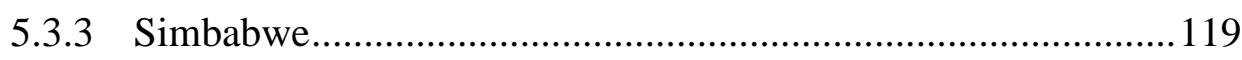

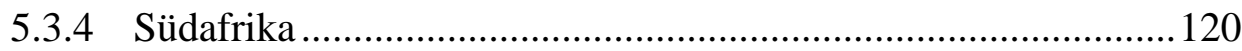

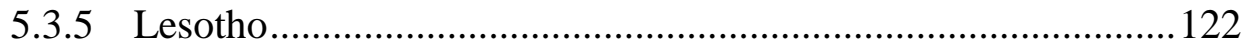

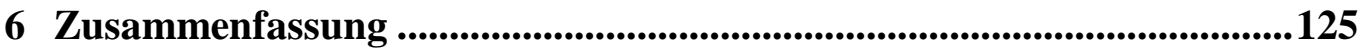

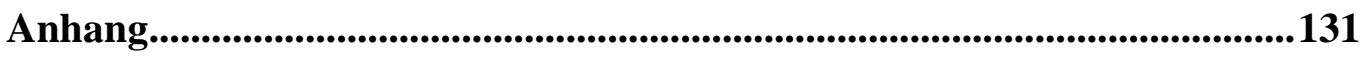

Anhang I. Vergleich der MODIS-GPP- und NPP-Produkte...................131

Literaturverzeichnis ..............................................................................................135 


\section{Abbildungsverzeichnis}

Abbildung 2.1: Köppen-Geiger Klassifikation der Klimatypen im Arbeitsgebiet des südlichen Afrika. Die Beschreibung der Legende erfolgt in Tabelle 2.1 und Tabelle 2.2... 10

Abbildung 2.2: Klimatische Eigenschaften des Arbeitsgebietes berechnet aus Zeitreihen von ECMWF Parametern gemittelt über die jährlichen Werte des Zeitraumes 1989 bis 2010. Jahressumme des Niederschlags (a), Jahreseintrag an photosynthetisch aktiver Strahlung (PAR) (b), Jahresmittelwert der Temperatur (c) und Höhenprofil (d)............... 12

Abbildung 2.3: Verteilung der 760 meteorologischen Messstationen des ARC über Südafrika. 31

Abbildung 2.4: Verteilung der Korrelationskoeffizienten der einzelnen ARC-Messstationen (a) und das Streudiagramm aller Messwerte (b) für den Niederschlag. 32

Abbildung 2.5: Streudiagramm der Monatssummen des Niederschlags aller ARCMessstationen gegen die Werte des ECMWF.

Abbildung 2.6: Verteilung der Korrelationskoeffizienten der einzelnen ARC-Messstationen (a) und das Streudiagramm aller Messwerte (b) für das Tagesminimum der Temperatur. . 34

Abbildung 2.7: Verteilung der Korrelationskoeffizienten der einzelnen ARC-Messstationen (a) und das Streudiagramm aller Messwerte (b) für das Tagesmaximum der Temperatur.. 34

Abbildung 2.8: Verteilung der Korrelationskoeffizienten der einzelnen ARC-Messstationen (a) und das Streudiagramm aller Messwerte (b) für die Windgeschwindigkeit. 35

Abbildung 2.9: Verteilung der Korrelationskoeffizienten der einzelnen ARC-Messstationen (a) und das Streudiagramm aller Messwerte (b) für die PAR.

Abbildung 2.10: Streudiagramm der Monatssummen der PAR aller ARC Messstationen gegen die Werte des ECMWF. 38

Abbildung 2.11. Globale Kachelung des SPOT-VGT LAI Produkts. Nur für grün markierte Kacheln wurden LAI Werte prozessiert. (Quelle: geoland2, Baret et al., 2010). 39

Abbildung 2.12. Zerlegung einer Zeitreihe durch die Harmonische Analyse in fünf harmonische Einzelschwingungen. 41

Abbildung 2.13. Ergebnis der ursprünglichen Version der HA angewandt auf eine LAIZeitserie mit Datenlücken (dargestellt mit Wert -1). Die Zeitserie zeigt ein als sommergrüner Laubwald klassifiziertes Pixel in Massachusetts, USA, für das Jahr 2003. 42

Abbildung 2.14. Mittlere Phänologie der Vegetationsklasse ,sommergrüner Laubwald' für das Jahr 2003 in der Kachel ,H10V4'. Die Fehlerbalken geben die Standardabweichung der einzelnen Werte an. 43 
Abbildung 2.15. Ergebnis der modifizierten HA (durchgezogene Linie) angewandt auf die Originaldaten (Kreuze) des bereits in Abbildung 2.13 gezeigten Mischwaldpixels. Werte von -1 bezeichnen Datenlücken. Die Dreiecke stellen die mittlere Phänologie der zugehörigen Landbedeckungsklasse dar.

Abbildung 2.16. Vergleich von repräsentativen SPOT-VGT-Kacheln vor (erste Reihe, SPOT-VGT) und nach (zweite Reihe, HA) der Anwendung der Zeitreihenanalyse. Die Kacheln sind: Links, H7V5, Nordamerika; Mitte, H19V4, Europa; Rechts, H29V4, Asien. Die Kacheln und entsprechende Zeitpunkte sind oberhalb der Abbildung beschrieben. C-xx steht für das jeweilige Komposit des Jahres. Weiße Flächen stellen fehlende LAI-Werte bzw. Wasserpixel dar. 48

Abbildung 2.17. Vergleich von repräsentativen SPOT-VGT-Kacheln vor (erste Reihe, SPOT-VGT) und nach (zweite Reihe, HA) der Anwendung der Zeitreihenanalyse. Die Kacheln sind: Links, H18V7, Afrika; Mitte, H29V12, Australien; Rechts, H12V8, Südamerika. Die Kacheln und entsprechende Zeitpunkte sind oberhalb der Abbildung beschrieben. C-xx steht für das jeweilige Komposit des Jahres. Weiße Flächen stellen fehlende LAI-Werte bzw. Wasserpixel dar.

Abbildung 2.18. Differenz (SPOT-VGT - HA) (obere Reihe), NRMSE (mittlere Reihe) und Anteil an aufgefüllten Werten (untere Reihe) für die entsprechenden Jahre bestimmt für die in Abbildung 2.16 gezeigten Kacheln. Weiße Pixel zeigen Gebiete, für die keine HA berechnet wurde bzw. Gebiete für die keine Lücken aufgefüllt wurden. Die verschiedenen Kacheln und Jahre sind über den Abbildungen angegeben. 50

Abbildung 2.19. Differenz (SPOT-VGT - HA) (obere Reihe), NRMSE (mittlere Reihe) und Anteil an aufgefüllten Werten (untere Reihe) für die entsprechenden Jahre bestimmt für die in Abbildung 2.17 gezeigten Kacheln. Weiße Pixel zeigen Gebiete, für die keine HA berechnet wurde bzw. Gebiete für die keine Lücken aufgefüllt wurden. Die verschiedenen Kacheln und Jahre sind über den Abbildungen angegeben. 51

Abbildung 2.20. LAI-Zeitreihe für ein Pixel in der Übergangszone zur Sahel Wüste 52

Abbildung 2.21: GLC2000 (a), LAI (18. Komposit für 2001) (b) und zugehöriger NRMSE (c) für den Plattensee (Ungarn, mittig) und den Neusiedlersee (Österreich, oben links im Bild). Schwarze Linien zeigen die SRTM-Wassermaske.

Abbildung 2.22: Globale Karten (a) des LAI des 18. Komposits und (b) des zugehörigen NRMSE für 2001. Weiße Pixel repräsentieren Werte für die keine Daten zur Verfügung stehen. .55

Abbildung 2.23. Streudiagramm des direkten Vergleichs von SPOT-VGT-Originaldaten (blaue Rauten) und neu prozessierten SPOT-VGT-Daten (rote Dreiecke) mit den Werten der LAI-Referenzkarten.

Abbildung 2.24 Abbildung 2.20. Zeitlicher Verlauf der ursprünglichen (schwarze Kreuze) und der neu prozessierten (durchgezogene grüne Linie) Spot-VGT-Daten für Ackerland (a) 
und Grasland (b). Blaue Dreiecke bezeichnen die Referenz-LAI-Werte. Die Stationsdetails sind in den Diagrammen beschrieben.

Abbildung 2.25 Zeitlicher Verlauf der ursprünglichen (schwarze Kreuze) und der neu prozessierten (durchgezogene grüne Linie) Spot-VGT-Daten für Mischwald (a) und immergrüne Wälder (b). Blaue Dreiecke bezeichnen die Referenz-LAI-Werte. Die Stationsdetails sind in den Diagrammen beschrieben. 60

Abbildung 2.26: Verteilung der Vegetationsklassen nach GLC2000 innerhalb des Arbeitsgebietes.

Abbildung 2.27: PDF (a) und Verteilungsfunktion (b) der Temperatur für ein Pixel im Arbeitsgebiet.

Abbildung 2.28: PDF (a) und Verteilungsfunktion (b) der PAR für ein Pixel im Arbeitsgebiet.

Abbildung 2.29: Mittlere Werte des NRMSE für die Haupt-Vegetationstypen des Arbeitsgebietes. Abkürzungen der Vegetationstypen sind: immergrüner Laubwald (ILW), sommergrüner Laubwald (SLW), sommergrünes Buschland (SB), Grasland (GL), Sumpfland (SL), Kulturpflanzen (KL).

Abbildung 2.30: Vergleich der Zeitreihen aus den Originaldaten des SPOT-LAI und durch das Phänologiemodell berechnet. Gezeigt sind Beispiele der sechs häufigsten Vegetationstypen innerhalb des Arbeitsgebietes: (a) immergrüner Laubwald, gemäßigt (Klasse 3); (b) sommergrüner Laubwald, gemäßigt (Klasse 4); (c) sommergrünes Buschland (Klasse 8); (d) Grasland, C4, kurz (Klasse 11); (e) Sumpfland (Klasse 14); (f) Kulturpflanzen (Klasse 15).

Abbildung 3.1: Mittlere Abweichung der Modellergebnisse GPP (links) und NPP (rechts) vom Referenzwert bei der Variation der Höhe über dem Meeresspiegel.

Abbildung 3.2: Mittlere Abweichung der Modellergebnisse GPP (links) und NPP (rechts) vom Referenzwert bei der Variation des Blattflächenindexes.

Abbildung 3.3: Mittlere Abweichung der Modellergebnisse GPP (links) und NPP (rechts) vom Referenzwert bei der Variation der Temperatur.

Abbildung 3.4: Mittlere Abweichung der Erhaltungsatmung $R_{m}$ vom Referenzwert bei der Variation der Temperatur.

Abbildung 3.5: Mittlere Abweichung der Modellergebnisse GPP (links) und NPP (rechts) vom Referenzwert bei der Variation der Windgeschwindigkeit.

Abbildung 3.6: Mittlere Abweichung der Modellergebnisse GPP (links) und NPP (rechts) vom Referenzwert bei der Variation des Niederschlags. 
Abbildung 3.7: Mittlere Abweichung der Modellergebnisse GPP (links) und NPP (rechts) vom Referenzwert bei der Variation des Bewölkungsgrades.

Abbildung 3.8: Mittlere Abweichung der Modellergebnisse GPP (oben) und NPP (unten) vom Referenzwert bei Veränderung der Vegetationsklassifikation entsprechend der in Tabelle 2.4 definierten Vegetationstypen.

Abbildung 4.1: Lage des Krüger-Nationalparks in Südafrika und Verteilung der Messgebiete innerhalb des Parks. Die Farbskala zeigt die Höhe über Normalnull, links sind die Nationalparks in blau angezeigt, rechts die Orte der Messgebiete als offene Kreise.... 90

Abbildung 4.2: Streudiagramm der akkumulierten NPP aus dem Modell und den Differenzen der Biomassemessungen im Krüger-Nationalpark. Die Fehlerbalken zeigen die Standardabweichung der Messdaten an. Die gestrichelte Linie markiert die 1:1-Gerade... 91

Abbildung 4.3: Korrelationskoeffizient der einzelnen Stationen zwischen den Messungen der Biomasse und den modellierten NPP-Summen über die Wachstumsphase.

Abbildung 4.4: Messturm der Station Mongu (links) und die Anordnung der Messgeräte an der Spitze des Messturms (rechts), mit Ultraschallanemometer (rechtes Bild links), Infrarot Gasanalysator für $\mathrm{CO}_{2}$ und $\mathrm{H}_{2} \mathrm{O}$ (rechtes Bild Mitte) und Gasanalysator für $\mathrm{CH}_{4}$ (rechtes Bild rechts). (Bilder: fluxnet.ornl.gov und licor.com). 95

Abbildung 4.5: Lage der Stationen Mongu, Maun, Malopeni und Skukuza innerhalb des Modellierungsgebietes. 96

Abbildung 4.6: Vergleich der Modellergebnisse (rot) der GPP mit aus Eddy-KovarianzMessungen abgeleiteten Werten (schwarz) an vier Stationen im Untersuchungsgebiet. Links oben Malopeni, Krüger-Nationalpark, Südafrika; rechts oben Maun, Botswana; links unten Mongu in Simbabwe; rechts unten Skukuza, Krüger-Nationalpark, Südafrika. ....... 97

Abbildung 4.7: Streudiagramme von täglichen Modellergebnissen und Messwerten an den vier FLUXNET-Stationen. Werte unter $0.5 \mathrm{gC} \mathrm{m}^{-2} \mathrm{~d}^{-1}$ (rot) werden bei dem Vergleich nicht berücksichtigt. Die gestrichelte, graue Linie kennzeichnet die 1:1-Gerade. .98

Abbildung 4.8: Streudiagramme der Monatssummen aus den Modellergebnissen und den Stationsmessungen. Die gestrichelte, graue Linie kennzeichnet die 1:1-Gerade. 99

Abbildung 4.9: Verteilung der administrativen Distrikte der Nationen im südlichen Afrika, zur Bestimmung der NPP- und GPP-Summen für den Modellvergleich. 102

Abbildung 4.10: Vergleich der NPP-Ergebnisse aus den Modellen BETHY/DLR und GloPEM für 1999 (links) und 2000 (rechts). Innerhalb der Diagramme ist jeweils der Bereich niedriger Werte dargestellt. 103 
Abbildung 4.11: Vergleich der Modellergebnisse aus BETHY/DLR und MODIS für die GPP (links) und die NPP (rechts). Die gestrichelte Linie stellt die 1:1-Linie dar. Innerhalb der Abbildungen ist der Bereich kleinerer Werte vergrößert dargestellt.

Abbildung 5.1: Zeitreihe der NPP als Tageswerte (blaue Linie) und Jahressummen (rote Kreise) über die Vegetationsperioden von 1999 bis 2010 . 108

Abbildung 5.2: Karte der berechneten NPP-Trends für das gesamte Arbeitsgebiet aus den NPP-Summen der einzelnen Vegetationsperioden (a) und der zugehörigen Korrelation des linearen Trends (b).

Abbildung 5.3: Zeitreihen des Niederschlags (a) und der mittleren Temperatur (b) über die Vegetationsperioden von 1999 bis 2010. Blaue Linien zeigen die Tageswerte, rote Kreise den Jahresniederschlag bzw. die mittlere Jahrestemperatur mit dem Trend über den gesamten Zeitraum (schwarze Linie). 110

Abbildung 5.4: Relativer Trend des mittleren Jahresniederschlags (a) bezüglich des Wertes zu Beginn des Betrachtungszeitraumes 99/01-09/10 mit der zugehörigen Korrelation des linearen Fits (b).

Abbildung 5.5: Relativer Trend der mittleren Temperatur (a) bezüglich des Wertes zu Beginn des Betrachtungszeitraumes 99/01-09/10 mit der zugehörigen Korrelation des linearen Fits (b).

Abbildung 5.6: Degradationsindex des Arbeitsgebietes mit den Unterteilungen in die Kategorien A: nicht-klimatisch bedingt (grün), B: signifikanter Rückgang des Niederschlags (braun), C: signifikante Temperaturzunahme (rot) und D: kombinierte Temperaturzunahme und Niederschlagsrückgang (blau). Die Zahlenzusätze bezeichnen den Grad des NPP-Rückgangs: 1: 0 bis $25 \%$; 2: $25 \%$ bis $50 \%$; 3: $50 \%$ bis $75 \%$; 4: über $75 \%$.

Abbildung 5.7: Degradationsindex für Namibia mit den Vergleichsregionen (schwarze Markierungen) 116

Abbildung 5.8: Degradationsindex für Botswana mit den Vergleichsregionen (schwarze Markierungen).

Abbildung 5.9: Degradationsindex für Simbabwe mit der Vergleichsregion Buhera (schwarze Markierung). In der Region sind die von Mambo und Archer (2007) als degradiert identifizierten Gebiete markiert (rote Markierungen). 120

Abbildung 5.10: Degradationsindex für Südafrika mit den Vergleichsregionen (schwarze Markierungen).

Abbildung 5.11: Degradationsindex für Lesotho mit den einzelnen Regionen. 
Abbildung A.1: Vergleich der Modellergebnisse aus BETHY/DLR und MODIS für die GPP (links) und die NPP (rechts) für die Jahre 2000 (oben), 2001 (Mitte) und 2002 (unten). Die gestrichelte Linie stellt die 1:1-Linie dar. Innerhalb der Abbildungen ist der Bereich kleinerer Werte vergrößert dargestellt.

Abbildung A.2: Vergleich der Modellergebnisse aus BETHY/DLR und MODIS für die GPP (links) und die NPP (rechts) für die Jahre 2003 (oben), 2004 (Mitte) und 2005 (unten). Die gestrichelte Linie stellt die 1:1-Linie dar. Innerhalb der Abbildungen ist der Bereich kleinerer Werte vergrößert dargestellt.

Abbildung A.3: Vergleich der Modellergebnisse aus BETHY/DLR und MODIS für die GPP (links) und die NPP (rechts) für die Jahre 2006 (oben), 2007 (Mitte) und 2008 (unten). Die gestrichelte Linie stellt die 1:1-Linie dar. Innerhalb der Abbildungen ist der Bereich kleinerer Werte vergrößert dargestellt.

Abbildung A.4: Vergleich der Modellergebnisse aus BETHY/DLR und MODIS für die GPP (links) und die NPP (rechts) für die Jahre 2009 (oben) und 2010 (unten). Die gestrichelte Linie stellt die 1:1-Linie dar. Innerhalb der Abbildungen ist der Bereich kleinerer Werte vergrößert dargestellt. 134 


\section{Tabellenverzeichnis}

Tabelle 2.1: Unterteilung der Hauptklimazonen (A: äquatorial, B: arid, C: warm-gemäßigt) in Klimatypen des Arbeitsgebietes unter Berücksichtigung der Niederschlagscharakteristika (m: monsunal, S: Savanne, W: Wüste, s: sommertrocken, w: wintertrocken und f: feuchtgemäßigt) nach Köppen und Geiger (Köppen, 1900; Geiger, 1954; Kottek et al., 2006).

Tabelle 2.2: Unterteilung der Temperaturklassen im Arbeitsgebiet nach Köppen und Geiger (Köppen, 1900; Geiger, 1954; Kottek et al., 2006).

Tabelle 2.3: Unterscheidung der Albedo für feuchten $\left(\rho_{b, n}\right)$ und trockenen $\left(\rho_{b, t}\right)$ Boden in helle, mittlere und dunkle Bodentypen.

Tabelle 2.4: Vegetationstypen des Modells mit den spezifischen Parametern: $V_{m}$ : maximale Karboxylierungsrate bei $25^{\circ} \mathrm{C} \quad\left[\mu \mathrm{mol}\left(\mathrm{CO}_{2}\right) \quad \mathrm{m}^{-2} \mathrm{~s}^{-1}\right]$, für $\mathrm{C} 3: J_{m}$ : maximale Elektronentransportrate bei $25^{\circ} \mathrm{C}\left[\mu \mathrm{mol}\left(\mathrm{CO}_{2}\right) \mathrm{m}^{-2} \mathrm{~s}^{-1}\right]$ für $\mathrm{C} 4: \mathrm{k}: \mathrm{CO}_{2}$ Spezifität bei $25^{\circ} \mathrm{C}$ $\left[\mathrm{mmol}\left(\mathrm{CO}_{2}\right) \mathrm{m}^{-2} \mathrm{~s}^{-1}\right]$, h: Vegetationshöhe $[\mathrm{m}], \mathrm{dr}$ : Wurzeltiefe [m], C4: C4-Mechanismus, ansonsten $\mathrm{C} 3$.

Tabelle 2.5: Variablen zur Berechnung der Photosyntheserate nach Farquhar et al. (1980) (C3) und Collatz et al. (1992) (C4). $O_{x}$ ist der Partialdruck von Sauerstoff, $K_{C}$ und $K_{O}$ die Michaelis-Menten Konstanten für Kohlenstoff und Sauerstoff. $V_{m}$ ist die maximale Karboxylierungsrate und $k$ die $\mathrm{CO}_{2}$-Spezifität mit den pflanzenspezifischen Werten aus Tabelle 2.4. $E$ gibt die Werte der Aktivierungsenergie in $\mathrm{J} \mathrm{mol}^{-1}$ an. 22

Tabelle 2.6. Eigenschaften der Validierungsgebiete (insgesamt 24) und deren Referenzkarten (insgesamt 43). Die Abkürzungen der Überschrift sind: „Lat“ für Latitude, „Lon“ für Longitude, „LC“ für Landbedeckung (engl.: land cover). „MAP“ sind die Mittelwerte der LAI-Referenzkarten, „CYC“ die Mittelwerte der SPOT-VGT-Pixel und „HA“ die Mittelwerte der neu prozessierten SPOT-VGT-Pixel. Die Landbedeckungsklassen sind: „ILW“ für immergrünen Laubwald „MW“ für Mischwald, „INW“ für immergrüner Nadelwald, „KP“ für Kulturpflanze und „GL“ für Grasland. ..... 56

Tabelle 2.7: Globale Landbedeckungsklassen der GLC2000 nach dem LCCS von FAO und UNEP. 61

Tabelle 2.8: Übersetzung der GLC2000-Vegetationstypen in Primär- (PK) und Sekundärklassen (SK) der BETHY/DLR-Vegetationstypen mit der jeweiligen Gewichtung $(g)$.

Tabelle 2.9: Modellparameter die zur Kalibrierung variiert werden, mit dem Wertebereich [min; max] und den Variationsschritten $\Delta$.

Tabelle 2.10: Nach der Kalibrierung festgelegte Werte der Modellparameter für die HauptVegetationstypen des Arbeitsgebietes. Abkürzungen der Vegetationstypen sind: 
immergrüner Laubwald (ILW), sommergrüner Laubwald (SLW), sommergrünes Buschland (SB), Grasland (GL), Sumpfland (SL), Kulturpflanzen (KP).

Tabelle 3.1: Eigenschaften der FLUXNET-Stationen mit geographischer Breite (Lat), geographischer Länge (Lon), der Landbedeckungsklassifikation (LCC) und der Höhe (h). Die Klassifikation wird unterschieden in sommergrünen Laubwald (SLW), Grasland (GL) und sommergrünes Buschland (SBL). 78

Tabelle 3.2: Langzeitmittel der einzelnen Parameter der Sensitivitätsanalyse für die Jahressummen des Niederschlags $(P)$ und für die Jahresmittelwerte der minimalen $\left(T_{\text {min }}\right)$, der maximalen $\left(T_{\max }\right)$ und der mittleren Tagestemperatur $\left(T_{m i t}\right)$, des LAI, der Windgeschwindigkeit $(u)$ und dem Anteil an erreichter potentieller PAR $\left(f_{P A R}\right) \ldots \ldots \ldots \ldots \ldots \ldots . . . . .78$

Tabelle 3.3: Modellergebnisse der GPP und der NPP $\left[\mathrm{gC} \mathrm{m}^{-2} \mathrm{Jahr}^{-1}\right]$ an den vier Stationen mit unveränderten Eingangsparametern als Referenzwerte.

Tabelle 5.1: Klassifizierung des Degradationsindexes mit der Farbkodierung aus Abbildung 5.6. $\triangle \mathrm{NPP}$ ist der NPP-Trend, $\Delta \mathrm{P}$ der Niederschlags-Trend und $\Delta \mathrm{T}$ der Temperaturtrend über den Betrachtungszeitraum 99/00 bis 09/10. 114 


\section{Abkürzungsverzeichnis}

ARC

APAR

ATP

AVHRR

BETHY-Mode

BOREAS

BRDF

CASA

CCDAS

CGER

CYCLOPES

DEM

DFD

DGVM

DKRZ

DLR

DPM

ECHAM

ECMWF

EK

EPIC

ERA

FAO
Agricultural Research Council

Absorbed Photosynthetically Active Radiation

Adenosin-Tri-Phosphat

Advanced Very High Resolution Radiometer

Biosphere Energy Transfer HYdrology Model

Boreal Ecosystem-Atmosphere Study

Bidirectional Reflectance Distribution Function

Carnegie-Ames-Stanford Approach

Carbon Cycle Data Assimilation System

Commission on Geosciences, Environment and Resources

Carbon cycle and change in land observational products from an ensemble of satellites

Digital Elevation Model

Deutsches Fernerkundungsdatenzentrum

Dynamic Global Vegetation Model

Deutsches Klimarechenzentrum

Deutsches Zentrum für Luft- und Raumfahrt

Disc Pasture Meter

ECMWF in HAMburg

European Center for Medium Range Weather Forecasts

Eddy-Kovarianz

Environmental Policy Integrated Climate

ECMWF Re-Analyse

Food and Agriculture Organisation 
FC

FLUXNET

fPAR

GCOS

GDD

GEP

GIS

GL

GLC2000

GloPEM

GPP

GTOS

HA

HWSD

IIASA

ILW

INW

IPCC

JRC

KP

LAI

LCCS

LPJ

LUE

MODIS
Fieldcapacity

FLUX tower NETwork

fraction of absorbed Photosynthetically Active Radiation

Global Climate Observing System

Growing Degree Days

Gross Ecosystem Exchange

Geoinformationssystem

Grasland

Global Land Cover für das Jahr 2000

Global Production Efficiency Model

Gross-Primary-Production

Global Terrestrial Observing System

Harmonische Analyse

Harmonized World Soil Database

International Institute for Applied Systems Analysis

Immergrüner Laubwald

Immergrüner Nadelwald

Intergovernmental Panel on Climate Change

Joint Research Center

Kulturpflanzen

Leaf Area Index

Land Cover Classification System

Lund-Potsdam-Jena

Light Use Efficiency

MODerate-resolution Imaging Spectrometer 
MW

NADPH

NASA

NDVI

NEE

NLC

NOAA

NPP

NRMSE

NTSG

ORCHIDEE

PAR

PDF

PK

POSTEL

RMSE

RuBisCO

SK

SPOT

SRTM

SVAT

TOMS

UNCCD

UNEP

UNFCCC
Mischwald

Nicotinamid-Adenin-Dinukleotid-Phosphat

National Aeronautics and Space Administration

Normalised Difference Vegetation Index

Net Ecosystem Exchange

National Land Cover

National Oceanic and Atmospheric Administration

Netto-Primär-Produktion

Normalized Root Mean Square Error

Numerical Terradynamic Simulation Group

ORganizing Carbon and Hydrology In Dynamic EcosystEms

Photosynthetic Active Radiation

Probability Density Function

Primärklasse

Pôle d'observation des surfaces continentales par télédétection

Root Mean Square Error

Ribulose-1,2-bisphosphat-carboxylase/-oxygenase

Sekundärklasse

Satellite Pour l'Observation de la Terre

Shuttle Radar Topography Mission

Soil Vegetation Atmosphere Transfer

Total Ozone Mapping Spectrometer

United Nations Convention to Combat Desertification

United Nations Environment Programme

United Nations Framework Convention on Climate Change 
VALERI

VEGA2000

VITO

VPD

WDC-RSAT

WGS84
Validation of LAnd European Remote sensing Instruments

VEGETATION data for Global Assessment in 2000

Vision on Technology

Vapour Pressure Deficit

World Data Center for Remote Sensing of the Atmosphere

World Geodetic System 1984 


\section{$1 \quad$ Einleitung}

Klimamodelle leisten inzwischen seit mehreren Jahrzehnten einen bedeutenden Beitrag zum Verständnis der dynamischen Vorgänge in der Geophysik und speziell in der Atmosphärenphysik unseres Planeten. Die Beschreibung der globalen Wechselwirkungen kann als Werkzeug verstanden werden, großskalige Zusammenhänge in Bezug zueinander zu setzen und diese besser verständlich zu machen. Die Relevanz der globalen Auswirkungen durch regionale Eingriffe in das Ökosystem, wie beispielsweise die Abholzung von Regenwaldgebieten in Südamerika oder natürliche Ereignisse wie Vulkanausbrüche mit intensivem Ascheausstoß in die Atmosphäre, werden aktuell mehr diskutiert denn je (IPCC, 2001; GCOS, 2003; IPCC, 2007a,b). Entsprechend groß ist das Interesse, Aussagen über zukünftige Entwicklungen $\mathrm{zu}$ erhalten, wenn beispielsweise aktuelle Trends des $\mathrm{CO}_{2}$ Ausstoßes und die dadurch bedingte Temperaturänderung anhalten. Ebenso werden unterschiedliche Szenarien bezüglich der Entwicklung der Weltbevölkerung, der Nutzung verschiedener Energiequellen oder der weltwirtschaftlichen Entwicklung auf deren Auswirkungen auf das globale Klima geprüft (Nakicenovic, 2000). Diese sollen einerseits verdeutlichen, dass bezüglich des Umgangs mit der Umwelt und deren Ressourcen durch den Menschen dringender Handlungsbedarf besteht. Andererseits sollen mit Zukunftsmodellen positive Handlungsweisen gefördert und entsprechende Empfehlungen für Entscheidungsträger und Einzelpersonen mit Modellergebnissen untermauert werden.

In den frühen 1960er Jahren entstanden die ersten Klimamodelle zur Bestimmung des Einflusses von Treibhausgasen (Wasserdampf, Kohlendioxid und Ozon) auf die thermischen Eigenschaften der Atmosphäre, die sich aus den frühen Modellen zur Wettervorhersage weiterentwickelten (Manabe und Möller, 1961; Manabe und Strickler, 1964). Diese eindimensionalen Modelle gehören zu den Strahlungs-Konvektions-Modellen, bei denen einerseits der Strahlungstransport durch die atmosphärischen Schichten und andererseits der Wärmetransport durch Konvektion berücksichtigt werden. Darauf folgten die ersten Energiebilanzmodelle (Budyko, 1969; Sellers, 1969), die sich mit den Strahlungsflüssen und den turbulenten Flüssen von fühlbarer und latenter Wärme beschäftigen. Horizontale Wechselwirkungen wurden in diesen Modellen zunächst nicht berücksichtigt. Dies konnte erst in den späten 1970er Jahren mit den sogenannten statistisch-dynamischen Modellen erreicht werden (Kurihara, 1970; Vernekar und Duck Chang, 1978; Saltzman, 1978). Größtes Hemmnis bei der weiteren Entwicklung immer komplizierterer Modelle war damals wie heute die Verfügbarkeit an Rechenleistung, um die immer aufwändiger werdenden Berechnungen durchführen zu können. Daher wurden immer wieder Vereinfachungen der physikalischen Gegebenheiten gesucht und entwickelt, die es ermöglichten, großskalige, dreidimensionale Simulationen der Atmosphäre in grober Auflösung mit globaler Abde- 
ckung durchzuführen. Darin wurden insbesondere die kleinskaligen Prozesse wie turbulente Strömungen und Konvektionen als Parametrisierungen dargestellt. Durch die exponentielle Entwicklung der Rechenleistung gegen Ende des 20. Jahrhunderts konnten sich unterschiedlich spezialisierte Forschungsrichtungen innerhalb der Klimamodellierung etablieren. Ein Überblick über die Entwicklung der Klimamodellierung seit den 1960er Jahren findet sich in McGuffie und Henderson-Sellers (2001).

Die Bandbreite der aktuellen Modelle erstreckt sich von spezialisierten, lokalen Beschreibungen einfacher Ökosysteme mit einfacher Parametrisierung über regionale und kontinentale Ansätze, die lokale Gegebenheiten grobskaliger darstellen und dabei als Randbedingung auf allgemeine Annahmen globaler Einwirkungen zurückgreifen, bis hin zu den globalen Klimamodellen, die auf vergleichsweise grober Auflösung ( $a b 0.5^{\circ}$ ) die komplexe Dynamik der Atmosphäre zu beschreiben versuchen. Der stetige Anstieg der Kohlendioxidkonzentration in der Atmosphäre innerhalb der letzten 100 Jahre (Keeling, 1960; Etheridge et al. 1996; Sundquist und Keeling, 2009) führte zu einer intensiven Ausweitung der Erforschung des globalen wie auch regionalen Kohlenstoffkreislaufes innerhalb der Klimamodelle sowie durch eigenständige Modelle. Anwendungsgebiet dieser Modelle ist einerseits die weitere Entwicklung der Erderwärmung aufgrund der Erhöhung der Treibhausgaskonzentrationen und andererseits deren Auswirkungen auf unsere Umwelt. In globalen Klimamodellen werden daher wichtige physikalische Vorgänge in der Erdatmosphäre, den Ozeanen und auf der Erdoberfläche und schließlich die Wechselwirkungen zwischen den Systemen beschrieben. Meist werden dabei einzelne Prozesse stark vereinfacht parametrisiert oder gar als konstante Größen repräsentiert. Darunter zählen in vielen Modellen auch die Vorgänge in der Biosphäre und deren Wechselwirkung mit der Atmosphäre (Foley et al., 1998; Smith et al., 2001).

Die Biosphäre stellt mit ca. $800 \mathrm{GtC}$ nur etwa $0.001 \%$ des globalen Kohlenstoffaufkommens ( $\left.7510^{6} \mathrm{GtC}\right)$ dar und ist zusammen mit der Atmosphäre (765 GtC) einer der kleineren Kohlenstoffspeicher der Erde. Im Vergleich dazu befinden sich ca. $38200 \mathrm{GtC}$ in den Ozeanen bzw. etwa 99.8 \% des globalen Kohlenstoffs in Gestein (Sabine et al., 2004; Sarmiento and Gruber, 2006; IPCC, 2007a,b). Allerdings sind zwischen den beiden Systemen (Biosphäre und Atmosphäre) die höchsten Austauschraten innerhalb vergleichsweise kurzer Zeiträume zu finden, was die Vorgänge besonders interessant für die Modellbetrachtung macht (Schimel, 1995). Die Flussraten von Landpflanzen durch die Photosynthese betragen beispielsweise etwa $120 \mathrm{GtC}$ pro Jahr, denen etwa $119 \mathrm{GtC}$ pro Jahr durch die Pflanzenatmung gegenüberstehen (IPCC, 2007a,b). Doch auch diese Werte sind zeitlich nicht konstant und können von Jahr zu Jahr stark schwanken, insbesondere durch Eingriffe in das Ökosystem durch Abholzung oder Aufforstung, Umwandlung von Gras- in Acker- 
land, aber auch die Auswirkungen des Klimawandels (Dufresne et al., 2002; Myhre and Myhre, 2003; Jiao and Zhao, 2009; Qi et al., 2012).

Die Relevanz der vegetativen Produktivität (d.h. der Aufnahme von $\mathrm{CO}_{2}$ aus der Atmosphäre und Verarbeitung zu Biomasse) wurde schließlich als primäre Variable zur Beobachtung der ökologischen Funktionalität und voranschreitender Degradationsprozesse definiert (CGER 2000; Metz et al., 2007; GTOS, 2009). Daher ist es sinnvoll, dies auch in den Modellen durch komplexere Beschreibungen der Wechselwirkung zwischen Biosphäre und Atmosphäre in eigenständigen Modulen zu berücksichtigen. Dabei werden meist zwei unabhängige Ansätze verfolgt. Zum einen die Ableitung der Produktivität der Pflanzen nach dem Ansatz von Monteith (1965) und Monsi und Saeki (1953) als lineare Funktion von photosynthetisch aktiver Strahlung und Lichtnutzungseffizienz. Beispiele für solche Modelle sind EPIC (Williams et al., 1984), CASA (Potter et al., 1993) oder C-Fix (Veroustraete et al., 1996).

Zum anderen der Ansatz zur physikalischen Beschreibung der photosynthetischen Reaktionen nach Farquhar et al. (1980). Die photosynthetischen Reaktionen werden durch physikalische Formulierungen in Abhängigkeit meteorologischer und pflanzenphysiologischer Parameter nachvollzogen. Für eine komplette Beschreibung des Kohlenstoffkreislaufes der Landvegetation ist es notwendig auch die Wechselwirkung mit dem Boden zu berücksichtigen. Modelle, die dies mit einschließen, werden als Soil-Vegetation-Atmosphere Transfer (SVAT) Modelle bezeichnet. Dieser Ansatz wird unter anderem in den Modellen LPJ (Prentice et al., 1992), BIOME3 (Haxeltine und Prentice, 1996) und ORCHIDEE (Krinner et al., 2005) verwendet. Als Resultat der Berechnungen geht der Austausch von Kohlenstoff zwischen Vegetation und Atmosphäre in übergeordnete Klimamodelle ein. Inzwischen werden die SVAT-Modelle allerdings auch als eigenständige Modelle für konkrete Fragestellungen auf Ebene der Biosphäre selbst verwendet. Abgekoppelt von den globalen Anforderungen der Klimamodelle und der damit verbundenen Rechenaufwendigkeit, können höhere räumliche und zeitliche Auflösungen erzielt werden, um regionale Problemstellungen anzugehen. Diese beziehen sich speziell auf die Dynamik des Kohlenstoffkreislaufes der Vegetation. Sie beinhalten unter anderem Untersuchungen der Biodiversität, der Berechnung von Bioenergiepotentialen sowie der Produktivität unterschiedlicher Vegetationstypen oder Strategien des nachhaltigen Landnutzungsmanagements.

Ein wesentlicher Punkt zur Beobachtung anfälliger Ökosysteme ist die zeitliche Entwicklung der Produktivität und des Zustandes der Vegetation. Speziell das Phänomen der Landdegradation und der Wüstenausbreitung in Trockengebieten beeinflusst beispielsweise die Anbau- und Haltungsbedingungen von Bauern und Viehhirten und beeinträchtigt dadurch 
die Versorgungssicherheit mit Lebensmitteln. Dabei werden beide Prozesse hauptsächlich durch den Menschen selbst verursacht, wie z. B. durch zu intensive Landnutzung, Weidewirtschaft oder Waldrodung (Evans und Geerken, 2004; Wessels et al., 2007; Al-Awadhi, 2011). Aber auch klimatische Ereignisse wie lange Dürreperioden oder durch Wind und Wasser verursachte Bodenerosion tragen $\mathrm{zu}$ dem Prozess der Landdegradation bei, da Trockengebiete als sensible Ökosysteme bereits sehr anfällig gegenüber kurzfristigen Störungen sind. Daher ist es langfristig sehr wichtig, den Zustand solcher Gebiete zu beobachten und ständig zu bewerten, welche Auswirkungen diese Störungen haben.

Die Bewertung der Landdegradation und der Entwicklung der Pflanzenproduktivität in den Trockengebieten des südlichen Afrika ist bereits seit längerer Zeit Bestandteil wissenschaftlicher Studien (Abel und Blaikie, 1989; Ringrose et al., 1999; Wessels et al., 2004, 2008; Hoffman und Todd, 2000). Satellitenmessungen spielen dabei eine immer wichtiger werdende Rolle, vor allem bei der Beobachtung und der Bewertung des Zustandes von Trockengebieten. Sie ermöglichen die Erkennung von Anzeichen der Wüstenbildung. Nur durch die Erdbeobachtung mittels Satelliten ist es möglich kontinuierliche und regelmäßige Daten über relevante Parameter zu erhalten, die zur großflächigen Analyse von Trockengebieten eingesetzt werden können. Durch die Verwendung von Erdbeobachtungsdaten in Verbindung mit meteorologischen Zeitreihen als antreibende Parameter eines Vegetationsmodells können sowohl die klimatischen als auch die anthropogenen Einflüsse auf das Ökosystem untersucht werden. Dies geschieht in der vorliegenden Arbeit mittels des Vegetationsmodells BETHY/DLR (Biosphere Energy Transfer HYdrology - erweitert am Deutschen Zentrum für Luft- und Raumfahrt), das durch Klimadaten angetrieben wird und zusätzliche Informationen aus der Fernerkundung zur Phänologie und zur Vegetationsverteilung erhält (Knorr, 1997; Knorr und Heimann, 2001a,b; Wisskirchen, 2005). BETHY/DLR ist ein SVAT-Modell nach dem Ansatz von Farquhar et al. (1980). Dieser ermöglicht es die photosynthetische Aktivität der Vegetation zu berechnen und die Kohlenstoffbilanz zwischen Atmosphäre und Biosphäre zu bestimmen. Als Ergebnis wird die Menge an Kohlenstoff bestimmt, die über einen bestimmten Zeitraum von der betrachteten Vegetation als Biomasse aufgenommen wurde.

In der vorliegenden Arbeit sollen Zeitreihen der Produktivität über 12 Jahre verwendet werden, um die Dynamik der Vegetationsentwicklung in den regenarmen Trockengebieten des südlichen Afrika südlich von $15^{\circ}$ Süd zu bewerten. Ein Vergleich mit der Landnutzung und den entsprechenden klimatischen Zeitreihen ermöglicht die Unterscheidung zwischen klimabedingter und durch den Menschen verursachter Beeinträchtigung der Pflanzenentwicklung. Eine genaue Beschreibung des Arbeitsgebietes sowie des verwendeten Modells liefert zunächst Kapitel 2. Da das Modell in dieser Arbeit erstmalig für Trockengebiete 
angewandt wird, ist in der Vorbereitung eine Anpassung an die speziellen klimatischen Gegebenheiten notwendig. Dies beinhaltet zum einen die Vorprozessierung der Eingangsdaten, zum anderen die Regionalisierung einzelner Modellparameter und Ergänzungen am Modell selbst. Hinzu kommt die Validierung der klimatischen Eingangsdatensätze für das Arbeitsgebiet. Ein weiterer Teil dieser Arbeit ist die Implementierung eines Phänologiemodells in BETHY/DLR, zur Vorbereitung für die Szenarienmodellierung. Dies ermöglicht künftig den Vegetationsindex, als notwendigen Eingangsparameter, aus den klimatischen Werten einzelner Szenarien zu bestimmen. Die Beschreibung dieses Modells schließt sich direkt an die allgemeine Darstellung des Vegetationsmodells BETHY/DLR an. In Kapitel 3 wird die Sensitivität des Vegetationsmodells auf Veränderungen in den Eingangsparametern analysiert. Die Modellergebnisse werden in Kapitel 4 beschrieben und unter Verwendung von Messdaten und Modellvergleichen bewertet. Kapitel 5 behandelt die Indexierung der Landdegradation aufgrund von Zeitreihen der Modellergebnisse und der meteorologischen Parameter. Die Arbeit schließt in Kapitel 6 mit der Zusammenfassung der Ergebnisse. 



\section{$2 \quad$ Methodenbeschreibung}

\subsection{Landdegradation in Trockengebieten}

Die Bewertung des Prozesses der Landdegradation bzw. der Desertifikation erfordert zunächst eine objektive Definition der beiden Begriffe selbst. In der Konvention der Vereinten Nationen zur Bekämpfung der Desertifikation (United Nations Convention to Combat Desertification, UNCCD) von 1994 wurde Landdegradation definiert als „die Verringerung oder der Verlust der biologischen oder wirtschaftlichen Produktivität und der Vielseitigkeit von natürlich oder künstlich bewässerten Anbauflächen oder von Wiesen und Weideland, forstwirtschaftlich genutzten Flächen und Wäldern in ariden, semiariden und trockenen subhumiden Gebieten infolge der Nutzung des Landes oder infolge eines einzelnen oder mehrerer miteinander verknüpfter Prozesse einschließ1ich solcher, die sich aus menschlichen Tätigkeiten und Siedlungsmustern ergeben, wie:

(i) durch Wind und/oder Wasser verursachter Bodenerosion,

(ii) die Verschlechterung der physikalischen, chemischen und biologischen oder wirtschaftlichen Eigenschaften des Bodens,

(iii) das Verschwinden des natürlichen Pflanzenbestandes auf lange Sicht“ (UNCCD, 1994).

Der Begriff der Desertifikation wird darüber hinaus definiert als ,die Landverödung [Landdegradation] in ariden, semiariden und trockenen subhumiden Gebieten infolge verschiedener Faktoren, einschließlich Klimaschwankungen und menschlicher Tätigkeiten“ (UNCCD, 1994). Prinzipiell korreliert die Landdegradation mit einem Verlust an biologischer und ökologischer Produktivität durch Bodenerosion, Versalzung, Krustenbildung oder dem Verlust an Fruchtbarkeit des Bodens. Dadurch wird der Bewuchs, speziell dessen Biodiversität und dessen Dichte, stark beeinflusst (LeHouérou, 1996). Die Kohlenstoffreservoirs der Landoberfläche im Boden und gespeichert als Biomasse stellen empfindliche Indikatoren der Degradation und dem Wandel in Klima und Umweltbedingungen dar. Daher wurde die Landbiomasse als eine der essentiellen Klimavariablen mit hohem Einfluss auf die Anforderungen der UNFCCC (United Nations Framework Convention on Climate Change) definiert (GCOS, 2005). Änderungen in diesem Reservoir bedingen eine kritische Rückkopplung auf das Klima und die Treibhausgasbilanz (GTOS, 2009). Speziell die Nettoprimärproduktion, also der Kohlenstoffaustausch zwischen Atmosphäre und Vegetation wurde als primäre Variable identifiziert, um die ökologische Funktionalität und andauernde Degradationsprozesse zu beobachten (CGER, 2000).

Weltweit sind etwa $70 \%$ aller Trockengebiete von Landdegradation betroffen (UNCCD, 2008), was einer Gesamtfläche von 3.6 Millionen Hektar entspricht. Gerade diese Gebiete sind anfällig für den Verlust ihrer Produktivität, da sie ohnehin starken Schwankungen des 
Jahresniederschlages ausgesetzt sind. Das sensible Ökosystem ist zwar an diese widrigen Bedingungen angepasst, kann aber bereits durch geringe Störungen aus dem Gleichgewicht geraten. Hinzu kommt, dass es sich hier meist um Gebiete mit fehlender oder schlecht entwickelter Infrastruktur handelt, deren Bevölkerung erschwerten Zugang zu Informationen über eine nachhaltige Bewirtschaftung hat.

Im Vergleich zu feuchten oder moderaten Klimaten ist die Beschreibung des Kohlenstoffaustausches zwischen Ökosystem und Atmosphäre in semiariden und vollariden Gebieten wesentlich komplexer. Dies folgt aus der höheren Sensibilität und der stärkeren Rückkopplung der Ökosysteme auf variable Umwelteinflüsse. Die Landdegradation in ariden oder semiariden Gebieten unterliegt verschiedenen Faktoren, von biophysikalischen bis sozioökonomischen (Hoffman und Todd, 2000). Die Degradation des Bodens wird hauptsächlich durch Wind- und Wassererosion verursacht, zusätzlich aber auch durch Überdüngung oder Versalzung verstärkt. Die Degradation der Vegetation vollzieht sich dagegen über den Verlust an Fläche, veränderte Artenzusammensetzung, das Einführen fremder Pflanzenarten, Überweidung oder Abholzung. Außerdem sind speziell in den ländlichen Regionen und den Savannengebieten des südlichen Afrikas die Biome stark geprägt durch niedrige und unregelmäßige Niederschlagsereignisse. Zusätzlich trägt die intensive Weidelandnutzung zu der starken Überbeanspruchung der regionalen Vegetation bei (Perkins und Thomas, 1993; Dougill et al., 1999). Dies führt in den anfälligen Trockengebieten zu starkem Vegetationsrückgang bis hin zur Ausweitung von Wüstengebieten. Damit einher geht entsprechend der Verlust an Kohlenstoffspeichern, sowohl in Form der Vegetation als auch im Boden. 


\subsection{Das Untersuchungsgebiet}

Die Bewertung der Landdegradation im südlichen Afrika war bereits Bestandteil verschiedenster wissenschaftlicher Untersuchungen. Abel und Blaikie (1989) analysierten unterschiedliche Erhaltungsstrategien in kommunalen Weidegebieten in Botswana und Simbabwe. Dabei wurden die jeweiligen landwirtschaftlichen Praktiken und deren Einfluss auf die Trockengebiete berücksichtigt. Das betrachtete Untersuchungsgebiet erstreckt sich dabei über ca. $700 \mathrm{~km}^{2}$. Mittels Karten aus Fernerkundungsdaten konnten Ringrose et al. (1999) in etwa $1600 \mathrm{~km}^{2}$ Weideland der südlichen Botswana-Kalahari verschiedene Merkmale wie blanker Boden, erodierte Abschnitte und Vegetation mit unterschiedlichem Bedeckungsgrad identifizieren. Auf Basis von Haushaltsbefragungen und Umfragen durch Stringer und Reed (2007) bei Landwirten in Distrikten Botswanas und in Swasiland zum Zustand und der Entwicklung der Vegetation ihres Landgutes, konnte für diese Gebiete eine genaue Bestandsaufnahme zum Zeitpunkt der Umfragen erstellt werden. Durch Hoffman und Todd (2000) konnte bereits zuvor durch Umfragen in den 367 Regierungsbezirken Südafrikas eine aussagekräftige Karte des Degradationszustandes für die Wachstumsperiode 1997/98 erstellt werden. Dabei stellten sich die Regionen KwaZulu-Natal, Limpopo und Nord- und Ostkap als am stärksten betroffen heraus.

Ein erster Ansatz die durch den Menschen beeinflusste Landdegradation mittels Fernerkundung langfristig zu erfassen wurde durch Wessels et al. (2004, 2008) verfolgt. Die Autoren verwendeten NDVI (Normalised Difference Vegetation Index) Zeitreihen für den Zeitraum 1985-2003 mit 1km Auflösung des AVHRR (Advanced Very High Resolution Radiometer) Sensors, der auf Satelliten der National Oceanic and Atmospheric Administration (NOAA) betrieben wird. Aus der Kombination von Jahressummen des NDVI mit den entsprechenden Niederschlagsmengen konnte im nördlichen Südafrika unterschieden werden zwischen Landdegradation verursacht durch den Menschen und aufgrund von Dürreereignissen. Die meisten dieser Aktivitäten wurden auf regionaler Ebene für einzelne, spezielle Biome durchgeführt. Jeder dieser Ansätze lässt entweder die zeitliche oder die räumliche Abdeckung vermissen. Entweder ist der Ansatz nur auf regionale bzw. lokale Untersuchungsgebiete beschränkt und schwer auf größere Bereiche übertragbar oder er berücksichtigt nur einen kurzen Zeitraum, wenn nicht sogar nur eine einzelne Momentaufnahme. Daher fehlt es nach wie vor an nationalen bis subkontinentalen Ansätzen, speziell auch in der hier betrachteten Region des südlichen Afrikas.

Durch moderne Vegetationsmodelle, angetrieben durch Zeitserien von Erdbeobachtungsdaten, können mit höherer Auflösung $\left(<0.25^{\circ}\right)$ große Gebiete abgedeckt und über längere Zeit beobachtet werden. Anstatt NDVI Summen zu verwenden, besteht die Möglichkeit zusätzliche klimatische Faktoren zu berücksichtigen, die die Produktivität der Vegetation 
beeinträchtigen können. Zwar gilt der NDVI als Indikator der Produktivität der Pflanzen auf großskaliger Ebene, kleinskalige Einflüsse werden dadurch allerdings nicht mit berücksichtigt.

Eine detailliertere Betrachtung der Umwelteinflüsse auf die photosynthetischen Reaktionen der Pflanzen ist mit den eingangs beschriebenen SVAT Modellen möglich. Dabei wird auf Blattebene die Austauschrate an Kohlenstoff zwischen der Atmosphäre und der Vegetation unter Berücksichtigung des Energie- und Wasserhaushaltes bestimmt. Ein solches Modell stellt das von Knorr (1997) entwickelte Biosphere Energy Transfer Hydrology Model (BETHY/DLR) dar, das am Deutschen Fernerkundungsdatenzentrum (DFD) im Deutschen Zentrum für Luft- und Raumfahrt (DLR) betrieben wird (Knorr, 1997; Knorr und Heimann, 2001a, 2001b; Wisskirchen, 2005). Aus meteorologischen und fernerkundungsbasierten Datenreihen wird die Nettoprimärproduktion (NPP) in täglichen Zeitschritten berechnet (eine nähere Beschreibung folgt in Kapitel 2.3). Eine Analyse längerer Zeitreihen der NPP in ariden und semi-ariden Gebieten kann Informationen über den Prozess der Landdegradation liefern. Eine zusätzliche Analyse der klimatischen Eingangsdaten kann bei der Unterscheidung zwischen klimabedingten und von Menschen verursachten Einflüssen in diesem Prozess unterstützen (Kapitel 2.5).

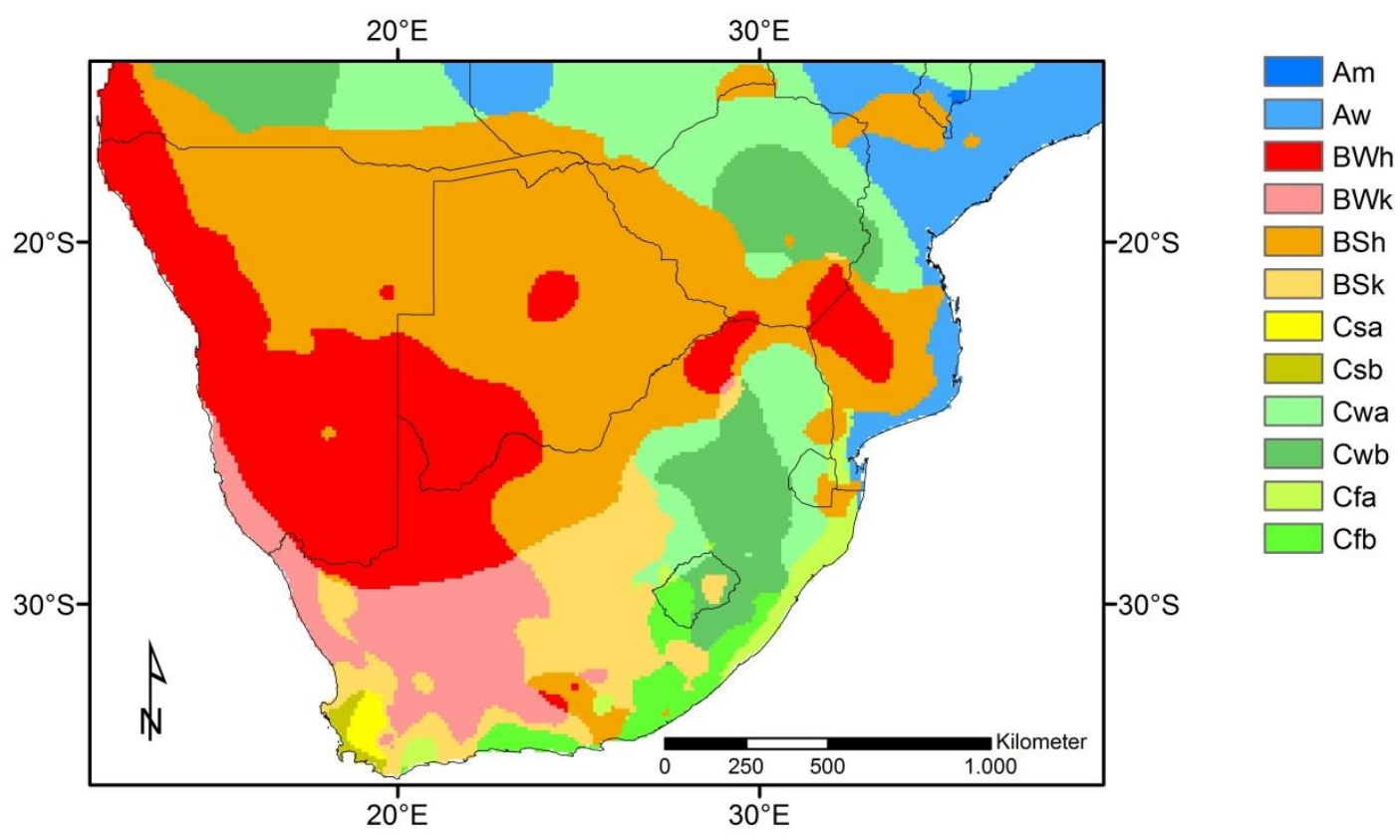

Abbildung 2.1: Köppen-Geiger Klassifikation der Klimatypen im Arbeitsgebiet des südlichen Afrika. Die Beschreibung der Legende erfolgt in Tabelle 2.1 und Tabelle 2.2.

Als Untersuchungsregion wurde das Gebiet des südlichen Afrikas gewählt. Dieses umfasst die Länder Namibia, Südafrika, Lesotho, Swasiland, Botswana und Zimbabwe, sowie Teile von Mosambik, Angola, Sambia und Malawi. Die geographische Ausdehnung erstreckt 
sich von $11.5^{\circ}$ Ost $15.0^{\circ}$ Süd als nordwestlichstem Punkt und $39.5^{\circ}$ Ost $35.0^{\circ}$ Süd als südöstlichstem Punkt (Abbildung 2.1).

Wie die Verteilung der Köppen-Geiger Klimaklassifikation (Kottek et al., 2006) zeigt, ist die gesamte westliche Hälfte des Gebietes durch aride Bedingungen geprägt (Typ B, Abbildung 2.1), teilweise in Form von Steppen- (BS) und Wüstengebieten (BW). In Botswana und dem südlichen Zimbabwe zieht sich das trockene und heiße Klima bis in den Osten und bestimmt noch weite Teile der südlichen Provinzen von Mosambik. Warm-gemäßigte Klimazonen (Typ C) sind in Südafrika an den südlichen und südöstlichen Küstenregionen zu finden sowie in den höher gelegenen Regionen im Osten des Landes. Auch oberhalb des 16ten Breitengrades ist gemäßigtes bis äquatoriales Klima vorzufinden. Die Einteilung in die unterschiedlichen Klimatypen erschließt sich aus den Eigenschaften bezüglich mittlerer Temperaturen und aufsummierter Niederschläge (Tabelle 2.1 und Tabelle 2.2).

Tabelle 2.1: Unterteilung der Hauptklimazonen (A: äquatorial, B: arid, C: warm-gemäßigt) in Klimatypen des Arbeitsgebietes unter Berücksichtigung der Niederschlagscharakteristika (m: monsunal, S: Savanne, W: Wüste, s: sommertrocken, w: wintertrocken und f: feuchtgemäßigt) nach Köppen und Geiger (Köppen, 1900; Geiger, 1954; Kottek et al., 2006).

\begin{tabular}{|c|c|c|}
\hline Klimatypen & Beschreibung & Kriterien \\
\hline \multirow[t]{2}{*}{ A } & Tropisches Regenwald- oder Savannenklima & \\
\hline & ohne Winter & $\mathrm{T}_{\min } \geq+18{ }^{\circ} \mathrm{C}$ \\
\hline Am & Monsun geprägt & $\mathrm{P}_{\mathrm{ann}} \geq 25\left(100-\mathrm{P}_{\min }\right)$ \\
\hline Aw & Savannenklima & $\mathrm{P}_{\min }<60 \mathrm{~mm}$ im Winter \\
\hline $\mathrm{B}$ & Trockenklima & $\mathrm{P}_{\mathrm{ann}}<10 \mathrm{P}_{\mathrm{th}}$ \\
\hline $\mathrm{BS}$ & Steppenklima & $\mathrm{P}_{\mathrm{ann}}>5 \mathrm{P}_{\text {th }}$ \\
\hline BW & Wüstenklima & $\mathrm{P}_{\mathrm{ann}} \leq 5 \mathrm{P}_{\mathrm{th}}$ \\
\hline $\mathrm{C}$ & Warm-gemäßigtes Klima & $-3{ }^{\circ} \mathrm{C}<\mathrm{T}_{\min }<+18^{\circ} \mathrm{C}$ \\
\hline \multirow[t]{2}{*}{ Cs } & warmes sommertrockenes Klima & $\mathrm{P}_{\mathrm{s}, \min }<\mathrm{P}_{\mathrm{w}, \min }, \mathrm{P}_{\mathrm{w}, \max }>3 \mathrm{P}_{\mathrm{s}, \text { min }}$ und \\
\hline & & $\mathrm{P}_{\mathrm{s}, \min }<40 \mathrm{~mm}$ \\
\hline $\mathrm{Cw}$ & warmes wintertrockenes Klima & $\mathrm{P}_{\mathrm{w}, \text { min }}<\mathrm{P}_{\mathrm{s}, \min }$ and $\mathrm{P}_{\mathrm{s}, \max }>10 \mathrm{P}_{\mathrm{w}, \text { min }}$ \\
\hline $\mathrm{Cf}$ & feucht-gemäßigtes Klima & weder Cs noch $\mathrm{Cw}$ \\
\hline
\end{tabular}

Tabelle 2.2: Unterteilung der Temperaturklassen im Arbeitsgebiet nach Köppen und Geiger (Köppen, 1900; Geiger, 1954; Kottek et al., 2006).

\begin{tabular}{lll}
\hline Temperaturklassen & Beschreibung & Kriterien \\
\hline $\mathrm{a}$ & heiße Sommer & Mitteltemperatur des wärmsten Monats über $+22^{\circ} \mathrm{C}$ \\
$\mathrm{b}$ & warme Sommer & Mitteltemperatur des wärmsten Monats unter $+22^{\circ} \mathrm{C}$, mindestens \\
& & vier Monate mit Mitteltemperaturen von wenigstens $+10^{\circ} \mathrm{C}$ \\
$\mathrm{h}$ & heiß & Jahresmitteltemperatur über $+18^{\circ} \mathrm{C}$ \\
$\mathrm{k}$ & kalt & Jahresmitteltemperatur unter $+18^{\circ} \mathrm{C}$ \\
\hline
\end{tabular}


Für die Betrachtung von Vegetationsveränderungen in Trockengebieten sind die Regionen mit Wüsten- (BW) und Steppenklima (BS) von Interesse, welche die größte Fläche des Arbeitsgebietes bestimmen. Die klimatischen Eigenschaften des südlichen Afrika sind anhand von Langzeitmittelwerten der Datenreihen des ECMWF (European Center for Medium Range Weather Forecasts) berechnet aus dem Zeitraum 1989 bis 2010 in Abbildung 2.2 zu sehen. Die Verteilung des jährlichen Niederschlags (Abbildung 2.2a) spiegelt in Verbindung mit den mittleren Temperaturen (Abbildung 2.2c) die Klassifikationen des KöppenGeiger Schemas wieder.

(a)

(c)
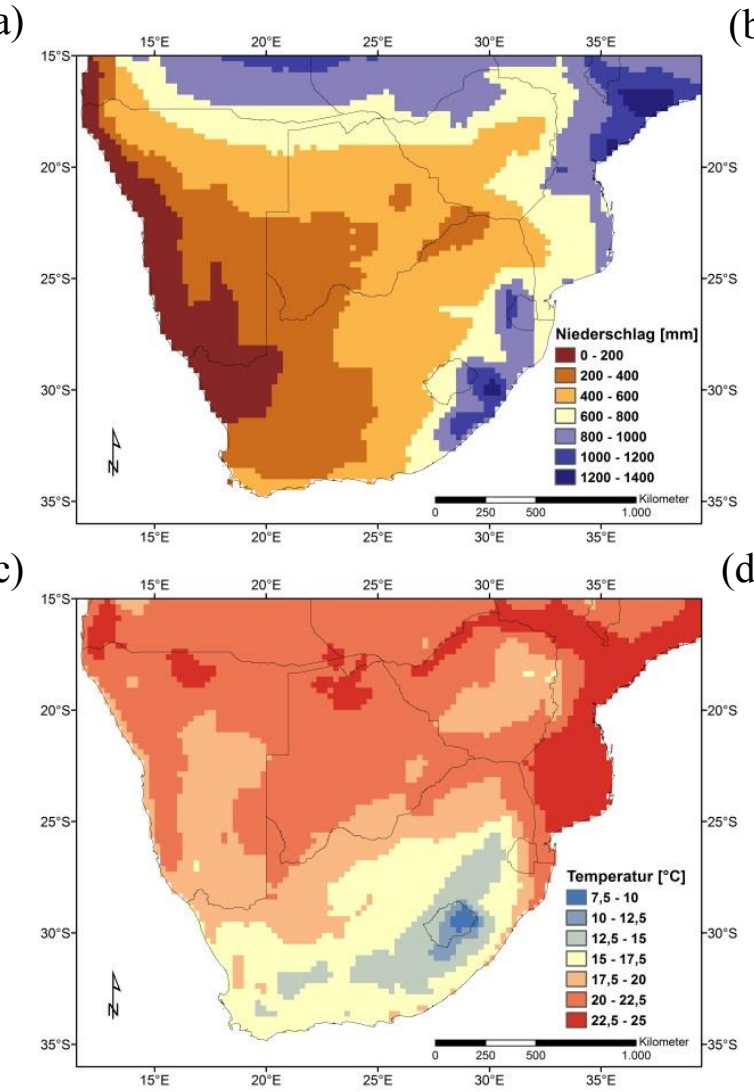

(b)

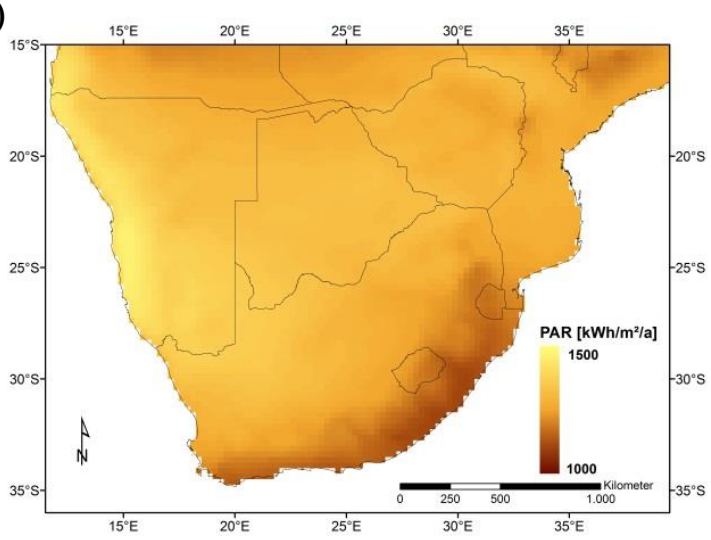

d)

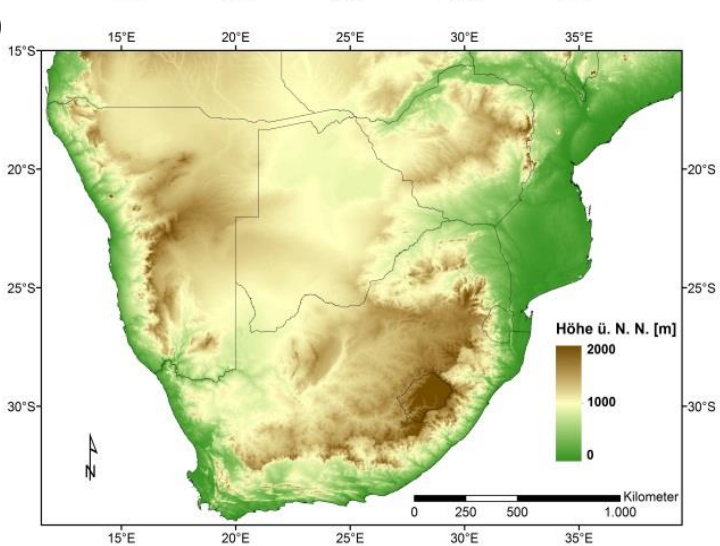

Abbildung 2.2: Klimatische Eigenschaften des Arbeitsgebietes berechnet aus Zeitreihen von ECMWF Parametern gemittelt über die jährlichen Werte des Zeitraumes 1989 bis 2010. Jahressumme des Niederschlags (a), Jahreseintrag an photosynthetisch aktiver Strahlung (PAR) (b), Jahresmittelwert der Temperatur (c) und Höhenprofil (d).

Niedrige bis sehr niedrige Niederschlagsmengen von unter $400 \mathrm{~mm}$ im Jahr kennzeichnen den Westen des Gebietes mit der Wüstenregion der Namib und der Kalahari als Extrema ( $<$ $200 \mathrm{~mm}$ ), nach Osten hin nimmt die Jahressumme bis zu Werten über $1000 \mathrm{~mm}$ im Jahr zu. Die Verteilung korreliert mit der photosynthetisch aktiven Strahlung (engl.: Photosynthetic Active Radiation, PAR) (Abbildung 2.2b), die entsprechend der zunehmenden Bewölkung von etwa $1500 \mathrm{kWh} \mathrm{m}^{-2} \mathrm{a}^{-1}$ in den Wüstenregionen auf $1000 \mathrm{kWh} \mathrm{m}^{-2} \mathrm{a}^{-1}$ an der Südost- 
küste abnimmt. Die Temperatur folgt einem positiven Trend von Süden nach Norden, wobei die niedrigsten Werte von unter $10{ }^{\circ} \mathrm{C}$ in den höher gelegenen Regionen von Lesotho und Südafrika zu finden sind. Das Höhenprofil des Arbeitsgebietes zeigt Abbildung 2.2d.

Anhand der Köppen-Geiger Klassifikation und den klimatischen Eigenschaften lassen sich bereits voraussichtliche Risikogebiete feststellen. Der Fokus in dieser Arbeit wird demnach auf den als Trockengebiete definierten Regionen (Typ B, Abbildung 2.1) liegen. Die Wüstenregionen der Namib und der Kalahari sind kaum bewachsen, daher wird dort auch keine Degradation der Vegetation feststellbar sein. Die Savannengebiete im Zentrum des Arbeitsgebietes (Ausläufer der Wüstengebiete, nordwestliches Südafrika, südliches Botswana) sind allerdings mit den geringen Niederschlagsraten und höheren Temperaturen besonders anfällig für sensible Reaktionen auf klimatische oder anthropogene Einflüsse. Letztere können bei sehr intensiver Landnutzung oder Beanspruchung allerdings auch in Gebieten, die nach den hier dargestellten klimatischen Gegebenheiten nicht als potentiell gefährdet eingestuft werden, zu einer Degradation führen. Daher können die Ergebnisse, die mit dem im folgenden Kapitel vorgestellten Modell erstellt werden, mit Hilfe der Zeitreihen der Klimatologie unterteilt werden in Gebiete, die aufgrund der klimatischen Gegebenheiten gefährdet sind und tatsächlich einen Rückgang der Produktivität erfahren und solche, die sich ohne klimatische Gefährdung, vermutlich aufgrund von menschlichen Einflüssen, im Prozess der Degradation befinden. 


\subsection{Das Vegetationsmodell BETHY/DLR}

Das Modell BETHY/DLR gehört zu den SVAT Modellen, welche unter Berücksichtigung der Wechselwirkungen zwischen dem Boden, der Pflanze und der Atmosphäre die Aufnahme von Kohlendioxid in der Pflanze bestimmen. Dabei werden Limitierungen durch Bodenwasser, Licht und Temperatur über die Berechnung der Wasser- und Energiebilanz des Systems betrachtet. Für diese Bilanzierung sind mehrere Eingangsparameter notwendig, die die Modellierung auf eine fundierte Datengrundlage in Form von Messwerten stellen. Die einzelnen Eingangsgrößen werden im Laufe der folgenden Modellbeschreibung benannt und im Anschluss näher beschrieben. Die Modellbeschreibung folgt im Wesentlichen den Ausführungen von Knorr (1997) und Wisskirchen (2005).

Bei dem Modell handelt es sich um die physikalische Beschreibung des biochemischen Prozesses der photosynthetischen Reaktion. Dabei wird auf die Beschreibung von Farquhar et al. (1980) zurückgegriffen, die die pflanzlichen Vorgänge durch verschiedene Widerstandsmodelle bzw. Leitfähigkeiten beschreibt. Darunter vor allem die stomatäre Leitfähigkeit, die den Öffnungsgrad der Stomata (Blattöffnungen an der Blattunterseite, über die der gesamte Gasaustausch stattfindet) in Abhängigkeit äußerer Faktoren, wie Temperatur, solare Einstrahlung oder Wasserverfügbarkeit steuert. Diese bestimmt im Wesentlichen die Aufnahmerate an Kohlendioxid aus der Atomsphäre und damit auch die mögliche Umsatzrate während der Photosynthese (Knorr, 1997).

\subsubsection{Die grundlegenden Funktionen}

Die wesentlichen Bestandteile des Modells sind die Beschreibung von Energie-, Strahlungs- und Wasserhaushalt. Die Energiebilanz an der Erdoberfläche wird beschrieben über die Nettostrahlung $R_{n}$, die über den Bodenwärmestrom $B$, den fühlbaren Wärmestrom zwischen Oberfläche und Umgebungsluft $H$ und dem latenten Wärmestrom des Systems $L$ verbraucht wird (Jones, 1983).

$$
R_{n}=B+H+L
$$

Der latente Wärmestrom $L$ berechnet sich aus der Evapotranspirationsrate $E$ der latenten Verdunstungswärme von Wasser $\lambda\left(2450 \mathrm{~kJ} \mathrm{~kg}^{-1}\right.$ bei $\left.20{ }^{\circ} \mathrm{C}\right)$ und der Dichte von Wasser $\rho_{W}\left(1000 \mathrm{~kg} \mathrm{~m}^{-3}\right)$ :

$$
L=\lambda \rho_{W} E
$$


Die Evapotranspiration geht zudem in die Berechnung der Wasserbilanz (Speicheränderung des Wasserreservoirs $\Delta W$ ) neben dem Wasserablauf $A$ als negativer Bestandteil ein. Der Eintrag wird über den Niederschlag $N$ beschrieben. Die verbleibende Wassermenge W wird in den Reservoirs im Boden $W_{s}$, auf der Vegetation $W_{i}$ oder als Schnee $W_{s n}$ gespeichert.

$$
N-E-A=\Delta W
$$

Der Wassereintrag kann in Form von Regen $N_{r}$ und Schneeschmelze $N_{s}$ erfolgen, wobei der Regen den Boden direkt erreichen kann $\left(N_{b}\right)$ oder von der Vegetation aufgefangen werden kann $\left(N_{i}\right)$. Auch die Evapotranspiration lässt sich entsprechend differenzieren:

$$
E=E_{b}+E_{s}+E_{i}+E_{t}
$$

$E_{b}$ ist die Verdunstung von Bodenwasser, $E_{s}$ die Sublimation von Schnee, $E_{i}$ die Verdunstung des Oberflächenreservoirs und $E_{t}$ die Transpiration der Vegetation (Knorr, 1997). $E_{t}$ beschreibt den Gasaustausch der Pflanze mit der Umgebungsluft. Dabei wird Kohlendioxid für die Photosyntheseprozesse von der Pflanze über Blattöffnungen, die Stomata, aufgenommen, wobei allerdings durch die Diffusion von Wasserdampf auch Flüssigkeit verloren geht. Daher haben die meisten Pflanzen die Möglichkeit, diese Leitfähigkeit des Gasflusses der Stomata über deren Öffnungsgrad zu regulieren. Die Beschreibung der Photosynthese hängt demnach stark mit der Beschreibung der Transpiration zusammen (Farquhar und Sharkey, 1982). Über die Energiebilanz sind die Oberflächentemperatur $T_{v}$ und damit die Evapotranspiration eng mit der Nettoeinstrahlung $R_{n}$ verbunden. Diese unterscheidet sich wesentlich bei Betrachtung von Boden und Vegetation. Daher werden diese Anteile getrennt $\left(R_{n v}\right.$ und $\left.R_{n b}\right)$ betrachtet:

$$
\begin{gathered}
R_{n v}=\left(1-t_{l, v}\right)\left(R_{L \downarrow}-R_{L \uparrow}-B\right)+a_{v} R_{s} \\
R_{n b}=t_{l, v}\left(R_{L \downarrow}-R_{L \uparrow}\right)+a_{s} R_{s}+\left(1-t_{l, v}\right) B
\end{gathered}
$$

$R_{L \downarrow}$ ist die langwellige thermische Strahlung von der Atmosphäre in Richtung der Oberfläche und $R_{L \uparrow}$ die Strahlung zurück von der Oberfläche (Knorr, 1997). Die Bestimmung des Bodenwärmestromes $B$ stützt sich auf die Annahmen von Verma et al. (1986) und Rosenberg (1974), die diesen als konstanten Faktor der Nettoeinstrahlung festlegen ( $\left.B=0.036 R_{n}\right) . R_{s}$ ist die gesamte solare Einstrahlung, die auch den photosynthetisch inak-

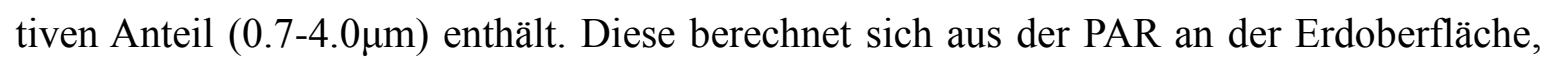
$R_{P A R} \cdot r_{P A R}$ (siehe Gleichungen (2.57) und (2.58)), und einem Konversionsfaktor $F$ (Pinker 
und Laszlo, 1992). $t_{l, v}$ ist die langwellige, thermische Transmissivität, $a_{v}=\left(1-\rho_{v}-a_{s, 0}\right) f_{P A R}$ ist das effektive Absorptionsvermögen kurzwelliger Strahlung der Vegetation und $a_{b}=\left(1-\rho_{b}\right)-\left(1-\rho_{b}-a_{b, 0}\right) f_{P A R}$ das des Bodens (mit $\rho_{v}=0.15$ der Albedo von dichter Vegetation (Brutsaert, 1982), $a_{b, 0}=0.05$ dem Anteil absorbierter PAR unter geschlossenem Blattbestand und $f_{P A R}$ dem Anteil an absorbierter PAR, Herleitung siehe Knorr, 1997). Die Albedo des Bodens $\rho_{b}$ berechnet sich entweder aus dem Bodenwassergehalt $W_{b}$ und Richtwerten der Albedo von trockenem bzw. feuchtem Boden (wobei zwischen drei Bodentypen unterschieden wird, siehe Tabelle 2.3) oder bei Schneebedeckung (Schneehöhe $h_{s} \neq 0$ ) aus der Albedo von Schnee $\rho_{s}$ abhängig vom Sonnenstand $\mu_{12}$ und der Wolkenbedeckung $n_{w}$ (Knorr, 1997).

$$
\rho_{b}=\left\{\begin{array}{ccc}
x \rho_{b, n}+(1-x) \rho_{b, t} & \text { wenn } & h_{s}=0 \\
\rho_{s} & \text { wenn } & \mu_{12} \leq 0 \\
\rho_{s}+\left(1-\rho_{s}\right) \rho_{s}^{3}\left(n_{w}^{2}+\left(1-1.3 n_{w}^{2}\right) e^{1-\left(1-\mu_{12}\right)^{-2}}\right) & \text { wenn } & \mu_{12}>0
\end{array}\right.
$$

Tabelle 2.3: Unterscheidung der Albedo für feuchten ( $\left.\rho_{b, n}\right)$ und trockenen $\left(\rho_{b, t}\right)$ Boden in helle, mittlere und dunkle Bodentypen.

\begin{tabular}{lcc}
\hline & $\rho_{b, n}$ & $\rho_{b, t}$ \\
\hline hell & 0.18 & 0.35 \\
mittel & 0.10 & 0.20 \\
dunkel & 0.07 & 0.15 \\
\hline
\end{tabular}

Der Parameter $\mathrm{x}$ steuert den linearen Übergang von der Albedo für nassen Boden $\left(W_{b}=W_{b, \max }, x=1\right)$ zur Albedo eines Bodens, der bis auf $d_{\text {top }}=10 \mathrm{~cm}$ Tiefe ausgetrocknet ist $(x=0)$ :

$$
x=1-\frac{d_{r}}{d_{\text {top }}}\left(1-\frac{W_{b}}{W_{b, \max }}\right)
$$

Die Wolkenbedeckung $n_{w}$ bestimmt sich aus dem Verhältnis von aktueller zu potentieller PAR $r_{P A R}$ (Gleichung (2.58)): 


$$
n_{w}=\left\{\begin{array}{ccc}
1 & \text { wenn } & r_{P A R}<0.5 \\
\left(0.9-r_{P A R}\right) / 0.4 & \text { wenn } & 0.5<r_{P A R}<0.9 \\
0 & \text { wenn } & r_{P A R}>0.9
\end{array}\right.
$$

Mit $R_{n}=R_{n v}+R_{n b}$ und der Albedo der Oberfläche $\rho_{o}=1-a_{v}-a_{s}$ ergibt sich $R_{n}$ schließlich zu:

$$
R_{n}=R_{L \downarrow}-R_{L \uparrow}+\left(1-\rho_{o}\right) R_{s}
$$

Die thermische Strahlung von der Atmosphäre in Richtung der Oberfläche $R_{L \downarrow}$ wird berechnet aus dem temperatur- und feuchtigkeitsabhängigen Emissionsgrad der wolkenfreien Atmosphäre $\varepsilon_{A}$ und einem bewölkungsabhängigen Korrekturterm $r_{\varepsilon A}=1+0.22 n_{w}^{2}($ Bolz, 1949):

$$
R_{L \downarrow}=\varepsilon_{A} r_{\varepsilon A} \sigma T_{K}^{4}
$$

wobei $T_{K}$ die Temperatur in Kelvin angibt. $\sigma$ ist die Stefan-Boltzmann Konstante ( $5.6703 \cdot 10^{-8} \mathrm{~W} \mathrm{~m}^{-2} \mathrm{~K}^{-4}$ ). $\varepsilon_{A}$ berechnet sich aus dem Dampfdruck $e_{a}$ (siehe Gleichung (2.31)) und dem Standardwert für den Emissionsgrad der Atmosphäre $\varepsilon_{A 0}$, der nach Brutsaert (1982) bei 0.64 liegt (Knorr, 1997):

$$
\varepsilon_{A}=\varepsilon_{A 0}\left(\frac{e_{a}}{T_{K}}\right)^{\frac{1}{7}}
$$

Die thermische Strahlung von der Oberfläche zur Atmosphäre $R_{L \uparrow}$ wird aus der Temperatur und der Emissionsrate des Bodens $\varepsilon_{O}$ berechnet:

$$
R_{L \uparrow}=\varepsilon_{O} \sigma T_{K}^{4}
$$

$\varepsilon_{O}$ bestimmt sich aus dem Mittelwert für verschiedene Landoberflächen nach Brutsaert (1982) zu einem Wert von 0.97.

Der thermische Transmissionsgrad $t_{l, v}$ richtet sich direkt nach der aktuellen Vegetationsdichte über den Bedeckungsgrad $f_{c}$ und den LAI $\Lambda$ : 


$$
t_{l, v}=f_{c} e^{-\frac{\Lambda}{f_{c}}}+\left(1-f_{c}\right)
$$

mit

$$
f_{c}=\left\{\begin{array}{cc}
\frac{f_{c, \text { max }} \Lambda_{\text {max }}}{\Lambda_{0}} & \Lambda_{\text {max }}<\Lambda_{0} \\
f_{c, \text { max }} & \Lambda_{\text {max }} \geq \Lambda_{0}
\end{array}\right.
$$

Für den maximalen Bedeckungsgrad $f_{c \text {, max }}$ wird ein Wert von 0.9 angenommen (Knorr, 1997). $\Lambda_{\max }$ ist der Maximalwert des LAI des aktuellen Jahres, $\Lambda_{0}$ wird konstant auf den Wert $3.0 \mathrm{~m}^{3} \mathrm{~m}^{-3}$ gesetzt. Damit sind zunächst die Grundlagen der Strahlungsbilanz des Modells dargelegt. Weitere wichtige Parametrisierungen werden in der folgenden Beschreibung der Photosyntheseformulierung beschrieben.

In BETHY/DLR wird zunächst die unlimitierte Photosyntheserate $A_{c, 0}$ bestimmt. Über die Energie- und Wasserbilanz werden eventuell auftretende Limitierungen berücksichtigt, die die stomatäre Leitfähigkeit $g_{s}$ beeinflussen. Aus dieser wird dann schließlich die limitierte Photosyntheserate $A_{c}$ errechnet. Dabei werden einige pflanzenspezifische Gegebenheiten betrachtet, die es notwendig machen, in einzelnen Parametern zwischen den betrachteten Vegetationstypen zu unterscheiden. Die im Modell verwendeten Vegetationstypen und die spezifischen Parameter sind in Tabelle 2.4 aufgelistet (Tabelle 2.4 stellt eine Erweiterung der in Knorr (1997) vorgestellten Vegetationstypen dar). Verfügbare Landbedeckungsklassifizierungen wie GLC2000 oder Globcover müssen für die Verwendung in dem Modell in diese modellinternen Klassen übersetzt werden. Dabei besteht die Möglichkeit je Pixel einen Primär- und einen Sekundärtyp zu definieren und entsprechend die einzelnen Gewichtungen festzulegen. Damit kann der Verwendung einer grob aufgelösten Landnutzungsklassifizierung bei der meist heterogenen Verteilung der Vegetation zumindest zu einem bestimmten Teil Rechnung getragen werden. Allerdings wird damit auch eine genaue Kenntnis der Vegetationsverteilung des Modellgebietes vorausgesetzt.

Eine Anpassung der Vegetationsparameter für die Verwendung im Arbeitsgebiet stellt die Veränderung der Werte für die Buschlandvegetation (BETHY/DLR Klasse 8) dar. Die in Tabelle 2.4 aufgeführten Werte entstammen den Arbeiten von Beerling und Quick (1995) und wurden für Xerophyten gemessen, also Pflanzentypen, die sich an extrem trockene Gegebenheiten angepasst haben und demnach bei vergleichsweise schlechten Bedingungen sehr effektiv Photosynthese betreiben können (Beerling und Quick, 1995). Da es sich dabei allerdings um einen sehr spezialisierten Pflanzentyp handelt, werden für die optimaleren 
Gegebenheiten im Arbeitsgebiet Werte für Buschlandvegetation in ariden und semi-ariden Gebieten verwendet (Wohlfahrt et al., 1999) $\left(V_{c, \max }=71 \mu \operatorname{mol}(\mathrm{CO} 2) \mathrm{m}^{-2} \mathrm{~s}^{-1}\right.$ und $J_{\max }=123$ $\left.\mu \mathrm{mol}(\mathrm{CO} 2) \mathrm{m}^{-2} \mathrm{~s}^{-1}\right)$. Eine solche regionale Anpassung der Vegetationsparameter sollte in künftigen Modellversionen automatisch erfolgen und kann beispielsweise über Klimaklassifikationen, wie die in Kapitel 2.2 vorgestellte Gauß-Krüger-Einteilung, erfolgen.

Tabelle 2.4: Vegetationstypen des Modells mit den spezifischen Parametern: $V_{m}$ : maximale Karboxylierungsrate bei $25^{\circ} \mathrm{C}\left[\mu \mathrm{mol}\left(\mathrm{CO}_{2}\right) \mathrm{m}^{-2} \mathrm{~s}^{-1}\right]$, für $\mathrm{C} 3: J_{m}$ : maximale Elektronentransportrate bei $25^{\circ} \mathrm{C}$ [ $\left.\mu \mathrm{mol}\left(\mathrm{CO}_{2}\right) \mathrm{m}^{-2} \mathrm{~s}^{-1}\right]$ für C4: k: $\mathrm{CO}_{2}$ Spezifität bei $25^{\circ} \mathrm{C}\left[\mathrm{mmol}\left(\mathrm{CO}_{2}\right) \mathrm{m}^{-2} \mathrm{~s}^{-1}\right], \mathrm{h}$ : Vegetationshöhe [m], dr: Wurzeltiefe [m], C4: C4-Mechanismus, ansonsten C3.

\begin{tabular}{|c|c|c|c|c|c|c|}
\hline Nummer & Vegetationstyp & $\mathrm{V}_{\mathrm{m}}$ & $\mathrm{J}_{\mathrm{m}} ; \mathrm{k}$ & $\mathrm{h}$ & $\mathrm{d}_{\mathrm{r}}$ & $\mathrm{C} 4$ \\
\hline 1 & Laubwald, immergrün, tropisch & 62 & 118 & 30,0 & 6,9 & \\
\hline 2 & Laubwald, sommergrün, tropisch & 90 & 179 & 15,0 & 3,7 & \\
\hline 3 & Laubwald, immergrün, gemäßigt & 41 & 82 & 15,0 & 3,0 & \\
\hline 4 & Laubwald, sommergrün, gemäßigt & 35 & 70 & 15,0 & 3,0 & \\
\hline 5 & Nadelwald, immergrün & 29 & 52 & 15,0 & 3,9 & \\
\hline 6 & Nadelwald, sommergrün & 53 & 95 & 15,0 & 1,5 & \\
\hline 7 & Buschland, immergrün & 52 & 102 & 1,0 & 3,5 & \\
\hline 8 & Buschland, sommergrün & 160 & 266 & 1,0 & 3,5 & \\
\hline 9 & Grasland, C3, kurz & 42 & 80 & 0,3 & 1,8 & \\
\hline 10 & Grasland, C3, lang & 42 & 80 & 2,0 & 1,8 & \\
\hline 11 & Grasland, C4, kurz & 8 & 140 & 0,3 & 1,8 & $\mathrm{x}$ \\
\hline 12 & Grasland, C4, lang & 8 & 140 & 2,0 & 1,8 & $\mathrm{x}$ \\
\hline 13 & Tundra & 20 & 37 & 0,3 & 0,5 & \\
\hline 14 & Sumpf & 20 & 37 & 0,3 & 0,5 & \\
\hline 15 & Kulturpflanzen & 117 & 220 & 0,6 & 1,8 & \\
\hline 16 & Kulturpflanzen, bewässert & 123 & 227 & 0,6 & 1,8 & \\
\hline 17 & Baumfrüchte, tropisch & 60 & 106 & 2,0 & 6,9 & \\
\hline 18 & Zitrusfrüchte & 60 & 106 & 2,0 & 3,7 & \\
\hline 19 & Baumfrüchte, sommergrün, gemäßigt & 123 & 227 & 2,0 & 3,0 & \\
\hline 20 & Zuckerrohr & 39 & 700 & 2,0 & 1,8 & $\mathrm{x}$ \\
\hline 21 & Mais & 39 & 700 & 2,0 & 1,8 & $\mathrm{x}$ \\
\hline 22 & Reis & 98 & 190 & 0,3 & 0,3 & \\
\hline 23 & Baumwolle & 123 & 227 & 2,0 & 2,0 & \\
\hline 24 & Zuckerrübe & 129 & 226 & 0,5 & 1,8 & \\
\hline 25 & Soja & 94 & 168 & 0,8 & 1,8 & \\
\hline 26 & Sonnenblume & 80 & 213 & 2,0 & 2,7 & \\
\hline 27 & Gerste & 68 & 169 & 1,2 & 1,8 & \\
\hline 28 & Weizen & 83 & 193 & 1,5 & 1,8 & \\
\hline 29 & Raps & 61 & 187 & 1,0 & 1,8 & \\
\hline 30 & Buche & 46 & 109 & 15,0 & 4,0 & \\
\hline 31 & Eiche & 40 & 72 & 15,0 & 4,0 & \\
\hline 32 & Fichte/Tanne & 10 & 24 & 15,0 & 2,8 & \\
\hline 33 & Kiefer & 17 & 30 & 15,0 & 4,0 & \\
\hline
\end{tabular}




\subsubsection{Die Photorespiration}

Die Photorespiration $R_{d}$ ist ein wesentlicher Bestandteil der Photosynthese bei der unter Aufnahme von Sauerstoff wieder Kohlenstoffdioxid an die Atmosphäre abgegeben wird. Dies tritt vor allem in Stresssituationen, wie bei zu hohen Bestandstemperaturen auf, wenn die Stomata geschlossen werden. Die Photorespiration steht der eigentlichen Funktionsweise der Photosynthese direkt entgegen und hat ihre Ursache in der fehlenden Unterscheidung des Enzyms RuBisCO (Ribulose-1,2-bisphosphat-carboxylase/-oxygenase) zwischen Kohlenstoff und Sauerstoff im Calvin-Zyklus der Photosynthese. Die Photorespiration oder Blattatmung $R_{d}$ ist direkt proportional zur maximalen Karboxylierungsrate $V_{m}$ (Farquhar et al., 1980) (Tabelle 2.4) und einer vom photosynthetischen Mechanismus abhängigen Proportionalitätskonstante $\xi$ mit dem Wert 0.011 für C3-Pflanzen und 0.042 für C4-Pflanzen (Knorr, 1997):

$$
R_{d}=\xi V_{m} f_{N} e^{\frac{\left(T_{v}-25\right) E}{298 R\left(T_{v}+273\right)}}
$$

$R$ ist die allgemeine Gaskonstante $\left(R=8314 \mathrm{~J} \mathrm{~K}^{-1} \mathrm{~mol}^{-1}\right)$. Der Skalierungsfaktor $f_{N}=K e^{-K l}$ (mit dem Extinktionskoeffizienten $K=0.5 \mu$ ) berücksichtigt zusätzlich die Stickstoff- bzw. RuBisCO-Verteilung innerhalb des Blattbestandes, aufgeteilt in einzelne Bestandsschichten l. E gibt die Aktivierungsenergie von $50967 \mathrm{~J} \mathrm{~mol}^{-1}$ an (Collatz et al., 1992). Die Photorespiration wird für die einzelnen Bestandsschichten $l$ berechnet und für jeden Zeitschritt über den gesamten Bestand integriert (Knorr, 1997):

$$
R_{d, c}=\int_{0}^{\Lambda} R_{d}(l) d l
$$

Die Photorespiration wird als negative Assimilationsrate für die folgende Berechnung der Brutto- und Nettoprimärproduktion benötigt.

\subsubsection{Die Bruttoprimärproduktion}

Als Bruttoprimärproduktion (engl.: Gross Primary Productivity, GPP) wird die gesamte Aufnahmerate an Kohlenstoffdioxid unter Berücksichtigung der Photorespiration während der Photosynthese bezeichnet. Die Formulierung der Photosynthese geht zurück auf die Beschreibung von Farquhar (1980) für C3-Pflanzen und einer Erweiterung von Collatz et al. (1992) für Pflanzen mit dem C4-Mechanismus. In dem enzymkinetischen Modell wird 
die aktuelle Kohlenstoff-Assimilationsrate $A$ entweder über die Limitierung der Elektronentransportrate oder die Limitierung des zur Karboxylierung notwendigen Enzyms Rubisco beschrieben. Dabei wird jeweils die stärkste der beiden Limitierungen verwendet.

$$
A=\min \left\{J_{C} ; J_{E}\right\}-R_{d}
$$

$R_{d}$ ist die Blattatmung, beschrieben im vorhergehenden Abschnitt. Da die Berechnung der Energie- und Wasserbilanz unter nicht-limitierten Bedingungen erfolgt, wird zunächst die potentielle Assimilationsrate berechnet und erst in einem zweiten Schritt die Wasser- und Lichtlimitierung berücksichtigt (Knorr, 1997). Im Folgenden wird zunächst der C3Formalismus behandelt, anschließend der C4-Formalismus.

Nach Knorr (1997) ergeben sich die maximalen Assimilationsraten $J_{E}$ und $J_{C}$ für den C3Formalismus zu:

$$
J_{E}=J \frac{C_{i}-\Gamma_{*}}{4\left(C_{i}+2 \Gamma_{*}\right)}
$$

und

$$
J_{C}=V_{m} \frac{C_{i}-\Gamma_{*}}{C_{i}+\kappa_{m}},
$$

mit $\kappa_{m}=K_{C}\left(1+O_{x} / K_{O}\right)$, wobei $K_{C}$ und $K_{O}$ die Michaelis-Menten-Konstanten für Kohlendioxid und Sauerstoff, $O_{x}$ den Partialdruck von Sauerstoff bezeichnen (Werte siehe Tabelle 2.5). Der $\mathrm{CO}_{2}$-Kompensationspunkt $\Gamma_{*}$ gibt die minimale $\mathrm{CO}_{2}$-Konzentration in der Umgebungsluft an, ab der Photosynthese betrieben wird und steht nach Farquhar (1980) in linearem Zusammenhang mit der Bestandstemperatur $T_{V}\left(\Gamma_{*}=1.7 T_{V}\right) . C_{i}$ bezeichnet die interzelluläre $\mathrm{CO}_{2}$-Konzentration. Im nichtlimitierten Fall beträgt diese bei C3-Pflanzen $87 \%$ der Konzentration freier Luft $\left(C_{a}=360 \mathrm{ppm}\right) . J$ ist die Elektronentransportrate und wird in Abhängigkeit der Rate an absorbierter PAR $I_{P A R}$ (siehe Gleichung (2.27)) berechnet:

$$
J=\frac{\alpha I_{P A R} J_{m}}{\sqrt{J_{m}^{2}+\alpha^{2} I^{2}}}
$$

Dabei ist $\alpha$ die Effizienz des Photoneneinfangs ( $\alpha=0.28$, Beerling und Quick (1995)). Die Temperaturabhängigkeit der maximalen Elektronentransportrate $J_{m}$ wird aus der Rate bei $25^{\circ} \mathrm{C}$ (Tabelle 2.4) und der Bestandstemperatur $T_{V}$ (Gleichung (2.24)) in ${ }^{\circ} \mathrm{C}$ berechnet: 


$$
J_{m}\left(T_{V}\right)=J_{m}\left(25^{\circ} C\right) \frac{T_{V}}{25}
$$

Tabelle 2.5: Variablen zur Berechnung der Photosyntheserate nach Farquhar et al. (1980) (C3) und Collatz et al. (1992) (C4). $O_{x}$ ist der Partialdruck von Sauerstoff, $K_{C}$ und $K_{O}$ die Michaelis-Menten Konstanten für Kohlenstoff und Sauerstoff. $V_{m}$ ist die maximale Karboxylierungsrate und $k$ die $\mathrm{CO}_{2}$ Spezifität mit den pflanzenspezifischen Werten aus Tabelle 2.4. $E$ gibt die Werte der Aktivierungsenergie in $\mathrm{J} \mathrm{mol}^{-1}$ an.

\begin{tabular}{llll}
\hline Variable & Wert bei $25^{\circ} \mathrm{C}$ & Einheit & $E$ \\
\hline$O_{x}$ & 0.21 & $\mathrm{~mol} \mathrm{~mol}^{-1}$ & 35948 \\
$K_{C}$ & 460 & $\mu \mathrm{mol} \mathrm{mol}^{-1}$ & 59356 \\
$K_{O}$ & 0.33 & $\mathrm{~mol} \mathrm{~mol}^{-1}$ & 35948 \\
$V_{m}$ & s. Tabelle 2.4 & $\mu \mathrm{mol} \mathrm{m}^{-2} \mathrm{~s}^{-1}$ & 58520 \\
$k$ & s. Tabelle 2.4 & $\mathrm{mmol} \mathrm{m}^{-2} \mathrm{~s}^{-1}$ & 50967 \\
\hline
\end{tabular}

Bei ausreichender Lichtzufuhr ist die Photosyntheserate durch die maximale Durchsatzrate $V_{m}$ des Enzyms Rubisco limitiert, das die Aufnahme von Kohlenstoffdioxid aus der Umgebungsluft ermöglicht. Erst bei schlechteren Lichtverhältnissen wird die Photosynthese durch die Elektronentransportrate limitiert. Für den Wechsel zwischen rubisco- und lichtlimitierter Photosynthese wird nach Farquhar et al. (1980) und Beerling und Quick (1995) ein abrupter Übergang angenommen. Bei dichten Blattbeständen nimmt die Lichtverfügbarkeit vom Kronendach zur Untergrenze ab. Daran passen die Pflanzen den Stickstoffgehalt und damit den Gehalt an Rubisco entsprechend an. Nach Sellers et al. (1996) wird ein exponentieller Verlauf für $J_{m}$ und $V_{m}$ angenommen, beschrieben durch den Skalierungsfaktor $f_{N}=K e^{-K l}$. Diese Formulierung wird für Bäume, Sträucher und Feldfrüchte mit $\Lambda>\Lambda_{0}=3$ verwendet (Knorr, 1997).

Weitere Temperaturabhängigkeiten werden durch die Formulierung

$$
k\left(T_{V}\right)=k\left(25^{\circ} C\right) e^{\frac{\left(T_{V}-25\right) E}{298 R\left(T_{V}+273\right)}}
$$

errechnet, wobei k für den jeweiligen Parameter steht. $E$ ist die entsprechende Aktivierungsenergie (Tabelle 2.5). Die Bestandstemperatur unterscheidet sich von der Temperatur der Umgebungsluft. Diese ist von der Energiebilanz des Blattbestandes der Vegetation abhängig (Knorr, 1997):

$$
T_{v}=T+\frac{R_{n v}-E_{t}}{\rho_{l} c_{p} G_{a}}
$$


Dabei ist $c_{p}=1005.46 \mathrm{~J} \mathrm{~kg}^{-1} \mathrm{~K}^{-1}$ die spezifische Wärme von Luft bei konstantem Druck $p$, $\rho_{l}=1.29 \mathrm{~kg} \mathrm{~m}^{-3}$ die Dichte der Luft und $G_{a}$ die aerodynamische Leitfähigkeit die sich aus der Bestandshöhe $h_{v}$ (beziehungsweise der Rauhigkeitslänge $0.1 h_{v}$ ) und der Windgeschwindigkeit $u$ berechnet:

$$
G_{a}=\frac{\kappa u}{\ln \left(\frac{10}{0.1 h_{v}}+1\right)^{2}}
$$

$\kappa$ ist die von-Kármán-Konstante $(\kappa=0.41)$, durch die der turbulente Luftstrom an der Grenzfläche zwischen Blattbestand und Umgebungsluft berücksichtigt wird (Knorr, 1997).

Schließlich ergibt sich die nichtlimitierte Photosynthese über die einzelnen Bestandsschichten summiert zu:

$$
A_{c, 0}=\sum_{l=0}^{\Lambda} A_{0}\left(I_{P A R}(l)\right)
$$

Dabei wird jeweils die absorbierte PAR je Schicht bestimmt über

$$
\left.I_{P A R}(l)=\frac{1}{l_{k}-l_{k-1}}\left\{R\left(l_{k-1}\right)+R_{\downarrow}\left(l_{k-1}\right)-R_{\uparrow}\left(l_{k-1}\right)\right]-\left[R\left(l_{k}\right)+R_{\downarrow}\left(l_{k}\right)-R_{\uparrow}\left(l_{k}\right)\right]\right\}
$$

wobei die direkte Einstrahlung $R(l)=R(0) e^{-K l}\left(R(0)=d_{P A R} R_{P A R}\right)$ sowie die abwärts und aufwärts gerichteten diffusen Flüsse $R_{\downarrow}(l)$, und $R_{\uparrow}(l)$ eingehen. Hier werden die Lösungen der Zweistromapproximation nach Sellers (1985) verwendet.

Mit dieser unlimitierten Photosyntheserate wird in einem nächsten Schritt die stomatäre Leitfähigkeit mit $C_{i, 0}=0.87 C_{a}$ für C3-Pflanzen und $C_{i, 0}=0.67 C_{a}$ für C4-Pflanzen (Morison, 1987; Schulze et al., 1994), als

$$
g_{s, 0}(l)=\frac{1.6 A_{c, 0}(l)}{C_{a}-C_{i, 0}} \frac{R T}{p}
$$

berechnet und über den Blattbestand zur Bestandsleitfähigkeit $G_{c}$ aufsummiert. Diese wird benötigt, um die potentielle Transpiration $E_{t, \max }$ der Vegetation nach dem Penman-Monteith Model (Monteith, 1965) zu bestimmen: 


$$
\lambda E_{t, \max }=\frac{s R_{n v}+\rho c_{p} \Delta e G_{a}}{s+\gamma\left(1+\frac{G_{a}}{G_{c, 0}}\right)}
$$

$s$ ist die Steigung der Dampfdruckkurve $\left(s=\partial e_{s}(T) / \partial T\right)$ und $\Delta e=e_{s}(T)-e_{a}$ das Dampfdruckdefizit mit

$$
e_{s}(T)= \begin{cases}610.78 e^{\frac{17.269 T}{237.3+T}} & T>0 \\ 610.78 e^{\frac{22.33 T}{231.15+T}} & T<0\end{cases}
$$

dem Sättigungsdampfdruck über Wasser oder Eis (Murray, 1967) und dem täglichen Gang des Wasserdampfes

$$
e_{a}=e_{a 0}+f_{e} h\left(e_{s}(T)-e_{a 0}\right)
$$

mit dem Standardwert $e_{a 0}=\left(h_{0}+\left(1-h_{0}\right) f_{e}\right) e_{s}\left(T_{\min }\right)$ (Knorr, 1997). Nach Gleichung (2.24) setzt sich die aktuelle Evapotranspiration $E$ zusammen aus den Anteilen des Bodens $E_{b}$, der Vegetation $E_{i}+E_{t}$ und des eventuell vorhandenen Schnees $E_{s}$. Daraus ergibt sich schließlich das Verhältnis von aktueller zu potentieller Evapotranspiration:

$$
f_{e}=\frac{E}{E_{t, \text { max }}+E_{v, \text { max }}+E_{s, \text { max }}+E_{s n, \text { max }}}
$$

Mit den Werten $f_{e}=1$ und $f_{e}=0$ wird demnach entsprechend zwischen feuchten und trockenen Bedingungen unterschieden. Die Größe $h$ in Formel (2.31) bezeichnet das Tagesmaximum des Dampfdruckes bei maximaler Feuchte. $h_{0}$ ist die relative Feuchte bei Sonnenaufgang $\left(T=T_{\min }\right)$ und absoluter Trockenheit. Für die Berechnung der aktuellen Transpirationsrate der Vegetation wird auch die aktuelle Bestandsleitfähigkeit (limitiert) $G_{c}$ benötigt (Knorr, 1997):

$$
G_{c}=G_{c, 0} \frac{1}{1+b_{e} \Delta e}
$$

Nach dem Modell von Federer $(1979,1982)$ wird der Kontrollparameter $b_{e}$ in einem täglichen Zeitschritt neu berechnet, so dass zur Zeit der höchsten, maximalen Transpirationsrate $E_{t, \text { max }}$ die aktuelle Transpirationsrate $E_{t}$ geringer oder gleich der Wasserversorgungsrate 
$S$ ist. Diese Rate ist über den Tag konstant und hängt vom Bodenwassergehalt $W_{s}^{\text {eff }}$ und der maximalen Wurzelversorgungsrate $c_{w}=0.5 \mathrm{~mm} \mathrm{~h}^{-1}$ ab (Knorr, 1997):

$$
S=c_{w} \frac{W_{s}^{e f f}}{W_{s, \max }}
$$

Zunächst wird entsprechend die aktuelle, limitierte Evapotranspirationsrate berechnet. Diese bestimmt sich zunächst aus der Nettoeinstrahlung $R_{n v}$ und wird durch das Bodenwasser $W_{b}$ und das Blätterreservoir $W_{i}$ beschränkt. Bei nasser Blattoberfläche wird hier die Bestandsleitfähigkeit $G_{c}$ als unendlich angenommen, so dass die Verdunstung maximal wird und das gesamte verdunstete Wasser von der Blattoberfläche kommt:

$$
E_{v}=E_{i}=E_{v, \max }=\frac{s R_{n v}+\rho c_{p} \Delta e G_{a}}{s+\gamma}
$$

Transpiration der Pflanzen ist nur bei trockener Vegetation möglich und wird dann durch $G_{c}$ bestimmt (Monteith, 1965):

$$
E_{v}=E_{t}=\frac{s R_{n v}+\rho c_{p} \Delta e G_{a}}{s+\gamma\left(1+\frac{G_{a}}{G_{c}}\right)}
$$

Die resultierende tägliche Transpirationsrate $E_{v} \Delta t$ wird schließlich über einen mittleren Feuchteanteil

$$
F_{i}=\frac{E_{v}(t) \Delta t}{\int_{T a g} E_{v, \max }\left(t^{\prime}\right) d t^{\prime}}
$$

berechnet, wodurch diese sich entsprechend reduziert (Knorr, 1997):

$$
E_{t}(t) \Delta t=\left(1-F_{i}\right) \int_{T a g} E_{t}(t) d t
$$

Zur Berechnung des Kontrollparameters $b_{e}$ wird diese Gleichung nach $G_{c}$ aufgelöst und in Gleichung (2.33) eingesetzt. Schließlich kann damit die aktuelle Bestandsleitfähigkeit selbst berechnet werden.

Die limitierte Photosyntheserate ergibt sich schließlich mit $G_{C}^{\prime}=0.625 G_{C} p / R T \mathrm{zu}$ 


$$
A_{C}=G_{C}^{\prime}\left(C_{a}-C_{i}\right)
$$

Außerdem gilt nach Gleichung (2.18) entweder $A_{C}=J_{E}-R_{d}$ oder $A_{C}=J_{C}-R_{d}$. Umstellen von Gleichung (2.39) nach $C_{i}$ und Einsetzen in $J_{E}$ bzw. $J_{C}$ (Gleichungen (2.19) und (2.20)) ergibt eine quadratische Gleichung

$$
0=J_{E}^{2}-\underbrace{\left[R_{d}+\frac{J}{4}+G_{C}^{\prime}\left(C_{a}+\frac{\Gamma_{*}}{2}\right)\right]}_{b} J_{E}+\underbrace{\frac{G_{C}^{\prime}}{4}\left(C_{a}-\Gamma_{*}\right) J+\frac{R_{d}}{4} J}_{c}
$$

bzw.

$$
0=J_{C}^{2}-\underbrace{\left[R_{d}+V_{m}+G_{C}^{\prime}\left(C_{a}+V_{m}\right)\right]}_{b} J_{C}+\underbrace{G_{C}^{\prime}\left(C_{a}-\Gamma_{*}\right) V_{m}+R_{d} V_{m}}_{c}
$$

Das Minimum der beiden Lösungen $J_{1 / 2}=-\frac{b}{2} \pm \sqrt{\frac{b_{2}}{4}-c}$ wird zur Berechnung der Photosyntheserate verwendet. Um schließlich auf die tägliche Bruttoprimärproduktion GPP zu kommen, wird sowohl die Photosyntheserate als auch die Dunkelatmung über die Bestandsschichten aufsummiert. Das Integral über einen Tag über die Summe der beiden Größen entspricht dann der GPP (Knorr, 1997):

$$
G P P=\int_{1 T a g}\left(A_{c}\left(t^{\prime}\right)+R_{d, c}\left(t^{\prime}\right)\right) d t^{\prime}
$$

Im Falle des C4-Mechanismus ändert sich das Verfahren gemäß Collatz et al. (1992). Die Photosyntheserate wird nach wie vor über die Dunkelatmung und das Minimum von $J_{E}$ und $J_{C}$ bestimmt. Die Berechnung von $J_{E}$ und $J_{C}$ im nichtlimitierten Fall erfolgt nun über:

$$
\begin{gathered}
J_{E}=\frac{1}{2 \theta}\left[\alpha_{i} I+V_{m}-\sqrt{\left(\alpha_{i} I+V_{m}\right)^{2}-4 \theta V_{m} \alpha_{i} I}\right] \\
J_{C}=k C_{i}
\end{gathered}
$$

mit

$$
k=J_{m} 1.0 \cdot 10^{3} f_{N} e^{\frac{E\left(T_{V}-25\right)}{298 R\left(T_{V}+273\right)}} .
$$


$\theta$ ist ein Kurvenparameter für $J_{E}$ mit konstantem Wert 0.83 und $\alpha_{i}=0.04$ die Effizienz des Quanteneinfangs für C4-Pflanzen. Aus der nicht-limitierten Photosyntheserate $A_{c, 0}$ berechnet sich analog zum C3-Fall die stomatäre Leitfähigkeit bzw. die Bestandsleitfähigkeit $G_{c}$ und schließlich die aktuelle Evapotranspiration $E_{t}$ (Knorr, 1997). Die limitierte Photosyntheserate $A_{c}$ bestimmt sich letztlich über Gleichung (2.19) für $J_{E}$ und Gleichung (2.46) für $J_{C}$.

$$
J_{C}=\frac{1}{1+\frac{G_{c}^{\prime}}{k}}\left(G_{c}^{\prime} C_{a}+R_{d}\right)
$$

Aus den berechneten Werten für $J_{E}$ und $J_{C}$ werden in beiden Mechanismen (C3 und C4) die Assimilationsraten $A_{c}$ bestimmt, wobei die Limitierung durch zu hohe Temperaturen berücksichtigt wird. Diese Limitierung tritt für Temperaturen oberhalb von $55^{\circ} \mathrm{C}$ in $\mathrm{Kraft}$ (Collatz et al, 1991) über die Funktion

$$
\eta=\frac{1}{1+e^{T_{v}-55}}
$$

Diese Limitierung trägt dem Schutzmechanismus der Pflanzen Rechnung, zu starken Flüssigkeitsverlust bei hohen Temperaturen durch ein Verschließen der Stomata zu vermeiden. Dementsprechend verringert sich dadurch ebenfalls die Umsatzrate der Pflanzen.

\subsubsection{Die Nettoprimärproduktion}

Die Nettoprimärproduktion beschreibt den aufgenommenen Kohlenstoffanteil, der nach Berücksichtigung der autotrophen Respiration $R_{a}$ also dem Anteil an $\mathrm{CO}_{2}$ der während der Photosynthese an die Atmosphäre zurückgegeben wird, in der Pflanze als Zuwachs an Biomasse von der gesamten GPP zurückbleibt:

$$
N P P=G P P-R_{a}
$$

Die autotrophe Respiration setzt sich aus der Erhaltungsatmung $R_{E}$ und der Wachstumsatmung $R_{W}$ zusammen:

$$
R_{a}=R_{E}+R_{W}
$$

Für die Berechnung der Erhaltungsatmung wird angenommen, dass diese typischerweise $\mathrm{zu} 40 \%\left(f_{R, \text { Blatt }}=0.40\right)$ in den Blättern stattfindet (Ryan, 1991) und damit über die Dunkelatmung $R_{d, c}$ bestimmt werden kann: 


$$
R_{E}=\frac{R_{d, c}}{f_{R, \text { Blatt }}}
$$

Auf die Wachstumsatmung entfällt ein Anteil von $f_{R, W}=0.25$ an dem Kohlenstoffgehalt der produzierten Biomasse (Ryan, 1991). Daraus ergibt sich mit $R_{W}=f_{R, W} N P P$ :

$$
R_{W}=\frac{f_{R, W}}{1+f_{R, W}}\left(G P P-R_{E}\right)
$$

und damit die NPP zu:

$$
N P P=\frac{1}{1+f_{R, W}}\left(G P P-R_{E}\right)
$$

Die Wachstumsatmung erhält allerdings nur einen Beitrag, solange die NPP einen positiven Wert hat, also wenn gilt: $\left(G P P-R_{E}\right) \geq 0$.

Neben GPP und NPP werden auch die aktuelle Evapotranspiration (Gleichung (2.4) sowie das berechnete Bodenwasser vom Modell ausgegeben. Die Berechnung des Bodenwassers erfolgte in der ursprünglichen Version von BETHY/DLR mittels eines stark vereinfachten, einschichtigen Modells. Durch dieses Modell wurde die Speicherfähigkeit der unterschiedlichen Bodentypen unterschätzt. Vor allem in Regionen mit niedrigen Niederschlagsraten führte dies zu einer Simulation lange anhaltender Trockenheit, da das eingehende Wasser zu schnell versickert war. Daher wurde parallel zu dieser Arbeit durch Tum et al. (2012) ein mehrschichtiges Bodenmodell implementiert, das die Speicherfähigkeit von bis zu sieben Bodenschichten mit Hilfe von van Genuchten-Parametern bestimmt (van Genuchten, 1980). Allerdings wurde dabei wiederum nicht die Möglichkeit einer Austrocknung des Bodens berücksichtigt, was bei der Anwendung dieses Modells in ariden Gebieten unbedingt notwendig ist. Im Rahmen der vorliegenden Arbeit wurde daher eine Erweiterung des Bodenmodells nach Williams et al. (1984) durchgeführt, durch die auch Stressfaktoren wie Dürreperioden und die Verringerung des pflanzenverfügbaren Bodenwassers berücksichtigt werden. Nach dem Ansatz steht der Pflanze solange ausreichend Wasser zur Verfügung, solange mehr als 25\% des maximalen, pflanzenverfügbaren Wassergehaltes des Bodens vorhanden sind. Dieser maximale, pflanzenverfügbare Wassergehalt wird als nutzbare Feldkapazität $F C$ bezeichnet. Sind weniger als $25 \%$ dieses Wassergehaltes vorhanden, wird angenommen, dass nur noch ein gewisser Anteil des Bodenwassers durch die Pflanze aufgenommen werden kann (Gleichung (2.53)). Dadurch werden Adhäsionskräfte berücksichtigt, die einen bodenspezifischen Anteil an Wasser in kleinen Poren des Bodens halten. 


$$
W=\left\{\begin{array}{ccc}
W_{s} & \text { wenn } & W_{s}>0.25 F C \\
W_{s}\left(\frac{W_{s}}{0.25 F C}\right) & \text { wenn } & W_{s} \leq 0.25 F C
\end{array}\right.
$$

Zusammenfassend geht die Berechnung der Kohlenstoffassimilationsrate in der Vegetation im Wesentlichen auf einige meteorologische (Niederschlag, $N$, Temperatur, $T$, Windgeschwindigkeit, $u$, photosynthetisch aktive Strahlung, PAR), der phänologischen Zeitreihe des LAI, $\Lambda$, sowie konstante pflanzenphysiologische Parameter zurück (siehe Tabelle 2.4 und Tabelle 2.5). Diese Eingangsgrößen sowie die Landbedeckungsklassifikation werden im folgenden Kapitel näher beschrieben und durch den Vergleich mit Bodenmessungen validiert. 


\subsection{Datengrundlage}

Da die ursprüngliche Version des Modells BETHY als Unterprogramm für die Wechselwirkung zwischen Atmosphäre und Vegetation des auf globaler Skala verwendeten Klimamodells ECHAM (ECMWF in Hamburg) (DKRZ, 1993; Knorr, 1997) konzipiert wurde, mussten in vorherigen Arbeiten (Wisskirchen, 2005) sowie während dieser Arbeit Anpassungen an regionale Anforderungen durchgeführt werden. Als wesentliche Änderung des Modells durch Wisskirchen (2005) ist die Umstellung der Eingangsdaten auf hauptsächlich fernerkundungsbasierte Daten hervorzuheben. Zuvor wurde für Niederschlag, Temperatur und Elevation die Klimatologie nach Leemans und Cramer (1991) verwendet, die sich auf Monatsmittel von weltweit 1057 Messstationen aus den Jahren 1931-1960 stützt. Die ursprüngliche Auflösung von $0.5 \mathrm{Grad}$, die sich aus den verwendeten Eingangsdaten ergibt, ist für nationale oder regionale Anwendungen nicht geeignet, da vor allem meteorologische Größen wie Niederschlag und Temperatur auf vergleichsweise kleinen Skalen stark variieren können. Zudem ist selten eine Vegetationsverteilung zu beobachten, bei der auf einem Gebiet von über 50x50 km nur ein einheitlicher Vegetationstyp vertreten ist. Zwar können mit dem Modell je Pixel zwei Vegetationstypen mit entsprechendem Mischungsverhältnis berücksichtigt werden, dennoch ist auf der betrachteten Skala selten eine Vegetationsverteilung von nur zwei Spezies zu finden. Demnach musste neben der höheren Auflösung der Klimadaten auch die Landnutzungsklassifikation angepasst werden. Dabei wurde das Vorhaben verfolgt, möglichst auf global frei verfügbare Datensätze zurückzugreifen, um einen räumlich flexiblen Einsatz des Modells zu gewährleisten.

\subsubsection{Meteorologischer Antrieb}

Als klimatische Eingangsdaten werden Zeitreihen von Lufttemperatur, Niederschlag, Windgeschwindigkeit und Wolkenbedeckung benötigt. Bezogen werden diese aus dem ECMWF-Interim-Datensatz, welche aus der Analyse eines weltweiten Netzes meteorologischer Messstationen auf einem Gitter von $0.25^{\circ}$ x $0.25^{\circ}$ räumlicher Auflösung berechnet werden. Die Tagesniederschlagswerte werden von dem ECMWF Re-Analyse Datensatz ERA-Interim verwendet, der mit Hilfe eines Wettermodells erstellt wird, in das operationell unterschiedliche Beobachtungsdaten eingehen, über die die jeweiligen Vorhersagen kontinuierlich korrigiert werden. Die Beobachtungsdaten umfassen sowohl in-situ Messungen von Wetterstationen, Bojen und Radiosonden als auch Beobachtungen aus der Fernerkundung. Die räumliche Auflösung ist für alle Produkte $0.25^{\circ}$ x $0.25^{\circ}$ (ca. $25 \mathrm{~km}$ x 25 $\mathrm{km}$ am Äquator). Verwendet werden Datensätze zur Temperatur in zwei Metern Höhe über dem Boden, die Windgeschwindigkeit, der Niederschlag und der Bewölkungsgrad in drei 
Höhenschichten. Mit Hilfe letzterem wird, wie später beschrieben, die photosynthetisch aktive Strahlung an der Erdoberfläche berechnet.

Zur quantitativen Bewertung der meteorologischen Eingangsparameter kann auf ein Netzwerk von 760 Stationen innerhalb Südafrikas zurückgegriffen werden. Für die weiteren Regionen im Arbeitsgebiet sind solche Daten leider nicht verfügbar. Eine Verteilung der Messstationen zeigt Abbildung 2.3, wobei eine eindeutige Häufung um Kapstadt im Südwesten der Provinz Westkap sowie im Kruger Nationalpark im Nordosten erkennbar ist.

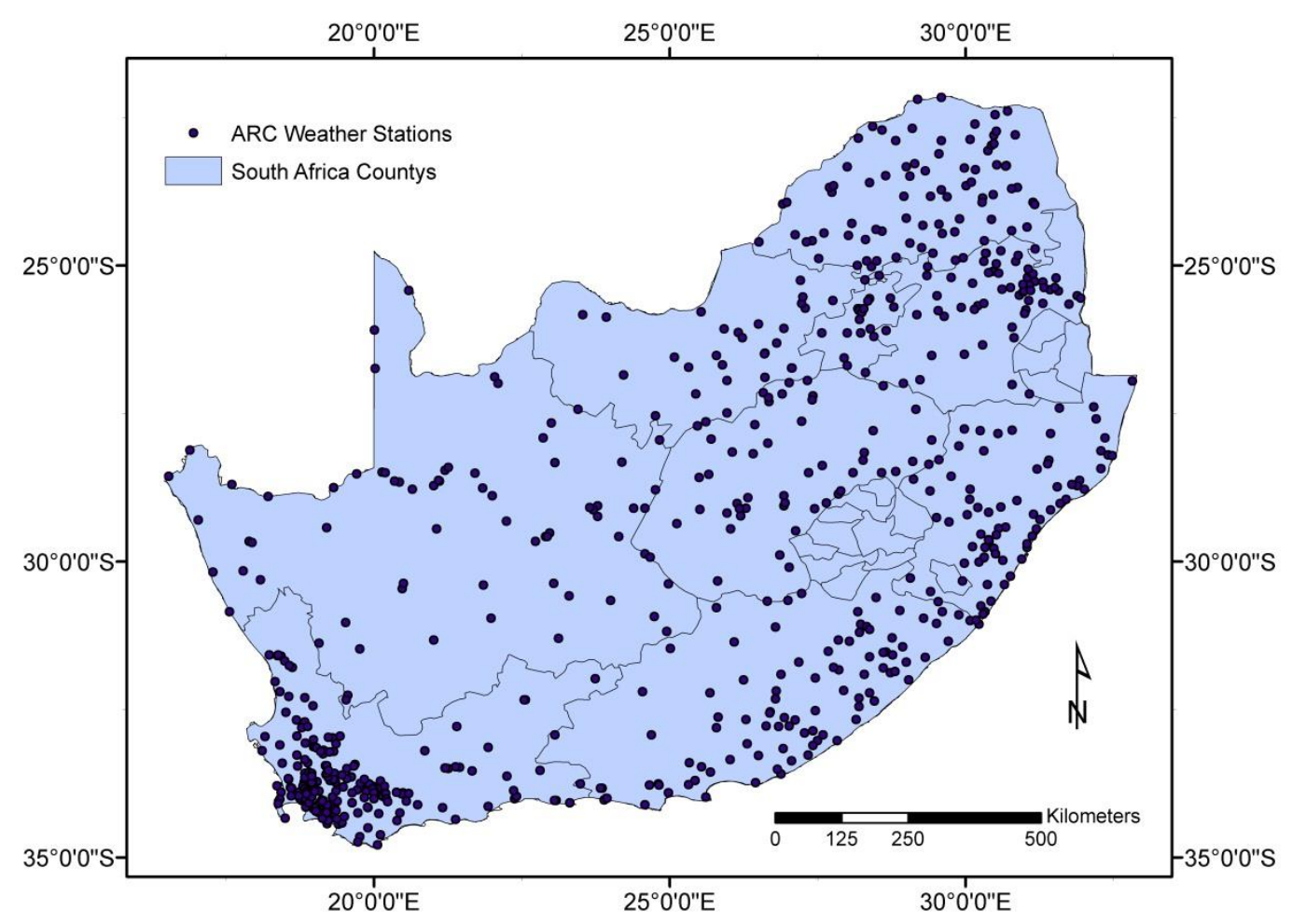

Abbildung 2.3: Verteilung der 760 meteorologischen Messstationen des ARC über Südafrika.

In den weniger dicht besiedelten Gebieten im Nordwesten sind nur vereinzelt Stationen zu finden, vorzugsweise entlang von Flussläufen (beispielsweise dem Fluss Orange entlang der Grenze zu Namibia). Die Daten wurden vom südafrikanischen Agricultural Research Council (ARC) für diese Arbeit zur Verfügung gestellt. Zu den Messungen zählen alle im Modell verwendeten meteorologischen Größen: maximale und minimale Temperatur, Niederschlagssumme, PAR und Windgeschwindigkeit. In der Regel liegen tägliche Messungen vor, allerdings kam es häufiger zu Ausfällen der Geräte. Vor allem bei abseits gelegenen Stationen führte dies teilweise zu längeren Intervallen unphysikalischer Messwerte. Daher mussten die Datensätze zunächst aufbereitet werden, um Messfehler zu identifizieren und zu entfernen. Dennoch standen nach der Korrektur für jede Messgröße jeweils über eine 
Million Messwerte zur Verfügung. Anschließend konnten, sofern Messwerte vorhanden waren, diese mit den ECMWF-Werten verglichen werden. Dies wird im Folgenden für die einzelnen Parameter dargestellt.

\subsubsection{Der Niederschlag}

Die ECMWF-Niederschlagswerte sind jeweils als akkumulierte Werte zu zwei Zeitpunkten am Tag verfügbar (0:00Uhr und 12:00 Uhr). Da der Wasserhaushalt im Modell in täglichem Zeitschritt berechnet wird, werden die beiden Werte zu einer Tagessumme als täglicher Eintrag in das System zusammengefasst.

Für den quantitativen Vergleich der Niederschlagsdaten standen Messungen von 568 Stationen zur Verfügung. Abbildung 2.4a zeigt die Häufigkeitsverteilung der Korrelationskoeffizienten der einzelnen Stationen, Abbildung $2.4 \mathrm{~b}$ das Streudiagramm aller Messwerte. Mit einer Korrelation von 0.49 fällt die Qualität der Niederschlagswerte des ECMWF äußerst schlecht aus. Zwar ist eine Häufung bei höheren Werten über 0.5 zu erkennen, im Streudiagramm wird dagegen kein Zusammenhang zwischen den Messdaten und den Werten des ECMWF deutlich.

(a)

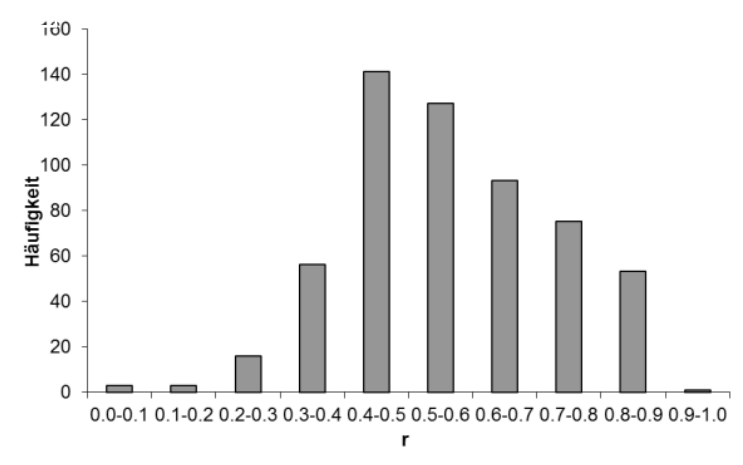

(b)

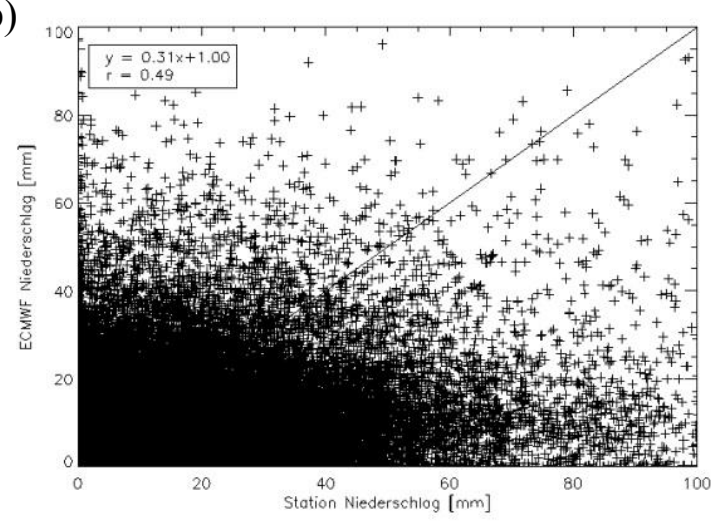

Abbildung 2.4: Verteilung der Korrelationskoeffizienten der einzelnen ARC-Messstationen (a) und das Streudiagramm aller Messwerte (b) für den Niederschlag.

Hier wurden zunächst die Tageswerte des Niederschlags der beiden Datenquellen verglichen. Wie später diskutiert wird, ist in der Formulierung des Bodenmodells der genaue Zeitpunkt des Wassereintrages nicht ausschlaggebend, solange eine ausreichende Mindestmenge an Feuchte im Boden vorhanden ist, um die Vegetation zu versorgen. In den betrachteten Trockengebieten ist dies in der Regel während der Regenzeit der Fall. Demnach können die Monatssummen der Niederschlagsmenge bestimmt und verglichen werden (Abbildung 2.5). Aus den Werten aller Stationen ist gegenüber dem Vergleich aller Tagesmessungen zunächst eine geringere Streuung zu beobachten. Die Korrelation fällt in 
diesem Vergleich mit 0.59 höher aus, dennoch wird die Niederschlagssumme durch die ECMWF-Werte, in Relation zu den ARC-Messwerten, im Mittel um fast $60 \%$ unterschätzt.

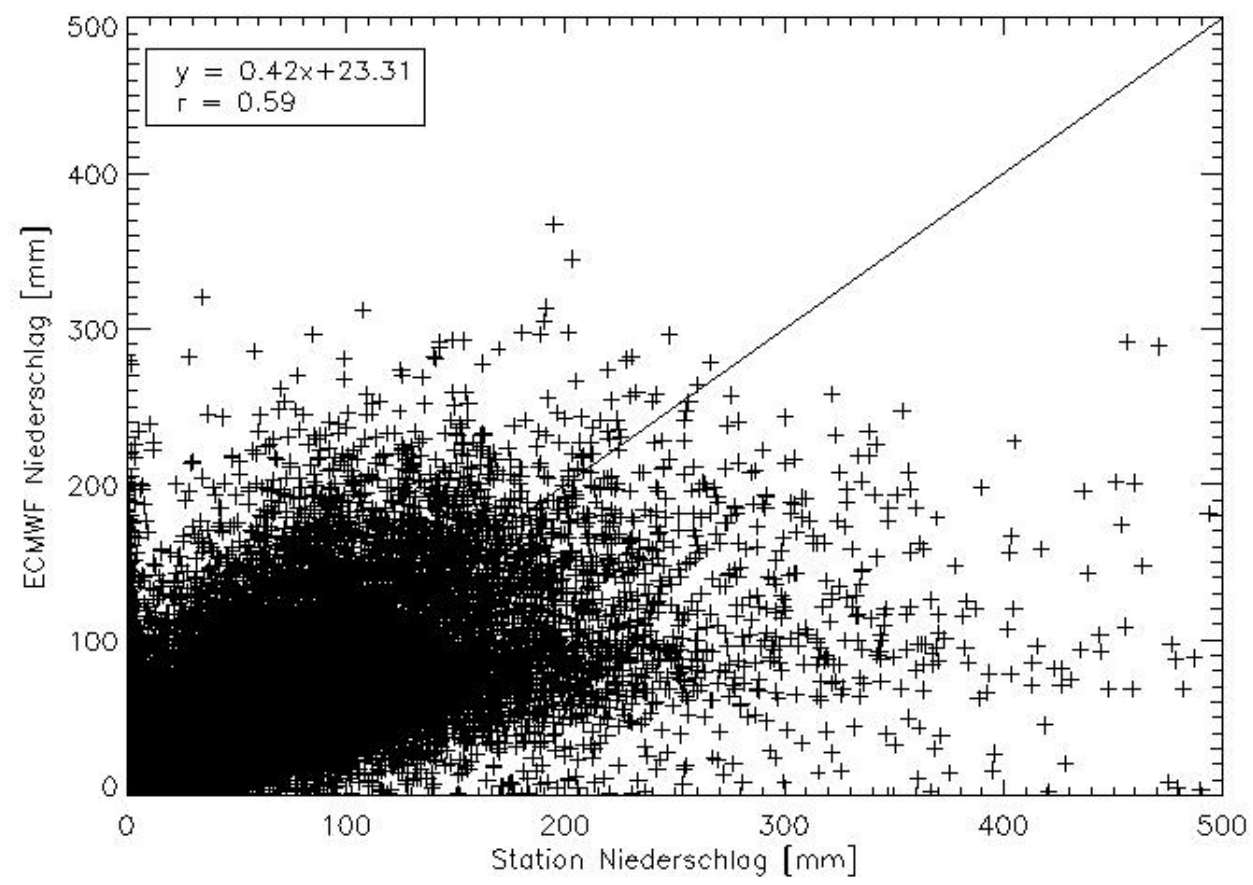

Abbildung 2.5: Streudiagramm der Monatssummen des Niederschlags aller ARC-Messstationen gegen die Werte des ECMWF.

\subsubsection{Die Temperatur}

Die Temperatur ist täglich als Minimal-, Maximal- und mittlere Temperatur verfügbar. Für den Tagesgang wird das Maximum für 14:00 Uhr Modellzeit angenommen, das Minimum bei Sonnenaufgang. Bis Sonnenuntergang verläuft die Temperatur sinusförmig, nachts mit linearem Abfall. Die Temperatur ist ein für die Photosynthese ausschlaggebender Parameter. Daher ist es von Vorteil, diesen auf eine höhere Auflösung zu interpolieren. Dazu wird ein adiabatischer Ansatz verwendet, über den die Temperatur mittels eines höher aufgelösten digitalen Geländemodells (Digital Elevation Model, DEM) an die tatsächliche Höhe der einzelnen Punkte angepasst wird. Verwendet wird dabei der Gradient $a$ der U.S. Standardatmosphäre von Normal-Null bis zur Tropopause $(-6.5 \mathrm{~K} / 1000 \mathrm{~m})$. Als DEM findet das GTOPO30-Produkt in 30" x 30" Auflösung (ca. $1 \mathrm{~km} \mathrm{x} 1 \mathrm{~km}$ am Äquator) Anwendung. Dieses wurde am USGS Center for Earth Resources Observation and Science (EROS) entwickelt (USGS, 1996). Das DEM wird auch für die höhenabhängige Berechnung des Druckes $p$ in BETHY/DLR verwendet, mittels der barometrischen Höhenformel: 


$$
p=p_{0}\left(1-\frac{a h}{T}\right)^{\frac{M_{l} g}{a R}}
$$

$p_{0}$ ist der Normaldruck $(1013,25 \mathrm{hPa}), M_{l}$ die molare Masse von Luft $\left(0,028964 \mathrm{~kg} \mathrm{~mol}^{-1}\right)$, $g$ die Schwerebeschleunigung $\left(9,81 \mathrm{~m} \mathrm{~s}^{-2}\right), R$ die universelle Gaskonstante $\left(8,314 \mathrm{~J} \mathrm{~K}^{-1}\right.$ $\left.\mathrm{mol}^{-1}\right), h$ die Höhe und $T$ die mittlere Temperatur.

Temperaturwerte von 760 Stationen standen für den Vergleich der Tagesminima und maxima der Temperatur zur Verfügung. Die sehr gute Korrelation der ECMWF-Werte mit denen der Messstationen ist bereits in den Häufigkeitsdiagrammen (Abbildung 2.6a und Abbildung 2.7a) zu erkennen. Über $60 \%$ der Werte für $T_{\min }$ haben ein $\mathrm{r}$ größer 0.9 , für $T_{\max }$ liegt der Anteil bei fast $80 \%$.

(a)

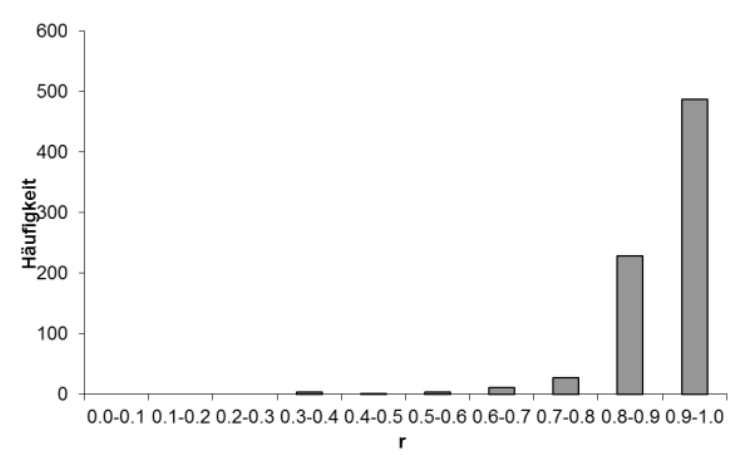

(b)

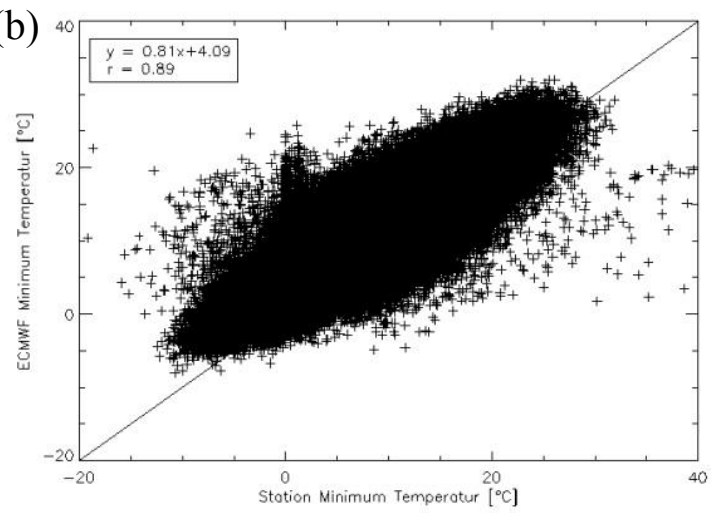

Abbildung 2.6: Verteilung der Korrelationskoeffizienten der einzelnen ARC-Messstationen (a) und das Streudiagramm aller Messwerte (b) für das Tagesminimum der Temperatur.

(a)

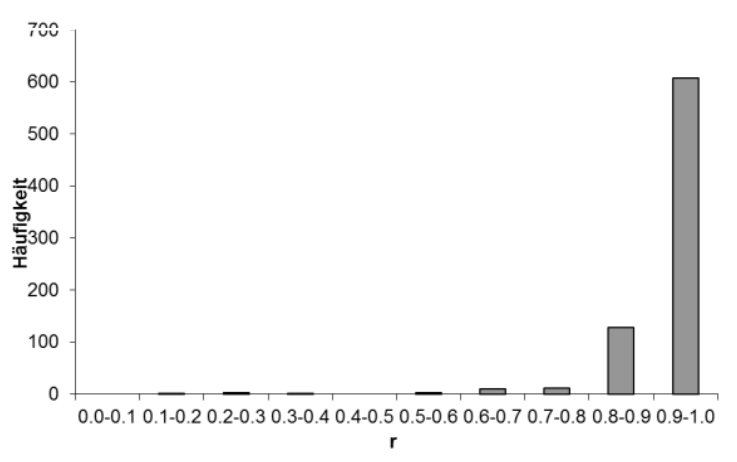

(b)

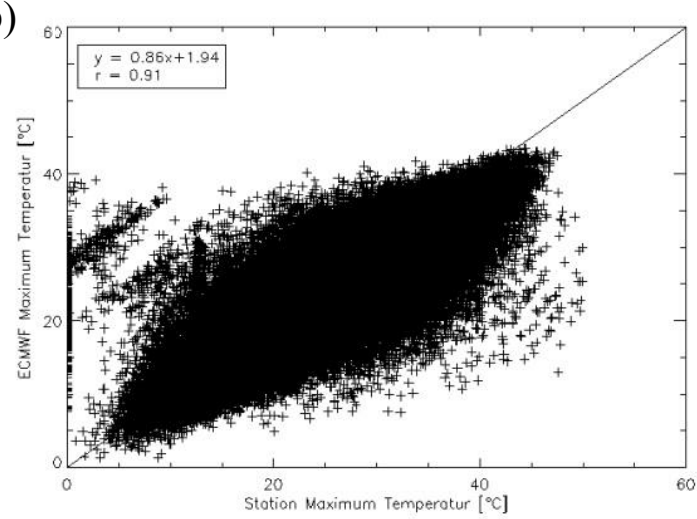

Abbildung 2.7: Verteilung der Korrelationskoeffizienten der einzelnen ARC-Messstationen (a) und das Streudiagramm aller Messwerte (b) für das Tagesmaximum der Temperatur. 
Die gute Übereinstimmung wird auch durch die Streudiagramme aller Messwerte (Abbildung 2.6b und Abbildung 2.7b) bestätigt. Mit 0.89 für $T_{\min }$ und 0.91 für $T_{\max }$ zählt die Temperatur zu den Eingangsdaten mit den höchsten Korrelationen bezüglich der Stationsdaten des ARC. Die Häufungen der Messwerte von $T_{\max }$ an der Stelle $0^{\circ} \mathrm{C}$ und im Bereich $0^{\circ}$ bis etwa $10^{\circ} \mathrm{C}$ werden auf Messfehler zurückgeführt, die nicht erkannt und eliminiert wurden. Im Hinblick auf die gute Korrelation der Daten können die Anzahl und die Auswirkung dieser Ausreißer allerdings vernachlässigt werden.

\subsubsection{Die Windgeschwindigkeit}

Da mit höherer Auflösung der turbulente Austausch, im Modell über die aerodynamische Leitfähigkeit berücksichtigt, an Bedeutung gewinnt, wird in der aktuellen Version anstatt eines konstanten Wertes der Windgeschwindigkeit von $u=3$, der Datensatz des ECMWF verwendet. Die Windgeschwindigkeit wird vier Mal täglich bezogen (4:00, 10:00, 16:00 und 22:00 Uhr) mit den Komponenten in Ost-West-, $\vec{u}_{x}$, und Nord-Süd-Richtung, $\vec{u}_{y}$. Daraus wird der Betrag der Windgeschwindigkeit $|u|=\sqrt{\vec{u}_{x}^{2}+\vec{u}_{y}^{2}}$ bestimmt, der jeweils zu den vier Zeitpunkten in das Modell zur Berechnung der aerodynamischen Leitfähigkeit $G_{a}$ (Gleichung (2.25)) eingeht.

(a)

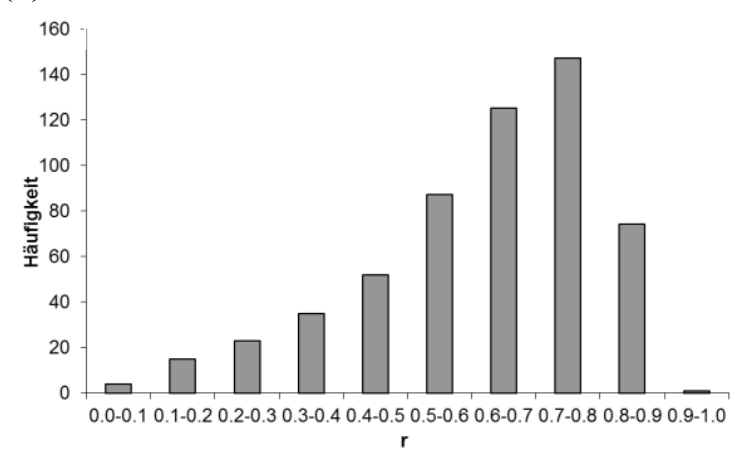

(b)

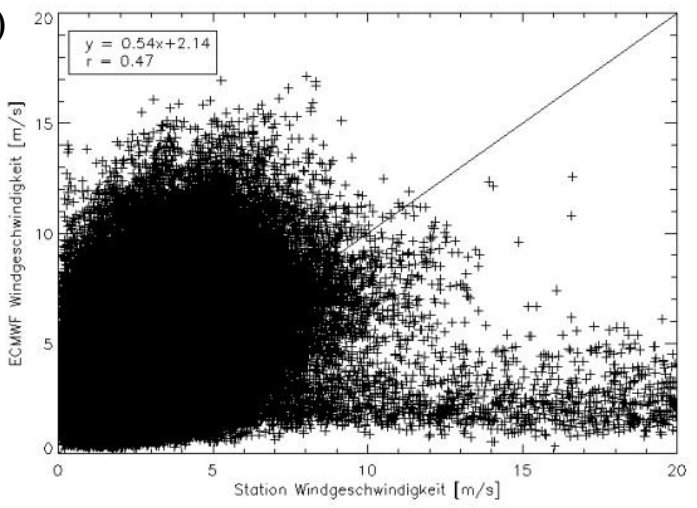

Abbildung 2.8: Verteilung der Korrelationskoeffizienten der einzelnen ARC-Messstationen (a) und das Streudiagramm aller Messwerte (b) für die Windgeschwindigkeit.

Abbildung 2.8a zeigt die Häufigkeitsverteilung der Korrelationswerte der 563 Messstationen, die Daten über die Windgeschwindigkeit liefern konnten. Zwar liegt das Maximum bei einem $r$ zwischen 0.7 und 0.8, fällt dann aber zu höheren Werten hin stark ab. Die meisten Stationen verteilen sich demnach auf Korrelationen unter 0.7. Dies wird auch im Streudiagramm (Abbildung 2.8b) deutlich, in dem außer einer Punktwolke kaum etwas zu 
erkennen ist. Daraus ergibt sich ein niedriger Korrelationsfaktor von 0.47 für alle Vergleichswerte. Speziell bei höheren Windgeschwindigkeiten scheinen die Messungen bzw. Berechnungen der ECMWF zu versagen und generell zu niedrige Werte zu erzeugen.

\subsubsection{Die photosynthetisch aktive Strahlung}

Schließlich wird die photosynthetisch aktive Strahlung (PAR) nach dem Ansatz von Burridge und Gadd (1974) aus den drei dominanten Wolkenschichten (hoch, mittel und tief) berechnet. Als Modellinput wird das Verhältnis von aktueller zu potentieller PAR benötigt, das sich aus dem Skalierungsfaktor des Bewölkungsgrades bestimmt. Die potentielle Strahlung, die bei unbewölktem Himmel an der Erdoberfläche zur Verfügung stehen würde, wird zunächst nach dem Ansatz von Weiss und Norman (1985) aus der Solarkonstante $S_{0}=1360 \mathrm{Wm}^{-2}$, dem Abstand zwischen Sonne und Erde (Paltridge und Platt, 1976)

$$
\begin{array}{rl}
r_{*}^{-2}=1 & .00011+0.034221 \cos \left(\alpha_{0}\right)+0.0128 \sin \left(\alpha_{0}\right)+ \\
& +0.000719 \cos \left(2 \alpha_{0}\right)+0.000077 \sin \left(2 \alpha_{0}\right)
\end{array}
$$

und dem Sonnenstand $\mu$

$$
\mu=\sin (\phi) \sin (\delta)-\cos (\phi) \cos (\delta) \cos [(\pi t / 12)-\lambda]
$$

berechnet. Dabei ist $\alpha_{0}=2 \pi(d-1) / 365$ mit dem julianischen Tag $d, \phi$ die geographische Breite, $\delta=-23.4(\pi / 180) \cos (2 \pi(d+10) / 365)$ die Position der Sonne, $t$ der aktuelle Zeitschritt und $\lambda$ die geographische Länge. Die potentielle PAR ergibt sich schließlich zu

$$
R_{P A R}=0.48 S_{0} r_{*}^{-2} \mu\left(0.4+0.6 e^{-\frac{0.185}{\mu} \frac{p}{p_{0}}}\right)
$$

Der Anteil, der bei Bewölkung an der Erdoberfläche ankommt, wird nach dem Ansatz von Stull (1988) mit Hilfe des Bewölkungsgrades bestimmt, der in tiefe $\left(\sigma_{c, l}\right)$, mittlere $\left(\sigma_{c, m}\right)$ und hohe $\left(\sigma_{c, h}\right)$ Wolkenschichten aufgeteilt wird. Der Transmissionskoeffizient ergibt sich $\mathrm{zu}$

$$
r_{P A R}=(0.6+0.2) \sin (\delta)\left(1-0.4 \sigma_{c, h}\right)\left(1-0.7 \sigma_{c, m}\right)\left(1-0.4 \sigma_{c, l}\right) .
$$


Aus dem Tagesmittel wird schließlich ein idealisierter Tagesgang der PAR berechnet. Diese Herleitung erzielte bessere Ergebnisse als die direkte Verwendung der Vorhersagedaten der Strahlung des ECMWF (Wisskirchen, 2005). Die globale Einstrahlung selbst wird für jedes Pixel in stündlichen Zeitschritten bestimmt.

575 Stationen standen für den Vergleich der photosynthetisch nutzbaren Einstrahlung zur Verfügung. Verglichen wurden zunächst die täglichen Werte der Tagessumme an einfallender Strahlung. Daraus ergeben sich für die meisten Stationen Korrelationswerte mit den ECMWF-Daten im Bereich von 0.7 bis 0.9 (Abbildung 2.9a).

(a)

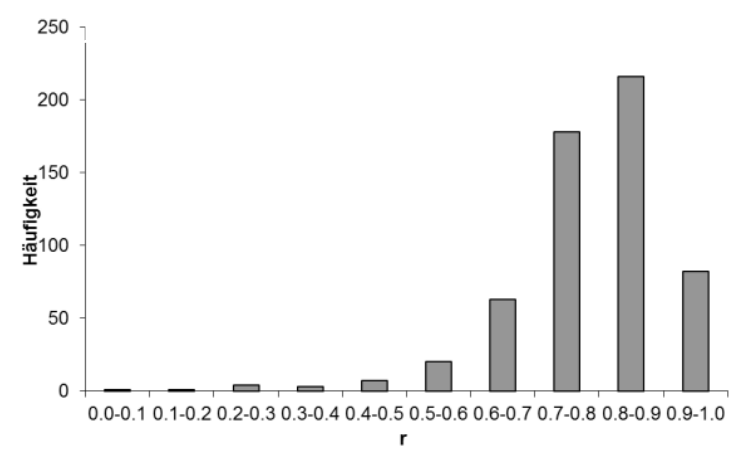

(b)

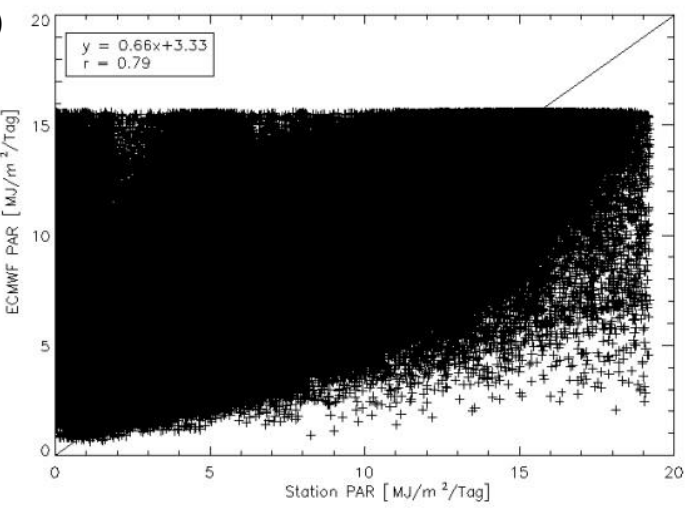

Abbildung 2.9: Verteilung der Korrelationskoeffizienten der einzelnen ARC-Messstationen (a) und das Streudiagramm aller Messwerte (b) für die PAR.

Ein Vergleich der Tageswerte aller Stationen mit den ECMWF-Werten ergibt ein $r$ von 0.79. Wie in Abbildung 2.9b zu sehen, ist dennoch bei der hohen Zahl der Werte die Streuung so hoch, dass dieser Zusammenhang nicht erkennbar wird. Hier soll für eine bessere Veranschaulichung ergänzend das Streudiagramm der Monatssummen gezeigt werden (Abbildung 2.10). Auch hier ergibt sich erwartungsgemäß ein höherer Korrelationskoeffizient von 0.9, aber auch die in den Tageswerten nicht ersichtliche Unterschätzung der PAR des ECMWF bezüglich der Stationsdaten wird hier ansatzweise deutlich. Außerdem ist in Abbildung 2.9b die unterschiedliche Ableitung der PAR aus den Messgrößen an den Maximalwerten zu erkennen. Im Falle der Stationsmessungen wird der für die Vegetation relevante Anteil der einfallenden Solarstrahlung aus der gemessenen Gesamteinstrahlung über den konstanten Faktor 0.48 bestimmt. Damit ergibt sich ein Maximum der PAR von etwa $19 \mathrm{MJ} \mathrm{m}^{-2} \mathrm{Tag}^{-1}$ Aus dem oben vorgestellten Ansatz zur Ableitung der potentiellen PAR bei wolkenlosen Bedingungen ergibt sich eine maximale Tagessumme von etwa 15.6 $\mathrm{MJ} \mathrm{m}^{-2} \mathrm{Tag}^{-1}$. 


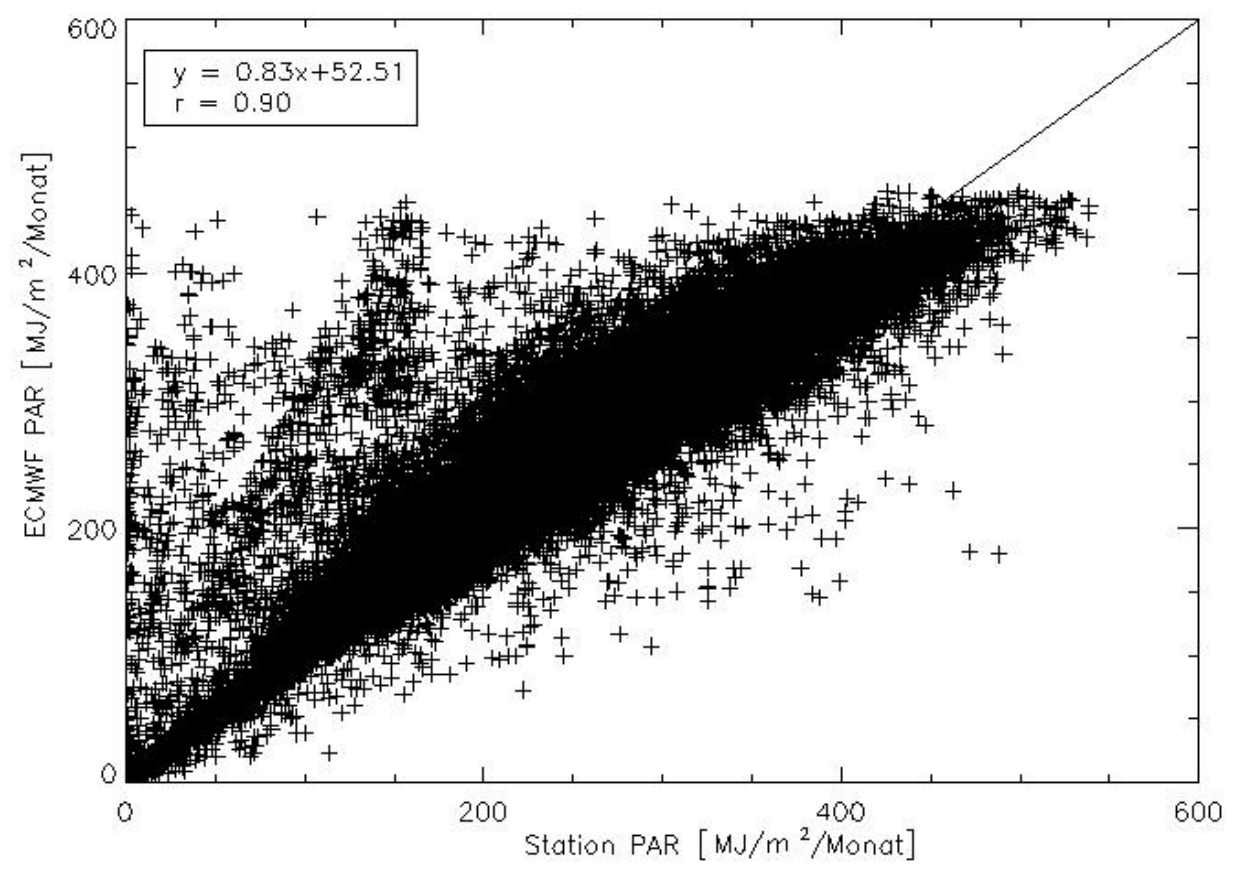

Abbildung 2.10: Streudiagramm der Monatssummen der PAR aller ARC Messstationen gegen die Werte des ECMWF.

Zusammenfassend geht aus dem Vergleich mit den Stationsdaten des ARC hervor, dass die Parameter des meteorologischen Antriebs aus der Datenbank des ECMWF nicht durchgängig von guter Qualität sind. Sehr gute Korrelationen konnten für die ECMWF-Datenreihen der Temperatur und der PAR bestimmt werden. Besonders wenig Übereinstimmung wurde dagegen für die Windgeschwindigkeit und den Niederschlag ermittelt. Die Sensitivität des Modells auf die Eingangsparameter wird in Kapitel 3 geprüft. Dadurch kann abschließend eingeschätzt werden, ob eine schlechte Güte der Eingangsdaten maßgeblich die Genauigkeit der Modellergebnisse beeinflusst.

\subsubsection{Der Blattflächenindex}

Einen der wichtigsten Eingangsparameter stellt der Blattflächenindex (im Folgenden LAI für Leaf Area Index) dar. Dieser wird allgemein als die einseitige Blattoberfläche des Vegetationsbestandes je Bestandsfläche definiert, und wird meist mit der Einheit $\mathrm{m}^{2} \mathrm{~m}^{-2}$ versehen. Der Jahresverlauf des LAI liefert somit Auskunft über den Blätterbewuchs, vor allem die saisonale Entwicklung bei sommergrünen Pflanzen. Für immergrüne Vegetation ist der LAI hauptsächlich Indikator für die Dichte und den Zustand des Bewuchses. 
BETHY/DLR verwendet ein LAI-Produkt, das aus Messungen des VEGETATION Sensors der SPOT (Satellite Pour l'Observation de la Terre) Satelliten generiert wird. Für den Zeitraum 1999 - 2007 wird dieser Datensatz von POSTEL (Pôle d'observation des surfaces continentales par télédétection), einem Projekt des französischen Forschungsnetzwerkes Medias France, produziert. Den Zeitraum von September 2009 bis heute prozessiert die belgische Firma VITO (vision on technology) im Rahmen des Projektes geoland2. Beide Produkte werden unter Verwendung des gleichen Prozessors - CYCLOPES (Carbon cycle and change in land observational products from an ensemble of satellites) - generiert (Baret et al., 2007). Die Daten werden als $10^{\circ} \times 10^{\circ}$ Kacheln in der Lat-Lon-Projektion WGS84 angeboten, angegeben mit H[0-35]V[0-17] (siehe Abbildung 2.11). Dabei bezeichnet $\mathrm{H}$ die horizontale (also 36 Kacheln) und $\mathrm{V}$ die vertikale Nummerierung (also 18 Kacheln). Jede Kachel enthält 1120 x 1120 Pixel, wodurch sich die räumliche Auflösung zu $1 / 112^{\circ}$ (etwa $1 \mathrm{~km}$ in Äquatornähe) ergibt.

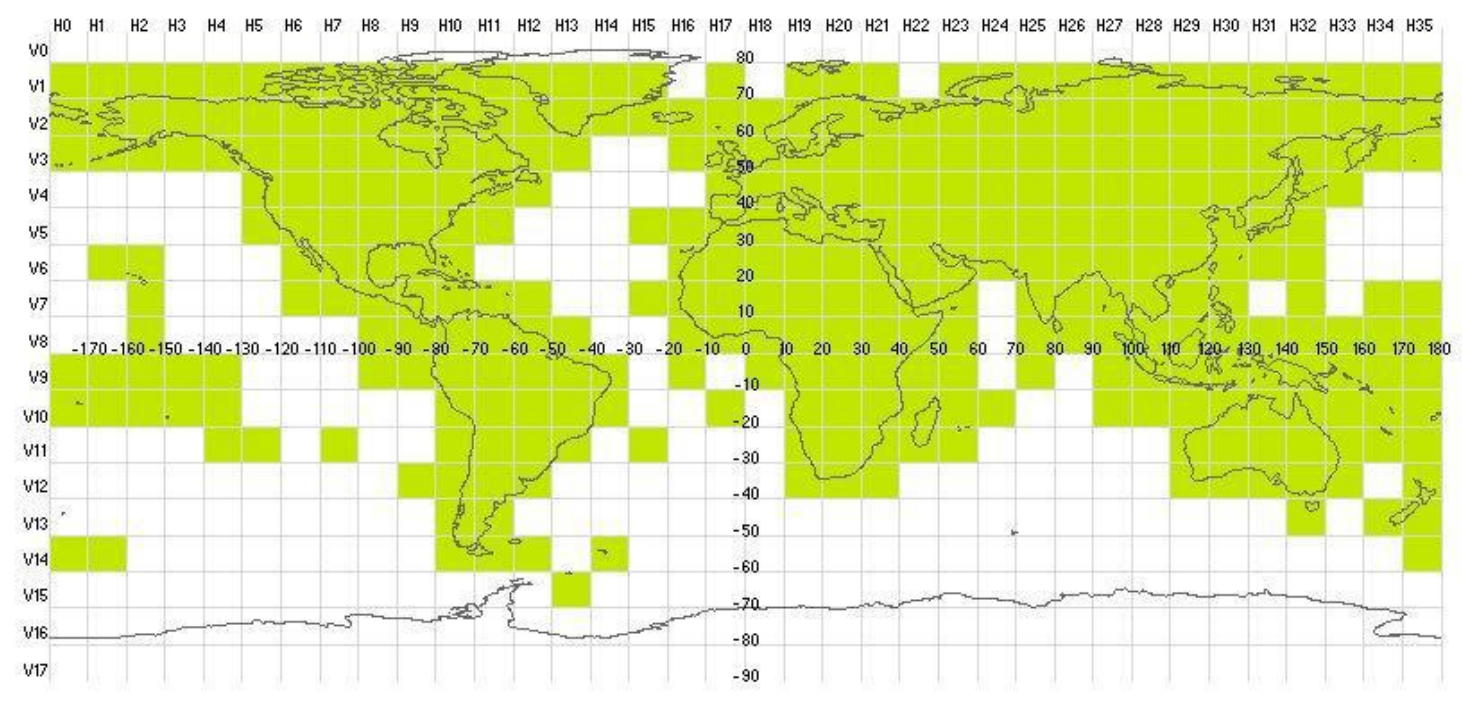

Abbildung 2.11. Globale Kachelung des SPOT-VGT LAI Produkts. Nur für grün markierte Kacheln wurden LAI Werte prozessiert. (Quelle: geoland2, Baret et al., 2010)

Die zeitliche Auflösung beträgt 10 Tage, wobei für die Zusammensetzung des Komposits ein Fenster von 30 Tagen verwendet wird. Nach Wolken- und Schneeerkennung, Atmosphärenkorrektur und BRDF-Normalisierung (Bidirectional Reflectance Distribution Function) werden aus den aufbereiteten SPOT-VGT-Daten die CYCLOPES-LAI-Werte berechnet. Dazu wird ein neuronales Netz, trainiert über ein Strahlungstransportmodell, verwendet. Für eine erfolgreiche Zusammensetzung werden schließlich mindestens zwei gültige Aufnahmen benötigt, deren Wert innerhalb des Intervalls $[0,8]$ liegen muss. Ist eine der Voraussetzungen nicht gegeben, wird für diesen Zeitschritt kein Wert angegeben (Baret 
et al., 2007). Daher enthalten die generierten Zeitreihen Lücken, vor allem durch gebietsweise hohes Wolkenaufkommen, aber auch durch Sensorprobleme oder Fehler bei der Datenabfrage. Große Schattenfelder, bedingt durch niedrige Sonnenwinkel während der Aufnahme oder ein erhöhtes Rauschen durch den verlängerten optischen Weg durch die AtmoAtmosphäre, sind ebenfalls ein Grund für auftretende Datenlücken.

Für die Modellierung mit BETHY/DLR werden allerdings generell kontinuierliche Datensätze benötigt, so dass für den Fall des LAI ein gesonderter Schritt der Vorprozessierung nötig ist. Hierzu wird die Methode der Harmonischen Analyse (Bittner et al., 1994) verwendet, die am DLR bereits zur Prozessierung von satellitengestützten Messungen von Ozonsäulen verwendet wird (Bittner et al., 1998; Meisner et al., 1999, Erbertseder et al., 2006). Da diese Methode allerdings nicht für die Prozessierung großer Datenlücken konzipiert wurde, mussten einige Änderungen vorgenommen werden. Unter Verwendung einer mittleren Phänologie werden zunächst größere Lücken von mehr als fünf Datenpunkten aufgefüllt, noch vorhandene Ausreißer in den Daten werden ermittelt und eliminiert. Abschließend wird eine Modifikation der Harmonischen Analyse (HA) verwendet, um die Zeitreihen zu glätten und kleinere Lücken aufzufüllen. Die Funktionsweise der HA und die Erweiterungen zur Anwendung auf den LAI werden im Folgenden erläutert.

Die Idee, die HA auf die Zeitreihen des LAI anzuwenden stützt sich auf den Gedanken, dass die saisonale Variabilität des LAI als eine Überlagerung von Schwingungen mit verschiedenen Perioden beschrieben werden kann. Die erste harmonische Schwingung spiegelt beispielsweise für gemäßigte Regionen den Jahresgang der mittleren Temperatur wieder. Für subtropische Vegetation wäre hier die Regenzeit der treibende Faktor. Weitere Schwingungen können die Bewirtschaftung der Vegetation oder kurzfristige Schwankungen in den meteorologischen Gegebenheiten wiedergeben. Eben diese Überlagerung einzelner Schwingungen zu einer Zeitreihe ist das Grundkonzept der HA (siehe Abbildung 2.12). Jede Schwingung ist definiert über eine Amplitude $A_{i}$, eine Phase $\varphi_{i}$ und eine Frequenz $f_{i}$, wobei $i$ von 1 bis $n$ reicht, wenn $n$ die Anzahl der harmonischen Schwingungen ist. Durch die sukzessive Dekonvolution des Leistungsdichtespektrums wird nach und nach der jeweils höchste Beitrag (also die Schwingung mit der größten Amplitude) abgezogen und jeweils das neue Spektrum berechnet. Die Varianz der Zeitserie kann dadurch weiter reduziert werden, dass zu jedem weiteren Schritt die Parameter der aktuellen sowie der vorherigen Sinusschwingungen nochmals gleichzeitig variiert werden. Wenn also die zweite Schwingung angepasst werden soll, sind Amplitude, Phase und Frequenz der ersten Schwingung wieder freie Parameter. Für die Lösung des linearen Gleichungssystems werden das Newton-Raphson- (z.B. Ortega und Rheinbold, 1970) und das Cholesky-Verfahren (Cholesky 1924) verwendet. 


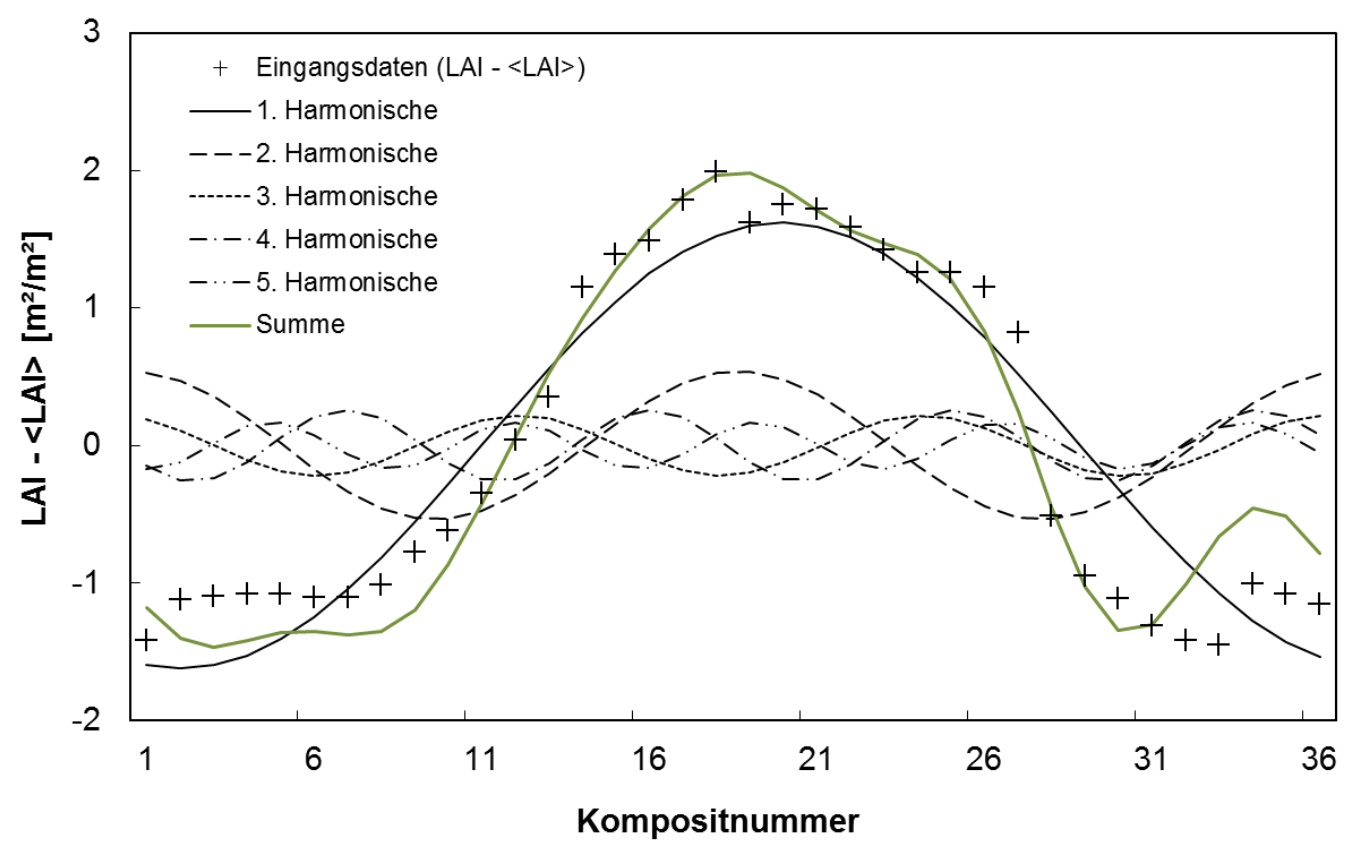

Abbildung 2.12. Zerlegung einer Zeitreihe durch die Harmonische Analyse in fünf harmonische Einzelschwingungen.

Eine Linearkombination von Sinusschwingungen mit zugehörigen Amplituden, Phasen und Frequenzen wird ermittelt, deren Summe eine beste Anpassung an die Originaldaten darstellt:

$$
y(t)=\sum_{i=1}^{n} A_{i} \cdot \sin \left(\frac{2 \pi}{\tau_{i}} t+\varphi_{i}\right)
$$

Hier entspricht $y(t)$ dem LAI-Wert, $A_{i}$ der Amplitude, $\tau_{i}$ der Periode und $\varphi_{i}$ der Phase der $i$ ten Schwingung. Dieses Vorgehen wird für jeden Zeitschritt der Zeitreihe durchgeführt. Daraus ergibt sich eine Reihe von Wellenlängen die, bezüglich der Länge der Zeitreihe, in äquidistanten Abschnitten verteilt sind. Innerhalb dieser Reihe werden die optimale Amplitude und Phase bestimmt, um die Schwingung anzupassen. Von dem entstehenden Periodogramm kann die Varianz der dominanten spektralen Charakteristika gefolgert werden. Daraus wird die Wellenlänge, die die Variabilität der gesamten Zeitserie am besten beschreibt, ausgewählt und bezüglich Amplitude und Phase mit Hilfe der Messwerte optimiert. Dies wird für das Erkennen weiterer Schwingungen wiederholt, wodurch mit jedem weiteren Schritt vorher gefundene Schwingungen optimiert werden.

Um die Notwendigkeit einer Anpassung der ursprünglichen Formulierung der HA an die Erfordernisse einer Analyse von LAI-Zeitserien aufzuzeigen, ist in Abbildung 2.13 das 
Ergebnis für ein Pixel mit Datenlücken dargestellt. Das Pixel ist klassifiziert als sommergrüner Laubwald in Massachusetts, USA, für das Jahr 2003. Hier treten drei Lücken auf, von denen die Erste sieben Datenpunkte, also einen Zeitraum von siebzig Tagen, umfasst, die anderen beiden jeweils einen Datenpunkt, also zehn Tage. Die durchgezogene Linie zeigt das Ergebnis der HA in ihrer ursprünglichen Version. Der Verlauf der Originalmessungen konnte hier nicht nachvollzogen werden. Es werden zwar zwei Maxima während der vegetativ aktiven Phase in der Mitte des Jahres dargestellt, die auch in den Originaldaten gefunden werden können, allerdings liegen die Werte um etwa $2 \mathrm{~m}^{2} \mathrm{~m}^{-2}$ zu niedrig.

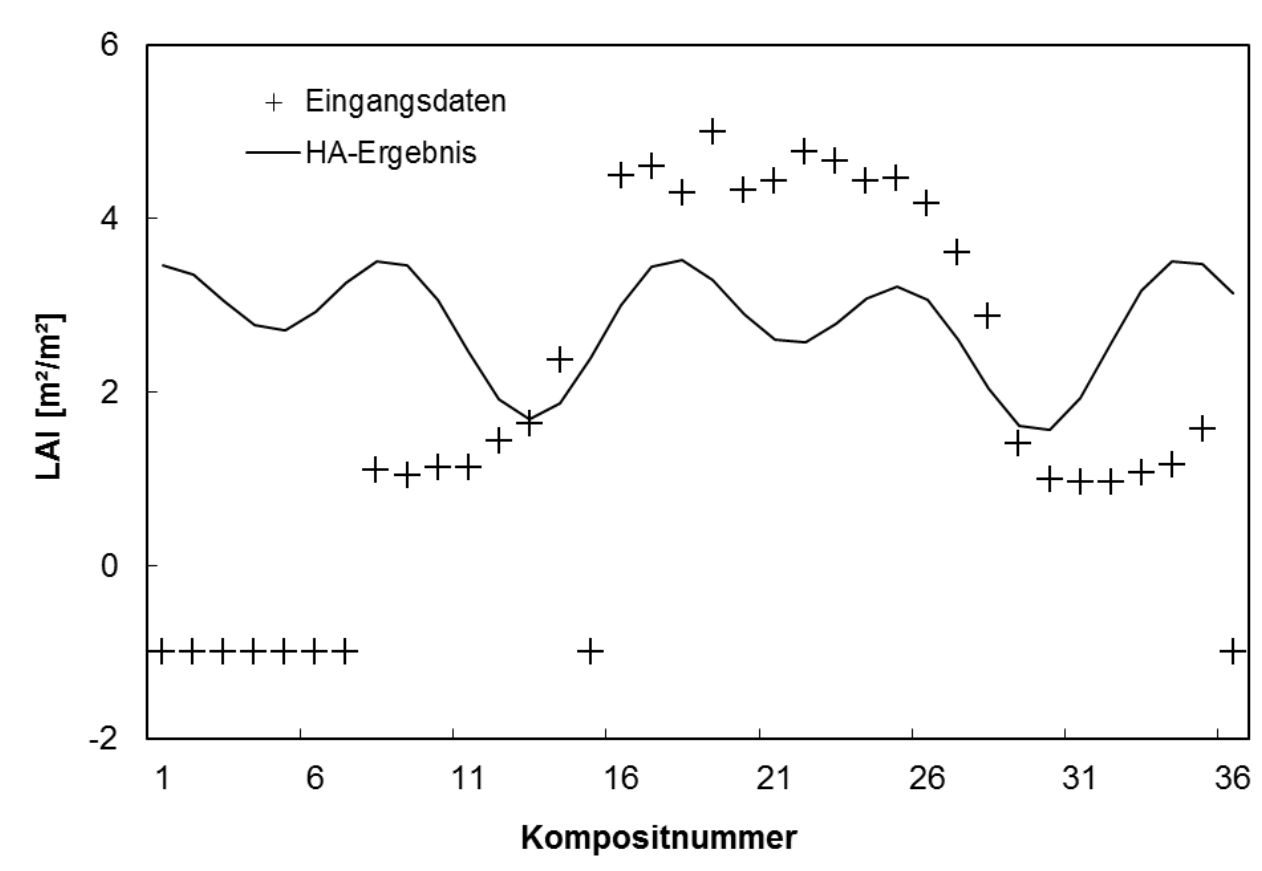

Abbildung 2.13. Ergebnis der ursprünglichen Version der HA angewandt auf eine LAI-Zeitserie mit Datenlücken (dargestellt mit Wert -1). Die Zeitserie zeigt ein als sommergrüner Laubwald klassifiziertes Pixel in Massachusetts, USA, für das Jahr 2003.

Der Verlauf zu Beginn und Ende des Jahres weicht generell von dem der vorhandenen Originaldaten ab. Dies begründet sich hauptsächlich darin, dass die HA in ihrer ursprünglichen Version nicht dafür ausgelegt war, Zeitreihen mit sehr großen Datenlücken zu interpolieren. Es hat sich gezeigt, dass kleinere Lücken von bis zu fünf Datenpunkten kein Problem darstellen, sich für längere Perioden von fehlenden Daten allerdings unphysiologische Resultate ergeben. Längere Datenlücken entstehen entweder durch längere Wolkenbedeckung einer Region oder dadurch, dass die Satellitendaten ab einem solaren Zenithwinkel von weniger als $60^{\circ}$ nicht mehr prozessiert werden. In Regionen in Äquatornähe oder in den Tropen kann es vorkommen, dass eine fast permanente Wolkenbedeckung die Erzeugung einer LAI-Zeitreihe komplett verhindert. Die Anzahl an fehlenden Daten und deren Perio- 
denlänge ist also von geographischer Breite und Jahreszeit abhängig. Daher wurde das Verfahren eingeführt, zusammenhängende Lücken von mehr als vier Zeitschritten, mit LAIWerten einer mittleren Phänologie der betrachteten Landbedeckungsklasse zu füllen. Diese mittlere Phänologie wird jährlich für jede $10^{\circ}$ x $10^{\circ}$ Kachel für jeden Landbedeckungstyp berechnet. Dazu werden alle zugehörigen LAI-Werte für jeden Zeitschritt einzeln gemittelt. Die Einteilung der Landbedeckungstypen erfolgt über die Verwendung der globalen Landbedeckungsklassifizierung für das Jahr 2000 (Global Land Cover GLC2000), durch die 24 verschiedene Typen unterschieden werden (Bartholomé, 2002; Fritz et al., 2003). Die GLC2000 ist ebenfalls in dem SPOT-VGT-LAI-Produkt enthalten und wird in Kapitel 2.4.3 näher beschrieben.

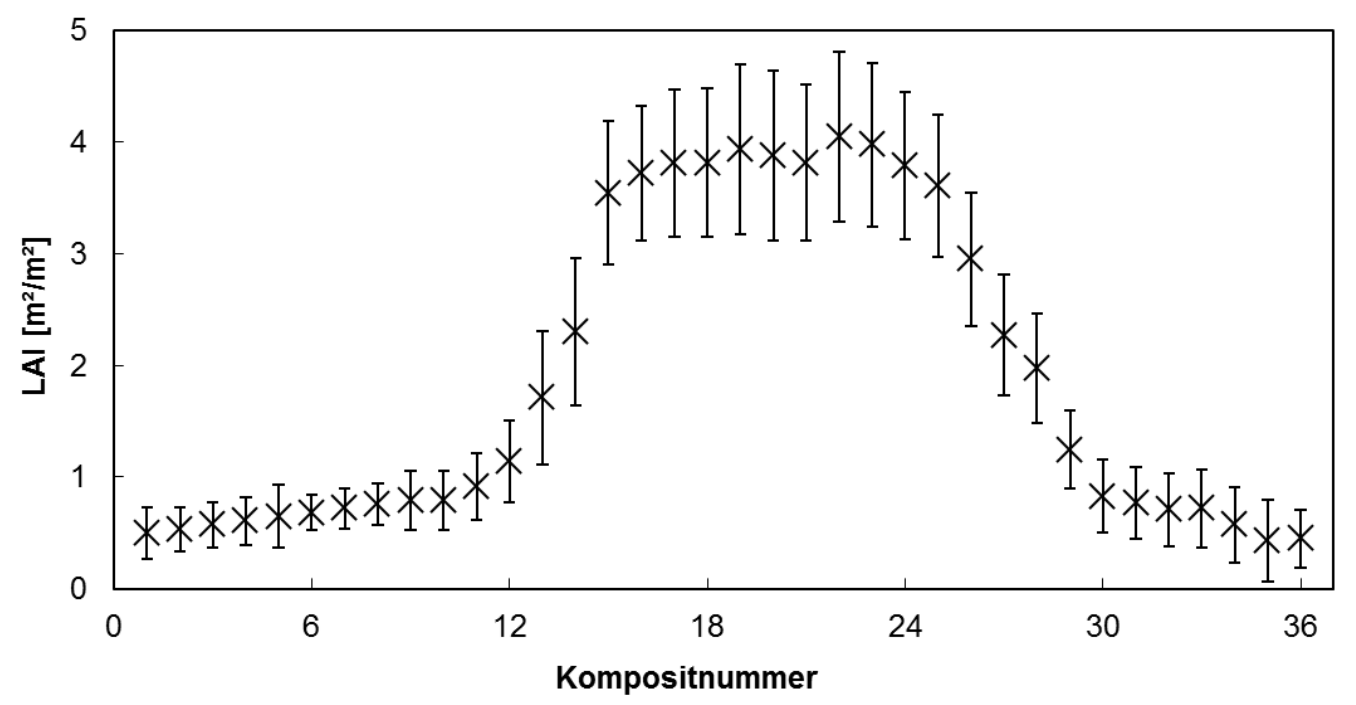

Abbildung 2.14. Mittlere Phänologie der Vegetationsklasse ,sommergrüner Laubwald' für das Jahr 2003 in der Kachel ,H10V4'. Die Fehlerbalken geben die Standardabweichung der einzelnen Werte an.

Dieses Verfahren beruht auf der Annahme, dass sich die Phänologien der einzelnen Bedeckungsklassen innerhalb einer $10^{\circ}$ x $10^{\circ}$ Kachel für alle Pixel ähnlich sind. Eine solche mittlere Phänologie für eine sommergrüne Laubwaldvegetation zeigt Abbildung 2.14, berechnet für die Kachel 'H10V4' und das Jahr 2003. Die Kachel zeigt den Nordosten der Vereinigten Staaten und südöstliche Teile Kanadas (obere linke Ecke: $80 \mathrm{~W} 50 \mathrm{~N}$; untere rechte Ecke: 70W 40N). Diese Zeitreihe der mittleren Phänologie wird in der endgültigen Version der vorgestellten Methode zum Füllen der größeren Lücke der Zeitserie in Abbildung 2.13 verwendet.

Ein weiterer Schritt zur Aufbereitung der Eingangsdaten ergibt sich aus der Notwendigkeit bei der pixelbasierten Identifizierung der harmonischen Schwingungen zwischen natürli- 
chen und durch Störungen erzeugten spektralen Strukturen zu unterscheiden (Bittner et al., 1994). Ausreißer können das Ergebnis der Zeitreihenanalyse erheblich beeinflussen. Obwohl die Prozesskette für die Erstellung des SPOT-VGT-LAI-Produkts bereits einen Algorithmus zur Erkennung von Ausreißern enthält, können in dem Produkt dennoch physiologisch fragwürdige Sprünge zwischen den Zeitschritten beobachtet werden. Zur Erklärung müssen die verschiedenen Schritte in der Vorprozessierung zum Training des neuronalen Netzes, das verwendet wird, um den LAI abzuleiten, betrachtet werden (Baret et al., 2007). Nach der Wolkenerkennung und der atmosphärischen Korrektur wird das Roujean'sche BRDF-Model (Roujean et al., 1992) verwendet, um die Kronendach-Reflexionen der drei VEGETATION-Bänder (B2, B3 und MIR) auf den Nadir-Beobachtungswinkel zu normalisieren. Für die BRDF-Korrektur werden wolkenfreie Beobachtungen über ein Fenster von \pm 15 Tagen benötigt. In einem iterativen Prozess werden Ausreißer in den Reflexionen erkannt und entfernt, die durch Wolkenbedeckung oder unsaubere atmosphärische Korrektur verursacht werden. Schließlich werden die Mediane für alle drei BRDF-korrigierten Bänder und des Sonnenzenithwinkels berechnet. Die normalisierten Reflexionen werden als Eingangsdaten für das neuronale Netz verwendet. Dass dennoch Ausreißer in den LAIZeitserien beobachtet werden, könnte damit erklärt werden, dass diese Inputvariablen ausserhalb des Variablenraumes liegen, der zum Training des neuronalen Netzes verwendet wurde. Diese Abweichung kann auftreten, wenn Wolken, Nebel oder Schatten nur unzureichend erkannt wurden. Ebenso kann die Atmosphärenkorrektur aufgrund von hohen Aerosolanteilen, die nicht in der Aerosolklimatologie berücksichtigt werden, fehlerhaft sein.

Generell können Ausreißer in den LAI-Zeitserien als hochfrequenter Schwingungsanteil betrachtet werden, der die durch die HA gefundenen Amplituden und Phasen beeinflusst und dadurch größere Fehler verursacht. Ein Ausweg wäre, Ausreißer als Werte zu definieren, die außerhalb eines Wertebereiches von $\pm 3 \sigma$ um die mittlere Phänologie liegen, wobei $\sigma$ der Standardabweichung der mittleren Phänologie entspricht. Nach diesem Verfahren werden $0.3 \%$ aller Werte als Ausreißer erkannt, allerdings haben Tests gezeigt, dass dies nicht alle unphysiologischen Verläufe mit einschließt. Daher wurde ein weiterer Ansatz verfolgt und getestet. Dabei wird in einem ersten Schritt jede Lücke mit dem zugehörigen Wert der mittleren Phänologie gefüllt. In einem zweiten Schritt werden nacheinander jeweils drei aufeinanderfolgende Werte betrachtet. Als Ausreißer werden solche Werte definiert, deren Gradienten zu dem jeweils vorherigen und nachherigen Wert unterschiedliche Vorzeichen besitzen und der Wert mindestens eines der Gradienten größer ist als $2 \mathrm{~m}^{2} \mathrm{~m}^{-2}$ pro Zeitschritt.

$$
\operatorname{sgn}\left(\Lambda_{i}-\Lambda_{i-1}\right) \neq \operatorname{sgn}\left(\Lambda_{i+1}-\Lambda_{i}\right) \wedge\left(\left(\Lambda_{i}-\Lambda_{i-1}\right)>2 \vee\left(\Lambda_{i+1}-\Lambda_{i}\right)>2\right)
$$


Ein kontinuierlicher, steiler Anstieg oder Abfall des LAI wird nicht als Ausreißer erkannt. Sowohl eigene Tests als auch andere Arbeiten haben bestätigt (Verger et al., 2011), dass der Vorzeichenwechsel des Gradienten und ein Anstieg oder Abfall des LAI von mehr als $2 \mathrm{~m}^{2}$ $\mathrm{m}^{-2}$ pro Zeitschritt unrealistisch schnelle Wechsel im Verlauf des Pflanzenwachstums darstellen. Als Ausreißer erkannte Werte werden als , bad value' markiert und in der HA nicht betrachtet.

Nach der Identifizierung der Ausreißer und aller Datenlücken wird die Zeitreihe verdoppelt und hintereinander angeordnet. Dies hat den Vorteil, dass die Datenlücken zu Beginn und am Ende des Jahres nun in der Mitte der verdoppelten Zeitreihe als eine zusammenhängende Lücke analysiert werden können. Die größte Lücke umfasst im Falle der Datenreihen von Abbildung 2.13 nun acht Datenpunkte, zusammengesetzt aus sieben fehlenden Datenpunkten zu Beginn des Jahres und einem Datenpunkt am Ende des Jahres.

Eine wesentliche Modifikation der Methode der HA betrifft die Anzahl der Schwingungen $n$ und deren Periodendauer $\tau$. In der ursprünglichen Formulierung wurde eine obere und eine untere Periodendauer $\left(\tau_{u p}, \tau_{l o}\right)$ als Beschränkung für die erlaubte Periodendauer definiert. Aus diesem Intervall wurden dann die Periodendauern der Ausgleichskurve bestimmt, zusammen mit der Anzahl verwendeter Schwingungen für die Linearkombination der gewählten Sinuskurven (Formel (2.59)). Die Startwerte der zu wählenden Periodenlängen der $n$ Schwingungen wurden gleichmäßig zwischen $\tau_{u p}$ und $\tau_{l o}$ verteilt. Die $i$-te Periodenlänge war daher definiert als $\left(\tau_{u p}-\tau_{l o}\right) \cdot i / n$. Durch diese Verteilung der Periodenlängen wurden im Allgemeinen nur zwei Schwingungen gefunden, bevor die Ausstiegsbedingungen erfüllt waren. Zwei Schwingungen sind allerdings meist unzureichend, um die Charakteristika der zugrundeliegenden LAI-Zeitreihe zu beschreiben. Um diesen Nachteil aufzuheben, wurden zwei Änderungen vorgenommen. Zunächst wurden die Ausstiegsbedingungen für das Finden der harmonischen Schwingungen angepasst. Für den Ausstieg aus der Iteration zur Suche der besten Ausgleichskurve werden die Residuen der aktuellen Berechnung und der des vorherigen Schrittes verglichen. Die Differenz der Varianzen der Residuen wird durch den Faktor $\varepsilon$ kontrolliert. Ursprünglich war dieser Faktor auf einen konstanten Wert von 0.5 gesetzt und wurde nun auf 0.001 als Startwert geändert. Wenn dieser Schwellenwert nach vier Iterationen nicht erreicht wird, wird $\varepsilon$ jeweils verdoppelt, wobei allerdings der ursprüngliche Wert von 0.5 selten erreicht wird.

In einem weiteren Schritt wurden die Startwerte für die Periodenlängen nach Schönwiese (1985) neu angeordnet. Dadurch werden diese nun über die kürzeste Periodenlänge und die Anzahl an Schwingungen definiert. Die Verteilung ist damit nicht mehr äquidistant, sondern häuft sich zu kürzeren Periodenlängen hin. Die i-te Periode ist also definiert als $\tau_{l o} / i$. 
Im Vergleich zu der ursprünglichen Anordnung werden nun frühzeitig auch Schwingungen mit kurzen Periodenlängen zur Beschreibung der Zeitreihe mit einbezogen. Dieses Vorgehen hat zusätzlich den Vorteil, Rechenzeit einzusparen, nachdem die veränderte Formulierung der HA, vor allem durch die Verdoppelung der Zeitreihe, mehr Zeit in Anspruch nehmen wird.

Die Anzahl an harmonischen Schwingungen wird weiterhin durch ein Minimum von zwei Schwingungen und einer minimalen Periode von 60 Tagen (sechs Datenpunkten) beschränkt. Technisch sind bei der Methode der HA unendlich viele Schwingungen zugelassen, allerdings wird dies durch die Beschränkung der Periodenlänge eingeschränkt.

Zum Vergleich der durch die HA neu bestimmten LAI-Werte mit den Originaldaten, wird der Root Mean Square Error (RMSE) für jedes Pixel und Jahr berechnet.

$$
R M S E=\sqrt{\frac{\sum\left(\Lambda_{\text {input }}-\Lambda_{H A}\right)^{2}}{n}}
$$

Wobei $\Lambda_{\text {input }}$ die SPOT-VGT-Eingangsdaten bezeichnet, $\Lambda_{H A}$ die Ergebnisse der HA und $n$ die Anzahl der für die HA verwendeten Datenpunkte (also ohne Datenlücken). Um die RMSE-Werte untereinander besser vergleichen zu können hat sich die Verwendung des auf den Mittelwert $\bar{\Lambda}$ normalisierten RMSE (NRMSE) als hilfreich erwiesen:

$$
N R M S E=R M S E / \bar{\Lambda}
$$

Haben beispielsweise ein Graslandpixel mit einem mittleren LAI von 1 und ein Laubwaldpixel mit mittlerem LAI von 3 beide einen RMSE von 0.1 , bedeutet dies einen größeren relativen Fehler für das Graslandpixel. Dies wird nur durch die Darstellung über den NRMSE deutlich (NRMSE $=0.033$ für das Laubwaldpixel, NRMSE $=0.1$ für das Graslandpixel).

Um die Auswirkungen der beschriebenen Änderungen zu untersuchen, wurde die Methode nochmals auf die in Abbildung 2.13 gezeigte LAI-Zeitreihe angewandt. Das Ergebnis ist in Abbildung 2.15 zusammen mit der mittleren Phänologie für diesen Landbedeckungstyp dargestellt. Davon werden die ersten sieben Datenpunkte sowie der Letzte verwendet, um die fehlenden Werte zu ersetzen. Kleinere Lücken von weniger als fünf Datenpunkten können von der modifizierten HA ohne Auffüllen interpoliert werden. Durch die Modifikationen der HA kann der Verlauf der Pflanzenphänologie, vor allem in der photosynthetisch aktiven Phase im Sommer wesentlich besser dargestellt werden. Aber auch die inaktive 
Phase im Winter kann durch die veränderte Methodik besser dargestellt werden. Insgesamt erreicht das Ergebnis für dieses Pixel und dieses Jahr einen NRMSE von 0.1.

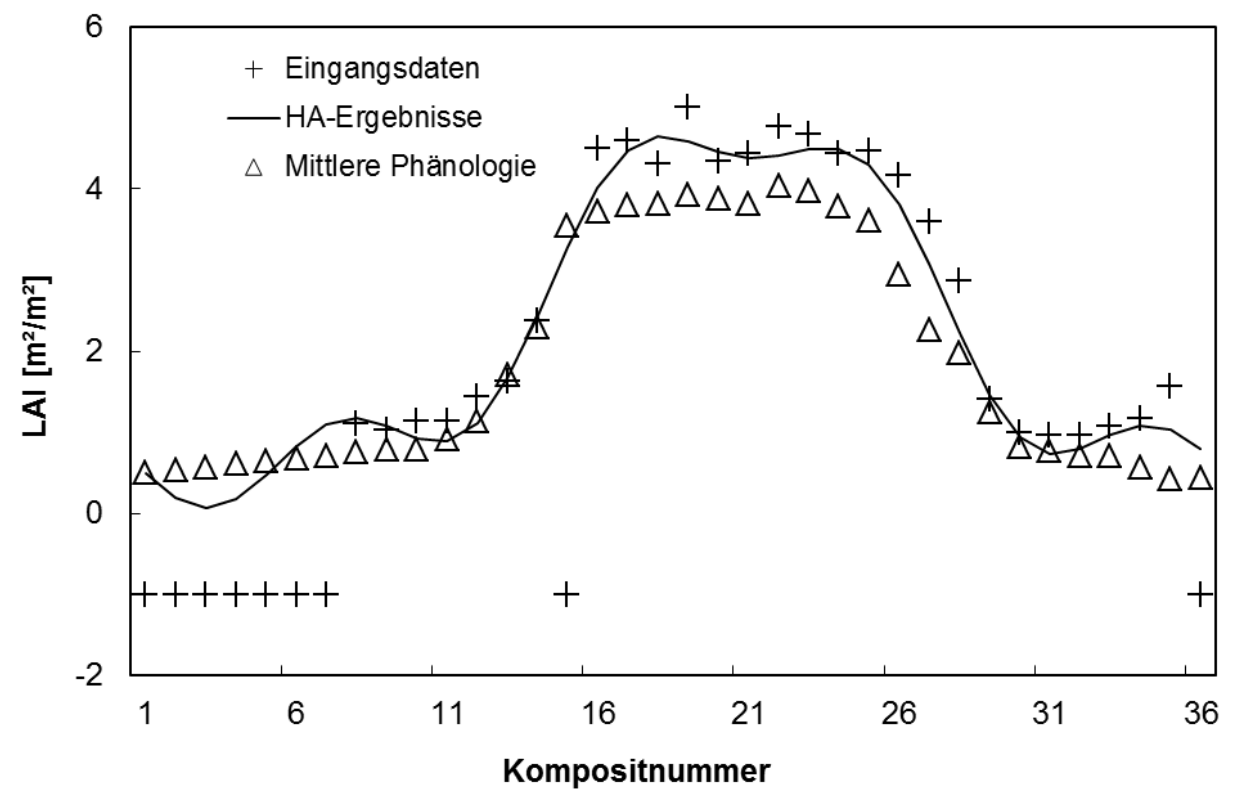

Abbildung 2.15. Ergebnis der modifizierten HA (durchgezogene Linie) angewandt auf die Originaldaten (Kreuze) des bereits in Abbildung 2.13 gezeigten Mischwaldpixels. Werte von -1 bezeichnen Datenlücken. Die Dreiecke stellen die mittlere Phänologie der zugehörigen Landbedeckungsklasse dar.

Um die Leistung der veränderten HA großflächig zu analysieren sind in Abbildung 2.16 und Abbildung 2.17 repräsentative, global verteilte Kacheln vor und nach der Anwendung der Analyse gezeigt. Die Kacheln sind H7V5 (Nordamerika, Zentrum: 105W 35N), H19V4 (Europa, Zentrum: 15E 45E), H29V4 (Asien, Zentrum: 115E 15N), H18V7 (Afrika, Zentrum: 5E 15N), H29V12 (Australien, Zentrum: 115E 35S) und H12V8 (Südamerika, 55W $5 N)$. Die Beispiele zeigen jeweils verschiedene Jahre und unterschiedliche Zeitschritte. Ein Blick auf die Ergebnisse macht deutlich, dass nicht alle Datenlücken über der Landoberfläche durch die HA aufgefüllt werden können (weiße Pixel). Für Pixel der Landoberfläche ergeben sich nach Baret et al. (2007) Datenlücken durch Wolken- oder Schneebedeckung. In Kachel H19V4 (Abbildung 2.16, mittlere Spalte) sind weiterhin Lücken in der Alpenregion zu finden. Diese sind entweder als blanker Boden klassifiziert oder fast das ganze Jahr über mit Schnee bedeckt. In H12V8 (Abbildung 2.17, rechte Spalte) stammen die Datenlücken daher, dass durch die Bewölkungshäufigkeit in Äquatornähe weniger als zehn Datenpunkte für eine Analyse zur Verfügung gestanden hätten. In einem solchen Fall wird keine HA durchgeführt. In den übrigen Regionen führt die Zeitreihenanalyse zu einer wesentlichen Verbesserung der räumlichen Abdeckung mit LAI-Daten. 

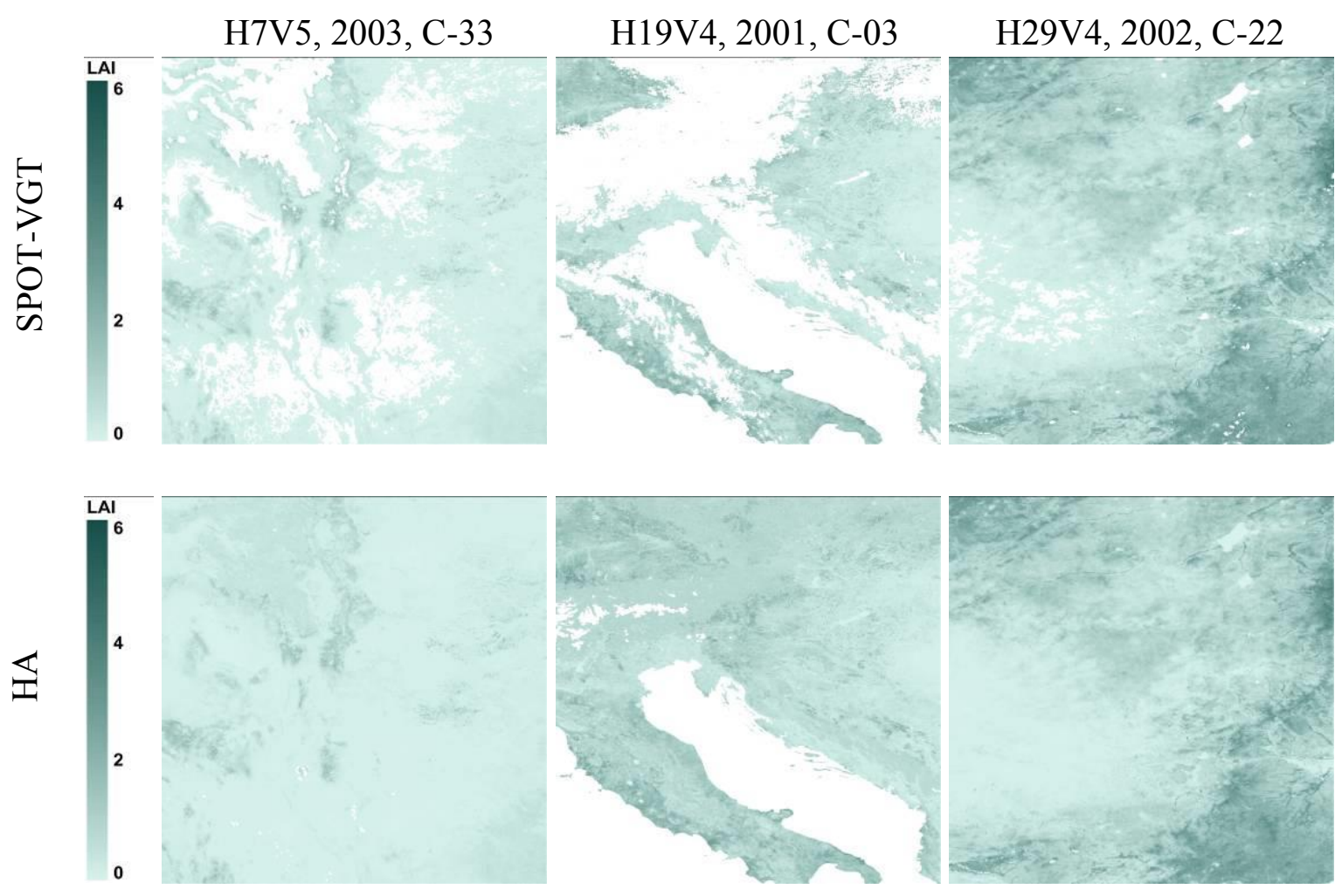

Abbildung 2.16. Vergleich von repräsentativen SPOT-VGT-Kacheln vor (erste Reihe, SPOT-VGT) und nach (zweite Reihe, HA) der Anwendung der Zeitreihenanalyse. Die Kacheln sind: Links, H7V5, Nordamerika; Mitte, H19V4, Europa; Rechts, H29V4, Asien. Die Kacheln und entsprechende Zeitpunkte sind oberhalb der Abbildung beschrieben. C-xx steht für das jeweilige Komposit des Jahres. Weiße Flächen stellen fehlende LAI-Werte bzw. Wasserpixel dar.

Obwohl der Algorithmus, abhängig von der Anzahl an Datenpunkten, $n$ harmonische Schwingungen zulässt, sind in der Regel nach den vorher beschriebenen Kriterien vier bis fünf Oszillationen ausreichend, um die LAI-Zeitserie durch die Überlagerung dieser Harmonischen darzustellen. Beispielsweise wurden für 24\% der in Kachel H19V4 prozessierten Pixel fünf Oszillationen benötigt, für 46\% vier Oszillationen, für 25\% drei Schwingungen und 5\% konnten mit der minimalen Anzahl von zwei Schwingungen beschrieben werden.

Zur weiteren Analyse sind in Abbildung 2.18 und Abbildung 2.19 in der obersten Reihe jeweils die absolute Differenz zwischen den SPOT-VGT-Daten und den HA-Ergebnissen, in der mittleren Reihe der NRMSE und in der unteren Reihe der Anteil an aufgefüllten Werten je Pixel gezeigt. Die Differenz wurde nur für Pixel berechnet, für die auch ein originaler SPOT-VGT-Wert vorhanden war. Für die meisten Regionen ist diese Abweichung nicht größer als $0.5 \mathrm{~m}^{2} \mathrm{~m}^{-2}$ mit Maximalwerten von etwa $\pm 1 \mathrm{~m}^{2} \mathrm{~m}^{-2}$. Die höchsten Unterschiede sind für immergrüne Laubwälder an der Südwest-Küste Australiens (Kachel H29V12 in Abbildung 2.19) zu sehen. 

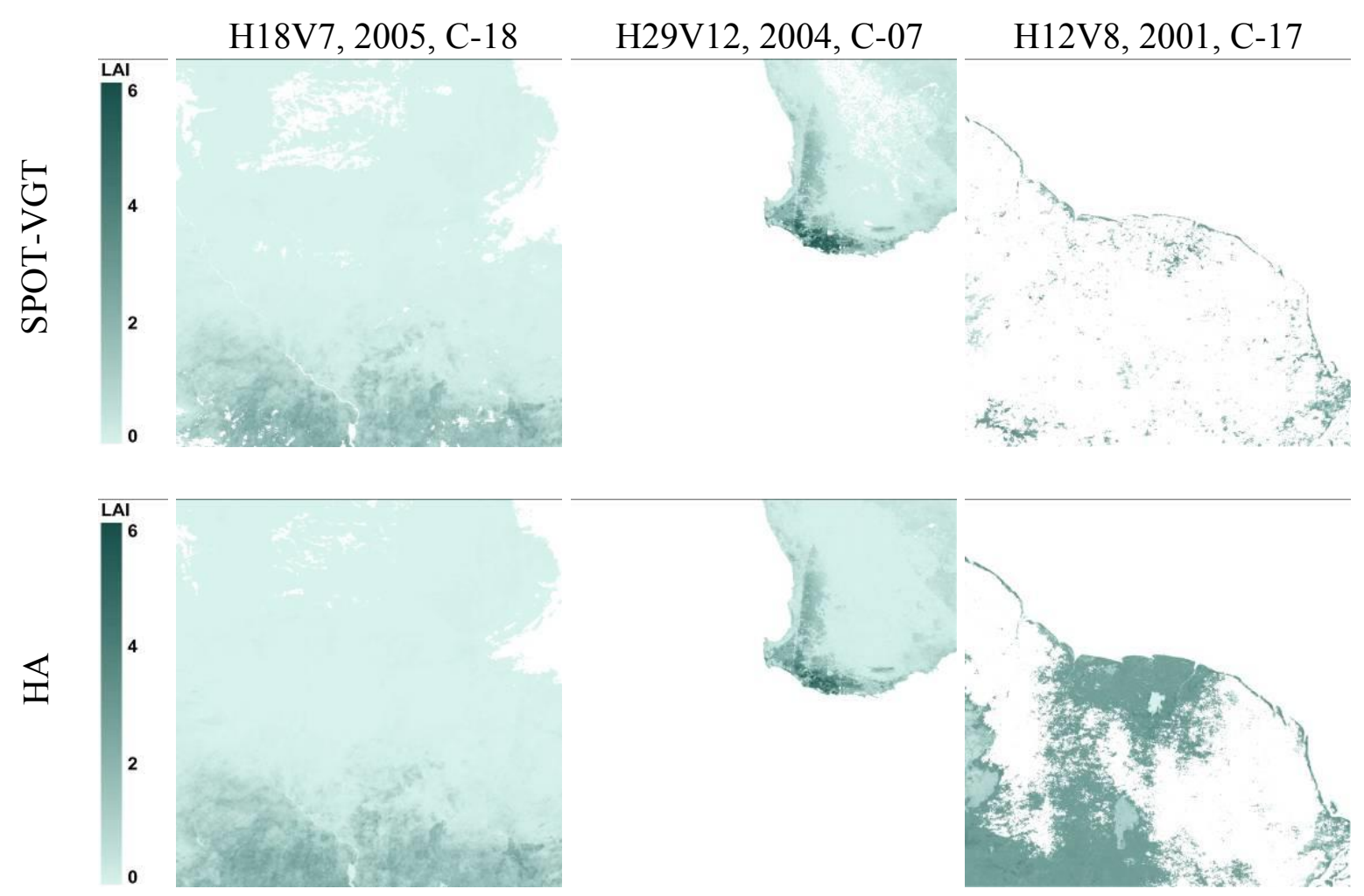

Abbildung 2.17. Vergleich von repräsentativen SPOT-VGT-Kacheln vor (erste Reihe, SPOT-VGT) und nach (zweite Reihe, HA) der Anwendung der Zeitreihenanalyse. Die Kacheln sind: Links, H18V7, Afrika; Mitte, H29V12, Australien; Rechts, H12V8, Südamerika. Die Kacheln und entsprechende Zeitpunkte sind oberhalb der Abbildung beschrieben. C-xx steht für das jeweilige Komposit des Jahres. Weiße Flächen stellen fehlende LAI-Werte bzw. Wasserpixel dar.

Dagegen zeigt der NRMSE (mittlere Reihe) für dieses Gebiet niedrige Werte, was belegt, dass die Ergebnisse der modifizierten HA über das gesamte Jahr gesehen dennoch gut mit den Originaldaten übereinstimmen und die Schwankungen nur einzelne Zeitschritte betreffen. Generell zeigt der NRMSE wie vorher beschrieben eher niedrige Werte unterhalb 0.50. Höhere Werte treten nur in gebirgigen Regionen, wie den Alpen (H19V4 in Abbildung 2.18), oder Regionen mit heterogenen meteorologischen Bedingungen, wie die trockenen Regionen in Nigeria (H18V7, Abbildung 2.19) auf. Bei Letzteren, sind die Niederschlagsereignisse im Süden stark an die Monsunzeit gekoppelt und werden im Norden durch die Passatwinde beeinflusst (Tetzlaff und Peters, 1986). Hinzu kommt eine sehr kurze Regenzeit von drei bis vier Monaten, unregelmäßige Verteilung der Niederschlagsereignisse und lange Trockenperioden über den Rest des Jahres. Bei Beginn der Regenzeit kommt es daher im zeitlichen Verlauf (beispielhaft für ein Pixel in der Sahelzone in Abbildung 2.20 dargestellt) des gemessenen LAI während der Trockenzeit bei niedrigen Werten von unter $0.1 \mathrm{zu}$ einem sprunghaften Anstieg von teilweise zwei Größenordnungen (auf über 2.5 für das 23. Komposit). 

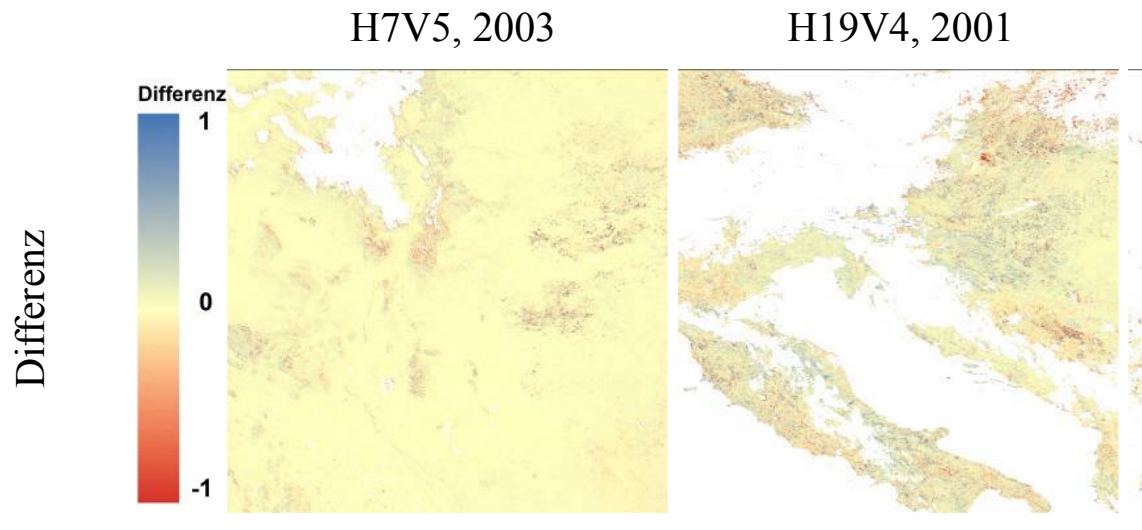

H12V8, 2001
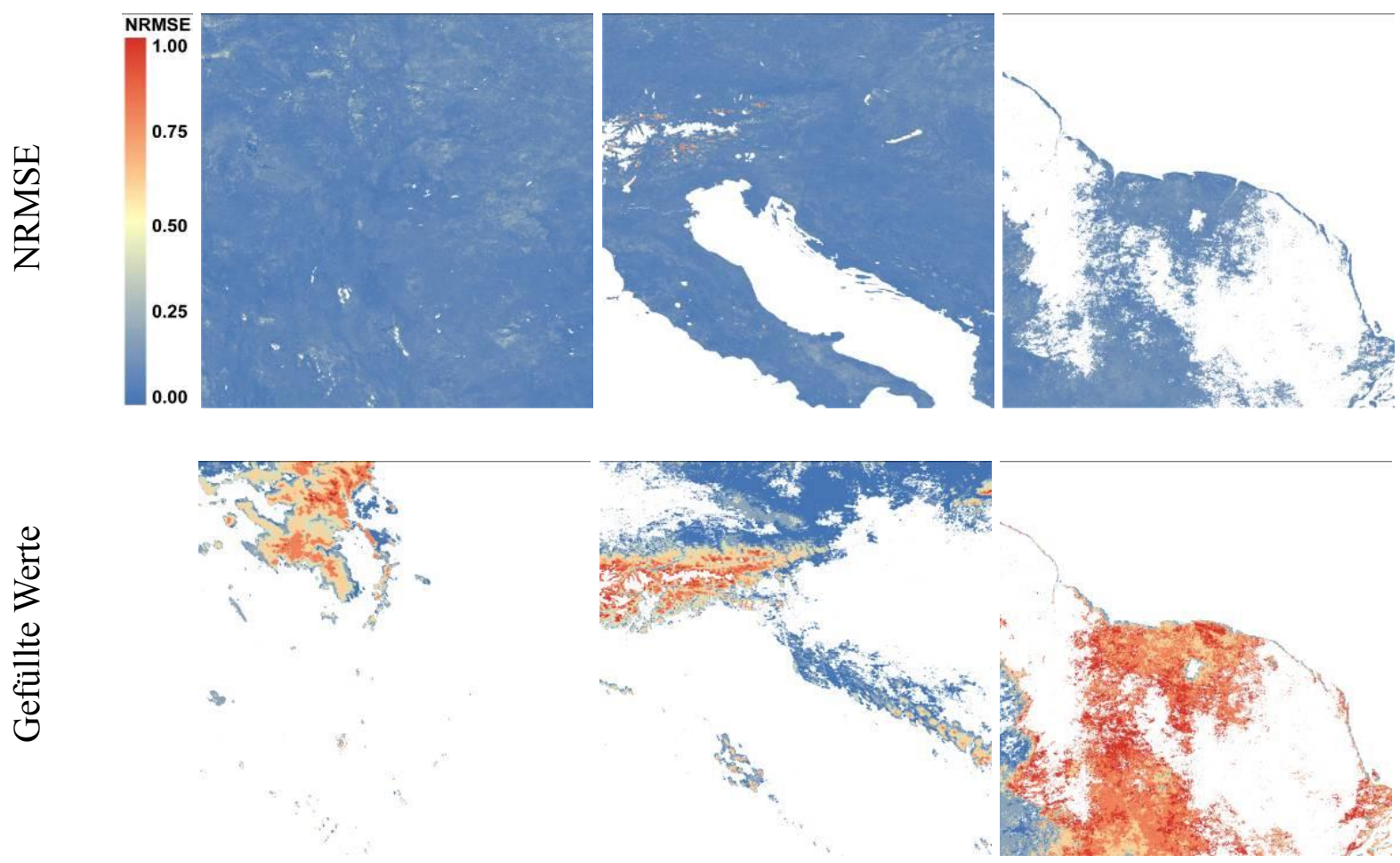

Anteil aufgefüllter Werte

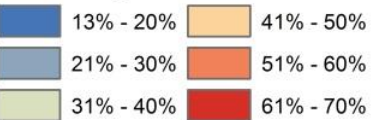

Abbildung 2.18. Differenz (SPOT-VGT - HA) (obere Reihe), NRMSE (mittlere Reihe) und Anteil an aufgefüllten Werten (untere Reihe) für die entsprechenden Jahre bestimmt für die in Abbildung 2.16 gezeigten Kacheln. Weiße Pixel zeigen Gebiete, für die keine HA berechnet wurde bzw. Gebiete für die keine Lücken aufgefüllt wurden. Die verschiedenen Kacheln und Jahre sind über den Abbildungen angegeben. 

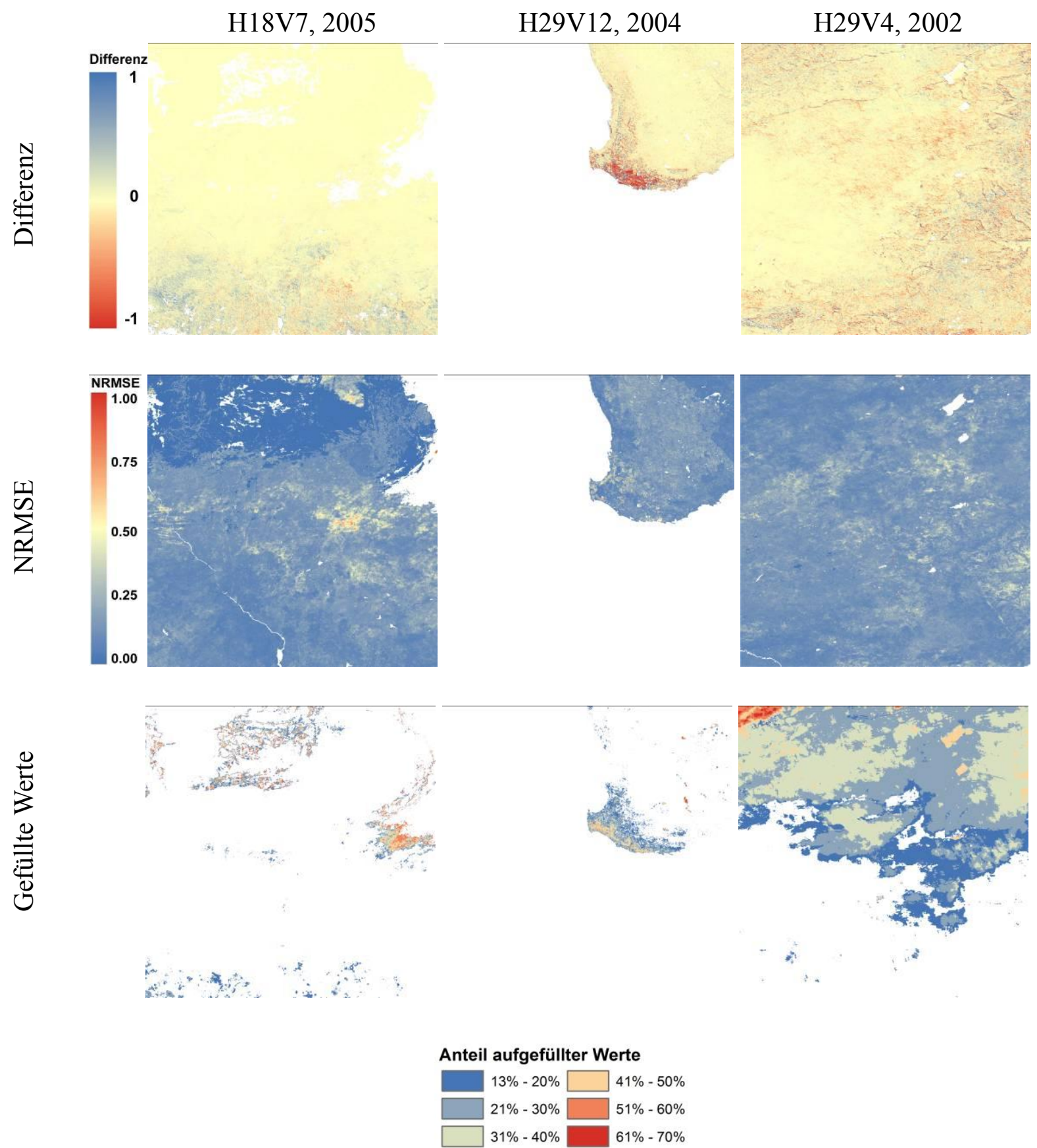

Abbildung 2.19. Differenz (SPOT-VGT - HA) (obere Reihe), NRMSE (mittlere Reihe) und Anteil an aufgefüllten Werten (untere Reihe) für die entsprechenden Jahre bestimmt für die in Abbildung 2.17 gezeigten Kacheln. Weiße Pixel zeigen Gebiete, für die keine HA berechnet wurde bzw. Gebiete für die keine Lücken aufgefüllt wurden. Die verschiedenen Kacheln und Jahre sind über den Abbildungen angegeben. 
Die sehr kurze Vegetationsperiode wird zwar bezüglich der Länge richtig dargestellt, allerdings ergibt sich durch die fälschliche Erkennung der Maximumswerte als Ausreißer eine zu niedrige Amplitude. Hinzu kommt, dass für die Darstellung der Vegetationsperiode im Vergleich zu den konstanten, sehr niedrigen Werten über die Trockenzeit, eine Schwingung mit verhältnismäßig großer Amplitude angenommen werden muss. Dies wirkt sich auch auf die übrige Zeitreihe aus, was dazu führt, dass die Schwingungen für die Phase mit niedrigen LAI-Werten auch ins Negative gehen würden. Dies wird dadurch abgefangen, dass solche Werte auf null gesetzt werden. Bei der Berechnung des RMSE und daraus des NRMSE ergibt sich dann für solche Zeitschritte ein Fehler von 100\%, was sich entsprechend auf den Gesamtfehler des Pixels und des Jahres auswirkt. Dies wird bei Betrachtung der globalen NRMSE-Karte (Abbildung 2.22b) für die gesamte Übergangszone zur Sahelwüste durch erhöhte NRMSE-Werte deutlich.

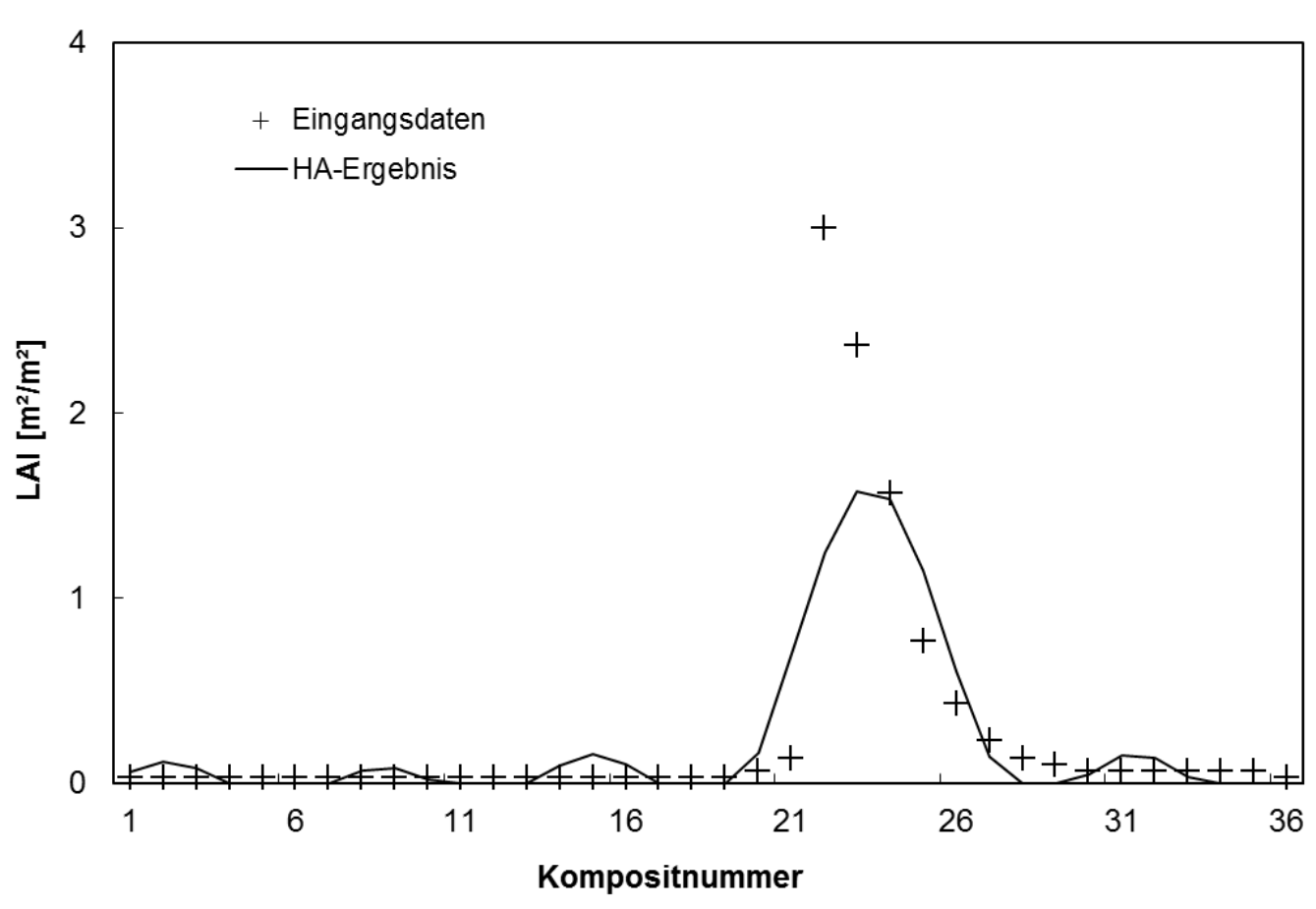

Abbildung 2.20. LAI-Zeitreihe für ein Pixel in der Übergangszone zur Sahel Wüste.

Die Anteile an aufgefüllten Lücken an der gesamten Zeitreihe sind in den Abbildung 2.18 und Abbildung 2.19 in der jeweils dritten Reihe dargestellt. Beispielsweise wurden in der Kachel H19V4 in 24\% der Pixel Werte aufgefüllt. Bei 57\% dieser Pixel waren die Lücken zwischen fünf und sieben Zeitschritten, bei $10 \%$ waren die Lücken größer als 18 Datenpunkte. Letztere treten hauptsächlich in der Alpenregion auf (orange und rote Bereiche). 
Bei näherer Betrachtung der NRMSE-Karte der Kachel H19V4 (Abbildung 2.18, obere Reihe, mittlere Spalte) fallen in der Umgebung von Seen vereinzelt hohe Werte auf, die darauf schließen lassen, dass die HA dort unverlässliche Ergebnisse erzeugt hat. Deutlich zu sehen ist dies anhand eines kleinen Ausschnittes dieser Kachel in Abbildung 2.21(c), die den NRMSE für die Region um den Neusiedler- und den Plattensee zeigt. Eine genauere Analyse hat gezeigt, dass es einen Versatz von etwa einem Pixel zwischen der LandWasser-Maske der GLC2000 (Abbildung 2.21a) und der LAI-Daten (Abbildung 2.21b) selbst gibt.
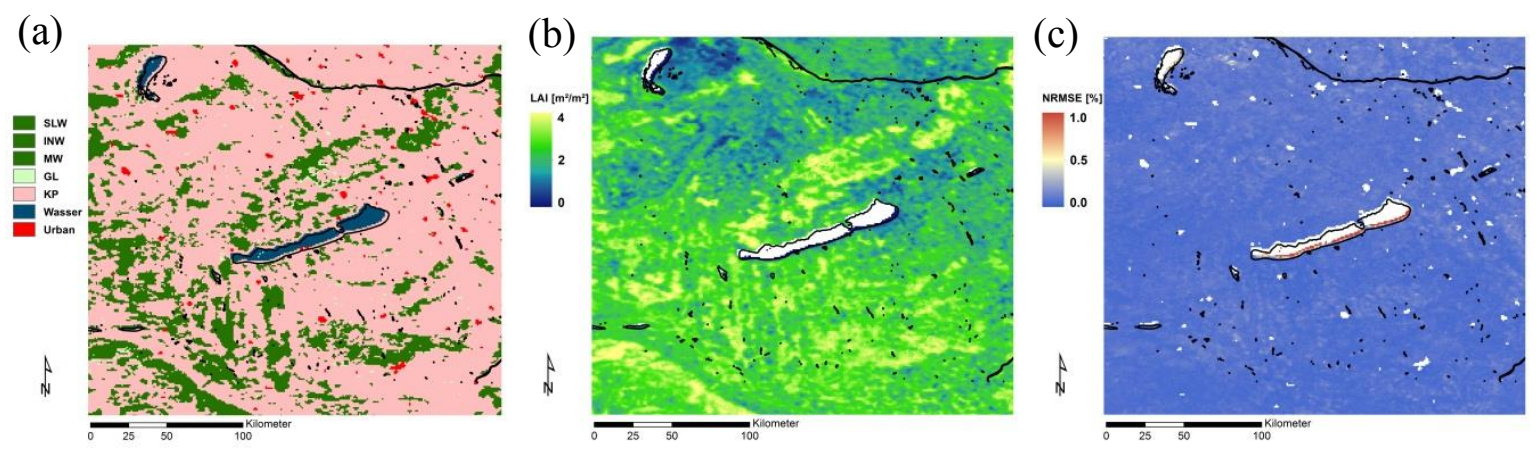

Abbildung 2.21: GLC2000 (a), LAI (18. Komposit für 2001) (b) und zugehöriger NRMSE (c) für den Plattensee (Ungarn, mittig) und den Neusiedlersee (Österreich, oben links im Bild). Schwarze Linien zeigen die SRTM-Wassermaske.

Dies zeigt sich deutlich anhand der überlagerten, hochgenauen Land-Wasser-Maske der Shuttle Radar Topography Mission (SRTM). Die Maske der GLC2000 ist demnach nach Nordwesten versetzt. Der beobachtete Versatz zwischen GLC2000 und LAI-Daten kann dazu führen, dass niedrige LAI-Werte an nordöstlichen Ufern von Seen, offensichtlich über Wasser gemessen werden (Abbildung 2.21b). Wie bereits vorher diskutiert (Abbildung 2.20) kann es bei sehr niedrigen LAI-Werten und hohen Variationen innerhalb einer Zeitreihe dazu kommen, dass die HA negative Werte für den LAI berechnet. Dies wird wiederum dadurch verhindert, dass diese Werte auf den Wert null gesetzt werden, was einen hohen NRMSE zur Folge hat. Der beschriebe Versatz der Land-Wasser-Masken ist für das CYCLOPES-Produkt für den Zeitraum 1999-2003 zu beobachten. Dieser Fehler wurde offensichtlich von 2004 an behoben, die alten Daten allerdings nicht neu prozessiert.

Um die Möglichkeit zu erhalten, das Modell BETHY/DLR global einsetzen zu können, wurde die neue Formulierung der HA schließlich auch auf den kompletten globalen SPOTVGT-LAI-Datensatz von CYCLOPES (1999-2007) und geoland2 (2010) angewandt. Damit konnte ein globaler Datensatz von räumlich und zeitlich kontinuierlichen LAI-Daten mit einer räumlichen Auflösung von $1 \mathrm{~km}^{2}$ für einen Zeitraum von elf Jahren erzeugt werden. Abbildung 2.22a zeigt eine globale Karte des LAI für den achtzehnten Zeitschritt, also 
Ende Juni, des Jahres 2001. Die vegetative Phase ist hier in der nördlichen Hemisphäre im Maximum und in der südlichen Hemisphäre im Minimum. Die von der HA berechneten LAI-Werte reichen von 0 bis $7.9 \mathrm{~m}^{2} \mathrm{~m}^{-2}$. Regionen, für die aufgrund von weniger als zehn verfügbaren Datenpunkten keine Zeitreihe berechnet werden konnten, sind hier in Äquatorialguinea, Kamerun und Gabun in Afrika zu erkennen sowie in Südamerika für das Amazonasbecken in Brasilien, Guyana, die Westküste von Kolumbien und Ecuador (vgl. Abbildung 2.17 rechte Spalte). In diesen Regionen sind die teilweise sehr großen Lücken die Folge von dauerhafter Wolkenbedeckung über nahezu das gesamte Jahr. In Abbildung 2.22b ist die globale Karte des NRMSE für $2001 \mathrm{zu}$ sehen.

Insgesamt zeigt sich, dass der HA-Prozessor für weite Teile der Erdoberfläche gute Ergebnisse erzielt, die in hoher Übereinstimmung mit den Originaldaten stehen. Die Verteilung des NRMSE auf Werte oberhalb 0.5 liegt für dieses Jahr bei etwa 1.1\% mit einem mittleren globalen Fehler von 0.12. Für den gesamten Zeitraum (1999-2007 und 2010) findet sich der höchste mittlere globale Fehler für das Jahr 2010 mit einem Wert von 0.14. Hier ist der Anteil an höheren Fehlern mit einem NRMSE von mehr als 0.5 bei $1.3 \%$.

Um die Qualität des Produktes unabhängig von den Originaldaten direkt zu bewerten, wurde es mit einem globalen Datensatz von Bodenmessungen des LAI verglichen (Tabelle 2.6). Diese Validierungspunkte wurden den Datenbanken der Projekte VALERI und BigFoot entnommen (erhältlich unter http://w3.avignon.inra.fr/valeri und http://www.daac.ornl.gov). Die Referenzkarten der Datenbanken wurden erzeugt, indem lokale Bodenmessungen mithilfe von hochaufgelösten Satellitenmessungen und einer Transferfunktion auf eine größere Fläche extrapoliert wurden (Morisette et al., 2006).

Die Größe der VALERI-Gebiete erstreckt sich auf jeweils $9 \mathrm{~km}^{2}(3 \mathrm{~km} \times 3 \mathrm{~km})$, die der BigFoot-Gebiete auf jeweils $25 \mathrm{~km}^{2}(5 \mathrm{~km}$ x $5 \mathrm{~km})$, wobei jedes Gebiet einen Eddykovarianzturm umschließt. Für den Vergleich wurden, nach den Richtlinien von Morisette et al. (2006), die LAI-Referenzwerte auf das Datum des SPOT-VGT-LAI-Produkts projiziert und danach nur solche Pixel für den Vergleich verwendet, die zu mehr als 75\% in dem zugehörigen SPOT-VGT-Pixel liegen. Fehler, die durch die Projizierung der Referenzdaten auftreten können, sollen minimiert werden, indem die räumlichen Mittelwerte für die einzelnen Gebiete berechnet werden. Diese sind in Tabelle 2.6 als „MAP“, „CYC“ und „HA“ bezeichnet, als die Mittelwerte der Referenzdaten, der SPOT-VGT-Originaldaten und der prozessierten SPOT-VGT-Daten. Für diesen direkten Vergleich wurden zunächst 79 LAIWerte von 40 weltweit verteilten Stationen analysiert. 


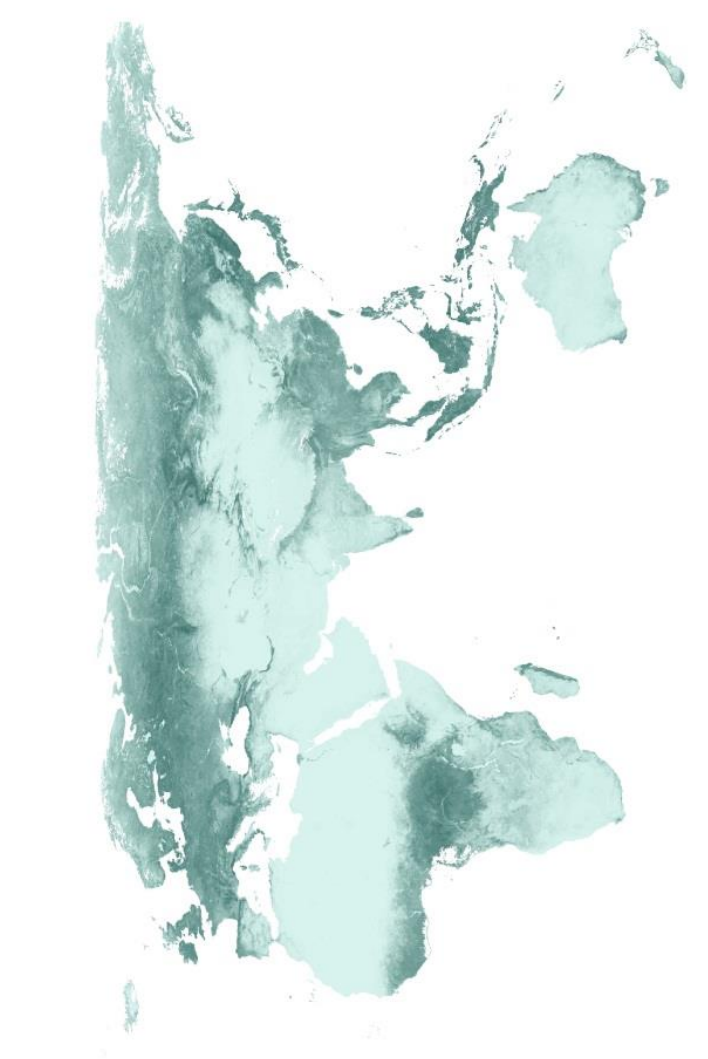

(a)
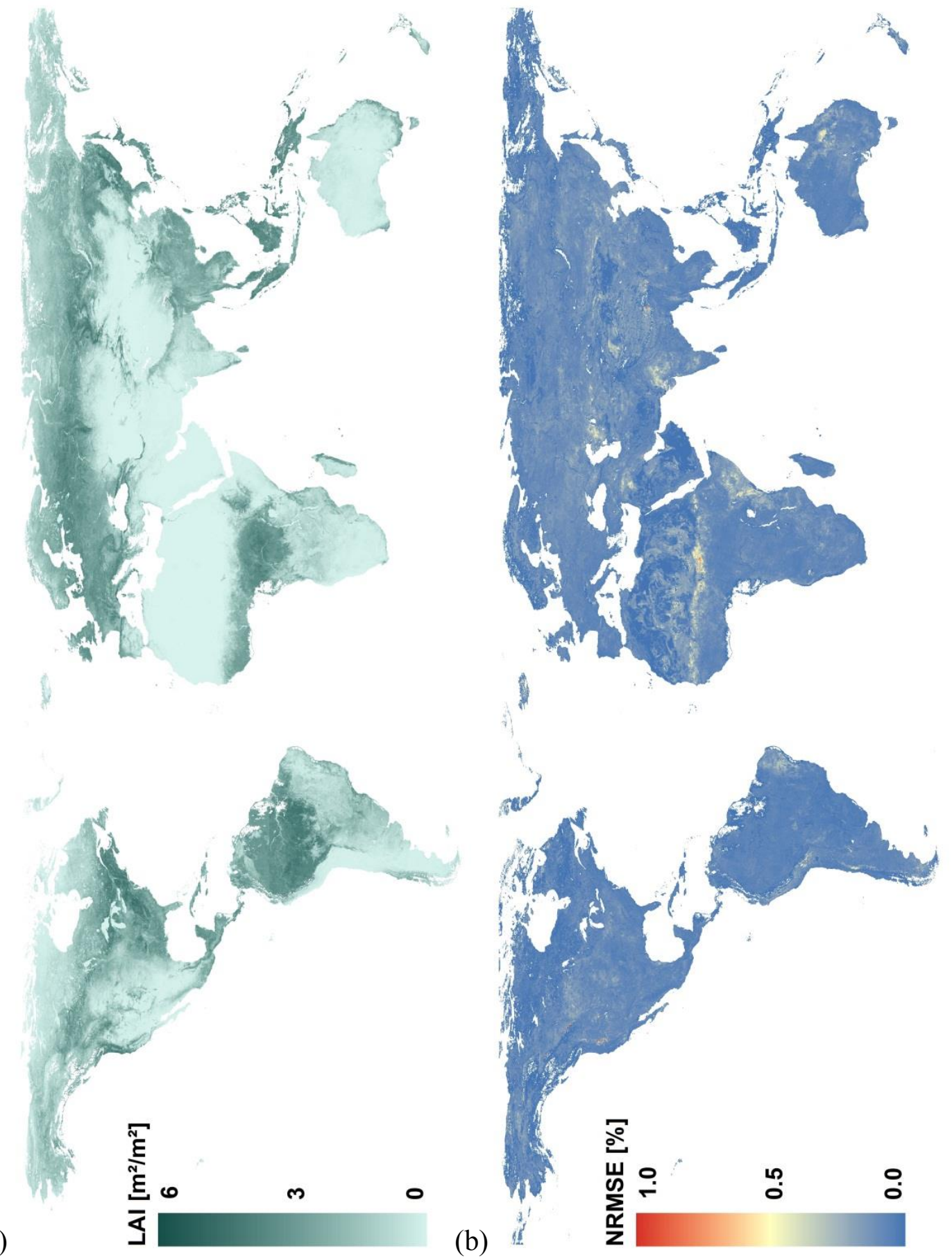

Abbildung 2.22: Globale Karten (a) des LAI des 18. Komposits und (b) des zugehörigen NRMSE für 2001. Weiße Pixel repräsentieren Werte für die keine Daten zur Verfügung stehen. 
Tabelle 2.6. Eigenschaften der Validierungsgebiete (insgesamt 24) und deren Referenzkarten (insgesamt 43). Die Abkürzungen der Überschrift sind: „Lat“ für Latitude, „Lon“ für Longitude, „LC“ für Landbedeckung (engl.: land cover). „MAP“ sind die Mittelwerte der LAI-Referenzkarten, „CYC“ die Mittelwerte der SPOT-VGT-Pixel und „HA“ die Mittelwerte der neu prozessierten SPOT-VGT-Pixel. Die Landbedeckungsklassen sind: „ILW" für immergrünen Laubwald „MW“ für Mischwald, „INW“ für immergrüner Nadelwald, „KP“ für Kulturpflanze und „GL“ für Grasland.

\begin{tabular}{|c|c|c|c|c|c|c|c|c|}
\hline Messgebiet & Land & Lat $\left[{ }^{\circ}\right]$ & Lon $\left[^{\circ}\right]$ & $\mathrm{LC}$ & Datum & Map & $\mathrm{CYC}$ & HA \\
\hline AekLoba & Sumatra & 2.63 & 99.58 & ILW & 2001.06 .01 & 3.3 & 3.1 & 3.4 \\
\hline Alpilles & Frankreich & 43.81 & 4.71 & KP & 2002.07 .20 & 1.4 & 1.2 & 1.2 \\
\hline Camerons & Australien & 32.6 & 116.25 & ILW & 2004.04 .06 & 2.2 & 1.8 & 1.7 \\
\hline Counami & Fr. Guyana & 5.34 & -53.24 & ILW & 2001.10 .18 & 4.8 & 2.7 & 3.1 \\
\hline Demmin & Deutschland & 53.89 & 13.21 & $\mathrm{KP}$ & 2004.07 .23 & 4.5 & 3.3 & 3.1 \\
\hline Donga & Benin & 9.77 & 1.75 & GL & 2005.06 .20 & 1.8 & 1.4 & 1.7 \\
\hline \multirow[t]{3}{*}{ Fundulea } & Rumänien & 44.41 & 26.59 & $\mathrm{KP}$ & 2001.03 .17 & 1.0 & 0.5 & 0.4 \\
\hline & & & & & 2001.05 .02 & 3.3 & 1.8 & 1.9 \\
\hline & & & & & 2002.06 .09 & 1.2 & 1.5 & 1.3 \\
\hline Gilching & Deutschland & 48.08 & 11.32 & KP & 2002.07 .08 & 5.5 & 2.7 & 2.8 \\
\hline Gnangara & Australien & -31.53 & 115.88 & ILW & 2004.03 .03 & 1.0 & 0.6 & 0.6 \\
\hline \multirow[t]{2}{*}{ Järvselja } & Estland & 58.3 & 27.26 & MW & 2000.08 .26 & 3.0 & 3.0 & 3.0 \\
\hline & & & & & 2002.07 .13 & 4.2 & 2.9 & 3.2 \\
\hline \multirow[t]{2}{*}{ Nezer } & Frankreich & 44.57 & -1.05 & INW & 2001.06 .20 & 3.4 & 2.9 & 2.9 \\
\hline & & & & & 2002.04 .21 & 2.4 & 1.2 & 1.5 \\
\hline PlandeDieu & Frankreich & 44.2 & 4.95 & KP & 2004.06 .29 & 1.3 & 0.5 & 0.6 \\
\hline Puechabon & Frankreich & 43.72 & 3.65 & MW & 2001.06 .12 & 3.0 & 1.9 & 1.9 \\
\hline Romilly & Frankreich & 48.45 & 3.8 & $\mathrm{KP}$ & 2000.06 .05 & 3.8 & 3.8 & 3.8 \\
\hline Sonian & Belgien & 50.77 & 4.41 & MW & 2004.07 .28 & 5.6 & 2.6 & 2.6 \\
\hline Sud-Oest & Frankreich & 43.51 & 1.24 & KP & 2002.07.20 & 1.7 & 2.1 & 1.8 \\
\hline \multirow[t]{3}{*}{ Turco } & Bolivien & -18.24 & -68.19 & GL & 2001.07 .31 & 0.3 & 0.1 & 0.1 \\
\hline & & & & & 2002.08 .29 & 0.1 & 0.0 & 0.1 \\
\hline & & & & & 2003.04 .25 & 0.1 & 0.1 & 0.2 \\
\hline Wankama & Niger & 13.65 & 2.64 & GL & 2005.06 .22 & 0.1 & 0.1 & 0.0 \\
\hline Zhang Bei & China & 41.28 & 114.69 & GL & 2002.08 .23 & 1.2 & 1.2 & 1.3 \\
\hline \multirow[t]{2}{*}{ AGRO } & USA & 40.01 & -88.29 & $\mathrm{KP}$ & 2000.07 .07 & 2.6 & 3.0 & 3.1 \\
\hline & & & & & 2000.08 .11 & 3.2 & 3.0 & 3.0 \\
\hline HARV & USA & 42.53 & -72.17 & MW & 2002.08 .24 & 4.3 & 3.8 & 4.0 \\
\hline \multirow[t]{4}{*}{ KONZ } & USA & 39.09 & -96.57 & GL & 2000.06 .06 & 2.2 & 2.1 & 2.1 \\
\hline & & & & & 2000.08 .26 & 2.0 & 1.3 & 1.3 \\
\hline & & & & & 2001.06 .18 & 2.8 & 2.4 & 2.3 \\
\hline & & & & & 2001.08 .16 & 2.6 & 1.8 & 1.8 \\
\hline \multirow[t]{3}{*}{ NOBS } & Kanada & 55.89 & -98.48 & INW & 2000.07 .14 & 3.5 & 2.4 & 2.2 \\
\hline & & & & & 2001.07 .14 & 3.5 & 2.4 & 2.4 \\
\hline & & & & & 2002.07 .14 & 3.2 & 2.3 & 2.2 \\
\hline \multirow[t]{8}{*}{ SEVI } & USA & 34.35 & -106.7 & GL & 2002.07 .26 & 0.1 & 0.1 & 0.1 \\
\hline & & & & & 2002.08 .22 & 0.3 & 0.1 & 0.2 \\
\hline & & & & & 2002.09 .09 & 0.4 & 0.1 & 0.2 \\
\hline & & & & & 2002.11 .15 & 0.3 & 0.1 & 0.1 \\
\hline & & & & & 2003.06 .23 & 0.1 & 0.1 & 0.1 \\
\hline & & & & & 2003.07 .28 & 0.1 & 0.1 & 0.1 \\
\hline & & & & & 2003.09 .15 & 0.1 & 0.1 & 0.1 \\
\hline & & & & & 2003.11 .21 & 0.1 & 0.0 & 0.1 \\
\hline
\end{tabular}


Abweichungen in der Landbedeckungsklassifizierung zwischen den Stationsbeschreibungen und der GLC2000 wurden als Fehlklassifizierung der GLC2000 gewertet. Die entsprechenden Stationen wurden daher in dem Vergleich nicht mit berücksichtigt. Dadurch reduzierten sich die Werte auf 43 von insgesamt 24 Stationen. Die Landbedeckungsklassifizierungen der Stationen sind: immergrüner Laubwald (ILW), immergrüner Nadelwald (INW), Mischwald (MW), Kulturpflanzen (KP) und Grasland (GL). Das Streudiagramm in Abbildung 2.23 zeigt den Vergleich der originalen und neu prozessierten SPOT-VGT-LAI-Daten mit den Werten der LAI-Referenzkarten. Es ist deutlich zu sehen, dass durch die Anwendung der HA auf die LAI-Daten keine signifikante Änderung der Qualität erfolgt. Das Bestimmtheitsmaß R² erhöht sich leicht von 0.82 auf 0.83, zudem verringert sich der RMSE von 0.92 auf 0.88. Die hohe Genauigkeit des SPOT-VGT-LAIProduktes konnte demnach erhalten werden.

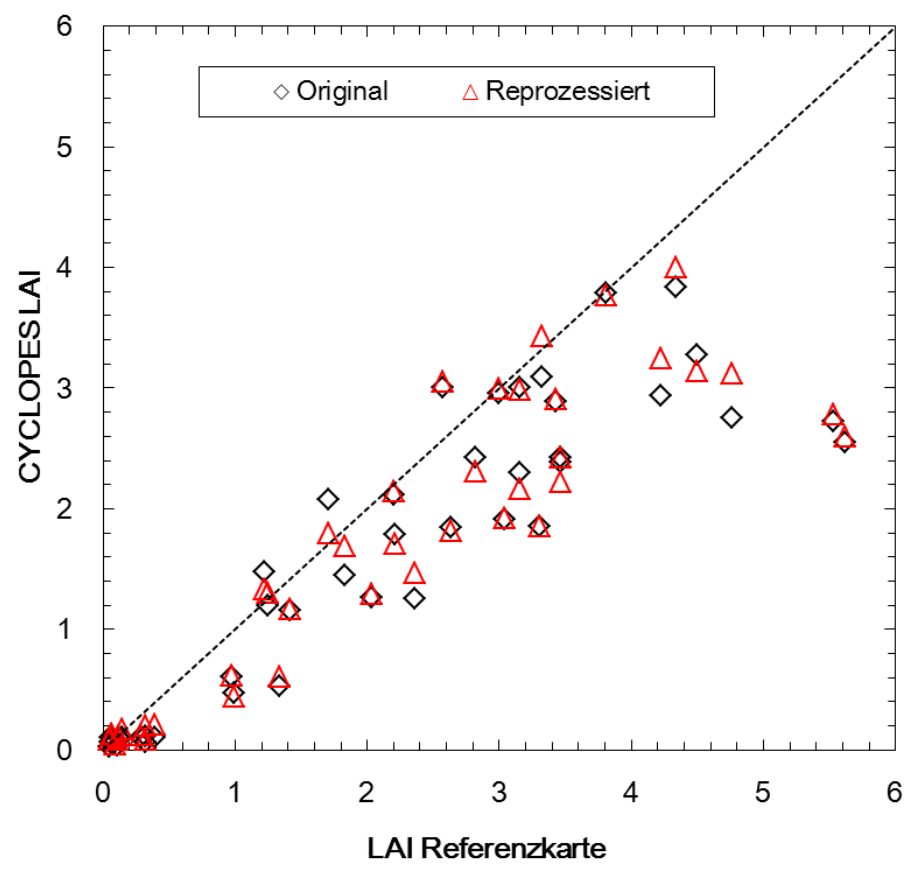

Abbildung 2.23. Streudiagramm des direkten Vergleichs von SPOT-VGT-Originaldaten (blaue Rauten) und neu prozessierten SPOT-VGT-Daten (rote Dreiecke) mit den Werten der LAIReferenzkarten.

Um die Güte des zeitlichen Verlaufes der neu prozessierten Daten zu diskutieren, sind in Abbildung 2.24 und Abbildung 2.25 die Zeitserien (1999-2007 und 2010) an acht der oben beschriebenen Stationen dargestellt. Die Beispiele repräsentieren jeweils zwei Vertreter der Haupt-Landbedeckungsklassen. Es wird deutlich, dass die SPOT-VGT-Daten durch die HA in erster Linie geglättet werden. Fluktuationen wie in den Beispielen Camerons und Nezer (Abbildung 2.25b) werden zwar nicht alle durch das Verfahren zur Erkennung von 
Ausreißern geglättet, sind nach Verger et al. (2011) dennoch unphysiologisch, vor allem da es sich hier um immergrüne Vegetation handelt. Diese werden durch die HA ebenfalls reduziert. Auch hier ist die gute Übereinstimmung der SPOT-VGT-LAI-Werte mit den Referenzwerten der Messstationen zu erkennen. Dennoch zeigt sich beispielsweise für die Fundulea Station (Abbildung 2.24a) nur bei der Messung für 2002 eine gute Übereinstimmung. In 2001 weichen sowohl die ursprünglichen SPOT-VGT- als auch die neu prozessierten Daten um einen Faktor von etwa zwei von den gemessenen Werten ab. Dies führt zu der Frage der Verlässlichkeit der Referenzkarten, die auch in Yuan et al. (2011) diskutiert wird. Garrigues et al. (2008) beziffert den Fehler in den LAI-Referenzkarten auf $\pm 20 \%$. Allerdings wurden Anteile von nicht-grüner Vegetation und der Effekt der Blatthäufung bei der Bestimmung des LAI in VALERI und BigFoot bereits berücksichtigt.

Einen Kritikpunkt der vorgestellten Vorprozessierung stellt die Verwendung der mittleren Phänologie der jeweiligen Landbedeckungsklasse innerhalb der entsprechenden Kachel zum Auffüllen von Datenlücken dar. Betrachtet man beispielsweise die Bewirtschaftung von Ackerland in der Kachel H19V4 werden hier im gemäßigten nördlichen Teil die Felder einmal im Jahr geerntet, im südlichen Italien hingegen oftmals zweimal im Jahr. Die Berechnung der mittleren Phänologie aus Werten der gesamten Kachel führt dazu, dass weder die eine noch die andere Art der Bewirtschaftung wiedergegeben wird. Dadurch können höhere Fehler auftreten, wenn in solchen Gebieten Lücken mit der mittleren Phänologie aufgefüllt werden müssen. Eine mögliche Verbesserung wäre eine Regionalisierung der Berechnung der mittleren Phänologie, wobei statt der Berechnung für ganze Kacheln die jeweiligen klimatischen Charakteristika berücksichtigt werden. Die Kachel H19V4 beinhaltet beispielsweise mehrere Klimazonen nach der Köppen-Geiger-Klassifizierung (Kottek et al., 2006). Der nördliche und östliche Teil (Süddeutschland, Österreich, Ungarn) ist als warmes Kontinentalklima eingestuft, während Italien, Kroatien und Albanien als trockenes Subtropen- oder Mittelmeerklima klassifiziert sind.

Trotz der aufgezeigten Schwachstellen konnte mit Hilfe der HA ein für global weite Teile kontinuierlicher Datensatz in räumlicher und zeitlicher Hinsicht erzeugt werden, der mit seinen hohen Qualitätsmerkmalen als verlässlicher Eingangsdatensatz für BETHY/DLR verwendet werden kann. 

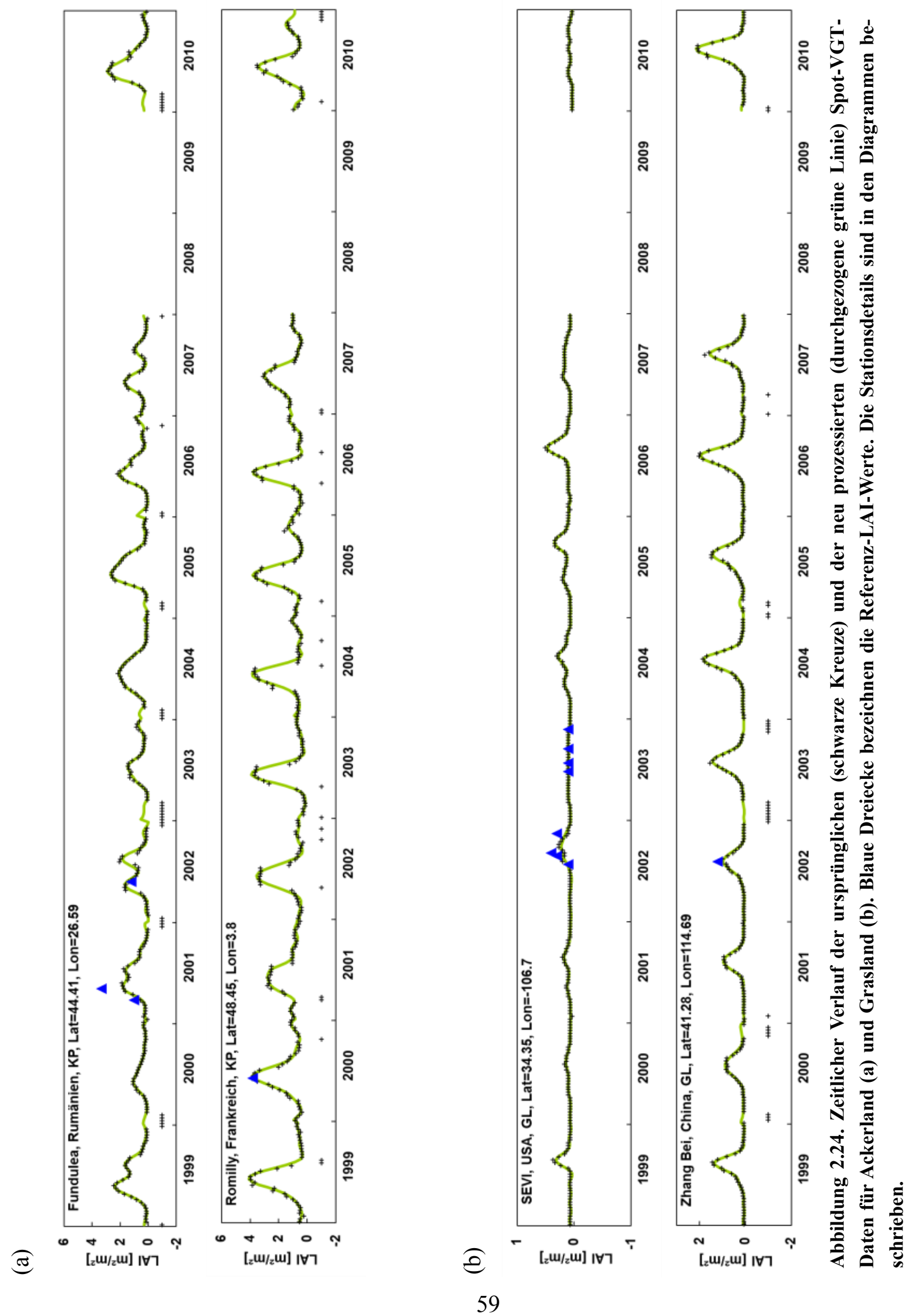

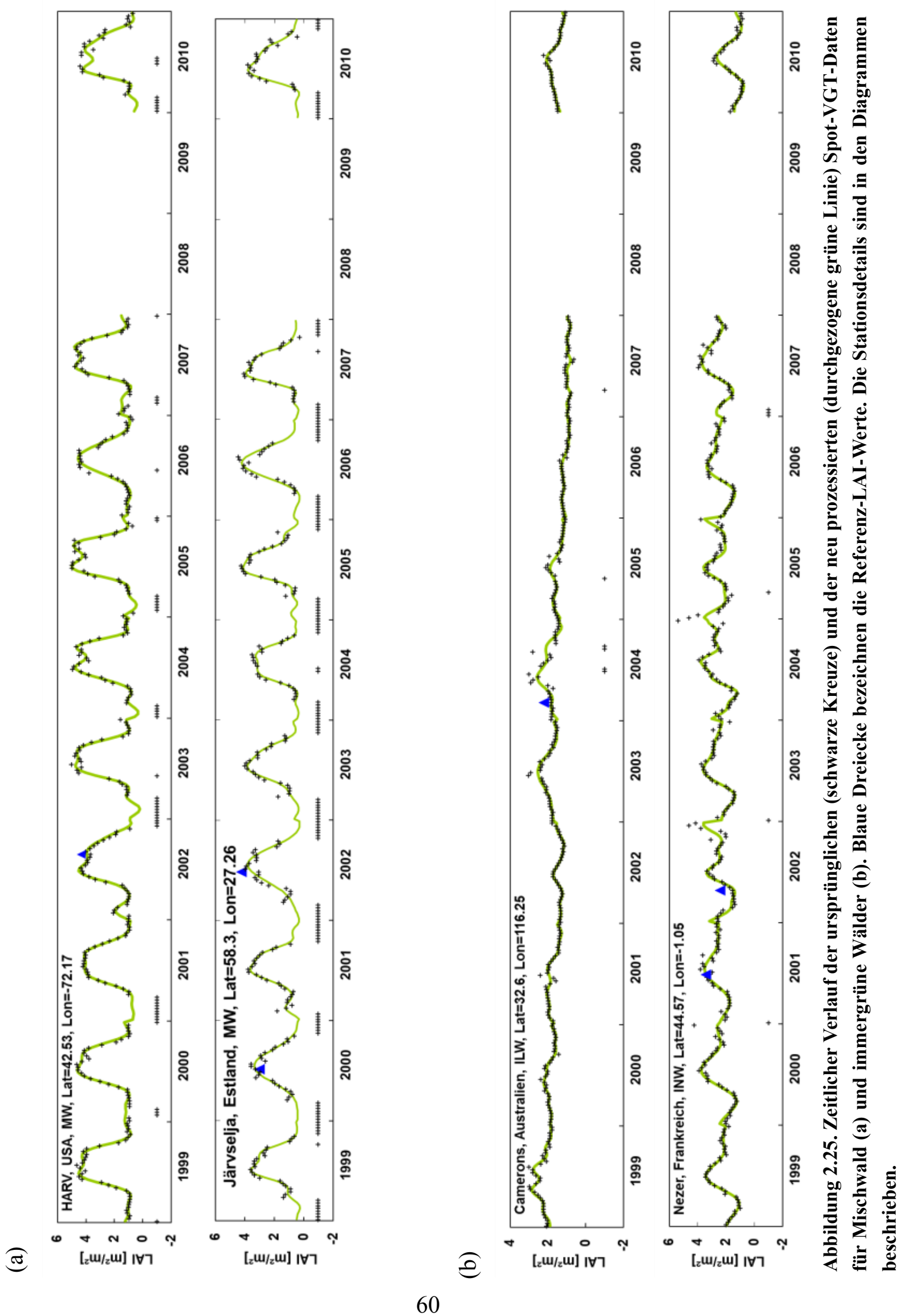


\subsubsection{Die Landnutzungsklassifikation}

Die Information über die Vegetationsverteilung wird aus dem Produkt des Joint Research Centre (JRC) der Global Land Cover für das Jahr 2000 (GLC2000) bezogen. Diese Landbedeckungsklassifikation wurde aus 19 regionalen Kartierungen zusammengestellt, die auf der SPOT-4-VEGETATION VEGA2000-Datenbank basiert (Mayaux et al., 2004; Bartholomé und Belward, 2005) und sich an das System der Landbedeckungsklassifikation (LCCS) von FAO (Food and Agriculture Organisation) und UNEP (United Nations Environment Programme) anlehnt (Di Gregorio und Jansen, 1998). Dabei wurde das Jahr 2000 als Referenzjahr verwendet. Die Genauigkeit des Produkts wird global mit 69\% angegeben (Mayaux, 2006) und ist folglich mit anderen global verfügbaren Produkten der Landbedeckungsklassifikation wie MODIS und Globcover (Tchuente, 2011) vergleichbar, wobei regional größere Abweichungen auftreten können.

Die GLC2000 unterscheidet insgesamt 23 Klassen, darunter 18 verschiedene Vegetationstypen und fünf Klassen ohne Vegetationsbedeckung (Tabelle 2.7).

Tabelle 2.7: Globale Landbedeckungsklassen der GLC2000 nach dem LCCS von FAO und UNEP.

\begin{tabular}{ll}
\hline Nummer & Landbedeckungsklasse \\
\hline 1. & Laubwald, immergrün \\
2. & Laubwald, sommergrün, geschlossen \\
3. & Laubwald, sommergrün, offen \\
4. & Nadelwald, immergrün \\
5. & Nadelwald, sommergrün \\
6. & Mischwald \\
7. & Wald, regelmäßig geflutet, Süßwasser \\
8. & Wald, regelmäßig geflutet, Salzwasser \\
9. & Mosaik: Wald / andere Vegetation \\
10. & Wald, abgebrannt \\
11. & Buschland, immergrün, geschlossen - offen \\
12. & Buschland, sommergrün, geschlossen - offen \\
13. & Krautige Vegetation, geschlossen - offen \\
14. & Krautige Vegetation oder spärliches Buschland \\
15. & Buschland und / oder krautige Vegetation, regelmäßig geflutet \\
16. & Kulturpflanzen \\
17. & Mosaik: Kulturpflanzen / Wald / andere Vegetation \\
18. & Mosaik: Kulturpflanzen / Busch- oder Grasland \\
19. & Freie Fläche \\
20. & Wasser (natürlich und künstlich) \\
21. & Schnee und Eis (natürlich und künstlich) \\
22. & Bebaute Fläche \\
23. & Salzsee \\
\hline
\end{tabular}


Innerhalb des Arbeitsgebietes treten davon nur 11 Vegetationstypen auf, die in der Tabelle gesondert markiert sind. Abbildung 2.26 zeigt diese Verteilung.

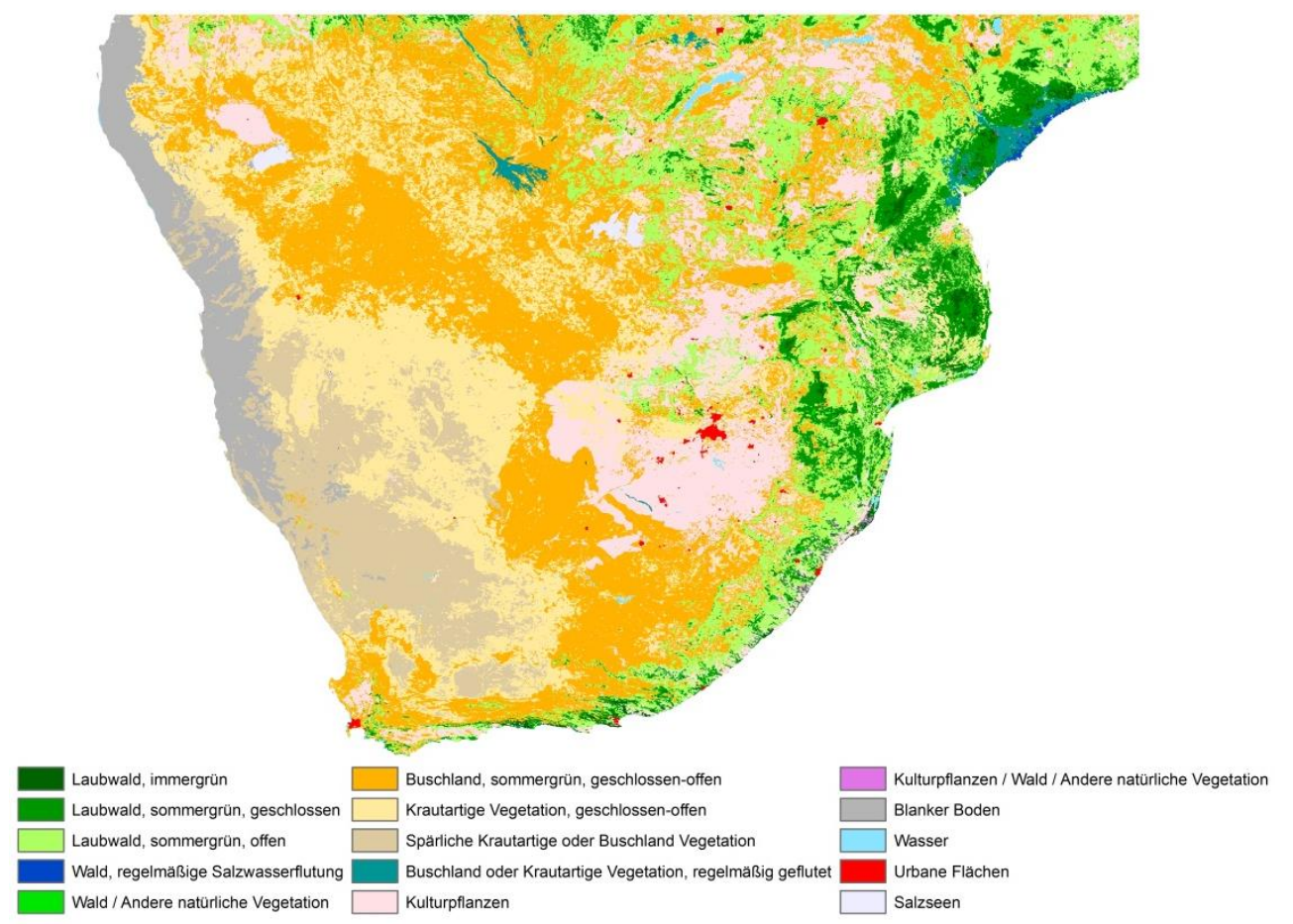

Abbildung 2.26: Verteilung der Vegetationsklassen nach GLC2000 innerhalb des Arbeitsgebietes.

Für die Zuweisung der bekannten pflanzen-physiologischen Parameter müssen die GLCTypen in die 33 vorhandenen BETHY/DLR-Klassen (Tabelle 2.4) übersetzt werden. Dabei kann je Pixel eine Primär- (PK) und eine Sekundärklasse (SK) definiert werden, um die heterogene Vegetationsverteilung innerhalb eines $1 \mathrm{~km}^{2}$ großen Pixels ansatzweise zu berücksichtigen. Die Übersetzung für das Arbeitsgebiet mit der Häufigkeit der einzelnen Klassen sowie der Aufteilung und der Gewichtung der Primär- und Sekundärklassen ist in Tabelle 2.8 aufgelistet.

Das Buschland ist nach der Klassifikation der GLC2000 der häufigste Vegetationstyp im Arbeitsgebiet, gefolgt von der krautartigen Vegetation, die als Grasland (Klasse 11) übersetzt wird. In Abbildung 2.26 zeigt sich, dass sich diese Vegetationstypen hauptsächlich über die westliche Hälfte des Arbeitsgebietes verteilen. Waldgebiete sind nur entlang der Südost-Küste, der Ost-Küste und ganz im Norden zu finden. Markant sind die regelmäßig gefluteten Regionen des Okawango-Deltas in Botswana und des Sambesi-Deltas in Mosambik. Die Verteilung der bewirtschafteten Flächen fokussiert sich auf die Region um die südafrikanischen Metropolen Johannesburg und Pretoria. Aber auch in Simbabwe und nördlich der Etosha-Ebene in Namibia ist der Anbau von Kulturpflanzen zu finden. 
Tabelle 2.8: Übersetzung der GLC2000-Vegetationstypen in Primär- (PK) und Sekundärklassen (SK) der BETHY/DLR-Vegetationstypen mit der jeweiligen Gewichtung $(g)$.

\begin{tabular}{lrrrrr}
\hline GLC2000 & Häufigkeit & PK & $g$ PK & SK & \multicolumn{1}{c}{$g$ SK } \\
\hline Laubwald, immergrün & 28463 & 3 & 0,7 & 11 & 0,2 \\
Laubwald, sommergrün, geschlossen & 273979 & 4 & 0,7 & 11 & 0,2 \\
Laubwald, sommergrün, offen & 641193 & 4 & 0,4 & 11 & 0,25 \\
Wald, regelmäßige Salzwasserflutung & 4854 & 4 & 0,7 & 34 & 0,1 \\
Wald / Andere natürliche Vegetation & 3931 & 4 & 0,4 & 8 & 0,4 \\
Buschland, sommergrün, geschlossen-offen & 1421014 & 8 & 0,5 & 11 & 0,25 \\
Krautartige Vegetation, geschlossen-offen & 899837 & 11 & 0,5 & 12 & 0,25 \\
Spärliche Krautartige oder Buschland-Vegetation & 393170 & 11 & 0,2 & 8 & 0,2 \\
Buschland oder krautartige Vegetation, & & & & & \\
regelmäßig geflutet & 34927 & 14 & 0,7 & 11 & 0,2 \\
Kulturpflanzen & 613816 & 15 & 0,7 & 34 & 0,1 \\
Kulturpflanzen / Wald / Andere nat. Vegetation & 2377 & 19 & 0,4 & 11 & 0,4 \\
\hline
\end{tabular}

Bei der Übersetzung von Grasland kann zwischen den Photosynthese-Mechanismen C3 und $\mathrm{C} 4$ sowie zwischen langen und kurzen Gräsern unterschieden werden. Im Arbeitsgebiet ist der C4-Mechanismus der dominante Typ. Darüber hinaus treten Kurzgräser $(\mathrm{h} \approx 30$ $\mathrm{cm})$ häufiger auf als Langgräser $(\mathrm{h} \approx 2 \mathrm{~m}$ ) (Trollope et al., 1989). Dementsprechend wird auch die Gewichtung der Grasarten festgelegt. Grasland wird auch als vorwiegende Sekundärvegetation für Waldpixel und Buschland verwendet. Beide Klassen nehmen vor allem bei der Betrachtung der Degradation durch die Beanspruchung der Vegetation als Weideland einen wichtigen Stellenwert in der Vegetationsverteilung ein.

\subsubsection{Die Bodeneigenschaften}

Die Information auf welchem Boden die Pflanzen wachsen ist ebenso wichtig wie das Wissen über die Vegetationsverteilung im jeweiligen Untersuchungsgebiet. Von den Bodeneigenschaften ist hauptsächlich abhängig, wie tief die Pflanze wurzeln und demnach wie gut sie mit Wasser versorgt werden kann. Außerdem wird im Bodenmodell die Rate des Wasserdurchsatzes in Abhängigkeit der Zusammensetzung aus Sand, Schluff und Ton berechnet. Daraus bestimmt sich auch die Wasserspeicherfähigkeit des Bodens. Die Information zu Zusammensetzung, Schichtung und Mächtigkeit der einzelnen Böden sowie zu deren Verteilung wird aus der Harmonized World Soil Database (HWSD), aus einer Zusammenarbeit von FAO und IIASA (International Institute for Applied Systems Analysis), bezogen (FAO et al., 2009). Die Informationen der HWSD stammen aus der digitalen Bodenkarte der Welt der FAO-UNESCO mit Aktualisierungen aus der European Soil Database (ESDB), der Bodenkarte der Chinese Academy of Sciences für China und regionalen 
SOTER Datenbanken. Die Zuverlässigkeit der HWSD wird von FAO et al. (2009) für das südliche Afrika, sowie für Lateinamerika und Zentral und Osteuropa am höchsten bewertet. Geringer fällt diese für Nordamerika, Australien, West Afrika und Asien aus.

Bei einer Auflösung von 30" x 30" (1 km x $1 \mathrm{~km}$ ) werden im Arbeitsgebiet 64 verschiedene Bodentypen unterschieden. Nach dem Schema von Tum et al. (2012) wird mit Hilfe dieser Parameter der Bodenwasserhaushalt berechnet. Die Formulierung als mehrschichtiges Modell stellt die wesentliche Erweiterung der ursprünglichen Version von BETHY/DLR dar. Durch die in Kapitel 2.3 vorgestellte Ergänzung zu diesem Modell, nach den Ansatz von Williams et al. (1984), ist es nun möglich auch Trockenstress während Dürreperioden zu berücksichtigen. Dieser darf für die Untersuchung von ariden Gebieten nicht außer Acht gelassen werden, wonach eine solche Anpassung in dieser Arbeit dringend erforderlich war. 


\subsection{Das Phänologiemodell}

Da beabsichtigt ist, das Modell BETHY/DLR zukünftig für die Szenarienberechnung zu verwenden, sind bestimmte Annahmen über die Eingangsdaten notwendig. Klimatische Größen wie Temperatur oder Niederschlagsverteilung sind meist direkt an die verschiedenen Klimaszenarien gekoppelt. Vegetationsindizes wie der notwendige Eingangsparameter des LAI sind allerdings nicht Bestandteil solcher Szenarien. Diese müssen demnach in eigenständigen Schemata innerhalb der Vegetationsmodelle berechnet werden. Als Vorbereitung der Anwendung von BETHY/DLR als Szenariomodell wird in dieser Arbeit ein solches Phänologiemodell ausgewählt, an die Modellanforderungen angepasst und als Modul in BETHY/DLR eingefügt.

Viele Phänologiemodelle verwenden für die Bestimmung des Beginns der Wachstumsphase und deren Verlauf die Wachstumsgradtage oder -zahlen (engl. Growing Degree Days, GDD) (Hunter und Lechowicz, 1992; Hannerz, 1999; Botta et al., 2000). Dabei wird die mittlere Tagestemperatur innerhalb eines festgelegten Zeitraumes aufsummiert, wenn diese über einem bestimmten Grenzwert liegt $\left(-5^{\circ} \mathrm{C} \leq T_{t h} \leq 10^{\circ} \mathrm{C}\right.$, je nach Biom). Der Zeitraum über den summiert wird, wird entsprechend der geographischen Lage auf den Übergang von der Ruhe- in die Wachstumsphase der Vegetation festgelegt. Wenn ein kritischer Wert $G D D_{c}$ erreicht ist, wird angenommen, dass das Blattwachstum einsetzt (Botta et al., 2000). Zur Kalibrierung der Modelle werden die Verteilungen der $G D D_{c^{-}}$-Werte dabei für unterschiedliche Biome aus satellitenbasierten Beobachtungen bestimmt. Die Notwendigkeit einen festen Zeitraum für die Summierung der Temperaturwerte festzulegen erschwert die Anwendung innerhalb des Modells BETHY/DLR, das möglichst flexibel auf globaler Ebene anwendbar bleiben soll.

Auch empirische Zusammenhänge zwischen der Länge der Ruhephasen und Temperatursummen wurden für die Modellierung zugrunde gelegt (Murray et al., 1989; Hunter und Lechowicz, 1992). Schließlich wurden auch Ansätze verfolgt, die die Abhängigkeit der Triebentwicklung der Pflanzen von äußeren Faktoren, wie der Temperatur und der Tageslänge berücksichtigen (Heide, 1993a,b; Hänninen, 1995). Für das zu implementierende Phänologiemodell gelten die Anforderungen, dass keine neuen Eingangsparameter notwendig sein sollen und die Berechnungen sich auf die bereits bestehende Datengrundlage stützen. Außerdem steht die flexible Anwendbarkeit in verschiedenen Gebieten der Erde im Vordergrund. Das Modell sollte also möglichst allgemeingültig für die unterschiedlichen Gegebenheiten anwendbar sein. Darüber hinaus soll das Modell auch die Limitierung des LAI durch Temperatur und Wasserverfügbarkeit berücksichtigen, ohne dabei zu komplex und rechenaufwendig zu werden. Das letztlich implementierte Modell baut auf dem Sche- 
ma nach Knorr et al. (2010) auf, das in der aktuellen Version des Carbon Cycle Data Assimilation System (CCDAS, Rayner et al., 2005) Modells verwendet wird. In dem Modell wird der phänologische Verlauf des LAI aus einer Kombination zweier Grenzwertparameter bestimmt. Dies sind die mittlere Tagestemperatur und die kumulierte, solare Einstrahlung die für die photosynthetische Aktivität der Pflanze relevant ist. In der Formulierung von Knorr et al. (2010) wird für die Abhängigkeit von der solaren Einstrahlung die Tageslänge verwendet. Als Anpassung des Modells wird für die Verwendung in BETHY/DLR die Tageslänge durch die PAR ersetzt. Durch diese Änderung können in der hier beschriebenen Version auch Variationen der Lichtverfügbarkeit aufgrund von Bewölkung berücksichtigt werden.

Wesentlicher Teil der Betrachtung ist der Übergang aus der Ruhe- in die Wachstumsphase. Im Gegensatz zu den GDD-Ansätzen ist hier zusätzlich zum Überschreiten einer Grenztemperatur $T_{\text {th }}$ auch die Überwindung einer minimalen Menge an photosynthetisch aktiver Strahlung $P A R_{t h}$ notwendig (Knorr et al., 2010). Die Entwicklung des LAI teilt sich also auf in zwei Phasen: der aktiven Phase, beschrieben durch die Funktion $f_{l}$ und der inaktiven Phase, beschrieben durch die Funktion $f_{2}$. Die Anteile der beiden Phasen lassen sich in integraler Form durch die Gaussschen Wahrscheinlichkeitsdichtefunktionen (engl. probability density function, PDF) $p$ und $q$ der Triggervariablen $T$ und $P A R$ ausdrücken. Diese werden pro Pixel für den gesamten Modellierungszeitraum berechnet. Nach Knorr et al. (2010) erhält man die Zeitabhängigkeit des LAI:

$$
\frac{d \Lambda(t)}{d t}=f_{1} \int_{-\infty}^{T} \int_{-\infty}^{P A R} p\left(T_{t h}\right) q\left(P A R_{t h}\right) d T_{t h} d P A R_{t h}+f_{2}\left(1-\int_{-\infty}^{T} \int_{-\infty}^{P A R} p\left(T_{t h}\right) q\left(P A R_{t h}\right) d T_{t h} d P A R_{t h}\right)
$$

$p$ ist bestimmt durch ihren Mittelwert $T_{t h}$ und ihre Standardabweichung $T_{r}$ der Temperatur. Die PDF $q$ wird durch den Mittelwert $P A R_{t h}$ und die Standardabweichung $P A R_{r}$ der photosynthetisch aktiven Strahlung bestimmt (Knorr et al., 2010). Die Integrale über die PDF entsprechen den Verteilungsfunktionen $\Phi$ der Standardnormalverteilung:

$$
\int_{-\infty}^{T} p\left(T_{t h}\right) d T_{t h}=\Phi\left(\frac{T-T_{t h}}{T_{r}}\right)
$$

und

$$
\int_{-\infty}^{P A R} q\left(P A R_{t h}\right) d P A R_{t h}=\Phi\left(\frac{P A R-P A R_{t h}}{P A R_{r}}\right)
$$


Das Produkt der beiden Funktionen (2.64) und (2.65) wird zu $f$ zusammengefasst wodurch sich Gleichung (2.63) vereinfacht zu:

$$
\frac{d \Lambda(t)}{d t}=f f_{1}+(1-f) f_{2}
$$

$f$ bestimmt dadurch den Anteil an Pflanzen innerhalb eines Pixels in der Wachstums- oder Erhaltungsphase (Knorr et al., 2010).

Die Verteilungsfunktionen $\Phi(x)$ werden über die Fehlerfunktion $\operatorname{erf}$ bestimmt:

$$
\Phi(x)=\frac{1}{\sqrt{2 \pi}} \int_{-\infty}^{x} e^{-t^{2} / 2} d t=\frac{1}{2}\left[\operatorname{erf}\left(\frac{x}{\sqrt{2}}\right)\right]
$$

Die Fehlerfunktion erf selbst kann nach der Herleitung von Winitzki (2003) genähert werden als:

$$
\operatorname{erf}(z) \approx \operatorname{sgn}(z) \sqrt{1-\exp \left(-z^{2} \frac{4 / \pi+a z^{2}}{1+a z^{2}}\right)}
$$

Durch die Signumfunktion wird dabei die Symmetrie der Fehlerfunktion $\operatorname{erf}(-z)=-\operatorname{erf}(z)$ respektiert. Die Konstante $a$ wird mit dem Wert 0.14 angegeben.

In Abbildung 2.27 und Abbildung 2.28 sind die PDF und die kumulativen Verteilungsfunktionen $\Phi(x)$ der Temperatur und der PAR als Beispiel für ein Pixel dargestellt.
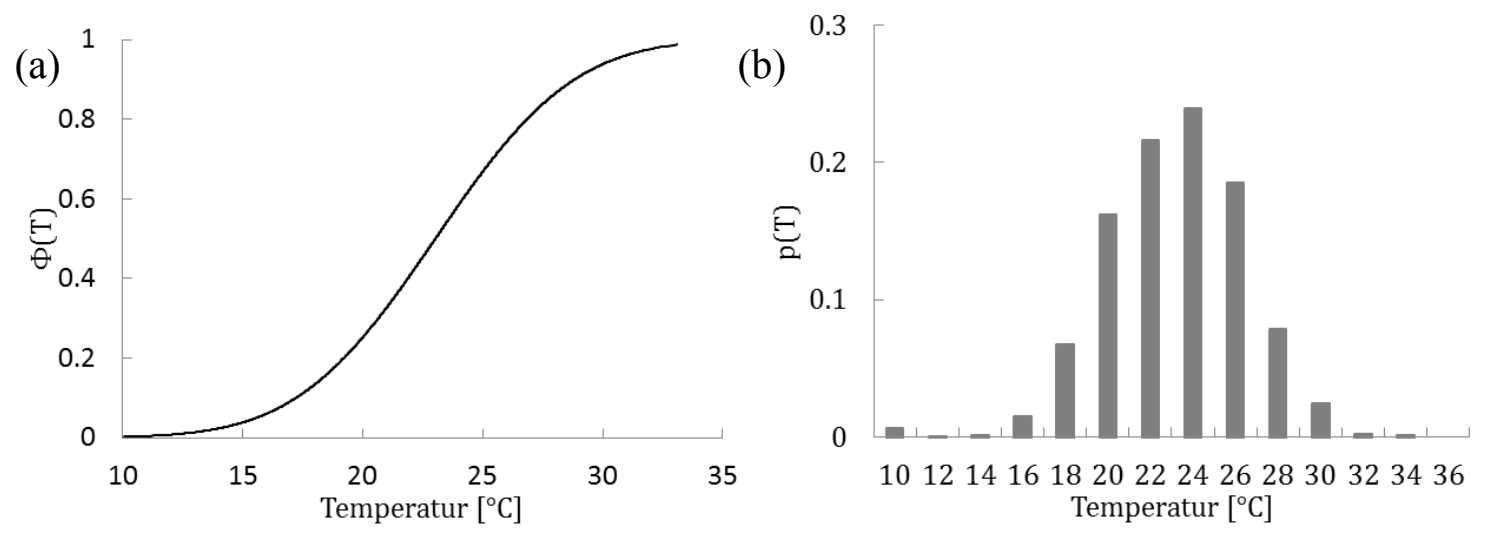

Abbildung 2.27: PDF (a) und Verteilungsfunktion (b) der Temperatur für ein Pixel im Arbeitsgebiet. 
(a)

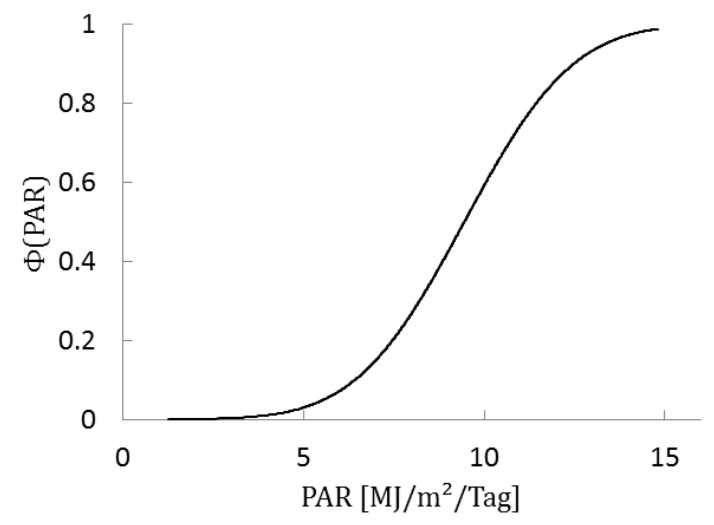

(b) 0.2

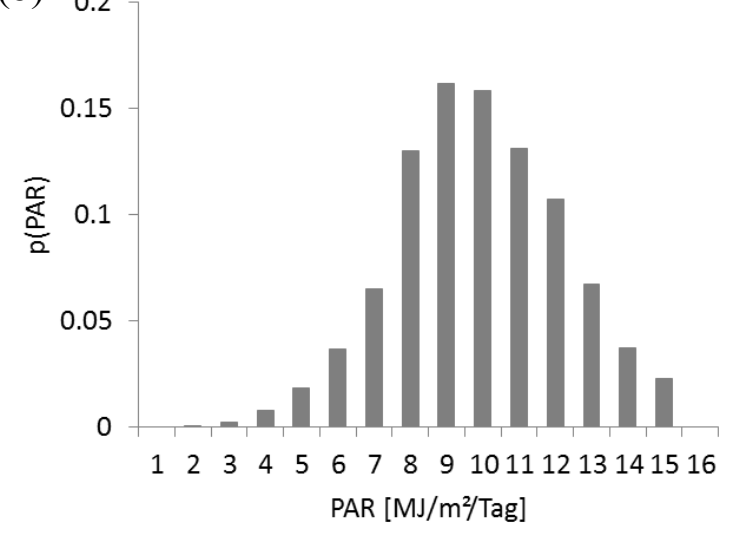

Abbildung 2.28: PDF (a) und Verteilungsfunktion (b) der PAR für ein Pixel im Arbeitsgebiet.

Zur Beschreibung der zeitlichen Entwicklung des LAIs einer Pflanze müssen schließlich die Funktionen $f_{1}$ (für Pflanzen in der Wachstumsphase) und $f_{2}$ (für Pflanzen in der Ruhephase) definiert werden. Die Wachstumsphase soll ohne Limitierung, beispielsweise durch die Substratverfügbarkeit, starten und einen maximalen LAI $\Lambda_{\max }$ erreichen, der sich durch Umweltfaktoren bestimmt (Knorr et al., 2010):

$$
f_{1}=\xi\left(\Lambda_{\max }-\Lambda\right)
$$

$\xi$ ist eine lineare Wachstumskonstante, die den Anstieg des LAI kurz nach dem Austreiben der Pflanze beschreibt. Die Ruhephase wird durch

$$
f_{2}=\frac{\Lambda}{\tau_{L}}
$$

beschrieben, wobei $\tau_{L}$ die Langlebigkeit der Blätter definiert. Hierdurch wird festgesetzt, wie schnell die Blätter abgeworfen werden oder diese inaktiv bleiben. Für sommergrüne Pflanzen liegt dieser Wert bei mehreren Tagen bis Wochen, bei immergrünen Pflanzen normalerweise im Bereich eines Jahres oder mehr (Woodward, 1987; Pallardy, 2008). Nach Einsetzen der beiden Funktionen $f_{l}$ und $f_{2}$ in Gleichung (2.66) erhält man

$$
\frac{d \Lambda(t)}{d t}=\xi\left(\Lambda_{\max }-\Lambda(t)\right) f-\frac{\Lambda(t)}{\tau_{L}}(1-f)
$$

was sich mit

$$
r=\xi f+\frac{(1-f)}{\tau_{L}}
$$

und 


$$
\Lambda_{\lim }=\frac{\xi \Lambda_{\max } f}{r}
$$

$\mathrm{Zu}$

$$
\frac{d \Lambda(t)}{d t}=r\left[\Lambda_{\lim }-\Lambda(t)\right]
$$

vereinfachen lässt (Knorr et al., 2010). Unter der Annahme, dass $f$ und $\Lambda_{\max }$ nicht von $t$ abhängig sind, ergibt sich hierfür die Lösung:

$$
\Lambda(t+\Delta t)=\Lambda_{\lim }-\left[\Lambda_{\lim }-\Lambda(t)\right] e^{-r \Delta t}
$$

Der Beginn der produktiven Phase der Vegetation wird, anstatt durch Wachstumsgradtage, über eine die Phänologie bestimmende Temperatur $T_{p h}$ und die einfallende photosynthetisch aktive Strahlung $P A R$ bestimmt (Knorr et al., 2010). $T_{p h}$ definiert sich als das Integral über die Lufttemperatur in zwei Metern Höhe $T_{2 m}$ mit exponentiell abfallendem Gewicht für zurückliegende Werte:

$$
T_{p h}(t)=\frac{1}{\tau_{m}} e^{-t / \tau_{m}} \int_{-\infty}^{t} T_{2 m}\left(t^{\prime}\right) e^{t^{\prime} / \tau_{m}} d t^{\prime}
$$

$\tau_{m}$ ist die Periode über die die Temperatur gemittelt wird. Ein geringerer Wert für $\tau_{m}$ führt dazu, dass der Temperaturtrigger aktuellere Temperaturdaten verwendet, der Grenzwert früher erreicht wird und damit die Vegetationsperiode nach vorn geschoben wird. Wenn allerdings $\tau_{m}$ zu kurz gewählt wird, beginnt $T_{p h}$ zu oszillieren, da kurzzeitige Variationen in $T_{2 m}$ nicht mehr gedämpft werden (Knorr et al., 2010). Zur einfacheren Berechnung kann obige Gleichung in eine inkrementelle Form gebracht und unter der Annahme, dass $T_{2 m}$ über den Zeitschritt des Modells $\Delta t$ konstant bleibt, vereinfacht werden zu:

$$
T_{p h}(t+\Delta t)=e^{-\Delta t / \tau_{m}} T(t)+T_{2 m}(t)\left(1-e^{-\Delta t / \tau_{m}}\right)
$$

Dadurch kann die Temperatur kontinuierlich unter Verwendung von aktuellen Werten von $T_{2 m}$ aktualisiert werden (Knorr et al., 2010). Im ersten Zeitschritt wird $T_{p h}=T_{2 m}$ gesetzt und eine Einschwing-Phase von einem Jahr gerechnet.

Als zusätzlicher Trigger für das Einsetzen des Pflanzenwachstums wurde bei Knorr et al. (2010) die Tageslänge genutzt. Alternativ wird in dem hier vorgestellten Modell in gleicher Weise die einfallende photosynthetisch aktive Strahlung verwendet. Zusätzlich zur Bedingung $T>T_{\text {th }}$ gilt hier $P A R>P A R_{t h}$. Hintergrund zur Verwendung der PAR als zweiten 
Parameter ist der Ansatz, dass die wärmste Periode über das Jahr generell später eintritt als der Zeitraum der maximalen Tageslänge bzw. der maximalen Tagessumme der PAR. Daher wird die Bildung der Blätter im Frühjahr über die Temperatur und das Verfärben im Herbst über die Photoperiode gesteuert. White et al. (1997) konnten zeigen, dass die Steuerung über die Tageslänge nur für Bäume und Sträucher in gemäßigten Gebieten aktiv ist. Für Gräser wird daher $P A R_{t h}=0$ gesetzt. Tropenpflanzen besitzen ebenfalls keine Adaption für niedrige Temperaturen, demnach keinen aktiven Schutz vor kalten Bedingungen. Daher wird für diese Pflanzen $T_{t h}=-\infty$ verwendet. Zunächst wurde nach Knorr et al. (2010) für Bäume in gemäßigten Regionen der Temperaturtrigger auf $10{ }^{\circ} \mathrm{C}$, für Sträucher auf $8{ }^{\circ} \mathrm{C}$ und für Gräser auf $2{ }^{\circ} \mathrm{C}$ festgelegt. Im Gegensatz zur ursprünglichen Version wird hier für jedes Pixel für jedes Jahr der Mittelwert und die Standardabweichung für Temperatur und PAR aus den vorhandenen ECMWF-Datensätzen berechnet, um die regionalen Anpassungen an die global unterschiedlichen Rahmenbedingungen zu berücksichtigen.

Ein weiterer Ansatz des Modells ist die Berücksichtigung von limitierenden Faktoren, wie das verfügbare Wasser, Fruchtbarkeit des Bodens oder strukturelle Eigenschaften (Knorr et al., 2010). Der wichtigste Faktor ist insbesondere im Arbeitsgebiet des südlichen Afrika die Wasserlimitierung und wird gesondert betrachtet. Alle übrigen Faktoren werden in einen konstanten pflanzenspezifischen Faktor $\hat{\Lambda}$ zusammengefasst, der als maximal erreichbarer Wert angenommen wird. Die Wasserlimitierung beinhaltet den Verlust von Wasser während der Photosynthese durch die Evapotranspiration über die Stomata. Außerdem bewirkt eine limitierte Wasserverfügbarkeit ein ansteigend negatives Bodenwasserpotential und dadurch ein höheres Potential die Blätter abzuwerfen. Für diese Parametrisierung wird ein Model verwendet, das auf Woodward (1987) zurückgeht. Verwendet werden tägliche Werte der potentiellen Evapotranspiration und des pflanzenverfügbaren Bodenwassers zur Ableitung des wasserlimitierten LAI. Die Berechnung erfolgt analog zu der von $T_{p h}$. Dadurch wird verhindert, dass der LAI zu stark auf variierende tägliche Bedingungen reagiert. Vielmehr sollen die mittelfristigen klimatischen Gegebenheiten widergegeben werden (Knorr et al., 2010).

Die Entwicklung der Blätter wird unterbrochen, wenn unzureichend Bodenwasser für die Verdunstung zur Verfügung steht. Dann wird bestimmt, wie lange die Pflanze mit einem bestimmten LAI und einer bestimmten Menge an verfügbarem Wasser ohne zusätzlichen Regen überleben kann. Diese Zeitspanne wird mit $\tau_{W}$ bezeichnet. Damit kann der wasserlimitierte LAI $\Lambda_{W}$ durch $E\left(\Lambda_{W}\right) \tau_{W}=W$ definiert werden. $W$ bezeichnet das pflanzenverfügbare Bodenwasser (Gleichung (2.3)). Um den Wasserverlust nach der Zeit $\tau_{W}$ zu be- 
stimmen, wird ein linearer Zusammenhang zwischen Evaporation $E$ und LAI $\Lambda$ angenommen (Knorr et al., 2010):

$$
E(\Lambda) \approx \frac{\tilde{E}}{\tilde{\Lambda}} \Lambda
$$

$\tilde{E}$ ist der Mittelwert der potentiellen Evapotranspiration, zuletzt berechnet bei einem LAIWert von $\tilde{\Lambda}$. Daraus folgt für den wasserlimitierten LAI $\Lambda_{W}$ :

$$
\Lambda_{W}=\frac{W \tilde{\Lambda}}{\tilde{E} \tau_{W}}
$$

$\tau_{W}$ steht für die maximale Zeitspanne einer Trockenheit, die die Pflanze ohne Wassereintrag überbrücken kann, bevor die Blätter beginnen sich zu verfärben. Nimmt man an, dass Pflanzen in gemäßigten Klimaten keine Anpassung an Trockenheit besitzen, bedeutet dies, dass das verfügbare Wasser auch für immer verfügbar bleiben wird, also $\tau_{W} \rightarrow 0$. Dies ist allerdings gleichbedeutend mit einem unendlich anwachsenden, wasserlimitierten LAI ( $\Lambda_{W} \rightarrow \infty$ in Gleichung (2.79)). Dies wird durch den limitierenden Strukturfaktor $\hat{\Lambda}$ abgefangen, der als der von der Pflanze maximal erreichbare LAI gilt:

$$
\tilde{\Lambda}_{\max }=\min \left(\hat{\Lambda}, \Lambda_{W}\right)
$$

Für tropische, immergrüne Pflanzentypen wird generell für $\tau_{W}$ eine Zeitspanne von einem Jahr angenommen, für Gräser und sommergrüne Tropenpflanzen zwischen einem und zwei Monaten (Knorr et al., 2010).

Gleichung (2.69) verwendet anstatt $\tilde{\Lambda}_{\max }$ dessen gewichtetes Integral über die Zeit $\Lambda_{\max }$, das in gleicher Weise wie $T_{p h}$ berechnet wird:

$$
\Lambda_{\max }(t)=\frac{1}{\tau_{s}} e^{-t / \tau_{s}} \int_{-\infty}^{t} \tilde{\Lambda}_{\max }\left(t^{\prime}\right) e^{t^{\prime} / \tau_{s}} d t^{\prime}
$$

Analog zu Gleichung (2.77) werden auch im Falle des maximalen LAI die täglichen Zeitschritte in inkrementeller Form bestimmt (Knorr et al., 2010):

$$
\Lambda_{\max }(t+\Delta t)=e^{-\Delta t / \tau_{s}} \Lambda_{\max }(t)+\tilde{\Lambda}_{\max }(t)\left(1-e^{-\Delta t / \tau_{s}}\right)
$$


Zuletzt werden $\mathrm{zu}$ geringe Werte abgefangen, die in der Modellformulierung von BETHY/DLR zu Instabilitäten führen können. Dazu wird ein minimaler LAI von $0.1 \mathrm{~m}^{2}$ $\mathrm{m}^{-2}$ angenommen.

Das beschriebene Schema zur Berechnung des LAI wurde modular in das Modell BETHY/DLR eingebaut und kann momentan parallel zu Standard-Modellläufen betrieben werden, ohne dass die errechneten LAI-Werte im Modell selbst Verwendung finden. Die Kalibrierung der Parametrisierung für die unterschiedlichen Vegetationstypen der Landbedeckungsklassifikation erfolgt zunächst über eine einfache Variation der Parameter des Phänologiemodells. Dies dient zum Test der Formulierung bevor im Anschluss an diese Arbeit die regionale Anpassung der Parameter über die Verwendung eines Datenassimilationsmoduls erfolgen soll. Dieses wird Informationen vorhandener Datensätze des LAI verwenden, um mit Hilfe der optimalen Parameter möglichst genau die entsprechenden Werte wiederzugeben. Die in der Kalibrierung berücksichtigten Parameter sind in Tabelle 2.9 mit dem Wertebereich und den Variationsschritten zusammengestellt.

Tabelle 2.9: Modellparameter die zur Kalibrierung variiert werden, mit dem Wertebereich [min; max] und den Variationsschritten $\Delta$.

\begin{tabular}{lrrrr}
\hline Parameter & Einheit & Min & Max & Schritte \\
\hline$\hat{\Lambda}$ & $\mathrm{m}^{2} \mathrm{~m}^{-2}$ & 2 & 10 & $2 ; 4 ; 6 ; 8 ; 10$ \\
$\tau_{s}$ & Tage & 0 & 100 & $0 ; 25 ; 50 ; 75 ; 100$ \\
$\tau_{m}$ & Tage & 0 & 100 & $0 ; 25 ; 50 ; 75 ; 100$ \\
$\tau_{L}$ & Tage & 0 & 300 & $0 ; 10 ; 100 ; 300$ \\
$\tau_{W}$ & Tage & 0 & 360 & $0 ; 60 ; 120 ; 180 ; 240 ; 300 ; 360$ \\
$\xi$ & - & 0.2 & 1 & $0.0 .4 ; 0.6 ; 0.8 ; 1.0$ \\
\hline
\end{tabular}

Diese einfache Variation ergibt 14000 mögliche Kombinationen der Parameterwerte, für die jeweils ein kompletter Modelldurchlauf notwendig ist. Dies schließt diese Art der Kalibrierung für die standardisierte Anwendung im Modell aus, soll aber im Rahmen der vorliegenden Arbeit für den Test der Formulierung genügen. Zur Kontrolle der errechneten LAI-Werte werden diese für die häufigsten Vegetationstypen der Landbedeckungsklassifikation (siehe Tabelle 2.8) jeweils für mehrere Pixel über den Zeitraum von 1999 bis 2007 mit den Werten des Eingangsdatensatzes von BETHY/DLR (SPOT-VGT-LAI) verglichen und der NRMSE berechnet. Als regionale Einschränkung werden Testpixel innerhalb des in Kapitel 2.2 vorgestellten Arbeitsgebietes dieser Arbeit verwendet.

Insgesamt werden etwa 400 Pixel, verteilt über das Arbeitsgebiet, in die Kalibration einbezogen, für die in Tabelle 2.10 aufgeführten Vegetationstypen. Für immergrünen Laubwald 
wurde die Lebensdauer der Blätter $\tau_{L}$ fest auf 300 Tage festgelegt, um starke Schwankungen zu vermeiden. Es ergeben sich für die sechs Parameter, die ebenfalls in Tabelle 2.10 aufgeführten Kombinationen, die den niedrigsten NRMSE aufwiesen.

Tabelle 2.10: Nach der Kalibrierung festgelegte Werte der Modellparameter für die HauptVegetationstypen des Arbeitsgebietes. Abkürzungen der Vegetationstypen sind: immergrüner Laubwald (ILW), sommergrüner Laubwald (SLW), sommergrünes Buschland (SB), Grasland (GL), Sumpfland (SL), Kulturpflanzen (KP).

\begin{tabular}{lrrrrrr}
\hline Vegetationstyp & $\hat{\Lambda}_{\left[\mathrm{m}^{2} \mathrm{~m}^{-2}\right]}$ & $\tau_{s}$ [Tage $]$ & $\tau_{m}$ [Tage $]$ & $\tau_{L}$ [Tage $]$ & $\tau_{W}$ [Tage $]$ & $\xi$ \\
\hline ILW, gemäßigt & 6 & 25 & 50 & 300 & 360 & 0.2 \\
SLW, gemäßigt & 4 & 25 & 50 & 100 & 0 & 0.6 \\
SB & 2 & 25 & 25 & 10 & 60 & 0.8 \\
GL, C4, lang & 4 & 25 & 0 & 10 & 60 & 0.6 \\
SL & 4 & 25 & 25 & 10 & 0 & 0.8 \\
KP & 4 & 25 & 0 & 100 & 0 & 0.6 \\
\hline
\end{tabular}

Wie in Abbildung 2.29 zu sehen ist, liegen die mittleren Fehler für die einzelnen Vegetationstypen unter 50\%. Die höchsten Fehler ergeben sich für immergrünen Laubwald und Kulturpflanzen mit $43.8 \%$ und $43.7 \%$.

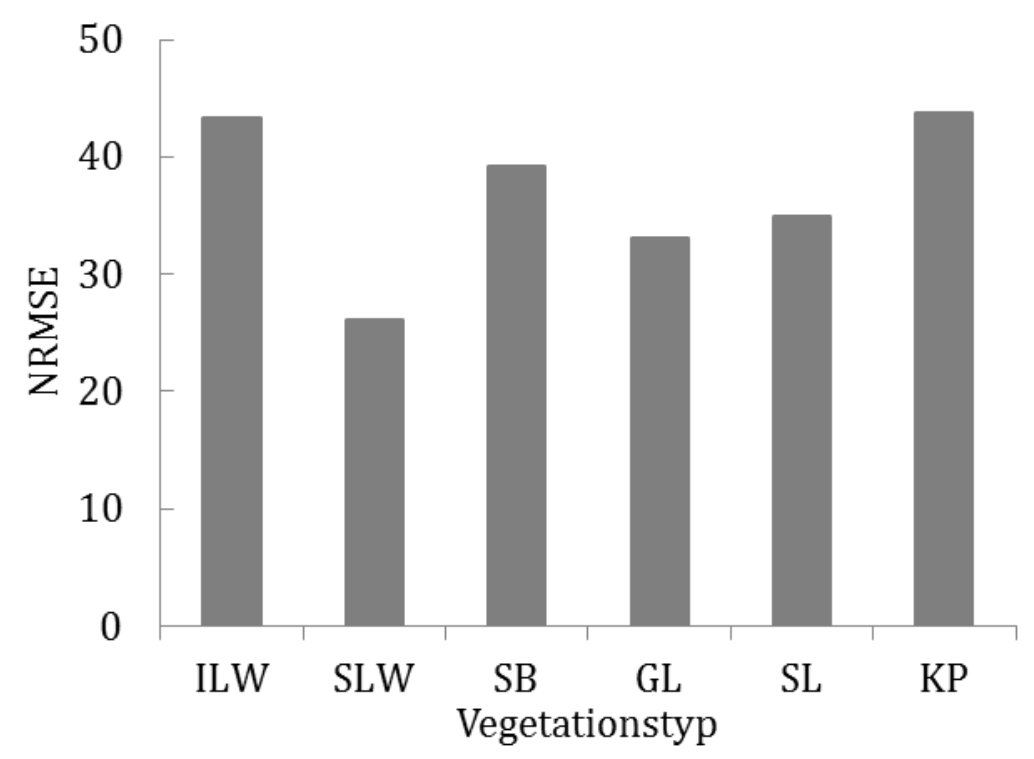

Abbildung 2.29: Mittlere Werte des NRMSE für die Haupt-Vegetationstypen des Arbeitsgebietes. Abkürzungen der Vegetationstypen sind: immergrüner Laubwald (ILW), sommergrüner Laubwald (SLW), sommergrünes Buschland (SB), Grasland (GL), Sumpfland (SL), Kulturpflanzen (KL). 
Im Falle des immergrünen Laubwaldes ergibt sich der hohe Fehler aus wahrscheinlichen Fehlklassifikationen der GLC2000, was auch in der zugehörigen Abbildung 2.30a zu erkennen ist. Dies zeigt ein generelles Problem der SPOT-VGT-LAI-Daten. Eine solche saisonale Variabilität des LAI lässt eher auf sommergrüne Vegetation schließen. Durch den festen Wert des Parameters $\tau_{L}$ in Gleichung (2.70) und der Variation der übrigen Parameter erhält man eine LAI-Zeitreihe mit entsprechender Amplitude der SPOT-LAI-Daten, aber deutlich geringerer Variabilität innerhalb des jährlichen Verlaufes.

(a)

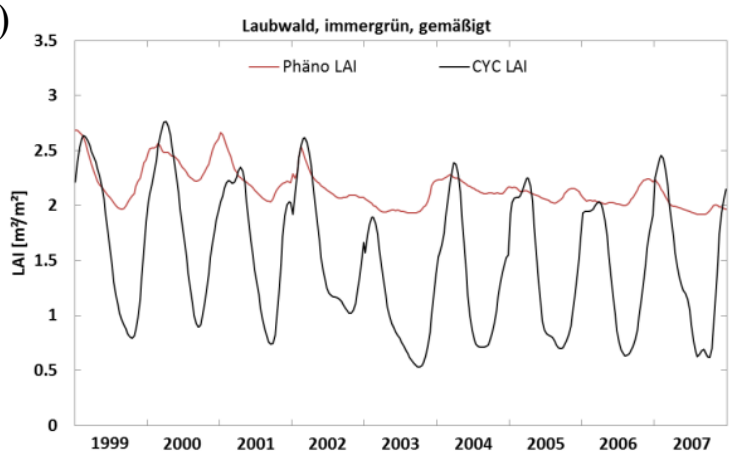

(c)

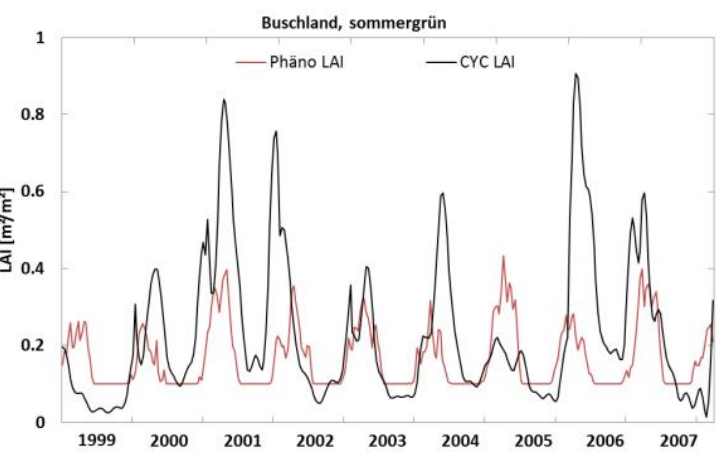

(e)

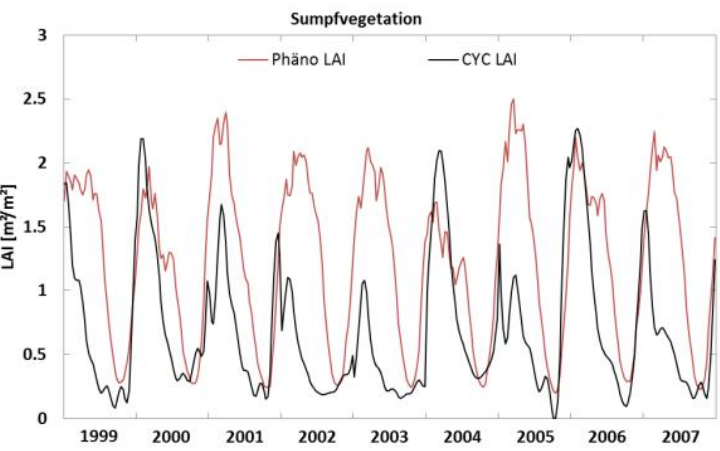

(b) ${ }_{4}$

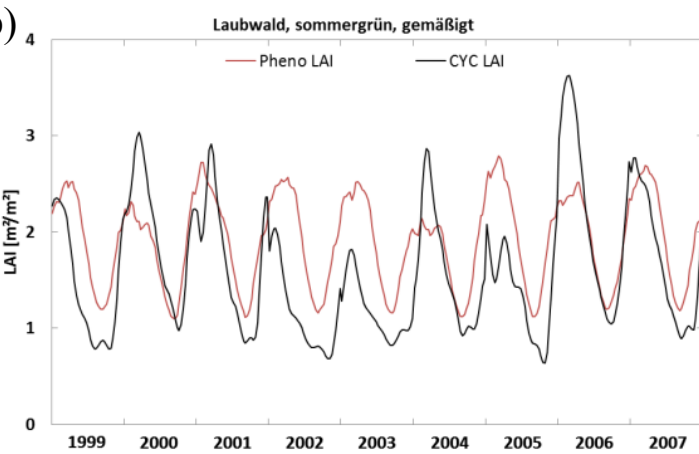

(d)

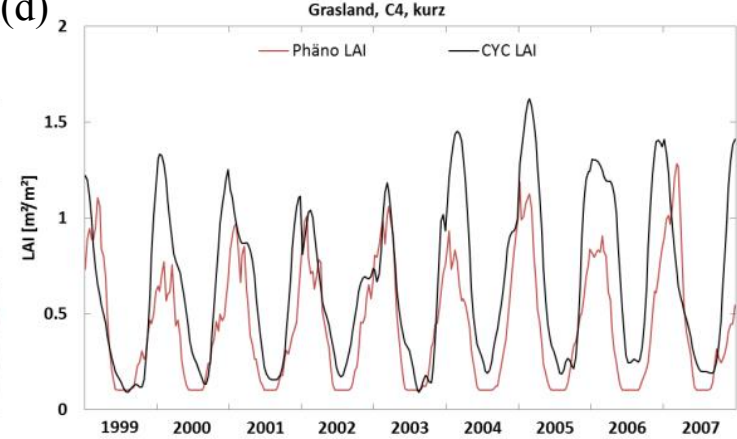

(f)

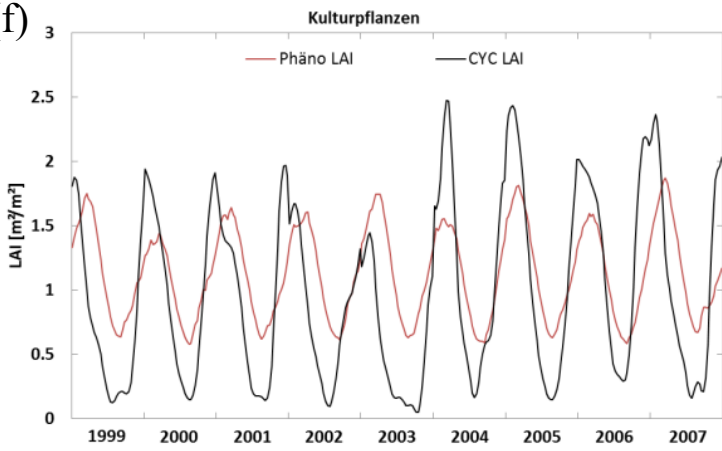

Abbildung 2.30: Vergleich der Zeitreihen aus den Originaldaten des SPOT-LAI und durch das Phänologiemodell berechnet. Gezeigt sind Beispiele der sechs häufigsten Vegetationstypen innerhalb des Arbeitsgebietes: (a) immergrüner Laubwald, gemäßigt (Klasse 3); (b) sommergrüner Laubwald, gemäBigt (Klasse 4); (c) sommergrünes Buschland (Klasse 8); (d) Grasland, C4, kurz (Klasse 11); (e) Sumpfland (Klasse 14); (f) Kulturpflanzen (Klasse 15). 
Der saisonale Verlauf von sommergrünem Buschland wird mit einem mittleren Fehler von 39.2\% wiedergegeben, Sumpfland und Grasland besitzen einen Fehler von 35.0\% bzw. $33.0 \%$ und den niedrigsten Fehler erzielt die Nachbildung der Phänologie von sommergrünem Laubwald, mit 26.1\%.Der immergrüne Laubwald erhält mit einem Wert von sechs den höchsten, maximalen LAI und mit 360 Tagen die längste Zeitspanne, die ohne Wassereintrag überstanden werden kann. Die niedrige Wachstumskonstante $\xi$ gibt die Eigenschaft wieder, dass die Blätter der immergrünen Vegetation nicht saisonal, sondern über die Jahre verteilt erneuert werden. Bis auf das Buschland mit $\hat{\Lambda}=2$ haben die übrigen Vegetationsklassen einen maximal erreichbaren LAI von vier. Dass für das Buschland ein so niedriger Wert des maximalen LAI angenommen wird, liegt vermutlich an der großflächigen Nutzung als Weideland. Dies führt zu starken Variationen im maximal erreichten LAI und dadurch auch zu sehr geringen saisonalen Werten für einzelne Jahre, was später anhand von Abbildung 2.30c diskutiert wird.

Die Phänologie des sommergrünen Laubwaldes wird, wie bereits erwähnt, ohne eine direkte Abhängigkeit vom Bodenwasser $\left(\tau_{W}=0\right)$ wiedergegeben. Für die Bestimmung der Temperatur $T_{p h}$, die den Beginn der Vegetationsphase steuert, ist nach den Ergebnissen der Kalibrierung ein Zeitraum von 50 Tagen optimal, die Lebensdauer der Blätter wird mit 100 Tagen am besten wiedergegeben. Die fehlende Abhängigkeit vom Bodenwasser ist auch für Sumpflandvegetation und Kulturpflanzen zu finden. Für diese Klassen ist davon auszugehen, dass tatsächlich permanent ausreichend Wasser zur Verfügung steht. Im Falle des Sumpflandes aufgrund der guten Speicherfähigkeit des Bodens, im Falle der Kulturpflanzen aufgrund der vermutlichen Bewässerung. Für das sommergrüne Buschland sowie für Grasland ergibt sich eine Zeitspanne von etwa zwei Monaten, die die Pflanzen ohne Wassereintrag überdauern können, im Mittel mit einer Lebensdauer der Blätter von 10 Tagen. Neben der Wasserlimitierung ist auch der Temperaturverlauf ausschlaggebend für die Phänologie des Buschlandes. Daher muss für die Bestimmung von $T_{p h}$ ein Zeitraum von 25 Tagen betrachtet werden. Für das Grasland fehlt diese Abhängigkeit von der Temperatur, wodurch schließlich die PAR neben der Wasserlimitierung die Rolle des bestimmenden Faktors übernimmt.

Beispiele für den aus diesen Werten berechneten Verlauf des LAI sind in Abbildung 2.30 für die sechs Vegetationstypen gezeigt. Zum Vergleich sind die entsprechenden Zeitreihen des LAI von SPOT-VGT dargestellt. Insgesamt zeigt sich, dass die saisonale Variabilität sehr gut simuliert werden kann. Schwierigkeiten ergeben sich bei den jährlichen Maxima der LAI Werte, die für die Vegetationstypen teilweise gut wiedergegeben werden, teilweise aber auch deutlich abweichen können. 
Probleme bereitet offensichtlich die Nachbildung der Zeitreihe von Buschlandvegetation (Abbildung 2.30c), die bereits mit einem mittleren Fehler von 39.2\% diskutiert wurde. Das gezeigte Beispiel hat einen NRMSE von $44 \%$, der sich hauptsächlich aus der Unterschätzung des maximalen, saisonalen LAI ergibt. Der Verlauf des SPOT-VGT-LAI zeigt eine starke Variation, die sich durch die Beweidung des Gebietes ergeben kann. Hier zeigt sich die Problematik, dass ohne zusätzliche Information über eventuelle Nutzung der jeweiligen Region als Weideland dies nicht durch das Phänologiemodell nachvollzogen werden kann. In diesem Falle müsste generell ein höherer, maximaler LAI angenommen werden und der Verlauf über Nutzungsdaten der einzelnen Jahre angepasst werden. Der phänologische Verlauf des Graslands kann dagegen gut mit einem NRMSE von $22 \%$ wiedergegeben werden. Für einzelne Jahre wird allerdings auch hier die Amplitude der Wachstumsphase unterschätzt. Dies gilt auch für den sommergrünen Laubwald, die Sumpfvegetation und die Kulturpflanzen. Die Übereinstimmung in der photosynthetisch aktiven Phase ist allgemein wichtiger als die Nachbildung des Minimums im Winter, da die Aktivität in dieser Jahreszeit ohnehin durch niedrige Temperaturen und PAR-Werte eingeschränkt wird.

Insgesamt wird deutlich, dass die saisonale Variabilität durch das Modell wiedergegeben werden kann, genaue Anpassungen an die Originaldaten allerdings problematisch sind. Dies ist allerdings auch nicht das Ziel, das mit dem Phänologiemodell verfolgt wird. Für die Szenariomodellierung ist es relevant den typischen Verlauf der verschiedenen Vegetationstypen mit der Länge der Vegetationsperiode und der Amplitude wiedergeben zu können. Dazu ist es allerdings notwendig das Modell in einem nächsten Schritt um ein Datenassimilationsverfahren zu erweitern. 


\section{$3 \quad$ Modellsensitivität}

Durch die Komplexität des Modells ist es nahezu unmöglich einzuordnen, welcher Eingangsparameter den größten Einfluss auf das Modellergebnis hat. Zwar wurde bereits in früheren Studien die Sensitivität des Modells auf Variationen der Eingangsparameter und Randbedingungen untersucht (Knorr, 1997; Wisskirchen, 2004), allerdings liegt inzwischen erneut eine modifizierte Version des Modells vor und mit dem Untersuchungsgebiet des südlichen Afrika eine Region, in der das Modell bisher noch nicht betrieben wurde. In der Arbeit von Knorr (1997) wurden verschiedene Modellvarianten auf veränderte Ergebnisse überprüft. Betrachtete Änderungen beinhalteten unter anderem eine Halbierung oder Verdoppelung der in dieser Modellversion konstanten Windgeschwindigkeit, die Vegetationshöhe und die Variation der Blattfläche. Die größte Auswirkung wurde bei Variation der autotrophen Respiration beobachtet. Eine höhere Atmungsrate $\left(f_{R, W}=0.3\right.$ in Gleichung (2.52) und $f_{R, \text { Blatt }}=0.3$ in Gleichung (2.50)) bewirkt eine geringere Produktivität, eine geringere Rate entsprechend eine höhere Produktivität. Die Abweichung vom Referenzwert entspricht dabei etwa bis zu 29\%. Die Verwendung eines alternativen Ansatzes des Photosynthesemodells nach Monteith (1977) bewirkt eine Erhöhung der Produktivität um etwa 21\%, die Halbierung bzw. Verdoppelung der Wurzeltiefe eine Verringerung bzw. Erhöhung um jeweils etwa 13\%. Nur geringe Auswirkungen zeigten in dieser Untersuchung Variationen der Schließgeschwindigkeit der Stomata (etwa -2\%), der Windgeschwindigkeit $( \pm 3 \%)$ oder der Luftfeuchtigkeit (etwa $+4 \%$ ). In dieser Studie wurden hauptsächlich modellintern konstante Größen bzw. feste Formulierungen auf globaler Skala getestet. Da diese bis auf die Windgeschwindigkeit unverändert blieben, werden sie im aktuellen Test nicht erneut berücksichtigt.

\subsection{Sensitivitätsstudie}

Als Testregionen wurden vier Stationen des FLUXNET-Messnetzwerkes verwendet, die im nachfolgenden Kapitel als Bewertungsgrundlage der Modellergebnisse dienen.

In Tabelle 3.1 und Tabelle 3.2 sind die Referenzwerte für diese Stationen zu finden, die in den folgenden Abbildungen bei 0\% Variation dargestellt werden. Im Rahmen der Sensitivitätsanalyse werden die Eingangsparameter jeweils um $\pm 5, \pm 10, \pm 20, \pm 25, \pm 50, \pm 75$ und \pm 100 Prozent des ursprünglichen Eingangswertes variiert und die Auswirkungen dieser Veränderungen auf die Modellergebnisse untersucht. Dabei werden die Eingangswerte zu den Zeitschritten geändert zu denen sie in das Modell eingehen. Die Analyse umfasst die Werte der Maximal-, Minimal- und mittleren Temperatur, den Niederschlag, die aktuelle PAR in Form von variierendem Bewölkungsgrad, die Windgeschwindigkeit, die Höhe über 
Normalnull und den LAI. Darüber hinaus wird auch die Landbedeckungsklassifikation variiert, woraus sich der Einfluss der Parameter $J_{\max }$ und $V_{c, \max }$ auf das Ergebnis ergibt. Bei der Betrachtung und Diskussion der jeweiligen Abbildungen ist die variierende Schrittweite zu beachten, wodurch der Verlauf der Kurven nicht kontinuierlich erscheint.

Tabelle 3.1: Eigenschaften der FLUXNET-Stationen mit geographischer Breite (Lat), geographischer Länge (Lon), der Landbedeckungsklassifikation (LCC) und der Höhe (h). Die Klassifikation wird unterschieden in sommergrünen Laubwald (SLW), Grasland (GL) und sommergrünes Buschland (SBL).

\begin{tabular}{llllll}
\hline Station & Land & Lat $\left[^{\circ}\right]$ & Lon $\left[^{\circ}\right]$ & LCC & $h$ [m ü. N.N.] \\
\hline Malopeni & Südafrika & $-23,83$ & 31,21 & SLW & 384 \\
Maun & Botsuana & $-19,92$ & 23,56 & GL & 946 \\
Mongu & Sambia & $-15,44$ & 23,25 & SLW & 1077 \\
Skukuza & Südafrika & $-25,02$ & 31,50 & SBL & 357 \\
\hline
\end{tabular}

Wesentliche Eigenschaften, wie geographische Lage, Landbedeckungsklassifikation und Höhe der Testpixel sind in Tabelle 3.1 zusammengefasst. Demnach befinden sich die beiden südafrikanischen Stationen Malopeni und Skukuza im Kruger Nationalpark, in dem Savannenbiome weit verbreitet sind. Die Stationen befinden sich auf vergleichbarem Höhenniveau. Ebenfalls als Savannenregion wurde das Gebiet der Station Maun in Botswana definiert. Mit 1077 m über N.N. ist Mongu die am höchsten gelegene Messstation. Das Untersuchungsgebiet in Sambia wird von sommergrünem Laubwald geprägt. Eine ausführlichere Betrachtung der einzelnen Stationen erfolgt für den Vergleich der Stationsmessungen mit den Modellergebnissen in Kapitel 4.2.

Tabelle 3.2: Langzeitmittel der einzelnen Parameter der Sensitivitätsanalyse für die Jahressummen des Niederschlags $(P)$ und für die Jahresmittelwerte der minimalen $\left(T_{\min }\right)$, der maximalen $\left(T_{\max }\right)$ und der mittleren Tagestemperatur $\left(T_{m i t}\right)$, des LAI, der Windgeschwindigkeit $(u)$ und dem Anteil an erreichter potentieller $\operatorname{PAR}\left(f_{P A R}\right)$.

\begin{tabular}{llllllll}
\hline Station & $P[\mathrm{~mm}]$ & $T_{\min }\left[{ }^{\circ} \mathrm{C}\right]$ & $T_{\max }\left[{ }^{\circ} \mathrm{C}\right]$ & $T_{\operatorname{mit}}\left[{ }^{\circ} \mathrm{C}\right]$ & $L A I\left[\mathrm{~m}^{2} \mathrm{~m}^{-2}\right]$ & $u\left[\mathrm{~m} \mathrm{~s}^{-1}\right]$ & $f_{P A R}$ \\
\hline Malopeni & 562 & 18,1 & 28,3 & 22,3 & 0,63 & 3,1 & $80 \%$ \\
Maun & 589 & 17,8 & 29,6 & 22,6 & 0,47 & 3,3 & $82 \%$ \\
Mongu & 1046 & 18,7 & 28,6 & 22,7 & 1,02 & 3,0 & $75 \%$ \\
Skukuza & 853 & 15,7 & 25,0 & 19,4 & 0,84 & 2,5 & $77 \%$ \\
\hline
\end{tabular}

Tabelle 3.2 enthält die phänologischen und klimatischen Langzeitmittelwerte der vier FLUXNET-Stationen über den Zeitraum der Jahre 1999 bis 2010. Berechnet wurden die Mittelwerte der Jahressummen des Niederschlags und Jahresmittel des LAI, der Windgeschwindigkeit, dem Anteil an erreichter potentieller PAR und der Temperatur. Diese dienen im Folgenden als Referenzwerte für die Variationen der Eingangsparameter. Des Weiteren sind die Modellergebnisse (GPP, NPP) bei unveränderten Eingangsparametern in Tabelle 
3.3 als Mittelwerte der Jahressummen der Jahre 1999 bis 2010 gelistet. Diese dienen als Vergleichswerte für die Ergebnisse der Modelläufe mit veränderten Variablen.

Tabelle 3.3: Modellergebnisse der GPP und der NPP $\left[\mathrm{gC} \mathrm{m}^{-2} \mathrm{Jahr}^{-1}\right]$ an den vier Stationen mit unveränderten Eingangsparametern als Referenzwerte.

\begin{tabular}{lllll}
\hline & Malopeni & Maun & Mongu & Skukuza \\
\hline GPP & 466.1 & 278.5 & 629.7 & 617.1 \\
NPP & 235.6 & 137.5 & 286.4 & 261.0 \\
\hline
\end{tabular}

\subsubsection{Die Höhe über Normalnull}

Die Höhe über Normalnull wird in BETHY/DLR dazu verwendet, den Luftdruck des aktuell beobachteten Pixels zu bestimmen. Dazu wird die barometrische Höhenformel (Gleichung (2.54)) verwendet, in die auch die mittlere Tagestemperatur eingeht.
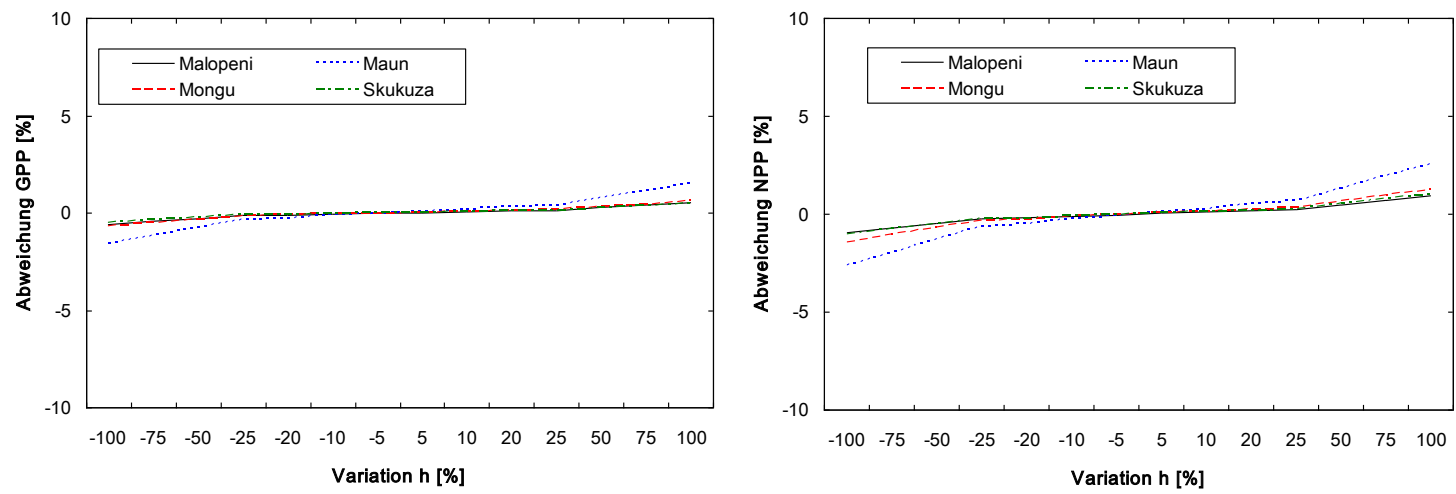

Abbildung 3.1: Mittlere Abweichung der Modellergebnisse GPP (links) und NPP (rechts) vom Referenzwert bei der Variation der Höhe über dem Meeresspiegel.

Wie in Abbildung $3.1 \mathrm{zu}$ erkennen ist, wirkt sich eine Variation der Höhe nur minimal auf die Modellergebnisse (GPP, links; NPP, rechts) aus. Die Unterschiede an maximaler Abweichung richten sich in diesem Fall hauptsächlich nach der unterschiedlichen Ausgangsgröße von GPP bzw. NPP (vgl. Tabelle 3.3). Maun hat für beide Größen den niedrigsten Wert der Stationen, erfährt aber mit der zweithöchsten Höhe der Stationen eine Änderung von etwa $1000 \mathrm{~m}$ im Maximum. Daraus ergibt sich die größte Variation für diese Station, die dennoch mit etwa 1.5\% für die GPP und etwa 2.5\% für die NPP äußerst gering ausfällt. 


\subsubsection{Der Blattflächenindex}

Der LAI ist einer der zentralen Parameter des Modells und bestimmt die Grundgrößen der Kohlenstoffassimilationsrate $A_{c}$ (Gleichung (2.39)) und der Bestandsleitfähigkeit $G_{c}$ (Gleichung (2.33)). Er wird ausßerdem bei der Berechnung des Skalierungsfaktors $f_{N}$, der Verteilung von RuBisCO innerhalb des Blattbestandes, benötigt, sowie für die Berechnung der thermischen Transmissivität der Vegetation für die einfallende solare Strahlung.
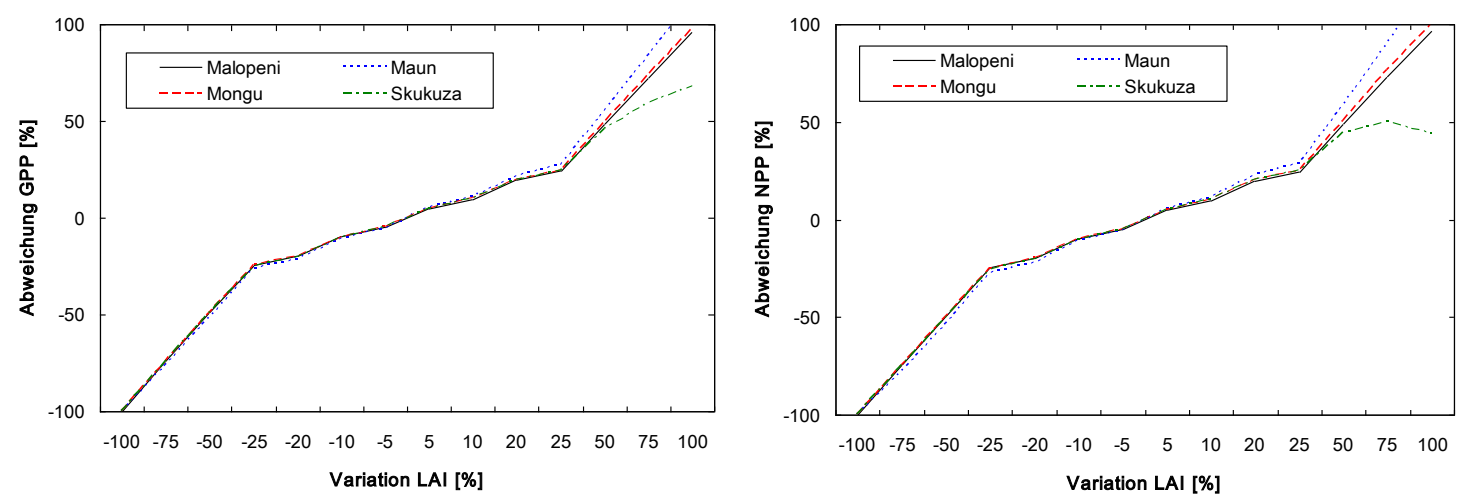

Abbildung 3.2: Mittlere Abweichung der Modellergebnisse GPP (links) und NPP (rechts) vom Referenzwert bei der Variation des Blattflächenindexes.

Entsprechend der hohen Relevanz des Parameters des LAI reagiert das Modell äußerst sensibel auf dessen Variation. Mit Ausnahme der Station Skukuza besteht eine nahezu lineare Abhängigkeit der Modellergebnisse vom Blattflächenindex (zu beachten ist die variierende Schrittweite der Veränderungen). Wie zu erwarten fällt die Produktivität bei einem LAI von null ebenfalls auf null ab und steigt dann linear mit steigendem LAI und erzielt bei verdoppeltem LAI ebenfalls etwa den doppelten Wert. Für die Station Skukuza ist die Steigerung der Produktivität ab einer Erhöhung von mehr als 50\% weniger ausgeprägt. Um diesen Verlauf erklären zu können, muss zusätzlich der maximale Wert des LAI der einzelnen Stationen betrachtet werden. Für die unveränderten Zeitreihen liegt $\Lambda_{\max }$ der Station Skukuza mit $2.9 \mathrm{~m}^{2} \mathrm{~m}^{-2}$ deutlich über dem der anderen Stationen (2.0, 1.3 und 1.8 entsprechend für Malopeni, Maun und Mongu). Bei einer Erhöhung um 50\% erreicht $\Lambda_{\max }$ der Station Skukuza bereits einen Wert von über $4 \mathrm{~m}^{2} \mathrm{~m}^{-2}$, während die restlichen Stationen erst bei maximal $3 \mathrm{~m}^{2} \mathrm{~m}^{-2}$ liegen. In der Modellformulierung wird ab einem LAI von $3 \mathrm{~m}^{2} \mathrm{~m}^{-2}$ ein konstanter Bedeckungsgrad von $f_{c}=0.9$ angenommen (siehe Gleichung (2.15)). Dieser wird für die Berechnung der thermischen Transmissivität $t_{l, v}$ benötigt (Gleichung (2.14)), welche wiederum mit steigender Blattbedeckung abnimmt. Demnach steigt die vom Bestand aufgenommene solare Einstrahlung $R_{n v}$, was die Vegetationstemperatur erhöht. Wird nun eine Bestandstemperatur von über $55^{\circ} \mathrm{C}$ erreicht, fällt die Produktivität durch die in 
Kapitel 2.3.3 (Gleichung (2.47)) beschriebene Limitierung rapide ab. Bei Maximaltemperaturen für die Stationen von etwa $40^{\circ} \mathrm{C}$ ist ein Überschreiten dieses Grenzwertes durchaus realistisch und wird offensichtlich bei dichteren Vegetationsbeständen schnell erreicht.

\subsubsection{Die Temperatur}

Die komplexeste Betrachtung erfordert die Temperatur als Eingangsparameter, da diese in nahezu jedem Bereich berücksichtigt werden muss. Die Verdampfungswärme des Wassers, die Schneedicke, die thermische Strahlung des Bodens (Gleichung (2.13)), der Druck (Gleichung (2.54)) und schließlich die zentralen Parameter der maximalen Carboxylierungsrate (Gleichungen (2.44) bzw. (2.46)), der maximalen Elektronentransportrate (Gleichung (2.43)) sowie der Blattatmung (Gleichung (2.16)) werden abhängig von der Temperatur berechnet. Wie zu erwarten, sinkt die Produktivität der Pflanzen mit fallender Temperatur $\mathrm{ab}$ und steigt zunächst bei höheren Temperaturwerten. Aus Abbildung 3.3 ist der Unterschied zwischen C3- und C4-Mechanismus sehr gut ersichtlich. Trotz niedrigstem LAI-Wert (0.47) erzielt die Station Maun (C4-Grasland) mit ansteigender Temperatur die höchste Produktivität. Dies spiegelt die bessere Anpassung der Pflanzen mit C4Mechanismus an hohe Temperaturen wieder. Insgesamt besitzt der C4-Mechanismus eine stärkere Abhängigkeit von der Temperatur.
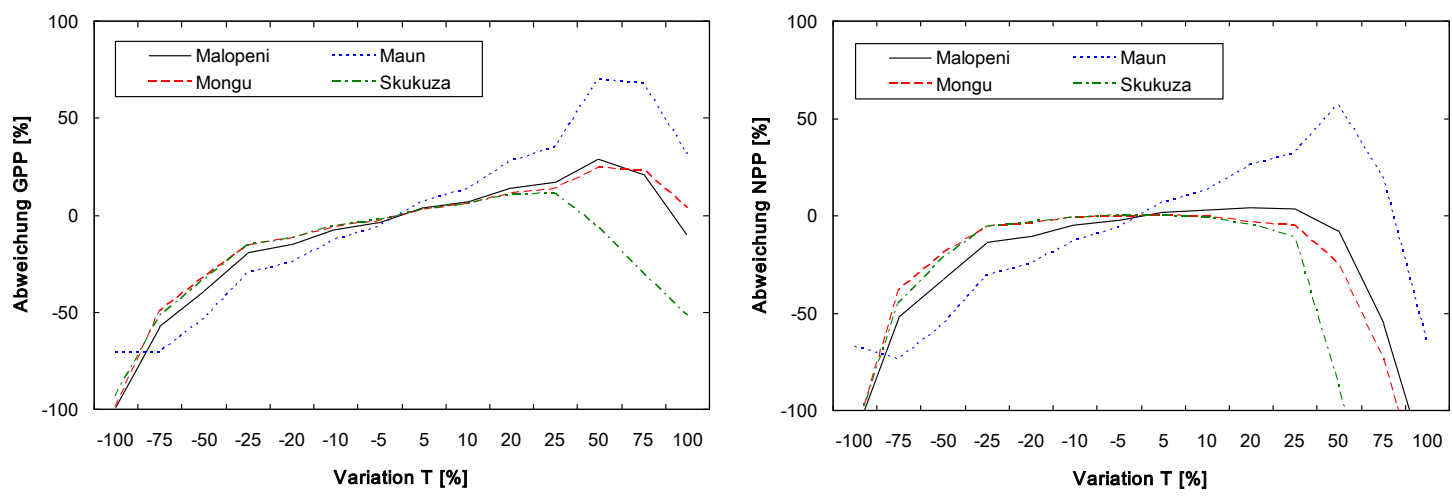

Abbildung 3.3: Mittlere Abweichung der Modellergebnisse GPP (links) und NPP (rechts) vom Referenzwert bei der Variation der Temperatur.

Als wesentlicher Punkt ist die Limitierung der Photosyntheserate bei hohen Bestandstemperaturen zu nennen. Mittels des Limitierungsfaktors $\eta$ (Gleichung (2.47)) wird die Assimilation von Kohlenstoff ab Temperaturen von $55^{\circ} \mathrm{C}$ stark reduziert. Ausgehend von den durchschnittlichen Maximaltemperaturen tritt diese Limitierung bei einer Erhöhung um 94\% für die Station Malopeni, um 86\% für Maun, um 92\% für Mongu und erst bei einer Erhöhung um 120\% für Skukuza in Kraft. Wie aus den Verläufen der relativen Änderung 
der GPP in Abbildung 3.3 ersichtlich ist, wird die Limitierung bereits wesentlich früher erreicht. Dies ist darin begründet, dass die Bestandstemperatur meist höher ist als die Lufttemperatur, wie im vorherigen Kapitel 3.1.2 in Zusammenhang mit dem Blattflächenindex beschrieben. Aus der Kombination von hohem maximalen Blattflächenindex und steigender Temperatur bestimmt sich der Punkt, an dem die Limitierung einsetzt. Die NPP zeigt einen wesentlich stärkeren Rückgang bei entsprechend hohen Temperaturen, da die Erhaltungsatmung, die sich aus der Blattatmung bestimmt (Gleichung (2.50)), ebenfalls stark temperaturabhängig ist, zumal diese Abhängigkeit nichtlinear ist (Abbildung 3.4).

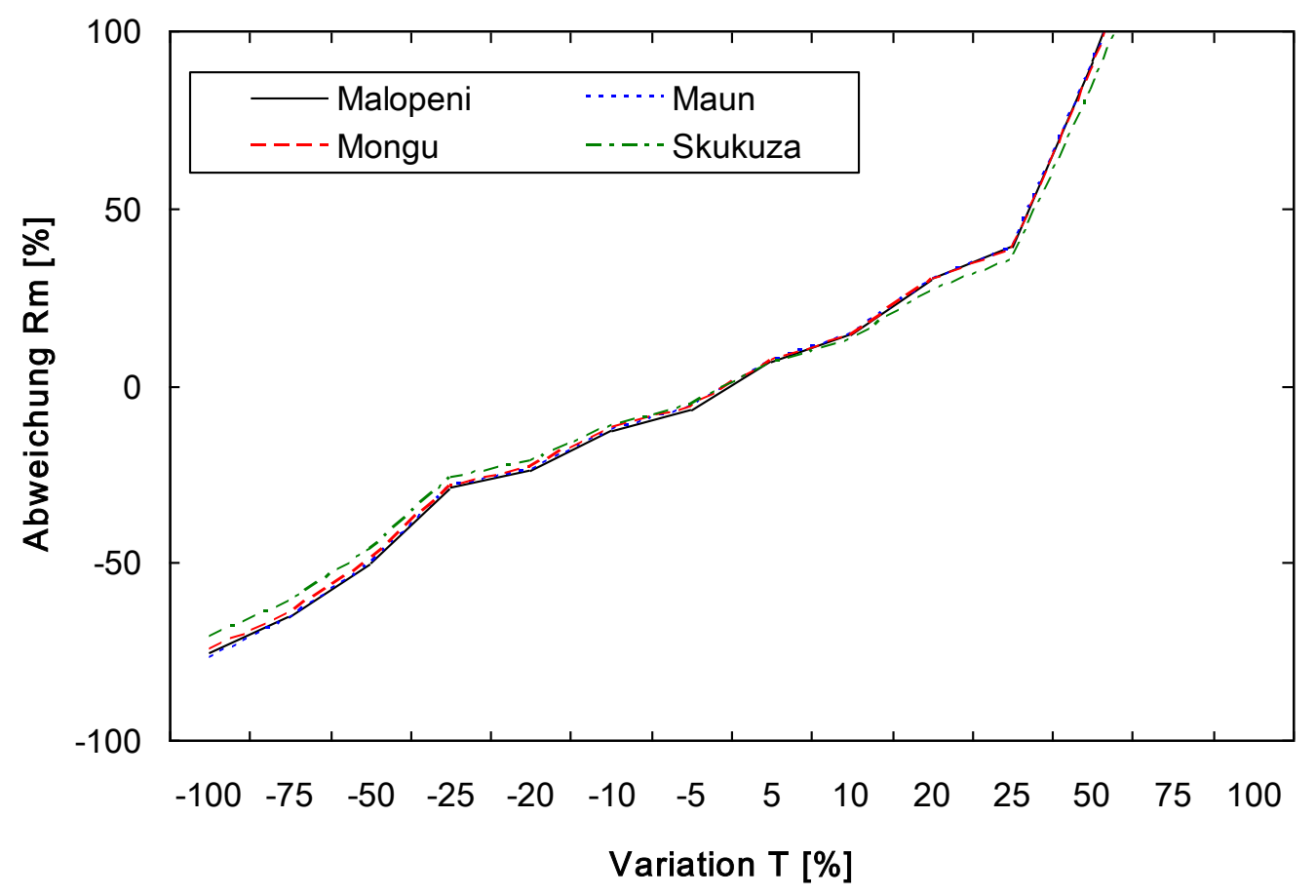

Abbildung 3.4: Mittlere Abweichung der Erhaltungsatmung $R_{m}$ vom Referenzwert bei der Variation der Temperatur.

\subsubsection{Die Windgeschwindigkeit}

Die Windgeschwindigkeit wird bei der Berechnung der aerodynamischen Leitfähigkeit $G_{a}$ (Gleichung (2.25)) benötigt. Über $G_{a}$ wird einerseits die Bestandstemperatur (Gleichung (2.24)) variiert und andererseits die potentielle Rate der Transpiration (Gleichung (2.29)) bestimmt. Wie in Abbildung $3.5 \mathrm{zu}$ sehen ist, sind die Auswirkungen einer veränderten Windgeschwindigkeit eher gering. Nur bei geringen Werten der Windgeschwindigkeit sind nennenswerte Veränderungen von bis zu 15\% zu erkennen. Anscheinend wird im Modell 
ein Wert der Windgeschwindigkeit von exakt $0 \mathrm{~m} \mathrm{~s}^{-1}$ nicht abgefangen, der zunächst zu einer aerodynamischen Leitfähigkeit von $0 \mathrm{~m} \mathrm{~s}^{-1}$ führt. Zur Berechnung der Bestandstemperatur wird durch $G_{a}$ dividiert, was schließlich zu einem ungültigen Wert führt. Da in keinem bisherigen Modelllauf größere Instabilitäten aufgetreten sind, wird angenommen, dass dieser Fall einerseits sehr selten auftritt und andererseits in diesem Ausmaß vernachlässigbare Auswirkungen hat. Für künftige Modellierungen wird $\mathrm{u}=0 \mathrm{~m} \mathrm{~s}^{-1}$ allerdings abgefangen. In dieser Betrachtung wird eine Reduzierung um 100\% nicht berücksichtigt.
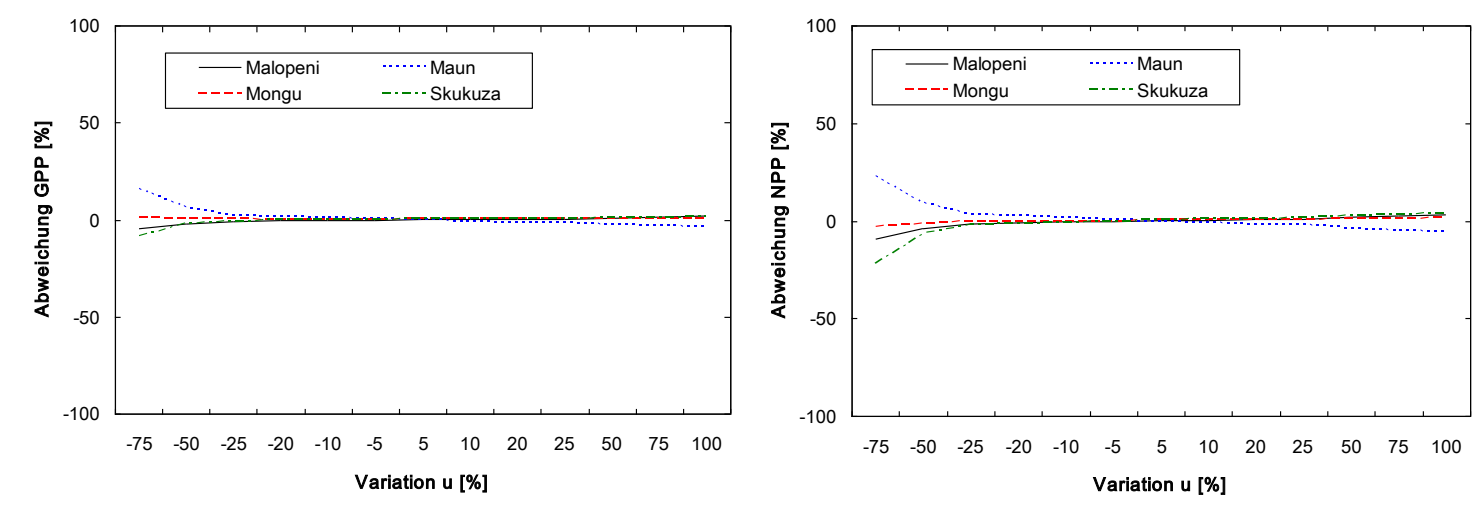

Abbildung 3.5: Mittlere Abweichung der Modellergebnisse GPP (links) und NPP (rechts) vom Referenzwert bei der Variation der Windgeschwindigkeit.

\subsubsection{Der Niederschlag}

Die Sensitivität des Modells auf den Niederschlag beruht im Wesentlichen auf dem Eintrag in den Bodenwasserhaushalt und die Evapotranspiration der Vegetation, speziell dem Gasaustausch bei nasser Blattfläche. In Abbildung 3.6 sind beide Effekte zu erkennen. Der Bodenwasserhaushalt wird einmal täglich berechnet.
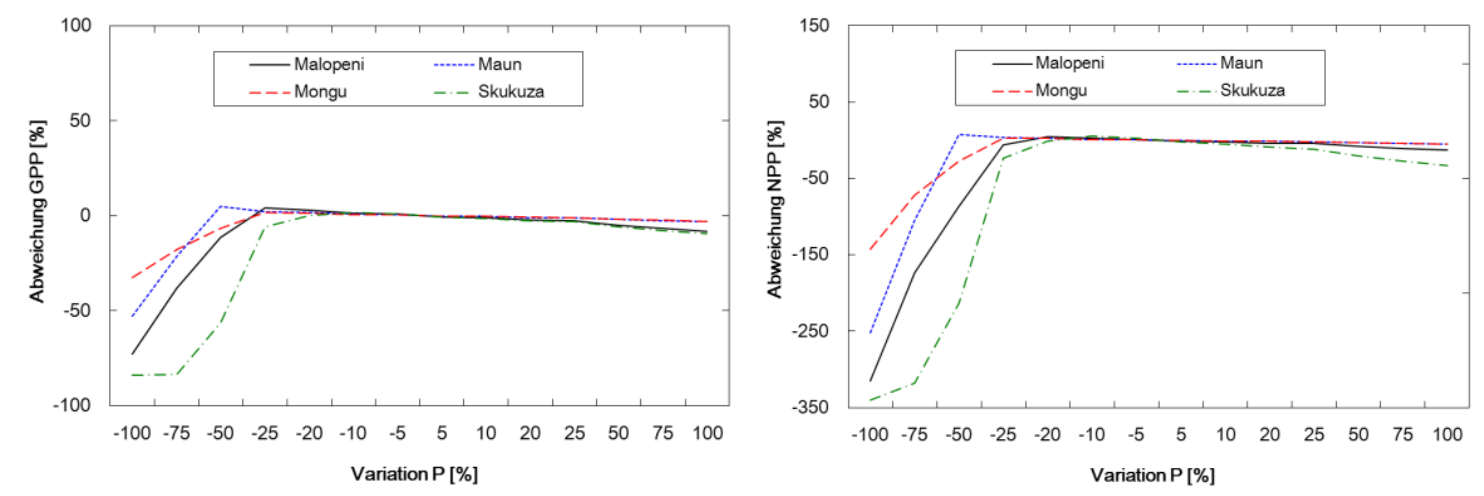

Abbildung 3.6: Mittlere Abweichung der Modellergebnisse GPP (links) und NPP (rechts) vom Referenzwert bei der Variation des Niederschlags. 
In der Regel kann der Boden eine gewisse Menge an Wasser über mehrere Tage ohne zusätzlichen Eintrag durch den Niederschlag speichern. Dies ist abhängig von den speziellen Bodeneigenschaften. Ist diese Speicherfähigkeit gering ausgeprägt, wie beispielsweise bei dem Bodentyp „Eutric Regosol“ der Station Skukuza, trocknet der Boden schneller aus und es ist nicht mehr genug Wasser für die Pflanze verfügbar, um die photosynthetischen Prozesse zu erhalten. Die Vegetation unterliegt dem Trockenstress (vgl. Kapitel 2.4.4) und verringert die Assimilation von Kohlenstoff. Ist der Wassereintrag in den Boden regelmäBig und ausreichend genug, so dass die Pflanze nicht unter Trockenstress gerät, wird die Blattbenetzung ausschlaggebend für die Auswirkungen der Variationen. Wie vorher in Kapitel 2.3.1 beschrieben, tritt Transpiration nur bei trockener Vegetation auf. Über die Transpirationsrate wird der Wasserbedarf, der dem Boden entzogen werden soll, bestimmt. Mit zunehmendem Wasserreservoir auf den Blättern wird demnach die Wassermenge geringer, die dem Boden entzogen wird und der Pflanze zur Verfügung steht. Dadurch verringert sich entsprechend die Produktivität. Diese Verringerung ist allerdings relativ gering.

\subsubsection{Der Bewölkungsgrad}

Der Bewölkungsgrad (1- $\left.r_{P A R}\right)$ wird verwendet, um aus der potentiellen PAR (Gleichung (2.57)) den Anteil zu berechnen, der der Vegetation für die Photosynthese zur Verfügung steht. Daraus ergibt sich die Annahme, dass mit steigender Bewölkung die Produktivität der Vegetation abnimmt. Dies ist auch aus Abbildung 3.7 abzulesen. Bei komplett bewölktem Himmel sinkt die Produktivität auf null ab. Wird der Himmel zunehmend wolkenfrei, steigt die Produktivität zunächst linear an.
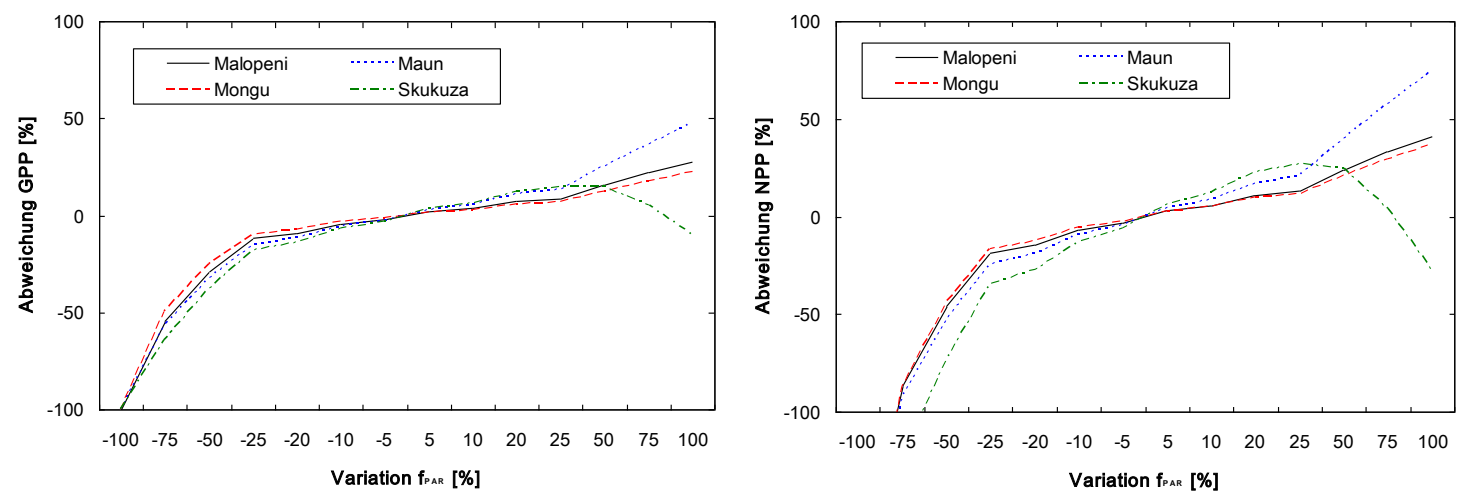

Abbildung 3.7: Mittlere Abweichung der Modellergebnisse GPP (links) und NPP (rechts) vom Referenzwert bei der Variation des Bewölkungsgrades.

Eine Besonderheit stellt erneut die Station Skukuza dar, was sich allerdings wie bei der bisherigen Betrachtung auch auf die Limitierung durch die erhöhte Bestandstemperatur 
zurückführen lässt, in die der Bewölkungsgrad über die PAR und schließlich die Strahlungsbilanz $\left(R_{n v}\right)$ eingeht (siehe Gleichung (2.24)). Bei zunehmend freiem Himmel erhöht sich die PAR und damit die Strahlungsmenge $R_{n v}$, die bei der Vegetation ankommt. Dies erhöht schließlich die Bestandstemperatur der Vegetation, wodurch bei hoher Vegetationsdichte, wie im Falle der Station Skukuza, schnell die kritische Temperatur von $55{ }^{\circ} \mathrm{C}$ erreicht wird.

\subsubsection{Die Landbedeckungsklassifikation}

Über die Zuweisung spezifischer Landnutzungs- und Landbedeckungsklassen werden die für die Beschreibung der Photosynthesereaktionen maßgeblichen Parameter der Elektronentransportrate $J_{\max }$ und der Carboxylierungsrate $V_{c \text {, } \max }$ festgelegt. Dadurch wird unterschieden, wie gut die Assimilation von Kohlenstoff bei den jeweiligen Pflanzenarten ausgeprägt ist.
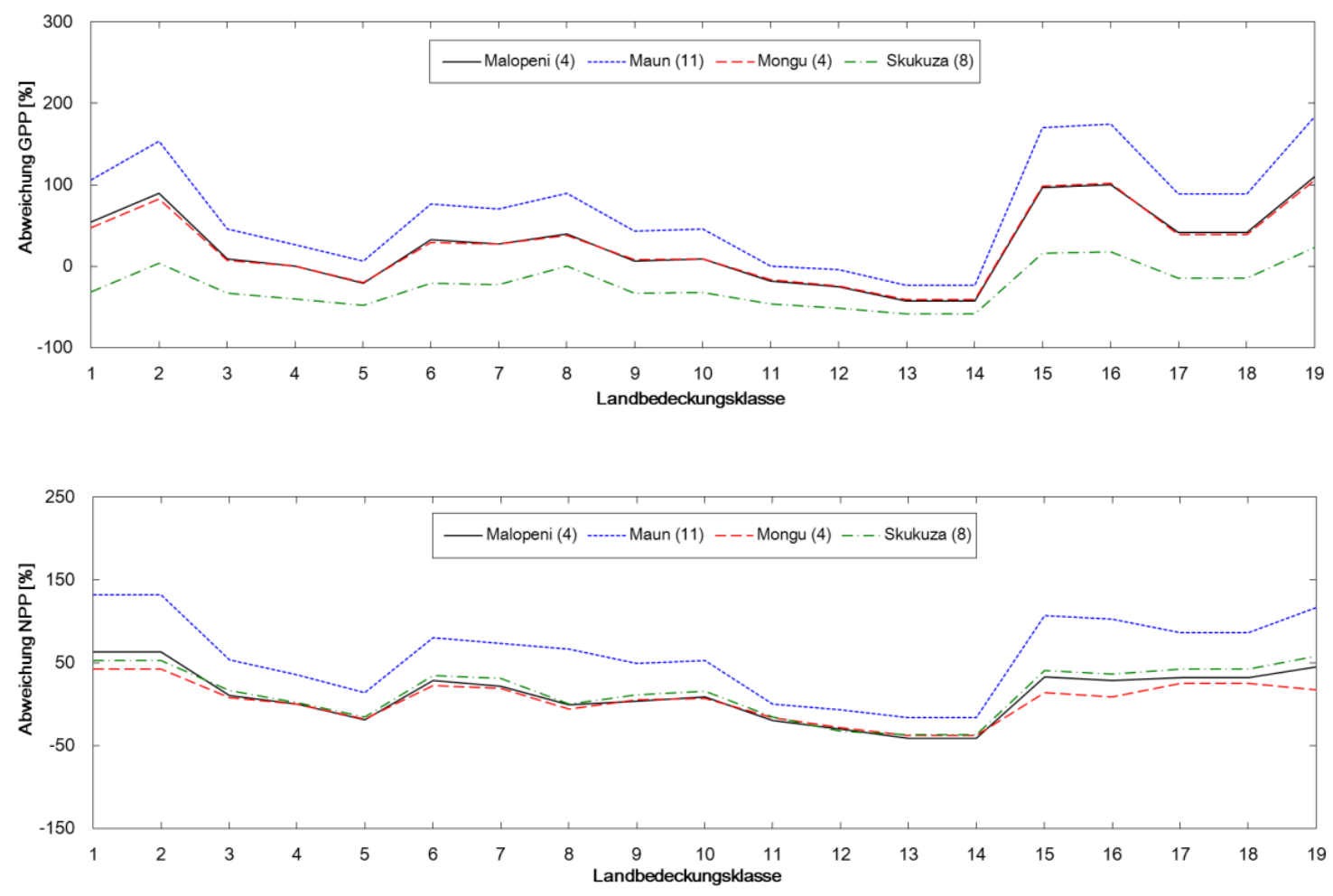

Abbildung 3.8: Mittlere Abweichung der Modellergebnisse GPP (oben) und NPP (unten) vom Referenzwert bei Veränderung der Vegetationsklassifikation entsprechend der in Tabelle 2.4 definierten Vegetationstypen.

Demnach ist zu erwarten, dass hier die höchsten Abweichungen gegenüber dem Referenzwert von GPP und NPP auftreten. Aus Gründen der Übersichtlichkeit wurde nur zwischen den Klassen 1 (immergrüner, tropischer Laubwald) bis 19 (sommergrüne, gemäßigte 
Baumfrüchte) variiert (siehe Tabelle 2.4). Die Abweichungen zeigen sich anhand von Abbildung 3.8 sehr eindeutig, wobei hier Differenzen von bis zu knapp 200\% der GPP und bis zu 130\% der NPP beobachtet werden können. Die größten Differenzen ergeben sich entsprechend den größten Unterschieden in den Parametern $J_{\max }$ und $V_{c, \max }$. Diese starke Abhängigkeit konnte auch im Rahmen einer separaten Studie bei der Variation der Parameter $V_{c, \max }$ und $J_{\max }$ selbst gezeigt werden. Dabei wurde jeweils ein Parameter festgehalten, während der andere verändert wurde. Betrachtet wurde nur das Ergebnis der NPP, die GPP wurde nicht berücksichtigt. Für die maximale Carboxylierungsrate $V_{c, \max }$ ergibt sich ein linearer, negativer Zusammenhang, der sich direkt aus der Abhängigkeit der Photorespiration $R_{d}$ herleiten lässt. Für $J_{\max }$-Werte $<100 \mu \mathrm{mol}\left(\mathrm{CO}_{2}\right) \mathrm{m}^{-1} \mathrm{~s}^{-1}$ bewirkt eine Erhöhung der Elektronentransportrate dagegen einen starken Anstieg der NPP. Dieser geht zwischen 200 und $300 \mu \mathrm{mol}\left(\mathrm{CO}_{2}\right) \mathrm{m}^{-1} \mathrm{~s}^{-1}$ in Sättigung, was dem Verlauf von Gleichung 2.20 entspricht. Der Verlauf der GPP wird primär von dem der Elektronentransportrate geprägt. Eine Ausnahme bildet die C4-Vegetation der Klassen 11 und 12 mit den sehr geringen $V_{c, \text { max }}$-Werten von $8 \mu \mathrm{mol}\left(\mathrm{CO}_{2}\right) \mathrm{m}^{-1} \mathrm{~s}^{-1}$, die in diesem Falle nicht dem Verlauf von $J_{\max }$ folgt. Entsprechendes gilt auch für die resultierende Variation in der NPP. Hier wird die Abhängigkeit des Ergebnisses von der Elektronentransportrate durch den größeren Einfluss der maximalen Carboxylierungsrate über die berücksichtigte autotrophe Respiration $R_{a}$ verringert. Die Variationen fallen dementsprechend geringer aus als bei der GPP, sind mit teilweise über $100 \%$ allerdings noch immer unter den größten in dieser Sensitivitätsstudie.

Mit der Sensitivitätsstudie konnten die wesentlichen Eingangsparameter identifiziert werden, die das Modellergebnis signifikant beeinflussen. Dabei muss zwischen den Ergebnissen der GPP und der NPP unterschieden werden, da bei dem letzten Rechengang auch einige Parameter, wie die Temperatur, die PAR oder die maximale Carboxylierungsrate eine wichtige Rolle spielen. Den größten Einfluss hat erwartungsgemäß eine Änderung des Vegetationstyps, mit den relevanten Parametern für die Effektivität der Photosynthese. Die GPP erfährt Variationen von bis zu etwa $200 \%$ bei einer Fehlklassifizierung von Buschoder Ackerland als Grasland. Auch die Verfügbarkeit an photosynthetisch aktiver Strahlung hat sehr großen Einfluss auf das Modellergebnis und kann im Falle der NPP bei einem Fehler von $50 \%$ bereits eine Abweichung von bis zu 150\% bewirken.

Hohe Fehler im Datensatz der Temperatur haben in den hier betrachteten warmen Gebieten häufiger eine Verringerung von GPP und NPP zur Folge, egal ob die Temperatur unteroder überschätzt wird. Niedrige Temperaturen verringern generell die photosynthetische Aktivität, bei zu hohen Temperaturen hingegen wird diese durch eine Limitierung reduziert. Ähnliches gilt für den Niederschlag, da eine Erhöhung der Niederschlagsmenge nur zu einer Erhöhung der Produktivität führt, wenn ein Gebiet von Trockenstress betroffen ist. 
$\mathrm{Zu}$ geringer Niederschlag führt zu einem Austrocknen des Bodens, wenn dieser über unzureichende Speicherfähigkeiten verfügt. Kann der Boden dagegen ausreichend lange Wasser speichern, ist die effektive Menge des Bodenwasserreservoirs wenig ausschlaggebend, solange Wasser verfügbar bleibt. Dagegen wird bei erhöhten Niederschlagsmengen die Photosyntheseaktivität durch das auf den Blättern gespeicherte Wasser limitiert.

Im Hinblick auf die geringe Korrelation von Stationswerten und Werten des ECMWF für Tageswerte und den verbesserten Ergebnissen beim Vergleich von Monatssummen des Niederschlags, bestätigt das Ergebnis der Sensitivitätsstudie, dass für das Modell keine tagesgenauen Werte der Niederschlagsmengen benötigt werden, sofern der Eintrag in den Bodenwasserhaushalt über einen längeren Zeitraum eine bessere Qualität aufweist.

Kein Unterschied zwischen den Variationen von GPP und NPP ist für die Änderung der LAI Werte zu erkennen. Diese liegen einheitlich im Bereich von $\pm 100 \%$. Dabei wird ein linearer Zusammenhang zwischen LAI und Produktivität deutlich. Letztlich konnten auch zwei Parameter ausgemacht werden, die keine große Rolle für die Modellierung spielen. Diese sind die Höhe über dem Meeresspiegel bzw. der Luftdruck und die Windgeschwindigkeit. Selbst Variationen der Größen von 100\% hatten bei GPP und NPP keine größeren Änderungen als $\pm 20 \%$ zur Folge. Im Falle der Höhe gingen die Änderungen nicht über 3\% hinaus. Dies sind zudem Maximalwerte die bei der maximalen Variation der Parameter auftreten. Bei geringeren Variationen von Höhe und Windgeschwindigkeit bewegen sich die Änderungen der Ergebnisse bei Werten unter 2\%. Die Qualität des ECMWFDatensatzes der Windgeschwindigkeit wurde in Kapitel 2.4.1.3 als eine der Schlechtesten der ECMWF Daten aufgeführt $(\mathrm{r}=0.47)$. In Anbetracht der gezeigten geringen Auswirkungen des Datensatzes auf das Modellergebnis ist die schlechte Qualität allerdings vertretbar.

Wichtiger hingegen sind die hohen Werte der Korrelationen der für das Modell relevanten Größen, wie Temperatur ( $r=0.9$ ) oder PAR ( $r=0.9)$. Für den LAI oder die Klassifizierung der Vegetationstypen kann für das Arbeitsgebiet leider keine entsprechende Aussage über die Qualität getroffen werden. Daher wird für diese Parameter der jeweilige Wert für das globale Produkt angenommen (LAI (siehe Kapitel 2.4.2): r=0.9, RMSE=0.88; GLC2000 (Mayaux, 2006): Genauigkeit 69\%). Damit kann festgestellt werden, dass das Modell BETHY/DLR mit der guten Qualität der sensiblen Parameter im vorgestellten Arbeitsgebiet auf einer guten Datenbasis aufbaut. Dies ist erforderlich für die Einschätzung der Modellergebnisse und deren Qualität im folgenden Kapitel. Ein Vergleich der Modellergebnisse mit anderen Datensätzen kann damit Aufschluss über die Güte der Modellformulierung selbst geben. Dies soll wesentlicher Bestandteil der weiteren Betrachtungen sein. 



\section{$4 \quad$ Die Modellergebnisse}

Nach der Beschreibung des Modells BETHY/DLR, der Betrachtung der Qualität der Eingangsdaten und der Sensibilität des Modells auf Variationen der Eingangsdaten bei der Anwendung auf das Anwendungsgebiet, sollen nun die Ergebnisse des Modelllaufes analysiert werden. Die im Rahmen dieser Arbeit relevanten Größen sind die Brutto- und die Nettoprimärproduktion, GPP und NPP. Diese werden in täglichen Zeitschritten für das in Kapitel 2.2 definierte Arbeitsgebiet des südlichen Afrika über den Zeitraum von 1999 bis 2010 berechnet. Somit steht eine kontinuierliche Datenreihe über 12 Jahre für die Bewertung der Landdegradation in den Trockengebieten des südlichen Afrika zur Verfügung. Bevor die Zeitreihen allerdings zu diesem Zweck weiter verarbeitet werden können, muss versucht werden, die Güte der Modellergebnisse durch Validierung und Vergleich mit anderen Datensätzen zu bestimmen.

Für eine direkte Validierung des Modells wird die Variation der stehenden Biomasse aus Bodenmessungen über einen bestimmten Zeitraum mit der modellierten Produktivität innerhalb dieses Zeitraumes verglichen. Dazu wird eine Messreihe über acht Jahre für das gesamte Gebiet des Krüger-Nationalparks in Südafrika verwendet. Weiterhin werden die Modellergebnisse an einzelnen Stationen mit Messungen des Kohlenstoffaustausches durch die Eddy-Kovarianz- (EK) Methode verglichen. Dabei werden positive und negative Kohlenstoffflüsse zwischen Ökosystem und Atmosphäre bestimmt, aus denen sich die GPP berechnen lässt. Innerhalb des Arbeitsgebietes stehen, wie bereits erwähnt, vier solcher Messtürme, an denen für unterschiedliche Zeiträume EK-Messungen durchgeführt wurden. Schließlich werden auch Ergebnisse anderer Modelle mit den Ergebnissen von BETHY/DLR verglichen. Dafür konnten für das Arbeitsgebiet globale Datensätze der NPP und GPP, abgeleitet aus AVHRR- und MODIS-Daten, für unterschiedliche Zeiträume verwendet werden.

\subsection{Biomassemessungen im Krüger-Nationalpark}

Seit dem Jahr 1989 erfolgt im Krüger-Nationalpark eine jährliche Begutachtung des Zustandes der Grasvegetation. Dies dient der Kontrolle der Auswirkungen von Brandereignissen und Abgrasung auf die Vegetation (Trollope und Potgieter, 1986; Trollope, 1990). Verschiedene Landschaftstypen werden dabei auf etwa 533 Messgebiete, proportional zu deren Flächenanteilen innerhalb des Parks, aufgeteilt. 


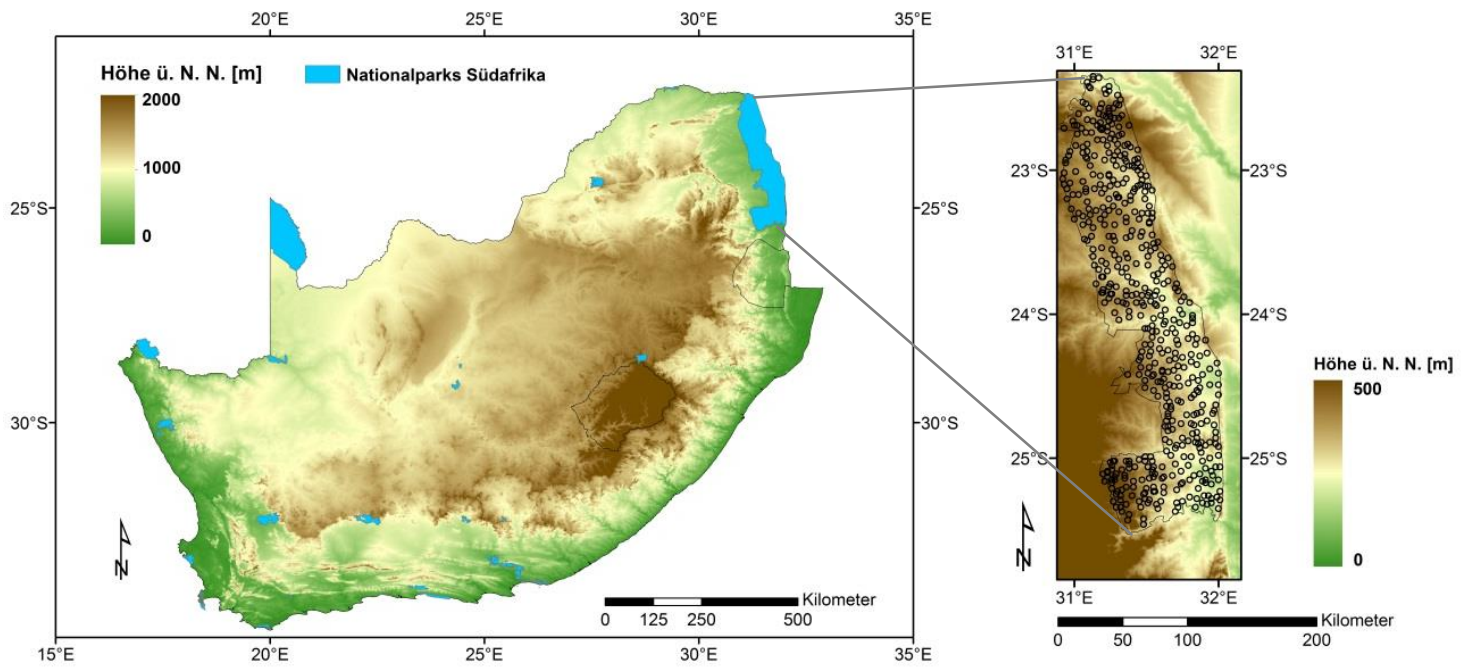

Abbildung 4.1: Lage des Krüger-Nationalparks in Südafrika und Verteilung der Messgebiete innerhalb des Parks. Die Farbskala zeigt die Höhe über Normalnull, links sind die Nationalparks in blau angezeigt, rechts die Orte der Messgebiete als offene Kreise.

Jedes Gebiet umfasst $50 \mathrm{~m}$ x $60 \mathrm{~m}\left(0.003 \mathrm{~km}^{2}\right)$, wobei die Vegetation innerhalb der Gebiete als repräsentativ für die größere Umgebung angenommen wird. Es werden 100 Messungen krautartiger Biomasse in Abständen von $2 \mathrm{~m}$ entlang von Transekten von $50 \mathrm{~m}$ durchgeführt. Mit Hilfe eines Bestandeshöhenmessgerätes (disk pasture meter, DPM) wird die Höhe $h$ der Vegetation an den einzelnen Messpunkten mit einer Genauigkeit von $0.5 \mathrm{~cm}$ gemessen. Das DPM besteht aus einem soliden Messstab über den eine Scheibe mit einem Durchmesser von etwa $50 \mathrm{~cm}$ geschoben wird. An den Messpunkten wird diese Scheibe auf die Vegetation fallen gelassen und die Höhe der Scheibe, die auf der Vegetation zum Liegen kommt, abgelesen. Über gravimetrische Methoden wird zunächst der Wassergehalt der Vegetation ermittelt. Zur Ermittlung der stehenden Biomasse wird schließlich eine Regressionsgleichung bestimmt, die $89.5 \%$ der Variationen der Grasarten in den verschiedenen Landschaftstypen beschreibt (Trollope and Potgieter, 1986):

$$
B=-3019+2260 \sqrt{h}
$$

Die Erhebungen erfolgen jeweils zwischen Ende März und Mitte April. Gestartet wird dann, wenn die krautartige Vegetation beginnt auszutrocknen. Damit wurde der jährliche Bestand an stehender Biomasse in den Jahren 1999 bis 2006 aufgenommen. Die Differenz der gemessenen Biomasse zwischen zwei Jahren wird mit dem akkumulierten, berechneten Zuwachs aus dem Modell für den gleichen Zeitraum verglichen. Die erhobenen Messungen hatten den Schwerpunkt auf Gras und krautartige Vegetation, weswegen im Modell das gesamte Gebiet für den Vergleich als Grasland definiert wird. Die untersuchten Hauptgrasarten betreiben die Photosynthese ausschließlich über den C4-Mechanismus. Daher wurde 
die entsprechende Formulierung ebenfalls für das gesamte Gebiet des Nationalparks gewählt. Leider standen keine Informationen über Sekundärvegetation und den Bedeckungsgrad der Messgebiete zur Verfügung. Die Gewichtung wurde daher zu 100\% auf die Primärklasse verteilt. In der Regel wird der Bedeckungsgrad im Savannengebiet des KrügerNationalparks wesentlich geringer sein. In Ermangelung genauer Daten wird demnach eine systematische Überschätzung der Produktivität durch das Modell erwartet. Weiterhin werden im Modell äußere Einflüsse, wie das Abbrennen oder Abgrasen der Vegetation nicht berücksichtigt. Um dies ansatzweise aus den Messdaten herauszufiltern, werden negative Differenzen von dem Vergleich ausgenommen.

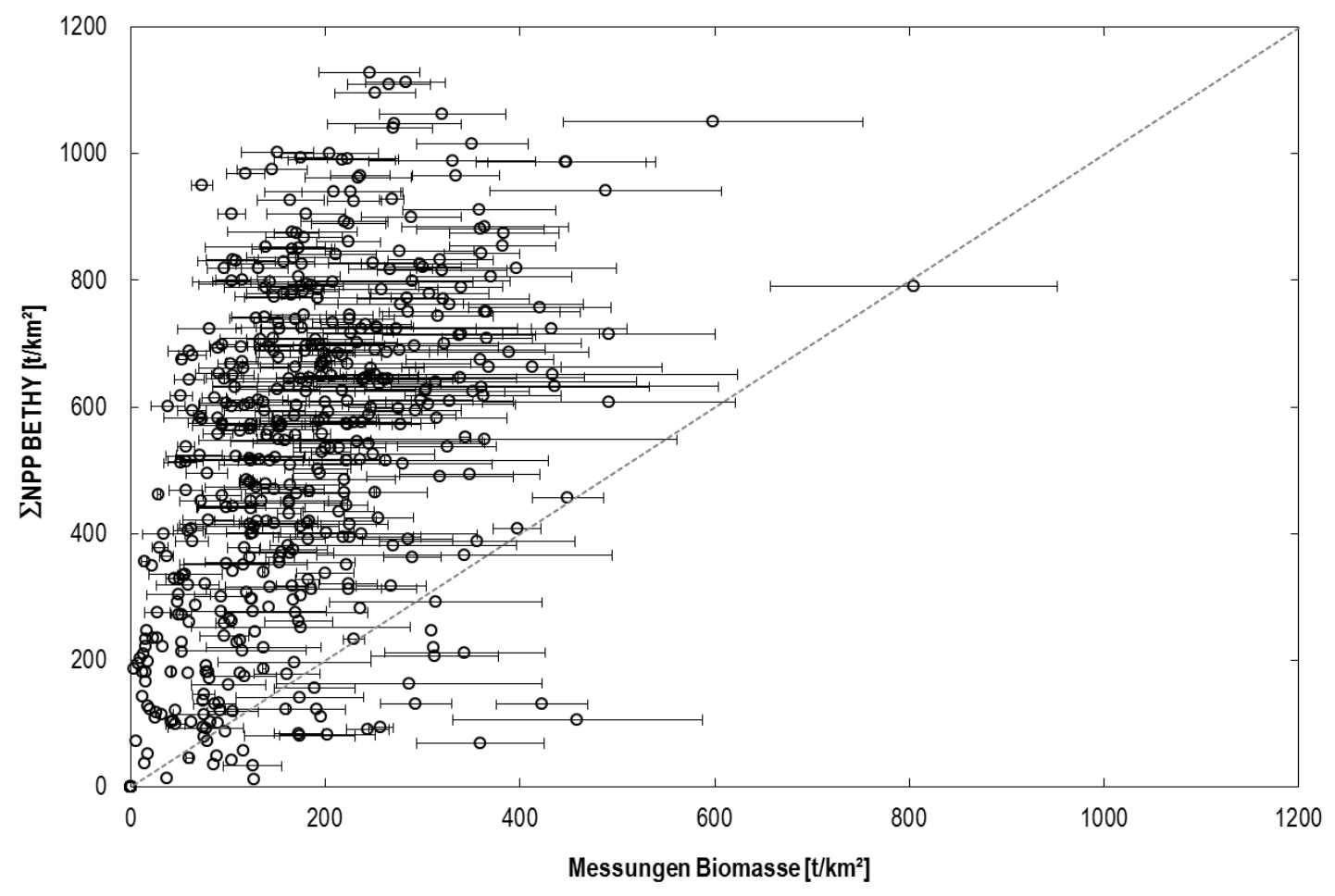

Abbildung 4.2: Streudiagramm der akkumulierten NPP aus dem Modell und den Differenzen der Biomassemessungen im Krüger-Nationalpark. Die Fehlerbalken zeigen die Standardabweichung der Messdaten an. Die gestrichelte Linie markiert die 1:1-Gerade.

Abbildung 4.2 zeigt den Vergleich der jährlich aufsummierten NPP des Modells über den gesamten Zeitraum mit den entsprechend aufsummierten jährlichen Differenzen der Biomassemessungen. Daraus kann eine deutliche Überschätzung des Zuwachses durch das Modell gegenüber den Messwerten abgelesen werden, die mit einem Faktor von $1.5 \mathrm{zu}$ höheren Werten hin zunimmt. Die Korrelation ergibt sich aus den Summen aller Stationen zu $r=0.57$. Allerdings muss auch die hohe Variation in den Messdaten berücksichtigt werden, die durch die Standardabweichung als Fehlerbalken gezeigt ist. Die Überschätzung 
sowie die geringe Korrelation kann darauf zurückgeführt werden, dass die bereits angesprochenen äußeren Einflüsse nicht in das Modell mit einfließen.

Ein Hauptbeweggrund zur Durchführung der jährlichen Bewertung des Vegetationszustandes ist die Aufnahme der jährlich abgebrannten Vegetation. Demnach ist nicht auszuschließen, dass trotz der Vernachlässigung negativer Jahresdifferenzen auch geringe Zuwachsraten die Folge von Feuerereignissen oder Abgrasung sind. Diese verursachen wiederum die sichtbare Variabilität der gemessenen Biomasse.

In Abbildung 4.3a sind die Korrelationskoeffizienten der einzelnen Stationen und die Verteilung der betrachteten Stationen dargestellt. Nach Vernachlässigung der negativen Vegetationstrends und Stationen, für die weniger als vier Messpunkte zur Verfügung standen, reduzierte sich die Anzahl der Messgebiete von anfänglich 533 auf nur noch 236. Die Variation in den Korrelationen zwischen den Messwerten und Modellwerten ist sehr hoch, was auch aus der Häufigkeitsverteilung in Abbildung 4.3b zu entnehmen ist.

(a)

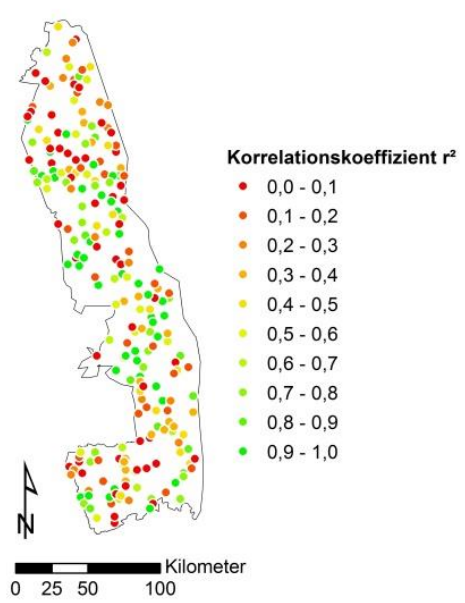

(b)

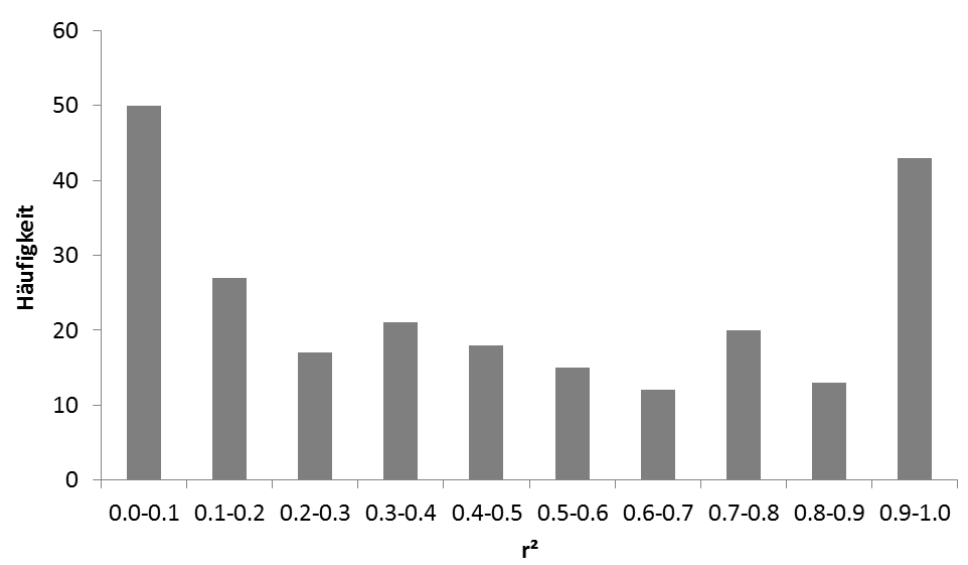

Abbildung 4.3: Korrelationskoeffizient der einzelnen Stationen zwischen den Messungen der Biomasse und den modellierten NPP-Summen über die Wachstumsphase.

Auch wenn viele Messpunkte entweder eine besonders gute (0.9-1.0) oder eine besonders schlechte (0.0-0.1) Korrelation zeigen, ist der Hauptteil auf die übrigen Bereiche dazwischen verteilt, so dass keine eindeutige Häufung ausgemacht werden kann. Die mittlere Korrelation aus den Vergleichen der einzelnen Gebiete beträgt $r=0.69$, wobei die Modellwerte meist über denen der Messungen liegen. Ein Kritikpunkt ist sicherlich die kurze Zeitserie der gemessenen Daten (maximal sieben Wachstumsphasen), die hier nur betrachtet werden kann. Durch die geringe Anzahl an Vergleichswerten wird das Ergebnis wesentlich durch den Einfluss von Ausreißern in den Daten beeinflusst, die nicht vollständig aus- 
geschlossen werden können. Der Einfluss des Skalenunterschiedes der beiden Datensätze wird als gering eingeschätzt, da die Messgebiete als repräsentativ für das umgebende Land gelten. Demnach sollten auch mögliche Abweichungen in den Projektionen von Messdaten und Modellergebnissen in der zur Analyse verwendeten GIS-Software vernachlässigbar sein.

Angesichts der Einschränkungen stellt sich die Frage, wie aussagekräftig der Vergleich der Modellergebnisse mit den gemessenen Biomassewerten ist. Zunächst kann die systematische Überschätzung der Produktivität durch das Modell über den nicht berücksichtigten Bedeckungsgrad erklärt werden. In Anbetracht der fehlenden Informationen über Brandereignisse, Einfluss der Abweidung durch Tiere und den Bedeckungsgrad sowie der kurzen Zeitspanne des Vergleiches sind die Ergebnisse dennoch zufriedenstellend. So kann zwar keine hohe Korrelation zwischen den Messwerten und den Modellergebnissen ermittelt, aber zumindest ein Trend festgestellt werden. Bessere Ergebnisse werden von einem Vergleich über einen längeren Zeitraum erwartet. Dazu müsste allerdings die Datengrundlage des Modells angepasst werden. Wie in Kapitel 2.4.2 beschrieben, ist der Modellierungszeitraum von 1999 bis 2010 durch den SPOT-VGT-LAI-Datensatz bestimmt. Die Erhebungen zur Biomasse im Krüger-Nationalpark gehen bis auf das Jahr 1989 zurück. Für einen längerfristigen Vergleich könnte aus den für diesen Zeitraum verfügbaren AVHRRNDVI-Zeitserien der LAI als Eingangsparameter abgeleitet werden. 


\subsection{Messungen des Kohlenstoffflusses durch Eddy-Kovarianz-Türme}

Eine weitere Möglichkeit der Evaluierung der Modellergebnisse stellt der Vergleich mit Messungen durch Eddy-Kovarianz-Türme dar. Dabei werden vertikale turbulente Flüsse an der Grenzschicht zwischen Biosphäre und Atmosphäre gemessen. Zum einen wird die Windgeschwindigkeit durch ein Ultraschallanemometer, zum anderen die Konzentration von Spurengasen durch einen Infrarot-Gasanalysator, darunter auch die Konzentration von Kohlenstoffdioxid, gemessen. Die Messgeräte werden an der Spitze eines Turmes oder Mastes direkt über dem Vegetationsbestand angebracht (Abbildung 4.4). Aus den Messungen der aufwärts und abwärts gerichteten Stoffflüsse zu verschiedenen Zeitpunkten kann der effektive Stoffaustausch an dieser Grenzschicht innerhalb dieses Zeitraumes bestimmt werden. Mathematisch lässt sich die Berechnung als die Kovarianz zwischen der Abweichung der Windgeschwindigkeit vom Mittelwert und der Abweichung der Stoffkonzentration vom Mittelwert ausdrücken, multipliziert mit dem mittleren Luftdruck (Baldocchi et al., 1988). Der daraus bestimmte Fluss von Kohlendioxid zwischen Vegetation und Atmosphäre wird als Nettoökosystemaustausch (engl. Net Ecosystem Exchange, NEE) bezeichnet. Dabei werden Flüsse aus der Atmosphäre in die Vegetation als negativ, der Austausch aus der Vegetation heraus als positiv definiert. Die NEE wird nach Reichstein et al. (2005) weiter in die Atmung des Ökosystems $R_{\text {eco }}$ und die Bruttoökosystemproduktivität (engl.: Gross Ecosystem Exchange, GEP) aufgeteilt:

$$
N E E=R_{e c o}-G E P
$$

Nachts ist der Beitrag der GEP gleich null und der $\mathrm{CO}_{2}$-Fluss vom Ökosystem in die Atmosphäre gleicht $R_{\text {eco }}$. Tagsüber hat die GEP den größten Anteil und die NEE ist meist negativ. Um die beiden Beiträge zu trennen werden die nächtlichen Messungen der Ökosystematmung über die Temperatur für den Tagesverlauf unter Verwendung des Regressionsmodells nach Lloyd und Taylor (1994) interpoliert:

$$
R_{e c o}(t)=R_{r e f}(t) e^{E_{0}\left(\frac{1}{T_{r e f}-T_{0}}-\frac{1}{T_{\text {Boden }}(t)-T_{0}}\right)}
$$

mit dem konstanten Regressionsparameter $T_{0}=-46.02{ }^{\circ} \mathrm{C}$, der Referenztemperatur $T_{\text {ref }}=10$ ${ }^{\circ} \mathrm{C}$, der Bodentemperatur $T_{\text {Boden }}$ und dem temperaturunabhängigen Beitrag der Atmung $R_{\text {ref }}$. $E_{0}$ ist der freie Parameter der Aktivierungsenergie zur Bestimmung der Temperaturabhängigkeit. Eine genaue Beschreibung der Methode findet sich in Reichstein et al. (2005). Aus der Differenz der interpolierten Ökosystemrespiration und des gemessenen Nettoökosystemaustausches ergibt sich demnach der in dem Ökosystem aufgenommene Anteil des Kohlendioxids in Form der GEP. Wichtig ist, den Unterschied zwischen der hier abgeleite- 
ten GEP und der modellierten GPP zu beachten. Die GEP berücksichtigt beispielsweise nicht die Weiterverwendung von Kohlendioxid während der Dunkelreaktionen, bei der die in der Lichtreaktion gewonnenen ATP und NADPH verbraucht werden (Stoy et al., 2006). Andererseits umfassen die Messungen der Ökosystemrespiration auch andere Kohlenstoffquellen wie Brände, Einflüsse der Fauna sowie anthropogene Ursachen, die im Modell nicht parametrisiert sind. Dennoch wird über längere Zeiträume ein vergleichbarer Verlauf erwartet, da die maßgeblichen Parameter in beiden Betrachtungen berücksichtigt werden.
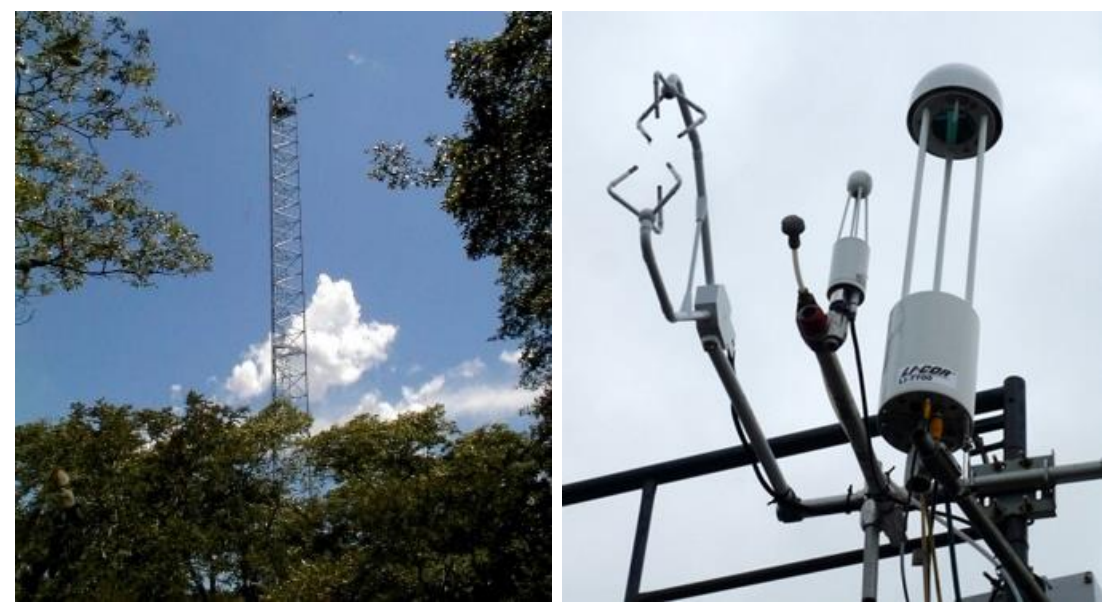

Abbildung 4.4: Messturm der Station Mongu (links) und die Anordnung der Messgeräte an der Spitze des Messturms (rechts), mit Ultraschallanemometer (rechtes Bild links), Infrarot Gasanalysator für $\mathrm{CO}_{2}$ und $\mathrm{H}_{2} \mathrm{O}$ (rechtes Bild Mitte) und Gasanalysator für $\mathrm{CH}_{4}$ (rechtes Bild rechts). (Bilder: fluxnet.ornl.gov und licor.com)

Innerhalb des Arbeitsgebietes befinden sich vier solcher Messstationen, die in unterschiedlichen Zeitabschnitten aber noch innerhalb des Modellierungszeitraumes betrieben wurden (Abbildung 4.5). Die nördlichste Station, Mongu, liegt in Sambia, in der Westprovinz, im Südwesten des Landes. Die Vegetation wird als Miombo Savanne bezeichnet, gemäß der regionalen Bezeichnung der häufigsten Baumarten, die auch den primären Bewuchs als geschlossener Laubwald bestimmen (Campbell, 1996). Die sekundäre Vegetation reicht von dichten Grasbüscheln bis zu spärlicher Bedeckung mit Kräutern und kurzen Gräsern. Der Zeitraum der Messungen erstreckt sich über 23 Monate, von September 2007 bis Juli 2009. Im Nord-West-Distrikt von Botsuana liegt die zweite Station, Maun, deren Landbedeckung als Mopane Savanne bezeichnet wird, ebenfalls nach der Hauptbaumart. Der sommergrüne Laubbaum tritt als offener Wald mit weiten grasbedeckten Flächen auf (Cowling et al., 1997). Die Messungen wurden von März 1999 bis Oktober 2001 durchgeführt. Die beiden Stationen Malopeni und Skukuza befinden sich im Krüger-Nationalpark in Südafrika. Der Nordteil des Parks, in dem sich die Station Malopeni befindet, ist ebenfalls von offenen Mopanewäldern mit spärlichem Grasbewuchs als sekundäre Vegetation 
geprägt (Cowling et al., 1997). Diese Station wurde nur für zehn Monate von März 2009 bis Dezember 2009 betrieben. Das südwestliche Gebiet um Skukuza beheimatet offene Akazienwälder mit freien, vereinzelt grasbewachsenen Flächen (Landmann, 2003).

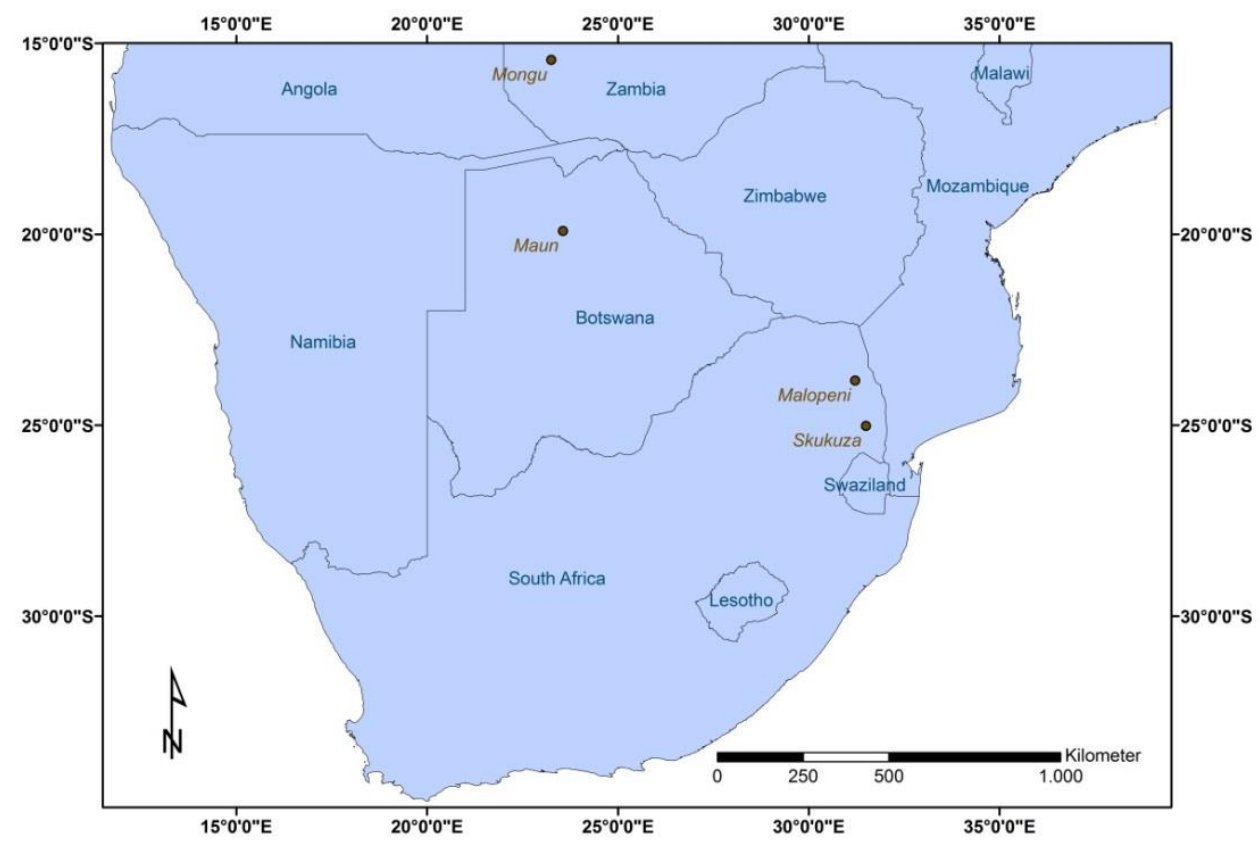

Abbildung 4.5: Lage der Stationen Mongu, Maun, Malopeni und Skukuza innerhalb des Modellierungsgebietes.

Entsprechend dieser Beschreibungen wurde die Aufteilung in Primär- und Sekundärvegetation für die Modellierung der Gebiete um die Stationen gewählt. Für Skukuza liegt die längste Messperiode vor, die allerdings mehrere Lücken aufweist. Die Messungen erfolgten ab April 2000 und dauerten bis November 2008 an. Die Lücken umfassen Januar und Dezember 2001, Dezember 2002, Oktober 2003 bis Januar 2004, Dezember 2004 bis Februar 2005 sowie Januar bis September 2006. Dennoch können für diese Station 85 Monate verglichen werden. Für den Vergleich der Modellergebnisse mit den Stationsdaten wurde für die Pixel, die innerhalb eines Radius von einem Kilometer um die Station liegen, der Mittelwert der NPP bestimmt. Dies wird als das Gebiet angenommen, dessen Vegetation mit homogener Verteilung die Messungen beeinflusst, wenn diese in einer Höhe von etwa $15 \mathrm{~m}$ erfolgen (Rannik et al., 2006).

Die Ergebnisse aus den Vergleichen an den vier Stationen fallen sehr unterschiedlich aus, was auch auf die unterschiedlichen Messperioden zurückgeführt werden kann. Ein Vergleich des zeitlichen Verlaufes (Abbildung 4.6) zeigt, dass bis auf die Ergebnisse der Station Malopeni die Modellwerte etwa um einen Faktor zwei bis drei unter den Messwerten 
der EK-Türme liegen. Dies kann hauptsächlich auf die Unterschiede der betrachteten Randbedingungen zurückgeführt werden. Die äußeren Einflüsse der direkten Umgebung auf die Messwerte der NEE, wie Feuerereignisse oder die Beeinflussung der Messungen durch Tierherden führen größtenteils zu einer Erhöhung der Werte. Dadurch stehen niedrigen Modellergebnissen meist wesentlich höhere Messwerte gegenüber. In den Streudiagrammen (Abbildung 4.7 und Abbildung 4.8) ist dieser Effekt deutlich an der Häufung von geringen Modellwerten und einem entsprechend breitem Wertebereich der zugehörigen Stationswerte zu erkennen.

(a)

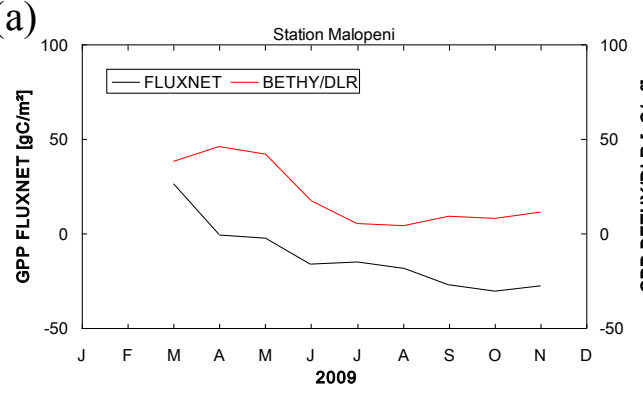

(c)

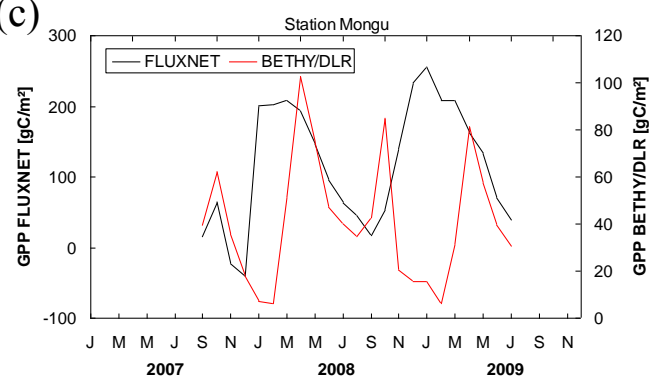

(b)

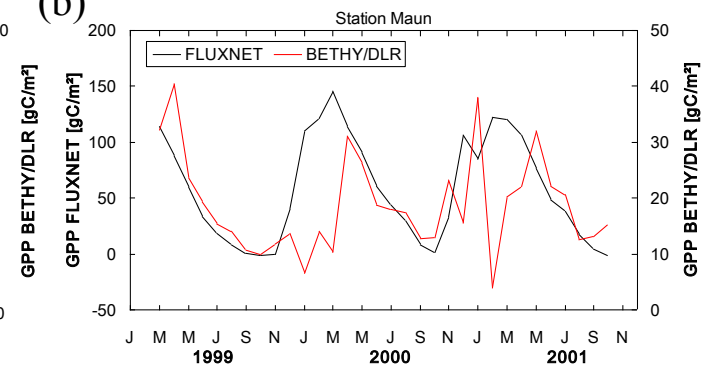

(d)

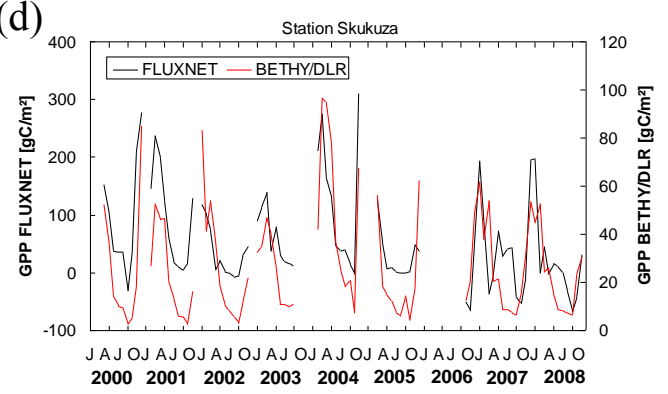

Abbildung 4.6: Vergleich der Modellergebnisse (rot) der GPP mit aus Eddy-Kovarianz-Messungen abgeleiteten Werten (schwarz) an vier Stationen im Untersuchungsgebiet. Links oben Malopeni, Krüger-Nationalpark, Südafrika; rechts oben Maun, Botswana; links unten Mongu in Simbabwe; rechts unten Skukuza, Krüger-Nationalpark, Südafrika.

Die kurze Zeitspanne der Malopeni-Messungen erschwert die Interpretation des Vergleiches an dieser Station. Zwar befinden sich die GPP-Werte innerhalb des gleichen Wertebereiches, eine Korrelation kann allerdings nicht ausgemacht werden. Dies gilt sowohl für Tageswerte (Abbildung 4.7) als auch für die Monatssummen (Abbildung 4.8). Eine bessere Ausgangslage bieten die ab knapp drei Jahre umfassenden Messreihen der Stationen Maun und Mongu. Dabei ist in Abbildung 4.6b, c und d auffällig, dass die Verläufe bei negativen Steigungen eine bessere Übereinstimmung haben als bei positiven Steigungen. Die Unterschätzung der GPP durch das Modell beträgt für die Station Maun etwa einen Faktor von drei, für die Station Mongu von ungefähr zwei (unterschiedliche Skalen in Abbildung 4.6). Für die Station Skukuza ergibt sich eine Unterschätzung von etwa 1.5. Die relativ lange Messreihe von knapp neun Jahren bietet eine gute Grundlage für den Vergleich. Allerdings 
treten in den Messdaten immer wieder Lücken auf, die meist nur einzelne Monate in der Phase der maximalen Aktivität der Vegetation (Dezember, Januar und Februar) betreffen. Außerdem fehlt nahezu das komplette Jahr 2006. Dennoch können die Verläufe über längere Zeiträume verglichen werden, wobei die Phasen der photosynthetischen Aktivität in beiden Datenreihen klar getrennt werden können. Das Ansteigen und Absinken der Kohlenstoffaufnahme der Berechnungen deckt sich in der Regel mit dem jeweiligen Verlauf der Messungen. Stellenweise gibt es aber auch Abweichungen bei kurzfristigen Variationen in beiden Datenreihen.

(a)

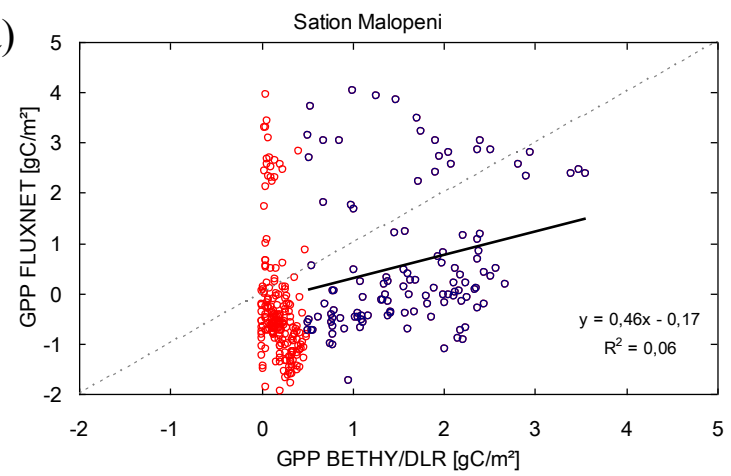

(c)

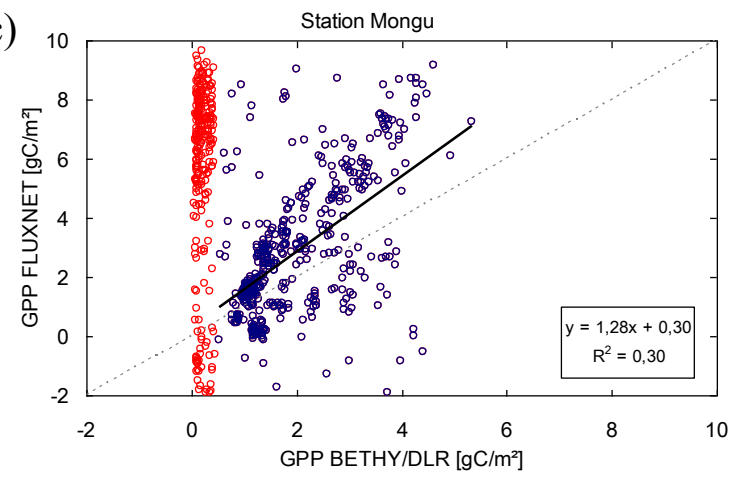

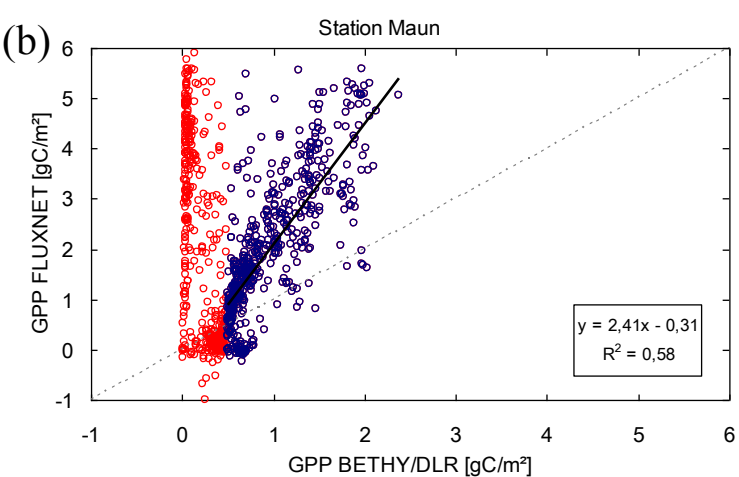

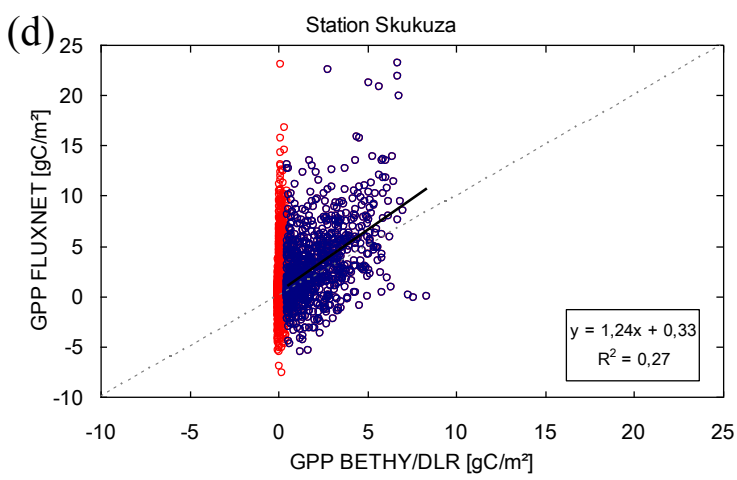

Abbildung 4.7: Streudiagramme von täglichen Modellergebnissen und Messwerten an den vier FLUXNET-Stationen. Werte unter $0.5 \mathrm{gC} \mathrm{m}^{-2} \mathrm{~d}^{-1}$ (rot) werden bei dem Vergleich nicht berücksichtigt. Die gestrichelte, graue Linie kennzeichnet die 1:1-Gerade.

Ein qualitativer Vergleich erfolgt über die Bestimmung der Korrelationen zwischen den Messwerten und den Modellergebnissen, sowohl der Tageswerte (Abbildung 4.7) als auch der Monatswerte (Abbildung 4.8). Schließlich wird die relative Abweichung der Modellwerte von den Turmmessungen an den vier Stationen bestimmt über:

$$
\varepsilon_{r}=\frac{\sum_{i=1}^{n}\left(\frac{G P P_{B E T H Y} / D L R, i}{G P P_{F L U X N E T, i}-G P P_{F L U X N E T, i}}\right)}{n} \cdot 100
$$


(a)

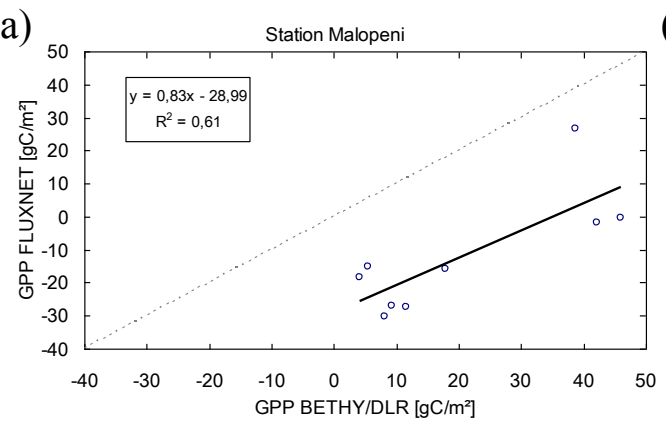

(b)

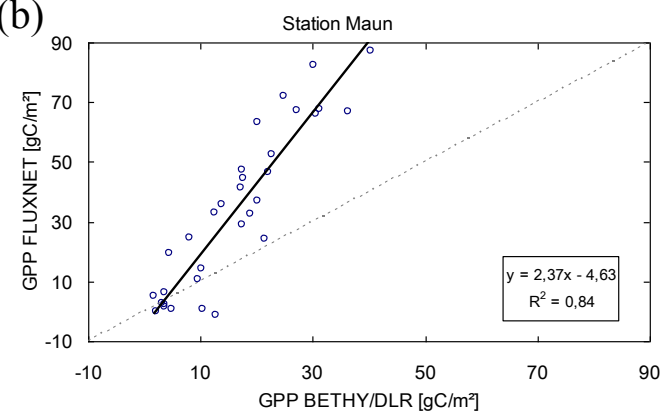

(c)

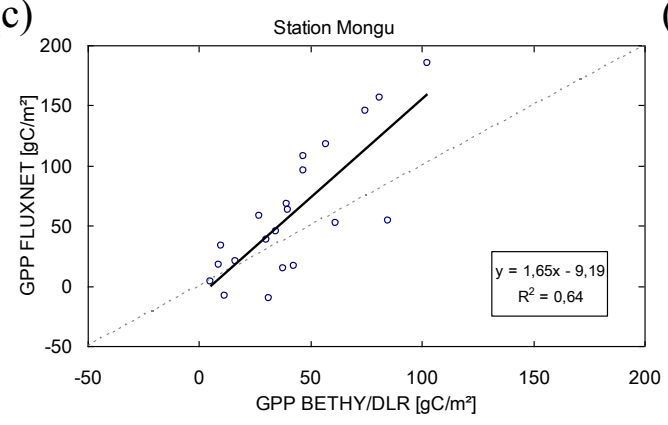

(d)

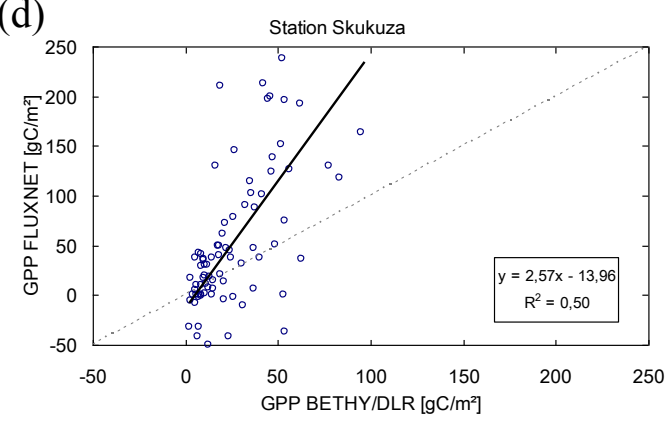

Abbildung 4.8: Streudiagramme der Monatssummen aus den Modellergebnissen und den Stationsmessungen. Die gestrichelte, graue Linie kennzeichnet die 1:1-Gerade.

Die bereits erwähnte Häufung von geringen Werten der GPP aus dem Modell bei einer breiten Verteilung der Messwerte wird auf die Unterschiede in der Betrachtung der Randbedingungen zurückgeführt. Sind diese Bedingungen im Modell ungünstig für die photosynthetische Aktivität der Vegetation, werden entsprechend geringe Raten der GPP berechnet. Es ist anzunehmen, dass in solchen Fällen bei der Messung der $\mathrm{CO}_{2}$-Flüsse äußere Faktoren weiterhin das Ergebnis beeinflussen. Dieser Unterschied in den Datensätzen kann teilweise reduziert werden, indem die niedrigen Werte der Modellergebnisse und die zugehörigen Messungen aus dem Vergleich heraus genommen werden. Als Grenzwert wird eine Produktivität von weniger als $0.5 \mathrm{gC} \mathrm{m}^{-2} \mathrm{~d}^{-1}$ festgelegt (Abbildung 4.7), womit die angesprochenen Häufungen bei allen Stationen erfasst werden.

Für die übrigen Werte ist für die Stationen Maun und Mongu ein Zusammenhang erkennbar, wobei die Produktivität durch das Modell mehrheitlich unterschätzt wird. Dabei ist die Streuung der Werte der Station Mongu wesentlich höher, was sich auf das berechnete Bestimmtheitsmaß auswirkt ( $\mathrm{R}^{2}=0.30 \mathrm{im}$ Gegensatz $\mathrm{zu} \mathrm{R}^{2}=0.58$ für Maun). Ähnliches gilt für die Station Skukuza, für die aufgrund der hohen Zahl an Messwerten und der breiten Streuung anhand des Diagramms kein linearer Zusammenhang erkennbar ist. Die GPP wird hier bei hohen Werten durch das Modell unterschätzt, bei niedrigen Werten häuft sich allerdings die Anzahl an überschätzten Modellwerten. 
Die Station Malopeni stellt in dem Vergleich einen Sonderfall dar, da hier als einzige Station eine Überschätzung der GPP durch das Modell erkennbar ist. Allerdings ergibt sich hier aufgrund der kurzen Messperiode und der hohen Streuung ein sehr niedriges Bestimmtheitsmaß von 0.06. Gleiches gilt ebenso für die relative Abweichung der Stationsdaten gegenüber den Modellergebnissen von 287.8\%. Die übrigen Stationen liegen mit Werten von $-53.2 \%$ für die Station Maun, $-36.0 \%$ für Mongu und $-17.6 \%$ für die Station Skukuza deutlich darunter.

Bei Betrachtung der Monatssummen (Abbildung 4.8) verbessert sich das Bestimmtheitsmaß für Malopeni zwar auf 0.60, ist aber aufgrund des kurzen Messzeitraumes (9 Monate) wenig repräsentativ. Für die restlichen Stationen ergibt sich auch für die monatlichen Werte eine systematische Unterschätzung der GPP durch das Modell. Das Bestimmtheitsmaß variiert stark zwischen 0.84 für die Station Maun, 0.64 für Mongu und 0.5 für Skukuza. Die Unterschätzung für Mongu und Maun durch das Modell ändert sich nicht wesentlich gegenüber der der Tageswerte, für Skukuza wird diese für den gesamten Wertebereich deutlicher. Im Gegensatz zum Bestimmtheitsmaß erhöht sich die relative Abweichung aller Stationen außer für Mongu (-33.0\%) gegenüber der Betrachtung der Tageswerte. Malopeni erreicht dabei wiederum den größten Wert von $+136.5 \%$. Der Wert für Maun (-50.8\%) ändert sich aufgrund der geringeren Streuung sehr wenig. Dagegen erhöht sich die Abweichung für Skukuza auf $-40.6 \%$. Für die weitere Betrachtung ist festzuhalten, dass bis auf den unverhältnismäßig hohen Fehler der Station Malopeni alle Abweichungen eine Unterschätzung der Modellergebnisse gegenüber den Messwerten darstellen.

Krinner et al. (2005) verglichen Modellwerte des Dynamic Global Vegetation Model (DGVM) ORCHIDEE mit FLUXNET-Messungen der NEE für verschiedene Vegetationstypen. Dabei wurden für Grasland deutlich schlechtere Übereinstimmungen als für bewaldete Gebiete festgestellt. Insgesamt stellt der spärliche Bewuchs der Savanne einen großen Unsicherheitsfaktor in der Berechnung der Ökosystemrespiration $R_{\text {eco }}$ für die Bestimmung der GPP dar. Je größer der Anteil an blankem Boden im Messgebiet ausfällt, umso höher wird der Beitrag der heterotrophen Respiration, der ebenfalls nicht im Modell bestimmt wird. Wird dieser Beitrag fehlerhaft berechnet, geht dieser direkt auf die berechnete GPP über.

Sowohl der systematische Fehler als auch die zufällige Abweichung der Eddy-KovarianzMessungen werden mit etwa 30\% für die halbstündigen Messungen angenommen (Businger, 1986; Moncrieff et al., 1996, Law et al., 2001). Zufällige Abweichungen treten vor allem durch die Messung des turbulenten Flusses an nur einem Punkt auf. Hinzu kommt die Variation der Bodenbedeckung, unzureichende Länge der Messreihe und zufälliges 
Rauschen als Teil des Messsignals. Als systematische Fehler können unter anderem die fehlerhafte Kalibrierung der Sensoren, unzureichende Höhe des Sensors über der Vegetation oder die Verwendung einer fehlerhaften Transferfunktion ausgemacht werden. Für die Berechnung der Tages- und Monatssummen wird angenommen, dass sich die zufällige Abweichung herausmittelt und gegenüber dem systematischen Fehler vernachlässigbar ist.

Eine weitere bekannte Fehlerquelle der GPP aus den FLUXNET-Daten ist die Berechnung der Ökosystemrespiration $R_{\text {eco }}$. Ein Vergleich verschiedener Modelle zur Bestimmung von $R_{\text {eco }}$ durch Richardson et al. (2006) hat gezeigt, dass Unterschiede von bis zu 40\% zwischen den Ergebnissen auftreten können, je nachdem welches Modell für $R_{\text {eco }}$ verwendet wurde. Die Modelle unterscheiden sich in der Bestimmung der Temperaturabhängigkeit der Respiration, durch die mittels Regression aus den Nachtmessungen die Tageswerte berechnet werden. Daher ist für die Berechnung der Ökosystemrespiration von einem Fehler von 30-40\% auszugehen. Als wesentlicher Grund für den hohen Fehler des Modells wird die Verwendung von nur einem freien Parameter $E_{0}$ aufgeführt. Ein besseres Ergebnis wird erzielt, wenn $T_{0}$ und $T_{\text {ref }}$ ebenfalls als freie Parameter in die Berechnung eingehen (Falge et al., 2001). 


\subsection{Modellvergleiche}

Eine indirekte Möglichkeit die Qualität der Modellergebnisse zu bewerten besteht darin, diese mit den Ergebnissen anderer, validierter Modelle zu vergleichen. Allerdings sind solche Datensätze speziell für das betrachtete Arbeitsgebiet nicht beliebig verfügbar. Dazu muss auf globale Produkte zurückgegriffen werden, die für unterschiedliche Zeiträume zugänglich sind. Zur Anwendung kommt hier ein NPP-Produkt, abgeleitet aus AVHRRDaten und GPP- und NPP-Produkte berechnet aus MODIS-Daten. Ersteres wurde am Geographischen Institut der Universität Maryland für 1981 bis 2000 mit Hilfe des Global Production Efficiency Model (GloPEM) erstellt und umfasst die NPP in 8km Auflösung (Prince und Small, 2003; Prince und Goward, 1995). Die MODIS-Produkte sind für den Zeitraum 2000 bis 2010 durch die Numerical Terradynamic Simulation Group (NTSG) der Universität von Montana prozessiert worden (Zhao et al., 2005, 2006).

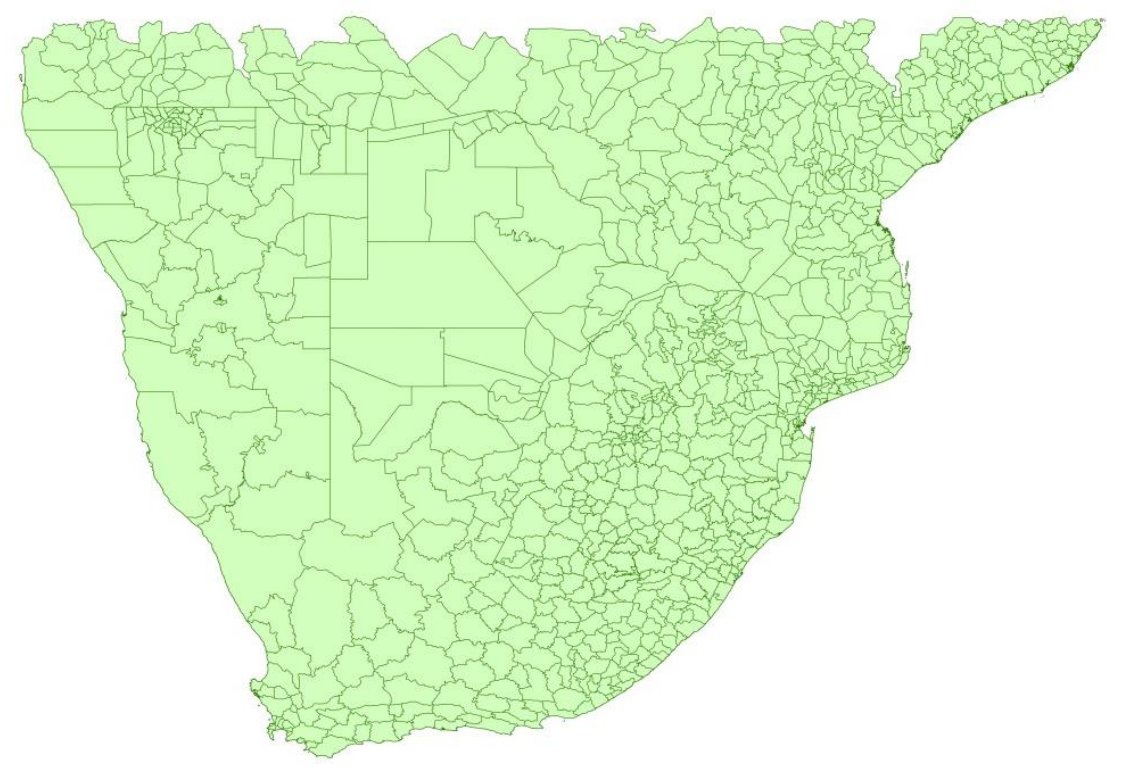

Abbildung 4.9: Verteilung der administrativen Distrikte der Nationen im südlichen Afrika, zur Bestimmung der NPP- und GPP-Summen für den Modellvergleich.

Zur Reduzierung der Datenmenge und der Fehler aufgrund unterschiedlicher Projektionen werden die Summen für die jeweils höchsten Stufen der administrativen Ebenen der einzelnen Länder berechnet. Dies verhindert außerdem eine breite Streuung bei der Gegenüberstellung der Datensätze. Die Stufen gehen im Detail für Angola und Mozambik über drei Ebenen, für Botsuana, Namibia, Südafrika, Sambia und Simbabwe über zwei und für Lesotho und Swasiland nur über eine. Die Verteilung der betrachteten Distrikte ist in Abbildung 4.9 gezeigt. Gebiete, die nicht vollständig innerhalb des Arbeitsgebietes liegen (also teilweise nördlich von $15^{\circ}$ Süd), wurden nicht berücksichtigt. 


\subsubsection{Das AVHRR-NPP-Produkt}

Das Modell GloPEM (Prince und Goward, 1995) beruht auf der Methode, aus der absorbierten photosynthetisch aktiven Strahlung (APAR) die Produktivität der Vegetation zu bestimmen. Dazu wird eine Verwertung bzw. Umwandlungsrate der APAR in gespeicherten Kohlenstoff in Form der GPP angenommen. Nach Abzug der Erhaltungsatmung ergibt sich entsprechend die NPP. Für die anfängliche Berechnung der PAR wird ebenfalls der Ansatz nach Stull (1988) (siehe Kapitel 2.4.1) mit Informationen über die Bewölkung aus Beobachtungen des Total Ozone Mapping Spectrometer (TOMS) verwendet. Ein spektraler Vegetationsindex wird aus den Wellenlängen im Sichtbaren und Infraroten der AVHRRReflexionen gewonnen und mit der einfallenden PAR zur absorbierten Strahlung (APAR) kombiniert (Prince und Goward, 1995). Dies geschieht in Zeitschritten von zehn Tagen. Die photosynthetische Aktivität wird schließlich unter Berücksichtigung von Stressfaktoren, abhängig von Bodenfeuchte und Lufttemperatur, bestimmt. Ein Vergleich der GloPEM-NPP mit lokalen Messwerten der $\mathrm{CO}_{2}$-Aufnahme der borealen Wälder im Norden Kanadas zeigte eine breite Variation mit Unterschätzungen von $8 \%$ im Süden und Überschätzungen von bis zu 60\% im Norden des BOREAS-Gebietes (Goetz, 1999). Beide Werte gelten für immergrünen Nadelwald.
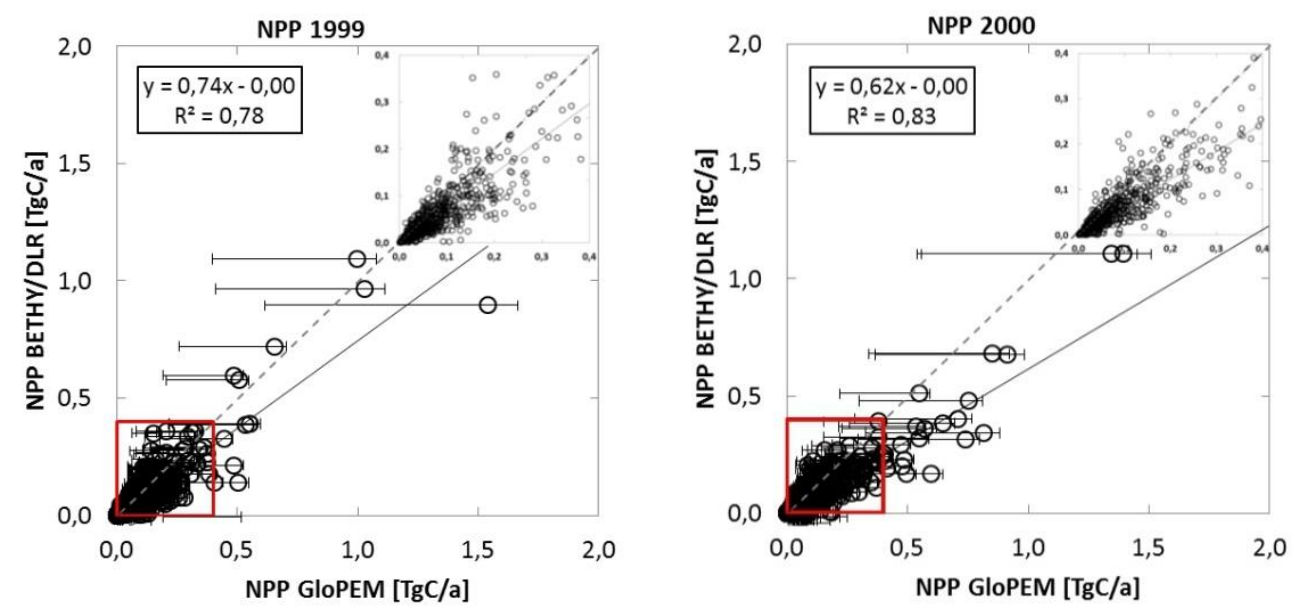

Abbildung 4.10: Vergleich der NPP-Ergebnisse aus den Modellen BETHY/DLR und GloPEM für 1999 (links) und 2000 (rechts). Innerhalb der Diagramme ist jeweils der Bereich niedriger Werte dargestellt.

Die zeitliche Überschneidung des GloPEM-Datensatzes und der NPP-Daten aus BETHY/DLR beschränkt sich auf die Jahre 1999 und 2000. Der Vergleich in Abbildung 4.10 zeigt eine gute Korrelation zwischen den beiden Datensätzen mit einem Bestimmtheitsmaß von 0.78 für 1999 und 0.83 für 2000. Die Werte aus den GloPEM-Daten werden durch die Ergebnisse von BETHY/DLR signifikant unterschätzt um einen Faktor 
von etwa 1.4 für 1999 und 1.6 für 2000. Allerdings wurde bereits in Goetz et al. (1999) von der Tendenz der Überschätzung durch GloPEM berichtet, wenngleich diese Studie in borealen Gebieten durchgeführt wurde. Dies kommt dennoch dem gezeigten Bild von erhöhten Werten gegenüber denen des BETHY/DLR-Modells entgegen. Die Ergebnisse von BETHY/DLR liegen darüber hinaus innerhalb des Fehlerbereiches des Vergleichsmodells, wobei sich eine mittlere Abweichung von 8\% für 1999 und 15\% für 2000 ergibt.

\subsubsection{MODIS-GPP- und NPP-Produkte}

Das MODIS-GPP-Produkt MOD17 wird nach dem Ansatz von Monteith $(1972,1977)$ bestimmt, wonach die Photosyntheseleistung mit der absorbierten photosynthetisch aktiven Strahlung (fPAR) korreliert:

$$
G P P=\varepsilon \cdot f P A R \cdot P A R
$$

Dabei ist $\varepsilon$ die Lichtnutzungseffizienz der Vegetation (engl. Light Use Efficiency, LUE). Diese bestimmt sich aus der maximalen LUE $\varepsilon_{\max }$, die vom Vegetationstypus, einem Limitierungsfaktor für tiefe Temperaturen $m\left(T_{\min }\right)$ und einem für ein erhöhtes Dampfdruckdefizit (engl.: Vapor Pressure Deficit, VPD $m(V P D)$ abhängig ist (Heinsch et al., 2003):

$$
\varepsilon=\varepsilon_{\max } \cdot m\left(T_{\min }\right) \cdot m(V P D)
$$

Die Limitierungsfaktoren $m\left(T_{\min }\right)$ und $m(V P D)$ werden zusammen mit der maximalen $\varepsilon_{\max }$ für das entsprechende Biom einer Tabelle entnommen (Heinsch et al., 2003). Informationen über die Verteilung der Biome folgen aus dem MODIS-Land-Cover-Produkt MOD12Q1, die fPAR wird aus dem MOD15-LAI/fPAR-Produkt bezogen.

Die NTSG-MOD17-Produkte GPP und NPP werden mit bereinigtem MODIS-FPAR/LAI Eingangsdatensatz berechnet. Die Korrektur von wolkenkontaminierten fPAR-Datensätzen führt zu einer geringeren Unterschätzung der GPP (Zhao et al., 2005). Eine Evaluierung der MODIS-GPP- und NPP-Produkte auf globaler Ebene (Zhao et al., 2005) zeigte eine gute Übereinstimmung von globalen Gesamtsummen. Dabei ist allerdings davon auszugehen, dass sich bei einer globalen Betrachtung Abweichungen in einzelnen Regionen herausmitteln. Dafür spricht auch die Validierungsstudie von Turner et al. (2006), die für mehrere Stationen der BigFoot-Messkampagne für NPP und GPP gleich viele Unter- wie Überschätzungen fanden, je nach Vegetationstyp. Der Vergleich mit 15 FLUXNETStationen (Heinsch et al., 2006) zeigte ebenfalls eine hohe Korrelation der GPP ( $\mathrm{r}=0.859 \pm$ 0.173), darüber hinaus eine Überschätzung der GPP an den meisten Türmen, mit einer mittleren Abweichung von $+24 \%$. Für ein Savannengebiet wurde ein Fehler von $+30 \%$ 
bestimmt, für offenes Buschland auch bis zu 108\%. Turner (2005) berichtet eine Überschätzung der MODIS-GPP und daraus folgend der NPP um einen Faktor fünf gegenüber Messungen im Rahmen des BigFoot Programms, wogegen die berechnete autotrophe Atmung gute Übereinstimmung lieferte.
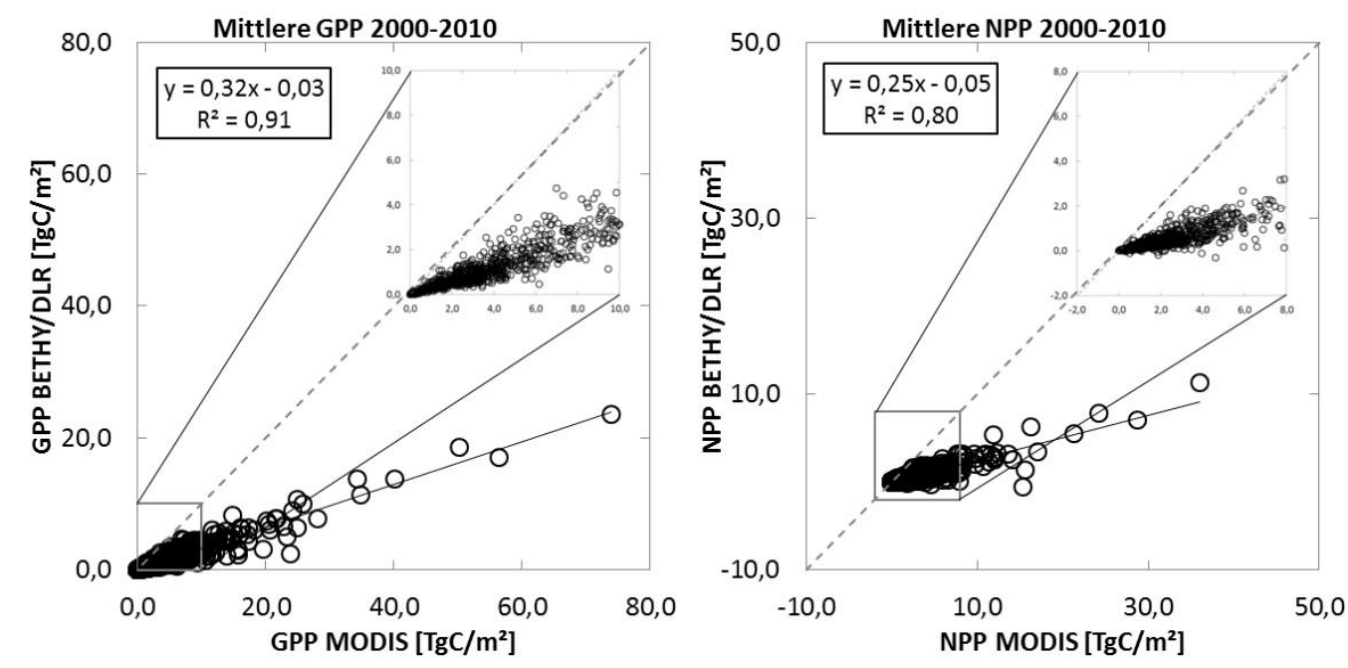

\begin{abstract}
Abbildung 4.11: Vergleich der Modellergebnisse aus BETHY/DLR und MODIS für die GPP (links) und die NPP (rechts). Die gestrichelte Linie stellt die 1:1-Linie dar. Innerhalb der Abbildungen ist der Bereich kleinerer Werte vergrößert dargestellt.
\end{abstract}

Der Vergleich der Modellergebnisse aus BETHY/DLR mit den MODIS-Produkten (Abbildung 4.11) ergibt für GPP und NPP zunächst ein hohes Bestimmtheitsmaß (0.91 und 0.80 entsprechend). Die angesprochene Überschätzung der MODIS-Werte gegenüber Bodenmessungen spiegelt sich auch hier wieder. Sowohl GPP als auch NPP aus BETHY/DLR liegen deutlich unter den Werten der MODIS-Produkte. Für die GPP ergibt sich im Mittel ein Faktor von 3.1, für die NPP sogar von 4.3, was relativen Abweichungen von $69 \%$ und $77 \%$ entspricht. In Anhang I sind ergänzend die Streudiagramme aus den Vergleichen der einzelnen Jahre aufgeführt.

Zum Abschluss der Fehlerbetrachtung fällt es schwer einen endgültigen Fehler für die berechneten Größen der GPP und der NPP festzulegen. Insgesamt bleibt festzustellen, dass es schwierig ist, geeignete Vergleichsmessungen oder -produkte zu erhalten, über die die Güte der Modellergebnisse bestimmt werden kann. Im Falle der Biomassemessungen im Krüger-Nationalpark fehlen generell weitere Arbeiten, die eine Aussage über die Qualität der Messungen selbst zulassen. Dennoch stellen die Messungen den geeignetsten Datensatz unter den verfügbaren dar, um ihn mit den berechneten Zuwachsraten des Modells BETHY/DLR zu vergleichen. Allerdings sollten die Ergebnisse streng genommen nur für die berechneten Assimilationsraten von Grasland mit C4-Mechanismus verwendet werden, 
da andere Vegetationstypen in diesem Vergleich nicht berücksichtigt wurden. Starke Variationen werden nicht nur dem Modell alleine zugeschrieben, sondern auch der Unsicherheit die der Messmethode zugrunde liegt. Es ergibt sich schließlich eine Überschätzung der NPP-Werte durch das Modell für Grasland, mit einem relativen Fehler von 50\%.

Der Vergleich der Messungen an den FLUXNET-Stationen zeigt zusätzlich, dass für die GPP von sommergrünem Laubwald ein Fehler von 40-50\% realistisch ist, mit Korrelationen zwischen 0.5 und 0.85. Allerdings konnte hier eine Unterschätzung der Produktivität festgestellt werden. Auch ist zu bedenken, dass die FLUXNET-Daten der GPP ebenfalls nur Ergebnisse einer Modellrechnung sind, denen nicht unerhebliche systematische Fehler unterliegen und somit auch keine direkten Bodenmessungen für den Vergleich vorliegen.

Gleiches gilt für den Vergleich der BETHY/DLR-Ergebnisse mit Produkten wie GloPEModer MODIS-GPP bzw. -NPP. Aus den Vergleichen mit den beiden Produkten ergeben sich sehr gute Korrelationen von mindestens 0.78 bis 0.91 , wobei zu beiden Produkten eine signifikante Unterschätzung festgestellt werden kann (Faktor 1.4 bis 1.6 für GloPEM und 3.1 bis 4.3 für MODIS). Die relative Abweichung von den GloPEM-Werten fällt mit maximal 15\% sehr gering aus im Vergleich zur Abweichung von den MODIS-Werten bis zu 77\%. Die Unsicherheit dieser Vergleiche mit anderen Modellergebnissen wurde bereits diskutiert. Auffällig ist dennoch, dass in anderen Arbeiten sowohl für das GloPEM-Produkt als auch für das MODIS-Produkt eine starke Überschätzung der GPP bzw. der NPP bestimmt wurde. Weitere Untersuchungen mit weiterführenden Messungen des Biomassezuwachses sind unbedingt notwendig und auch im Rahmen künftiger Arbeiten und Projekte geplant. 


\section{$5 \quad$ Der Degradationsindex}

Wie einführend zu dieser Arbeit dargestellt, fehlt es noch immer an einer objektiven, systematischen und räumlich genauen Methode zur Messung von Landdegradation über längere Zeiträume hinweg. Daher sollen die vorgestellten Ergebnisse des SVAT-Modells BETHY/DLR verwendet werden, um großräumig für das Arbeitsgebiet des südlichen Afrika Zeitreihen der Pflanzenproduktivität zu analysieren. Daraus können Rückschlüsse auf deren natürliche und durch den Menschen beeinflusste Veränderungen gezogen werden. Dazu werden akkumulierte Summen der NPP über die Vegetationsperioden verwendet und deren Entwicklung über den gesamten Modellierungszeitraum betrachtet. Berechnet wird daraus der Gesamttrend, der sich aus dieser Entwicklung für jedes einzelne Pixel ergibt. Dem gegenübergestellt wird zunächst die Landnutzung durch den Menschen und schließlich die Entwicklung der klimatischen Bedingungen während des betrachteten Zeitraumes. Daraus kann letztlich bewertet werden, in wie weit positive oder negative Trends der vegetativen Produktivität auf Einflüsse des Menschen oder der klimatischen Gegebenheiten zurückgeführt werden können.

\subsection{Trends in der Vegetationsproduktivität}

Für die Betrachtung der Entwicklung der Vegetation über den gesamten Modellierungszeitraum von zwölf Jahren, werden zunächst Summen über die zugehörigen elf Vegetationsperioden (99/00 bis 09/10) gebildet. Diese werden von Juli (ab dem 182. Tag des Jahres) bis Juni des Folgejahres (bis Tag 181) bestimmt. In Abbildung 5.1 ist die Zeitreihe der täglichen NPP-Werte eines einzelnen Pixels zu sehen (blaue Linie), wie sie vom Modell berechnet werden. Die Tageswerte werden dann über die Periode eines Jahres aufsummiert (rote Kreise). Das zugehörige Pixel entspricht einem mit Grasland bewachsenem Gebiet in der südafrikanischen Provinz Nordkap nahe der Grenze zu Botswana (26.67S 21.93E). Anhand dieses Beispiels lässt sich der in dieser Arbeit verfolgte Ansatz erkennen. Der Verlauf der jährlichen Produktivität der dem Pixel zugrunde liegenden Vegetation zeigt einen deutlichen negativen Trend. Die Steigung des linearen Trends repräsentiert demnach den mittleren jährlichen Verlust an vegetativer Produktivität und ergibt sich in diesem Falle zu $1.39 \mathrm{gCm}^{-2} \mathrm{a}^{-1}$ bei einem Bestimmtheitsmaß von 0.67. Bei diesem mittleren jährlichen Verlust hat die Vegetation im betrachteten Pixel innerhalb der elf Jahre über die Hälfte (55\%) ihrer ursprünglichen Produktivität (27.67 $\mathrm{gCm}^{-2} \mathrm{a}^{-1}$ für 99/00) eingebüßt. Dieser relative Trend der NPP bezüglich der Anfangswerte der Periode 99/00 ist in Abbildung 5.2a für das gesamte Untersuchungsgebiet gezeigt. Blau stellt einen positiven Trend der Produktivität der Vegetation dar, rot einen negativen Trend. In der folgenden Diskussion werden nur die negativen Trends betrachtet. Abbildung 5.2b zeigt den zugehörigen Korrelationskoeffizen- 
ten des linearen Fits. Innerhalb des Arbeitsgebietes werden einzelne Bereiche deutlich, die sich durch einen besonders hohen negativen Trend hervorheben. Diese lassen sich auf spezielle Bodentypen zurückführen, die innerhalb der Formulierung des Bodenmodells schlechte Wasserspeicher darstellen oder schlicht nicht tief genug sind, um ausreichend Wasser zur Versorgung der Vegetation aufnehmen zu können.

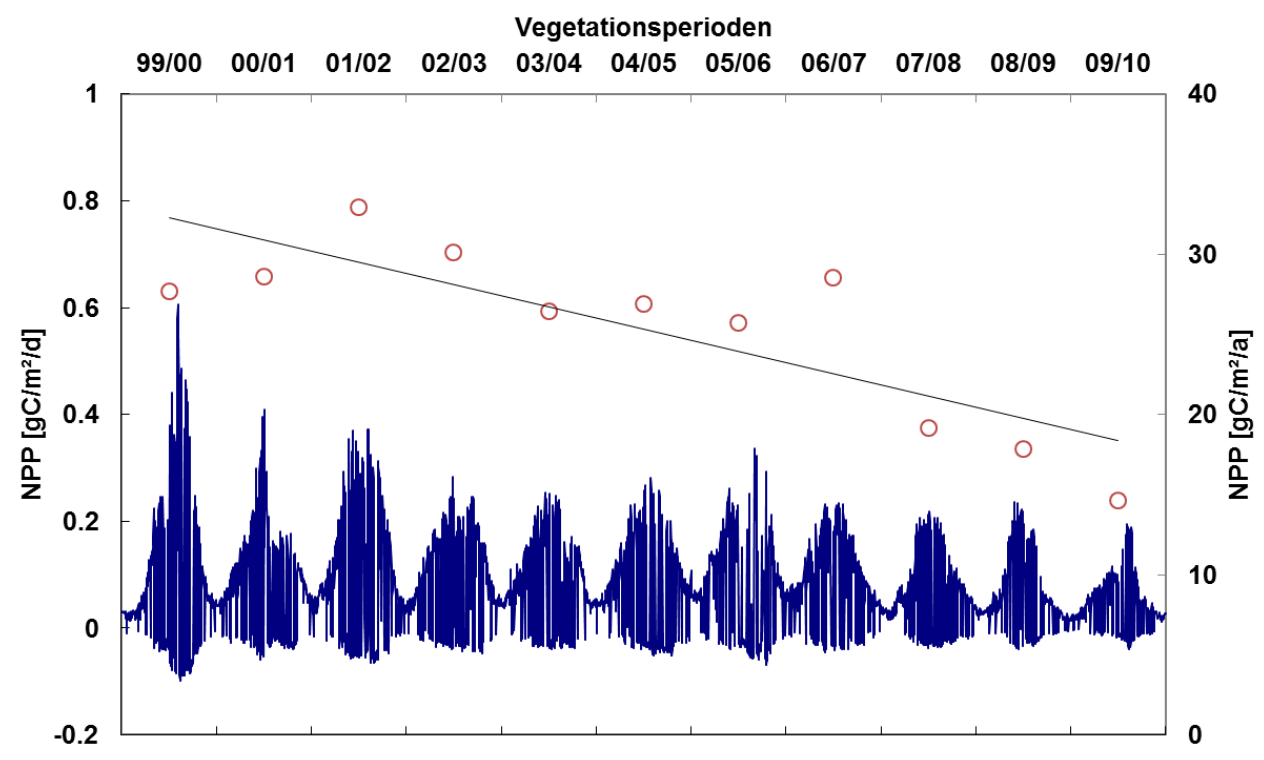

Abbildung 5.1: Zeitreihe der NPP als Tageswerte (blaue Linie) und Jahressummen (rote Kreise) über die Vegetationsperioden von 1999 bis 2010.

Die auffällige Form in Lesotho beispielsweise repräsentiert das Vorkommen des Bodentyps Lithosol mit einer Mächtigkeit von $10 \mathrm{~cm}$. Dieser Bodentyp ist meist in Bergregionen als dünne Bodenschicht auf dem Felsgestein vorzufinden. Im Gegensatz dazu besitzen die meisten Bodentypen Mächtigkeiten von über $120 \mathrm{~cm}$. Bei Bodentypen mit geringen Tiefen macht sich schnell der verwendete Mechanismus zur Berücksichtigung des Trockenstress bemerkbar. Aus den entsprechenden Bodenparametern kann das maximal für die Vegetation verfügbare Bodenwasser zu $2.4 \mathrm{~cm}$ Wassersäule bestimmt werden. Trockenstress wird dann simuliert, wenn im Boden nur noch $25 \%$ dieser maximalen Menge verfügbar ist, da dann die Saugspannung der Pflanzen nicht mehr ausreicht, um dem Boden weiteres Wasser $\mathrm{zu}$ entziehen. Wird in diesem Beispiel also eine Wassermenge von $0.6 \mathrm{~cm}$ unterschritten, gerät die Pflanze in Trockenstress. Bei Betrachtung der Niederschlagswerte dieses Pixels zeigt sich eine starke Variabilität über den betrachteten Zeitraum, so dass die Niederschlagssumme in den Perioden $99 / 00$ bis 02/03 und der Periode 05/06 bis zu 40\% über dem Durchschnitt liegt, in den restlichen Jahren dagegen deutlich (bis 30\%) darunter. Für die Perioden mit unterdurchschnittlicher Regenmenge gerät die Vegetation regelmäßig, teilweise auch ganzjährig, unter Trockenstress, so dass effektiv keine Assimilation von 
Kohlenstoff möglich ist. Andere Böden, wie beispielsweise der Luvisol, erweisen sich durch ihren sehr hohen Sandanteil (>70\%) als schlechte Wasserspeicher, wodurch das aufgenommene Wasser relativ schnell versickert und für die Vegetation verloren geht. Relevant ist dabei ebenfalls die Dichte des vegetativen Bewuchses, durch die die Menge des dem Boden entzogenen Wassers mit bestimmt wird. So wird bei der Übersetzung der GLC2000-Klassen 2 und 3 (geschlossener und offener sommergrüner Laubwald) der Bedeckungsgrad von 0.9 auf 0.65 reduziert (siehe Tabelle 2.8). Dies kann dazu führen, dass direkt benachbarte Pixel gegenläufigen Trends unterliegen, wenn auf dem gleichen Boden mit identischen klimatischen Bedingungen der Bedeckungsgrad derart variiert, dass das Bodenwasser für das dichter bewachsene Pixel über längere Zeiträume nicht mehr ausreicht, für das weniger dicht bewachsene Pixel allerdings noch genügend Wasser verbleibt.

(a)

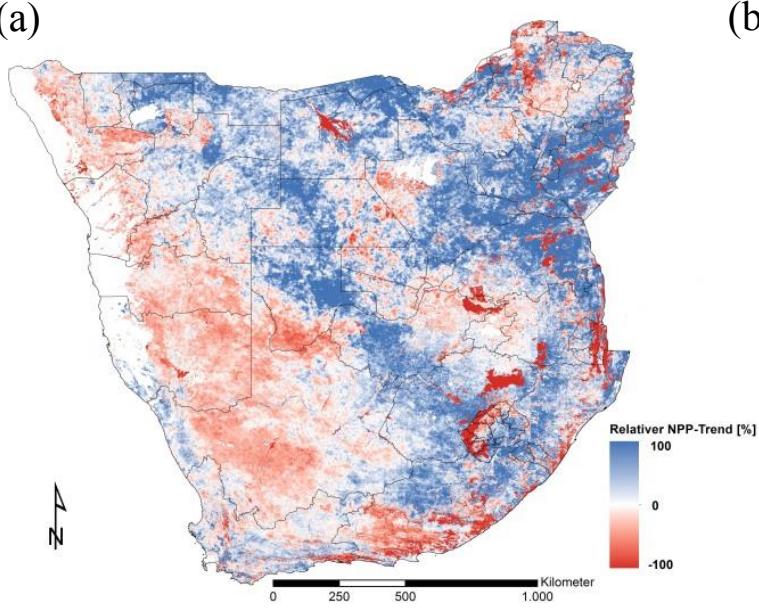

(b)

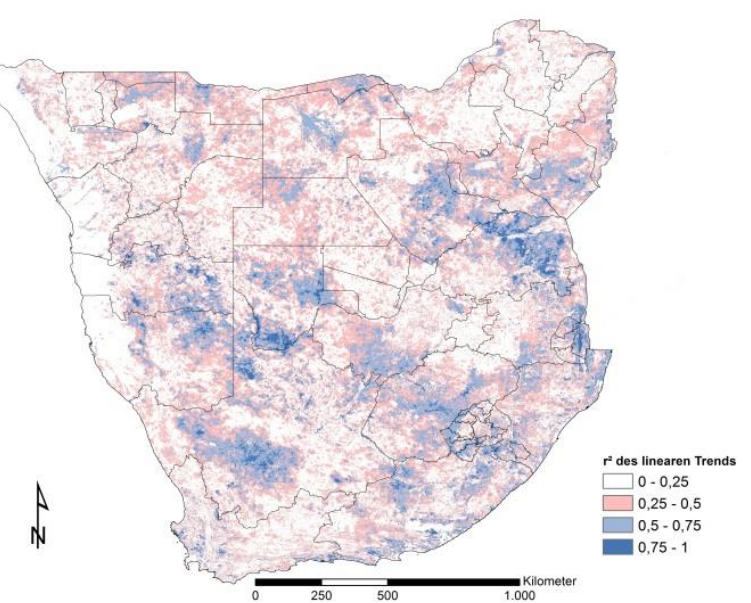

Abbildung 5.2: Karte der berechneten NPP-Trends für das gesamte Arbeitsgebiet aus den NPPSummen der einzelnen Vegetationsperioden (a) und der zugehörigen Korrelation des linearen Trends (b).

Die größten Flächen negativer Entwicklung der Produktivität sind im Westen in den trockenen, als Gras- und Buschland klassifizierten Gebieten Namibias und Südafrikas, zu finden. Außerdem ergibt sich für die landwirtschaftlichen Flächen um Pretoria und Johannesburg großflächig ein negativer Trend. Höhere Verluste vegetativer Produktivität ergeben sich in der Region um Port Elizabeth für Waldregionen von stellenweise bis zu 100\%. Bei Verlusten von $100 \%$ und mehr vollzieht sich ein Wechsel von einer Kohlenstoffsenke zu einer Quelle. Die berechnete NPP wird negativ, das bedeutet, dass die Pflanze durch die Atmung über das Jahr mehr Kohlenstoff an die Atmosphäre abgegeben hat, als sie aufnehmen konnte. Die angesprochenen Strukturen in Lesotho, in der südafrikanischen Provinz Neustaat, in Swasiland oder nördlich von Johannesburg zeigen den stärksten Rückgang der vegetativen Produktivität mit Raten von mehr als 100\%. Der ebenso hohe negative Trend für das Okavango-Delta wird im Gegensatz zu den vorherigen Regionen nicht alleine auf 
den Bodentyp zurückgeführt. Die Region zeichnet außerdem besonders die Klassifizierung als regelmäßig geflutetes Sumpfgebiet aus. Dieser Vegetationstyp besitzt eine sehr geringe Elektronentransportrate $J_{m}$, was die Photosyntheseaktivität sehr anfällig für Variationen in den klimatischen Bedingungen macht.

Der vorrangige Vegetationstyp im gesamten Arbeitsgebiet ist nach Tabelle 2.8 Buschland, gefolgt von Grasland. Aus der Verteilung der negativen Produktivitätstrends folgt dagegen eine höhere Anfälligkeit für Degradation der Graslandvegetation. Dies wird auf die intensive Weidelandnutzung im südlichen Afrika zurückgeführt. Durch die Abgrasung weiter Gebiete nehmen diese Ökosysteme erheblichen Schaden, der sich in der verringerten Produktivität der Pflanzen wiederspiegelt. Allerdings kann nicht der gesamte Rückgang der Produktivität auf die Beanspruchung durch den Menschen zurückgeführt werden. Auswirkungen haben dagegen vor allem auch Variationen der Niederschlagsmengen. Es muss außerdem geprüft werden, ob Veränderungen in der mittleren Jahrestemperatur zu drastischen Auswirkungen auf die Pflanzenwelt führen.

\subsection{Anomalien in klimatischen Zeitreihen}

Um feststellen zu können, ob ein negativer Trend in der Produktivität alleine der Bewirtschaftung des Naturraumes zuzuschreiben ist, muss ausgeschlossen werden, dass nicht zusätzlich widrige klimatische Bedingungen das Pflanzenwachstum beeinflusst haben. Daher werden für die im Modell verwendeten Zeitreihen des Niederschlags und der Temperatur analog wie in Kapitel 5.1 für die NPP ebenfalls Trends über die gesamte Modellierungsperiode erstellt (Abbildung 5.3).
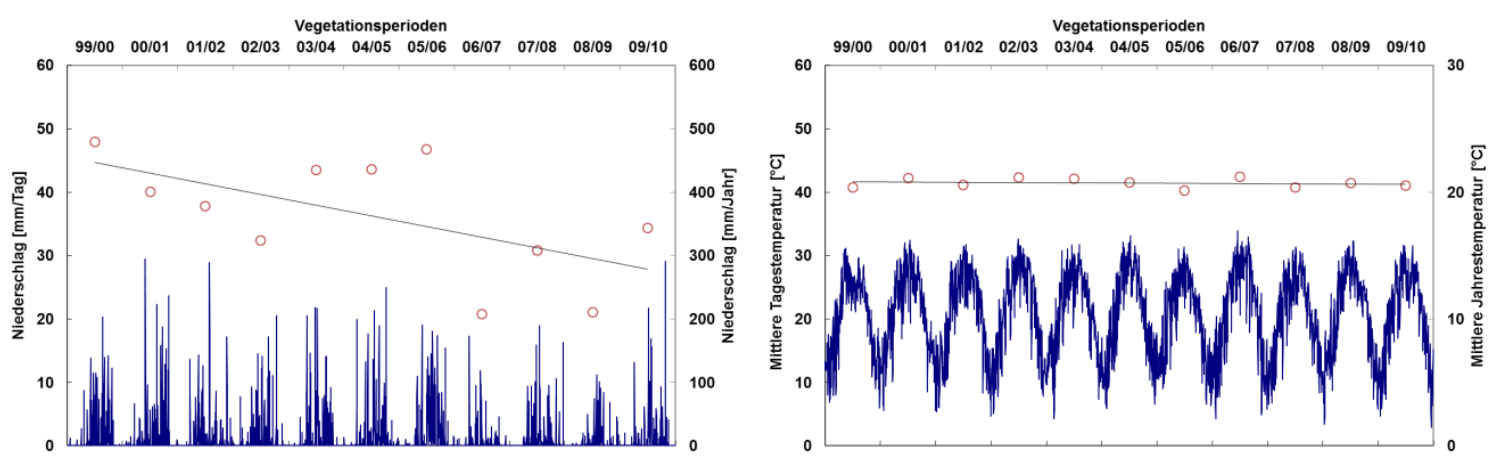

Abbildung 5.3: Zeitreihen des Niederschlags (a) und der mittleren Temperatur (b) über die Vegetationsperioden von 1999 bis 2010. Blaue Linien zeigen die Tageswerte, rote Kreise den Jahresniederschlag bzw. die mittlere Jahrestemperatur mit dem Trend über den gesamten Zeitraum (schwarze Linie).

Für das in Kapitel 5.1 beschriebene Pixel ergibt sich ein negativer Trend von $-16.8 \mathrm{~mm} \mathrm{a}^{-1}$ für den Niederschlag, allerdings mit einem $\mathrm{R}^{2}$ von 0.35 . Für die mittlere Temperatur ergibt 
sich ein schwacher negativer Trend von $-0.019{ }^{\circ} \mathrm{C} \mathrm{a}^{-1}$ mit einem $\mathrm{R}^{2}$ von 0.03 . Damit kann auch für andere Vegetationstypen unterschieden werden, ob sich die Vegetation trotz guter klimatischer Bedingungen zurückentwickelt hat oder weil die Bedingungen nicht optimal für das Wachstum waren.

(a)

(b)
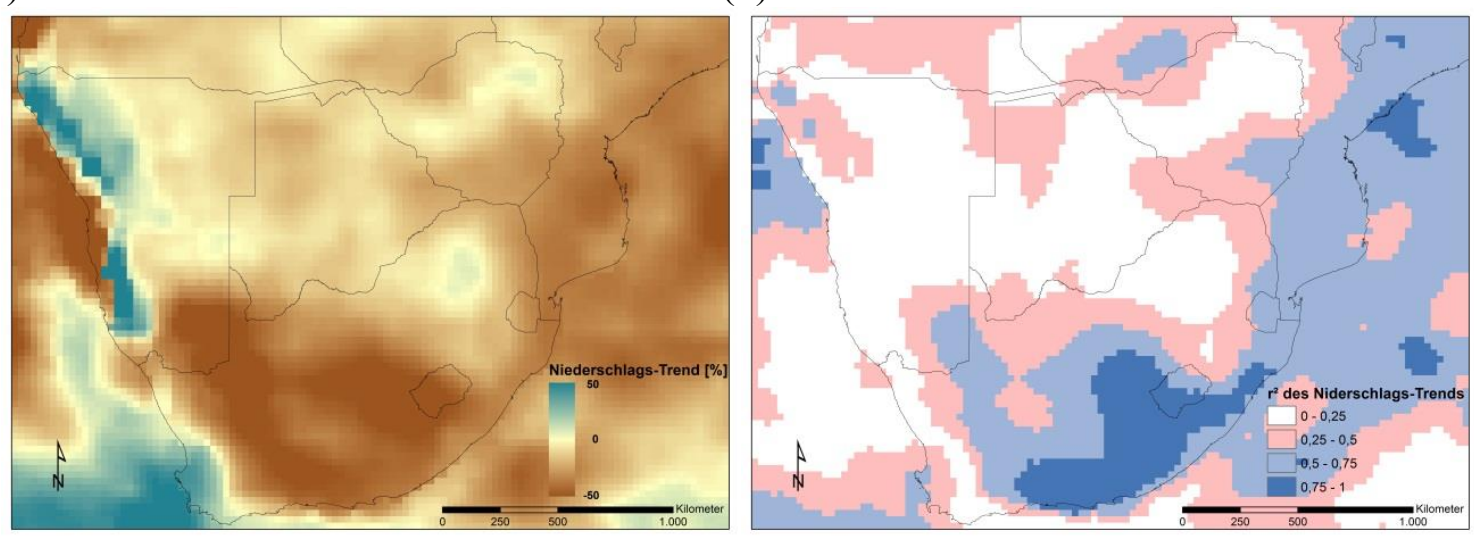

Abbildung 5.4: Relativer Trend des mittleren Jahresniederschlags (a) bezüglich des Wertes zu Beginn des Betrachtungszeitraumes 99/01-09/10 mit der zugehörigen Korrelation des linearen Fits (b).

(a)

(b)
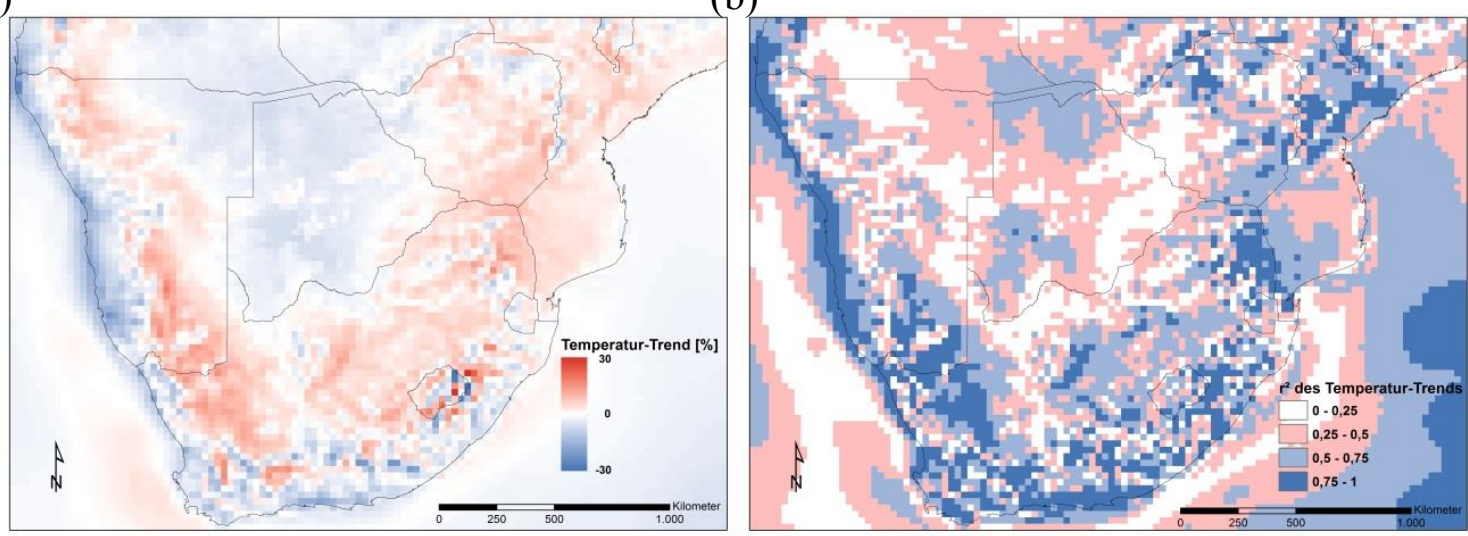

Abbildung 5.5: Relativer Trend der mittleren Temperatur (a) bezüglich des Wertes zu Beginn des Betrachtungszeitraumes 99/01-09/10 mit der zugehörigen Korrelation des linearen Fits (b).

Der Trend der jährlichen Niederschlagsmenge hat im größten Teil des Arbeitsgebietes einen negativen Wert (Abbildung 5.4a). Ausnahmen bilden die Westküste, mit den Wüstengebieten der Namib und der Kap-Region, außerdem Gebiete im Nordosten Südafrikas und im Nordosten Simbabwes. Die Trends sind in beide Richtungen sehr stark ausgeprägt und entsprechen jeweils einer Erhöhung oder Verringerung der jährlichen Regenmenge von bis zu 50\% gegenüber dem Ausgangswert in 99/00. Die ohnehin trockenen Gebiete im südlichen Namibia und im westlichen Südafrika, aber vor allem auch weite Regionen des zentralen Südafrika und Lesothos sind stark von einem signifikanten (hohes $\mathrm{R}^{2}$ in Abbildung 
5.4b) Rückgang der Niederschlagsmenge betroffen, was sich nach einer ersten Einschätzung des Vegetationstrends in Abbildung 5.2 direkt auf das dortige Ökosystem auswirkt.

Die Entwicklung der mittleren Jahrestemperatur zeigt ein heterogeneres Bild (Abbildung 5.5a). Dennoch können weite Gebiete zusammengefasst werden, wie beispielsweise Botswana, für das nahezu gesamt ein negativer Temperaturtrend zu sehen ist. Mit Ausnahme des Nordens wird diese Region von Gebieten mit positivem Temperaturtrend umgeben, mit Spitzen von bis $\mathrm{zu}+30 \%$ (in Lesotho und dessen nordöstlicher Umgebung) gegenüber dem Wert zu Beginn der Betrachtung (1999/2000). Diese Spitzen entsprechen in den einzelnen Gebieten einer Erhöhung der mittleren Jahrestemperatur von einem halben Grad innerhalb der elf Jahre. Die Region in und um Lesotho zählt allerding zu den Gebieten mit den geringsten Jahresmitteltemperaturen von unter $10^{\circ} \mathrm{C}$ (vgl. Abbildung 2.2). Entlang der Küste im Westen und im Süden ergibt sich erneut ein negativer Temperaturtrend, nur im Osten des Arbeitsgebietes (Mosambik) zieht sich die Erwärmung an der Küste bis in den Norden. Die Güte der linearen Regression ist vor allem für die starken Temperaturtrends ( $> \pm 15 \%)$ relativ gut mit Werten größer 0.5 .

Eine Analyse der Anomalien der einzelnen Jahre des Niederschlags gegenüber dem Langzeitmittel von 1989 bis 2010 (ECMWF-Datenreihe) hat ergeben, dass die Perioden 99/00, 03/04 und 05/06 für das gesamte Gebiet sehr niederschlagsreich waren. Trockenperioden ergaben sich großflächig für 04/05, 06/07, 08/09 und 09/10. Dies entspricht dem Bild des vorwiegend negativen Niederschlagstrends in Abbildung 5.4a. Ein Vergleich der mittleren Jahrestemperaturen mit dem Langzeitmittel der Jahre 1989-2010 zeigte bis auf einzelne Gebiete (hauptsächlich in Botswana und Simbabwe) durchgehend positive Werte der Anomalien für die Perioden 99/00 bis 09/10. Demnach befindet sich die Region des südlichen Afrika generell über die letzten beiden Dekaden in einer Phase ansteigender Temperaturen. Über längere Zeiträume ist dieser Effekt sicherlich nicht zu vernachlässigen, da zum einen die Wachstumsperioden der Pflanzen (beispielsweise mit früherem Austreiben und späterem Laubabwurf) beeinflusst werden, zum anderen die photosynthetische Aktivität bei zu stark ansteigenden Temperaturen durch Limitierung der Photosynthese selbst, aber auch durch sinkende Wasserverfügbarkeit aufgrund hoher Evaporationsraten reduziert wird. Für eine bessere Übersichtlichkeit in der folgenden Indexierung der Degradation werden aus den klimatischen Entwicklungen nur der Anstieg der Temperatur und der Rückgang der Niederschlagsmenge als relevant betrachtet. Diese Annahme stützt sich auf die Ergebnisse der Sensitivitätsstudie (Kapitel 3.1) und die Verteilung der mittleren Jahrestemperaturen und Niederschlagsmengen innerhalb des Arbeitsgebietes (Abbildung 2.2). Außerdem werden nur Trends mit einer Korrelation von mehr als 0.5 berücksichtigt. Im Folgenden wird demnach ein klimatischer Einfluss angenommen, wenn die Temperatur über den Zeitraum 
um mehr als 10\% angestiegen ist oder sich die Niederschlagsmenge um mehr als $30 \%$ reduziert hat. Dies entspricht je nach Region einem Temperaturanstieg um ein bis zwei Grad Celsius und einem Rückgang der Niederschlagsmenge um 60 bis 420 mm.

\subsection{Indexierung der Landdegradation}

Die Bewertung der Landdegradation aus den Modellergebnissen von BETHY/DLR soll die vorher diskutierten, klimatischen Bedingungen berücksichtigen. Dies ist hilfreich bei der Einschätzung, wie sehr die Bewirtschaftung der Vegetation durch den Menschen zu einem Rückgang der vegetativen Produktivität geführt hat. Vor allem das Busch- und Grasland wird im südlichen Afrika als intensive Weidefläche genutzt (Ross, 1999; Fox und Rowntree, 2001) und wird in der nachfolgenden Betrachtung einen besonderen Stellenwert einnehmen.

Zur Unterscheidung der klimatisch und nicht-klimatisch bedingten Trends erfolgt eine Einteilung des in Abbildung 5.2 vorgestellten NPP-Trends in mehrere Kategorien (Abbildung 5.6). Dies erfolgt zunächst über die Temperatur- und Niederschlags-Trends aus Kapitel 5.2. Liegt der Trend des Niederschlags unterhalb von $-30 \%$ wird das Pixel in die Kategorie B (braun) eingeordnet. Bei einem Temperatur-Trend von mehr als $+10 \%$ erfolgt die Zuordnung zu Kategorie C (rot). Sind beide Bedingungen erfüllt, kann von einer ausgeprägten Änderung der Klimabedingungen ausgegangen werden, die ausreicht, die Entwicklung der Vegetation entscheidend zu beeinflussen. Dies wird in Kategorie D (blau) eingeteilt. Tritt weder ein signifikanter negativer Niederschlags-Trend noch ein signifikanter positiver Temperatur-Trend auf, erfolgt die Einordnung in Kategorie A (grün), in denen Regionen zusammengefasst werden, die demnach durch den menschlichen Einfluss einen Rückgang der vegetativen Produktivität erfahren. Zur Bewertung des Grades der Reduzierung werden die einzelnen Kategorien in weitere Klassen unterteilt. Ein Rückgang der Produktivität um bis zu 25\% wird mit dem Index 1 bezeichnet. Liegt die Reduzierung zwischen $25 \%$ und 50\% wird der Index 2 zugewiesen, zwischen 50\% und 75\% der Index 3 und ab 75\% der Index 4 (Abbildung 5.6). Die Einteilung des Degradationsindexes ist in Tabelle $5.1 \mathrm{zu}-$ sammengefasst.

Einen deutlichen Einfluss einer Klimaänderung erfahren weite Gebiete der Namib im südlichen Namibia und des nordwestlichen Südafrikas (klassifiziert als Grasland), für die großflächig ein Rückgang von bis zu 50\% (Index D2) ermittelt wird, der lokal auch auf über 75\% (Index D4) ansteigt. Solche Gebiete sind auch in Lesotho, Swasiland und dem östlichen Simbabwe ersichtlich, wobei hier meist ein Index von D4 erreicht wird. Diese sind im Wesentlichen mit den bereits diskutierten Bodentypen verbunden, die generell schlechte Wasserspeichereigenschaften besitzen. Das wird auch bei Betrachtung der Kate- 
gorie B deutlich, deren höchste Werte (Index B4) ebenfalls für diese Bodentypen auftreten. Dabei spiegelt sich die Problematik wieder, dass sich bei ohnehin schlechten Bodenbedingungen der Wassereintrag durch den Niederschlag zunehmend reduziert.

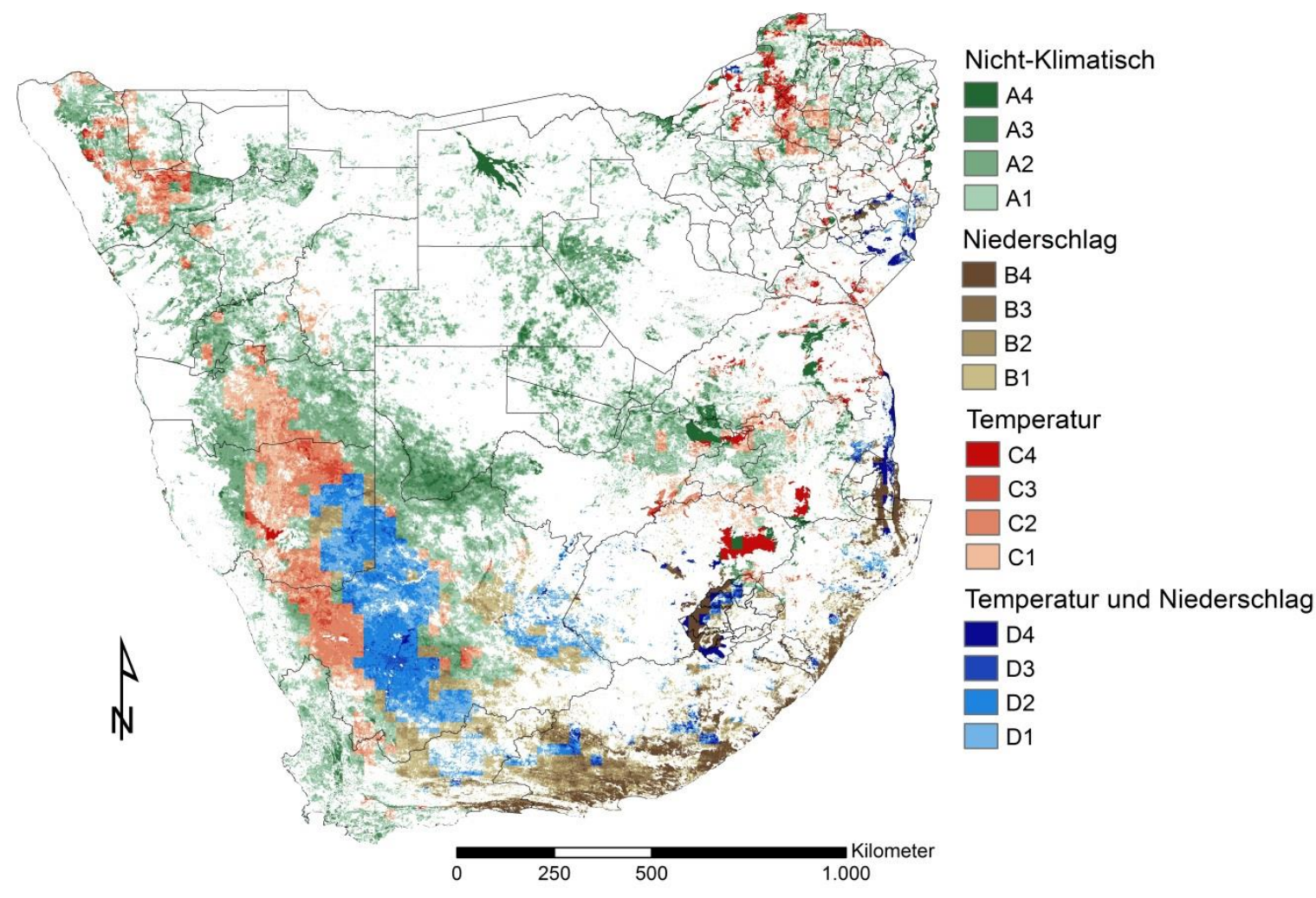

Abbildung 5.6: Degradationsindex des Arbeitsgebietes mit den Unterteilungen in die Kategorien A: nicht-klimatisch bedingt (grün), B: signifikanter Rückgang des Niederschlags (braun), C: signifikante Temperaturzunahme (rot) und D: kombinierte Temperaturzunahme und Niederschlagsrückgang (blau). Die Zahlenzusätze bezeichnen den Grad des NPP-Rückgangs: 1: 0 bis 25\%; 2: 25\% bis 50\%; 3 : $50 \%$ bis $75 \%$; 4 : über $75 \%$.

Tabelle 5.1: Klassifizierung des Degradationsindexes mit der Farbkodierung aus Abbildung 5.6. $\triangle$ NPP ist der NPP-Trend, $\Delta P$ der Niederschlags-Trend und $\Delta \mathrm{T}$ der Temperaturtrend über den Betrachtungszeitraum $99 / 00$ bis $09 / 10$.

\begin{tabular}{rcccc}
\hline \multicolumn{1}{c}{$\Delta$ NPP } & $\begin{array}{c}\text { nicht- } \\
\text { klimatisch }\end{array}$ & $\Delta \mathrm{P}<-30 \%$ & $\Delta \mathrm{T}>+10 \%$ & $\begin{array}{c}\Delta \mathrm{P}<-30 \% \& \\
\Delta \mathrm{T}>+10 \%\end{array}$ \\
\hline$<-75 \%$ & A4 & B4 & C4 & D4 \\
$-50 \%--75 \%$ & A3 & B3 & C3 & D3 \\
$-25 \%--50 \%$ & A2 & B2 & C2 & D2 \\
$0 \%--25 \%$ & A1 & B1 & C1 & D1 \\
\hline
\end{tabular}


Außerdem sind in der Kategorie B die Waldgebiete an der Südküste Südafrikas vertreten, ebenfalls mit Verlusten von bis zu 75\% und darüber hinaus. Hohe Temperaturzunahmen haben in weiteren Gebieten der Namib, hauptsächlich in Namibia, aber auch im nordwestlichen Südafrika zu einem NPP-Rückgang geführt. Hier sind hauptsächlich die Indizes C2 und C3 zu finden, lokal sind allerdings auch Rückgänge von über 75\% (Index C4) erkennbar. Gleiches gilt auch für die Region westlich der Etosha-Pfanne im Norden Namibias (klassifiziert als Busch- und Grasland), und im Norden Simbabwes (klassifiziert als Ackerland).

Schließlich bleiben die Regionen, für die kein signifikanter Trend der klimatischen Bedingungen ermittelt wurde (Kategorie A). Zwar wird nicht nur für diese Regionen eine Nutzung durch den Menschen angenommen, doch wird davon ausgegangen, dass hier alleine die Bewirtschaftung für einen Rückgang der vegetativen Produktivität verantwortlich ist. Dies zeigt sich für alle ermittelten Gebiete in Botswana, mit dem höchsten Verlust (Index A4) für das Okavango-Delta, in dem intensiv Landwirtschaft betrieben wird. In Südafrika sind ebenfalls die landwirtschaftlichen Flächen um die Metropolen Johannesburg und Pretoria aber auch in der Kap-Region betroffen. Außerdem ergeben sich Verlustraten von bis zu 75\% (Index A3) für die Grasland-Flächen der Kalahari in der Grenzregion zwischen Botswana und Südafrika. Teilweise werden auch hier Raten von über 75\% (Index A4) gefunden. Wie bereits erwähnt wird hier der Verlust ausschließlich auf die intensive Weideland-Nutzung zurückgeführt. Für die angrenzenden Gebiete der Kategorie B, C und D in Namibia und Südafrika kann eine Kombination aus der Überbeanspruchung durch den Menschen und den Veränderungen der klimatischen Bedingungen angenommen werden, die zu einem Rückgang der NPP führt.

Zur Bewertung der Ergebnisse stehen leider keine überregionalen Vergleichsstudien zur Verfügung über die das gesamte Ergebnis mit weiteren, über das Arbeitsgebiet konsistenten Untersuchungen zur Landdegradation, verglichen werden kann. Allerdings besteht die Möglichkeit das Produkt mit Arbeiten zu vergleichen, die sich auf eine lokale bis regionale Ausdehnung beschränken. Dafür wurden bis auf Swasiland für alle Länder des Untersuchungsgebietes veröffentlichte Studien gefunden, die im Folgenden einzeln betrachtet werden.

\subsubsection{Namibia}

Strohbach (2001) berichtet über Degradationsprozesse in der nördlichen Oshikoto-Region Namibias und der Zentralregion um die Hauptstadt Windhoek. Die Studie verwendet den

Degradationsgradienten nach Bosch und Janse van Rensburg (1987) und Bosch et al. (1987). Beobachtet wurde dabei in erster Linie meist der Übergang einer grasbedeckten 
Landschaft zu hölzerner Savannen-Vegetation durch intensive Weidelandnutzung. Aber auch der Verlust an bewachsenen Flächen spielt in vielen Regionen eine wichtige Rolle, wie beispielsweise in der nördlichen Oshikoto-Region westlich der Etosha-Pfanne oder der Oshana-Ebene weiter im Norden (Strohbach 2000a, 2000b). Für beide Regionen wird auch ein Degradationsindex der Kategorie A2 bis A3, mit landwirtschaftlichen Flächen im Norden und Weideland im westlichen Gebiet bestimmt (Abbildung 5.7). Für Gebiete deren Vegetation durch andere Arten des Bewuchses ersetzt wurde ist meist auch keine Änderung anhand des Index ersichtlich, wie in der Okatope-Region nordöstlich der Etosha-Pfanne (Strohbach, 2001).

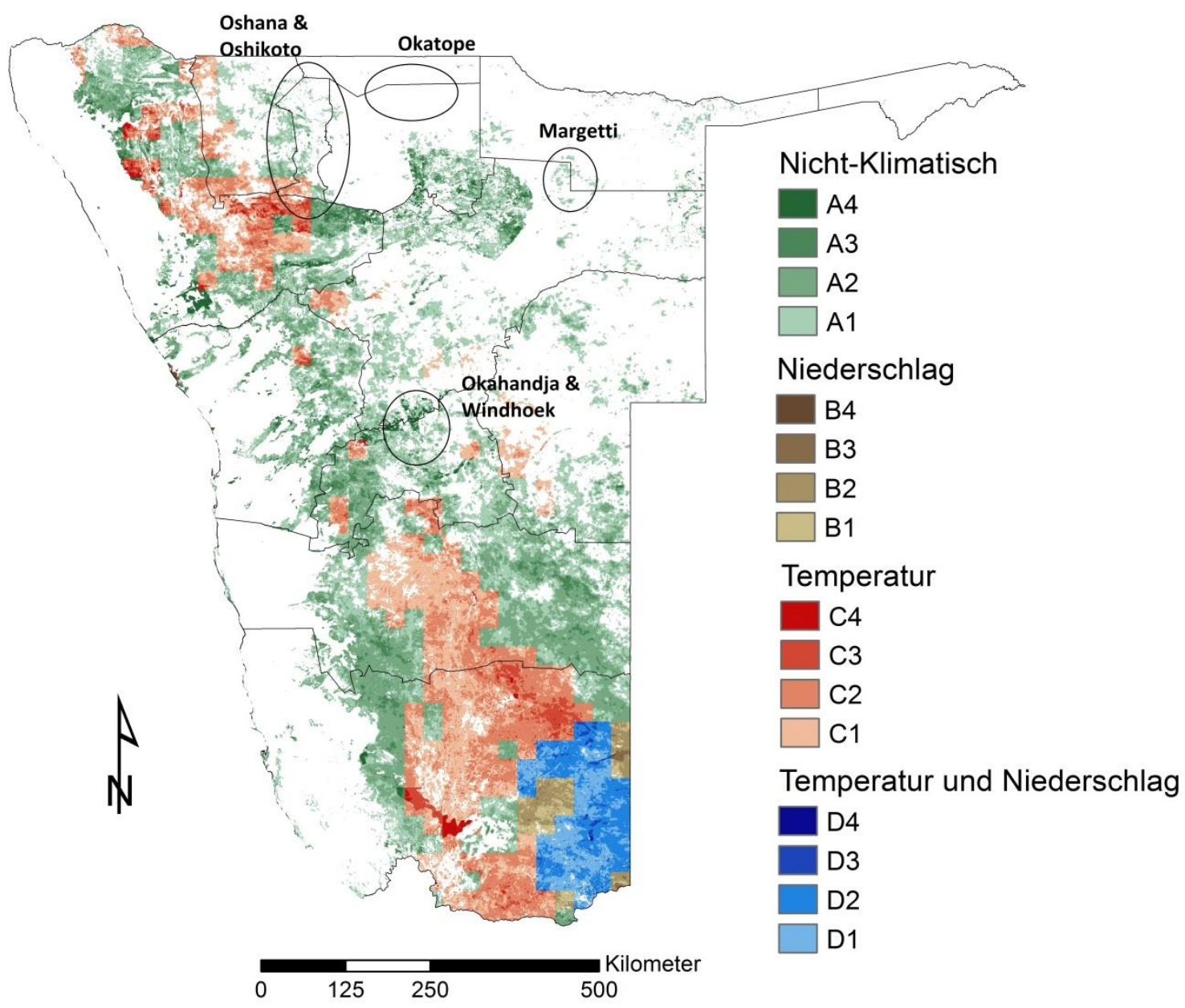

Abbildung 5.7: Degradationsindex für Namibia mit den Vergleichsregionen (schwarze Markierungen).

In der Mangetti-Region wiederum wird ein Verlust an Buschland-Vegetation beschrieben, der mit einem Index der Kategorie A2 in der Region bestätigt werden kann. Auch hier scheint hauptsächlich die intensive Nutzung als Weideland für den Verlust an Produktivität verantwortlich zu sein. 
In Zentralnamibia wird für die Distrikte Okahandja und Windhoek ein starker Rückgang der Graslandvegetation innerhalb des Zeitraumes 1985 bis 2001 berichtet, in Teilen sogar der komplette Verlust der Grasbedeckung (Strohbach, 2001). Dies gilt für sonst gute klimatische Bedingungen, daher ergibt sich für die Entwicklung im Zeitraum von 1999 bis 2010 für beide Gebiete ein Degradationsindex der Kategorie A mit einer Verminderung der Produktivität von mehr als 75\% (Index A4). Dies ist ein deutliches Indiz für die Beeinflussung der natürlichen Vegetation durch die intensive Viehhaltung.

\subsubsection{Botswana}

Weniger als 5\% der Fläche Botswanas eignen sich für regenbewässerten Ackerbau. Sonst (95\%) wird im ganzen Land Viehwirtschaft betrieben, davon 71\% durch Kommunen und Stämme, 23\% ist Staatsgebiet und die restlichen 6\% kommerzielle Farmen. 18\% der Fläche ist Weideland und gleichzeitig ausgeschrieben als Nationalparks und Wildreservate, wodurch sich zunehmend Konflikte ergeben (Darkoh, 1999). Zwar sind die ariden und semiariden Ökosysteme wie in der Kalahari offensichtlich durch die Anpassungen an die Bedingungen über mehrere Jahrzehnte hinweg extrem belastbar. Allerdings sind sie den immer weiter zunehmenden Beanspruchungen durch den Menschen, wie beispielsweise durch intensive Weidewirtschaft, Holzgewinnung oder Ackerbau nicht gewachsen. Dies sind vor allem die direkten Auswirkungen der immer schneller wachsenden Bevölkerung in den Regionen, die immer größere Viehherden und intensiveren Ackerbau bedingt.

Auch innerhalb des letzten Jahrzehnts scheint diese Problematik vorherrschend zu sein, nachdem alle ermittelten Regionen des Landes der Kategorie A zugeordnet sind (Abbildung 5.8), und demnach keine besonderen klimatischen Veränderungen stattgefunden haben. Van Vegten (1981) berichtete bereits von einer Reduzierung der Grasbedeckung im südlichen Kgatleng-Distrikt von 6-15\% auf 0-2\% aufgrund der dortigen Viehhaltung. Aus den Modellergebnissen ergibt sich ein Degradationsindex von A2 bis A3 für Ackerund Grasland (Abbildung 5.8). Vergleichbar hoch (stellenweise A4), aber weiträumiger ausgeprägt, ist der Degradationsgrad in der Boteti-Region, westlich der MakgadikgadiSalzpfannen. Für dieses Gebiet wurden von Darkoh (1999) deutliche Anzeichen für Landdegradation und Desertifikation über 30 Jahre, von Ende der 1960er bis Ende der 1990er Jahre, nachgewiesen. Nach den Erkenntnissen aus den Modellergebnissen sind diese auch bis heute noch zu erkennen.

De Queiroz (1993) stellte die besondere Bedeutung der industriellen Viehzucht für die Beanspruchung von Gras- und Buschland heraus. Durch Studien innerhalb des KhutseWildreservats in der Kalahari Botswanas konnte gezeigt werden, dass der Rückgang der dortigen Vegetation in direktem Zusammenhang mit der intensiven Beweidung gebracht 
werden kann. Gleiches gilt für die Bewertung der Vegetationsentwicklung der nordöstlich gelegenen Uwe-Aboo-Farm durch Dougill et al. (1999). Hier wird hauptsächlich ein Verlust an Graslandvegetation über den Zeitraum der 1990er Jahre verzeichnet, der stellenweise durch Büsche ausgeglichen wird. Für beide heute noch bestehenden Farmen wird auch im Zeitraum 1999 bis 2010 ein hoher Degradationsindex von A2 bis A3 bei zugrundeliegender Graslandvegetation erreicht.

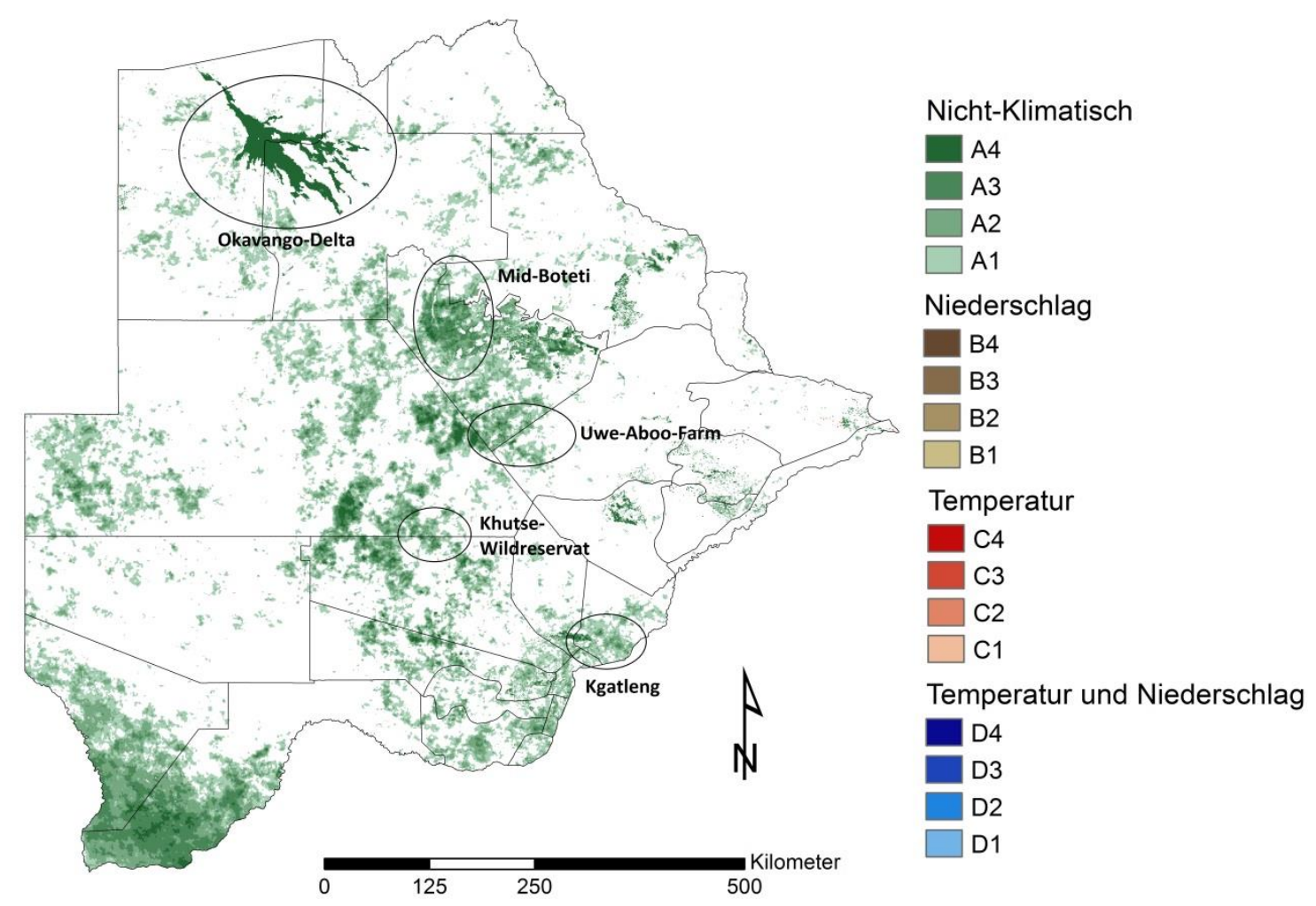

Abbildung 5.8: Degradationsindex für Botswana mit den Vergleichsregionen (schwarze Markierungen).

Eine Besonderheit in Botswana ist sicherlich die regelmäßig überflutete Sumpfregion des Okavango-Deltas. Dieses Gebiet ist nach den Ergebnissen der NPP aus dem Modell mit dem höchsten Index A4 gekennzeichnet. Als Vergleich dient die Arbeit von Hamandawana et al. (2007) zur Bewertung der Landdegradation im Okavango-Delta und dessen Umgebung im Zeitraum von 1860 bis 2001. Als Hilfsmittel wurden historische Aufzeichnungen, Feldbeobachtungen, mündliche Befragungen der Bewohner über die Einschätzung der Umweltveränderungen und letztlich Satellitenbilder der Jahre 1967, 1989, 1994 und 2001 von Corona und Landsat verwendet. Bereits aus den Befragungen der Bevölkerung ergibt 
sich die Einschätzung einer rückläufigen Menge an Oberflächenwasser, einem Rückgang des Grundwassers und dem Verlust an Weideland. Als Grund für die Entwicklungen wird ein negativer Trend der Niederschlagsmengen und Verluste durch Überschwemmungen angenommen, zusätzlich aber auch die steigende Beanspruchung des Landes durch den Menschen (Hamandawana et al. 2005). Auch die Analysen der Satellitenbilder zeigten eine Verschlechterung der Umweltbedingungen, wobei bereits bis 1989 ein Rückgang des Oberflächenwassers um mehr als $12 \%$ verzeichnet wird. Zwischen 1989 und 2001 wird eine Verminderung des Baumbewuchses um $5 \%$ verzeichnet, wogegen Buschland sich im Aufwärtstrend befindet (6.6\% seit 1967). Deutlich ist dagegen der Rückgang der Graslandvegetation um etwa $44 \%$ im Zeitraum von 1967 bis 2001. Hauptgrund hierfür ist zunächst der Zuwachs der Bevölkerung in dem Gebiet um mehr als 50 \%, alleine zwischen 1981 und 2001, und damit verbunden die Zunahme der Viehhaltung (Hamandawana et al. 2005). Verstärkt wird die Entwicklung schließlich mit der Abnahme der Niederschlagsmengen innerhalb des Zeitraumes. Die Ergebnisse der Modellrechnungen zeigen diese Trends auch für die anschließende Dekade in ebenso drastischer Weise.

\subsubsection{Simbabwe}

In Simbabwe sind über $70 \%$ der Bevölkerung von der Viehzucht abhängig, wobei sich die Fläche zu jeweils etwa $40 \%$ des gesamten Landes auf kommunale und kommerzielle Bewirtschaftung aufteilt. Etwa $80 \%$ der kommunalen Weideflächen gelten als degradiert (Scoones, 1992). Als Beispiel dient der Buhera-Distrikt im Einzugsgebiet des Flusses Save im Osten des Landes aufgeführt. Die Vegetation verteilt sich zu über 70\% auf bewirtschaftete Flächen und zu knapp $28 \%$ auf Waldgebiete, wobei diese zunehmend dem Weideland weichen müssen. Mambo und Archer (2007) konnten mit Hilfe von NDVI-Karten der Jahre 1992 und 2002 die Region in vier Grade der Degradation (von gering bis sehr hoch) einteilen. Betroffen ist der Norden des Distrikts sowie vereinzelte Flächen im Süden, wobei dem Norden großflächig ein mittlerer Degradationsgrad zugewiesen wird. Der Süden ist eingeteilt in ein Gebiet hoher Degradation im Westen, eine heterogene Region im Osten mit teilweise mittlerer, teilweise sehr hoher Degradation und schließlich ganz im Süden sehr vereinzelte kleinere Flächen sehr hoher Degradation (entsprechend den Markierungen in Abbildung 5.9). Aus den Modellergebnissen ergibt sich für das Gebiet im Norden des Buhera-Distrikts (klassifiziert als Buschland) eine größere Region mit einem Index von A4 bzw. C4. Die Region erfährt also teilweise einen starken Anstieg der Jahresmitteltemperatur, der für die Entwicklung der Vegetation mit verantwortlich sein kann. Die Region im Süden ist hauptsächlich als Ackerland klassifiziert und teilt sich ebenfalls auf in ein Gebiet im Westen mit einem Index von C4, eines im Osten ebenfalls mit einem Index von C4 und eines im äußersten Süden in dem vereinzelt ein Index von D1 zu finden ist. Im Gegensatz 
zur Studie von Mambo und Archer (2007) wird im Nordosten Buheras ein Gebiet, hauptsächlich Ackerland, mit einem Degradationsindex von C3 bis C4 angezeigt, das einen starken positiven Temperatur-Trend erfährt.

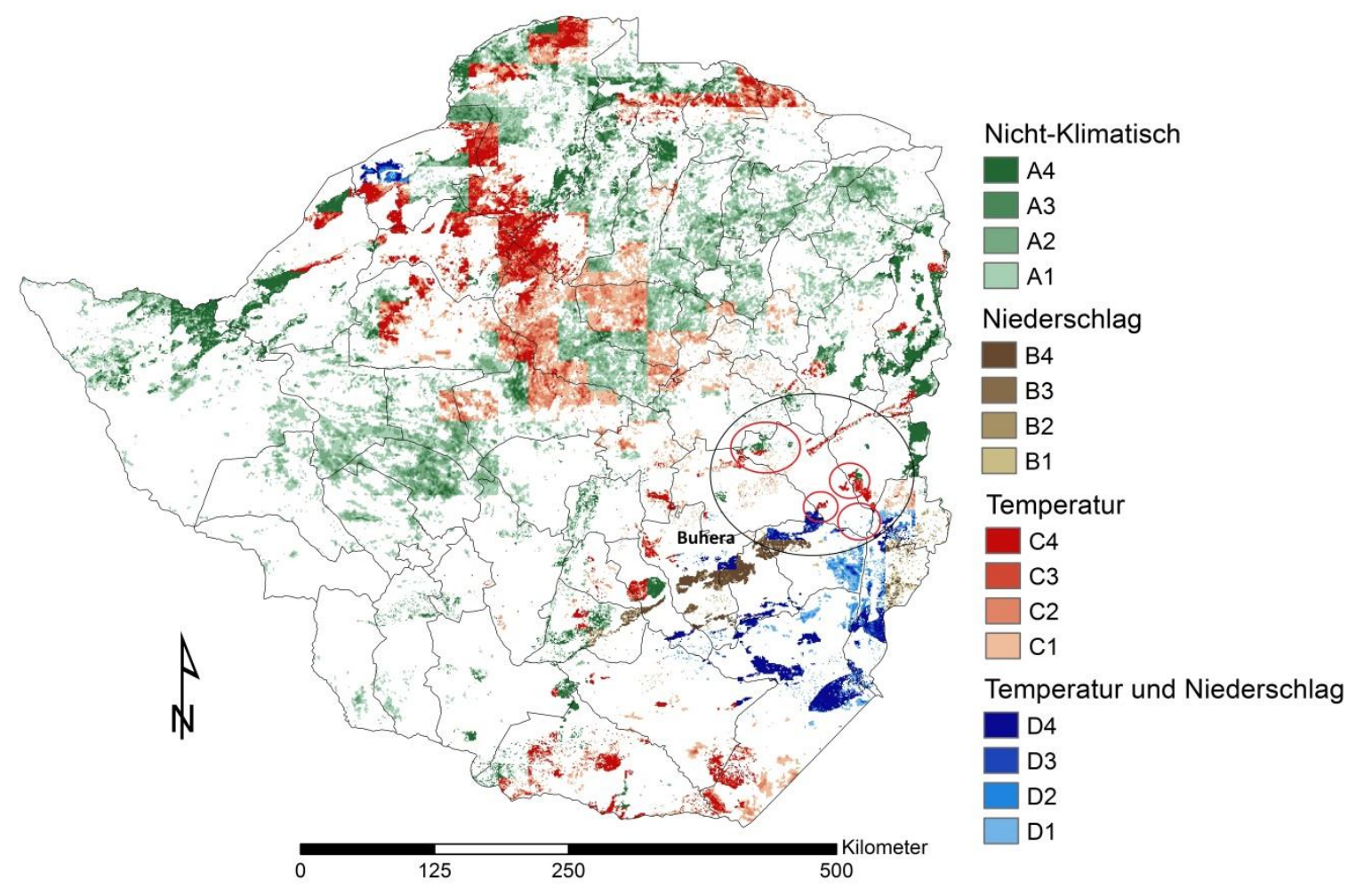

Abbildung 5.9: Degradationsindex für Simbabwe mit der Vergleichsregion Buhera (schwarze Markierung). In der Region sind die von Mambo und Archer (2007) als degradiert identifizierten Gebiete markiert (rote Markierungen).

\subsubsection{Südafrika}

Der größte Anteil der Fläche in Südafrika wird für die Landwirtschaft genutzt. Diese Nutzung teilt sich auf in etwa $13 \%$ für den Anbau von Kulturpflanzen und etwa $87 \%$ für Weideland und Viehhaltung (National Land Cover - NLC, 2009). Die nationale Landbedeckungsklassifikation, erstellt aus Landsat-Daten von 2000 bis 2002, gibt außerdem insgesamt etwa $3 \%$ der gesamten Fläche Südafrikas als von Landdegradation betroffene Gebiete an. In Anbetracht der vorausgehenden Ergebnisse der benachbarten Länder erscheint dies allerdings sehr gering, besonders unter Beachtung, dass über $90 \%$ der Fläche Südafrikas mit semi-ariden Klima als Trockengebiet klassifiziert wird (Hoffman et al., 1999) und dadurch stark anfällig für Degradation und Desertifikation ist. Allerdings wer- 
den in der NLC solche Gebiete als degradiert klassifiziert, deren Vegetationsbedeckung geringer ist als die der umgebenden Regionen (Thompson, 1996). Demnach werden sich erwartungsgemäß durch die hier vorgestellte Methode wesentlich größere Flächen der Degradation ergeben.

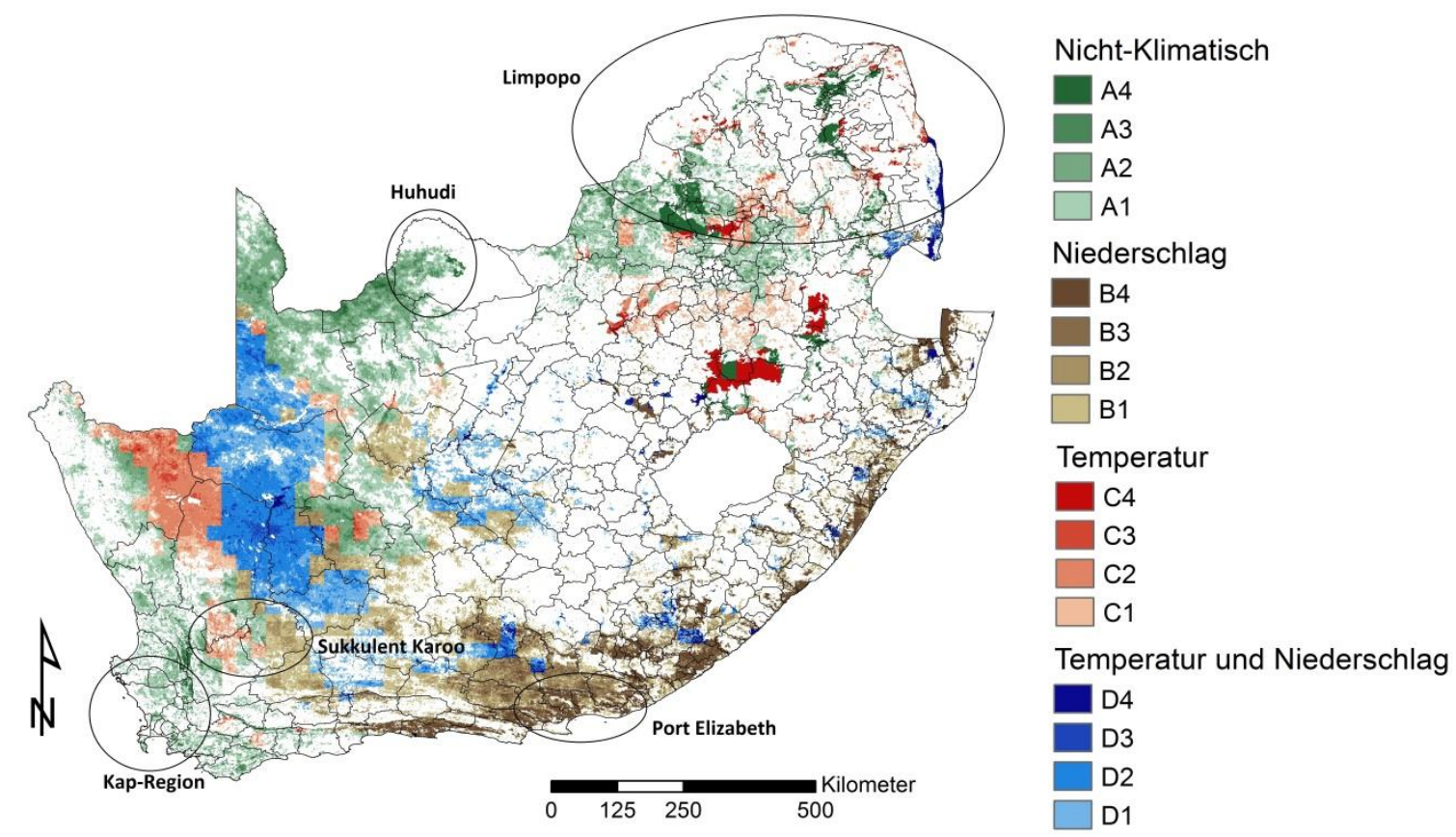

Abbildung 5.10: Degradationsindex für Südafrika mit den Vergleichsregionen (schwarze Markierungen).

Übereinstimmung zwischen den beiden Produkten ergibt sich in der Provinz Nord-West für die Distrikte Huhundi, Kuruman und Kudumane nahe der Grenze zu Botswana (Abbildung 5.10). Die als Grasland klassifizierte Region wird mit einem Degradationsindex von A2 bis A3 bewertet, im zentralen Huhundi auch bis zu A4. Schlechte Übereinstimmung ergibt sich für die Provinz Limpopo, in der zwar weite Gebiete von der NLC als degradiert definiert sind, diese aber nicht durch den Degradationsindex wiedergegeben werden. Umgekehrt erhalten große Gebiete hohe Indizes, die von der NLC als unbeeinflusste Vegetation eingestuft werden. Weitere Hotspots nach der NLC liegen im Ostkap, nordöstlich von Port Elizabeth (als Waldflächen klassifiziert) und im Westkap in der Kapregion, östlich von Kapstadt(Ackerland). Für beide Regionen ergibt sich eine gute Übereinstimmung mit hohen Indizes von B3 bis B4 bei Port Elisabeth und A1 bis A2 in der Kap-Region. In der Region um Port Elizabeth folgt der Trend der Vegetation dem der Niederschlagsmenge, in der Kap-Region sind allerdings keine Einflüsse der klimatischen Entwicklung zu erkennen. 


\subsubsection{Lesotho}

Majara (2005) bewertete den Degradationsgrad von Lesotho ebenfalls mit Hilfe von NDVI-Karten der Jahre 1989 und 1999, wobei der höchste Grad für die MokhotlongRegion im Osten und Berea-Region im Nordwesten ermittelt wird, hohe Grade zeigen sich auch für die Butha-Buthe-Region im Norden und die Regionen Mohale's Hoek und Quithing im Süden.

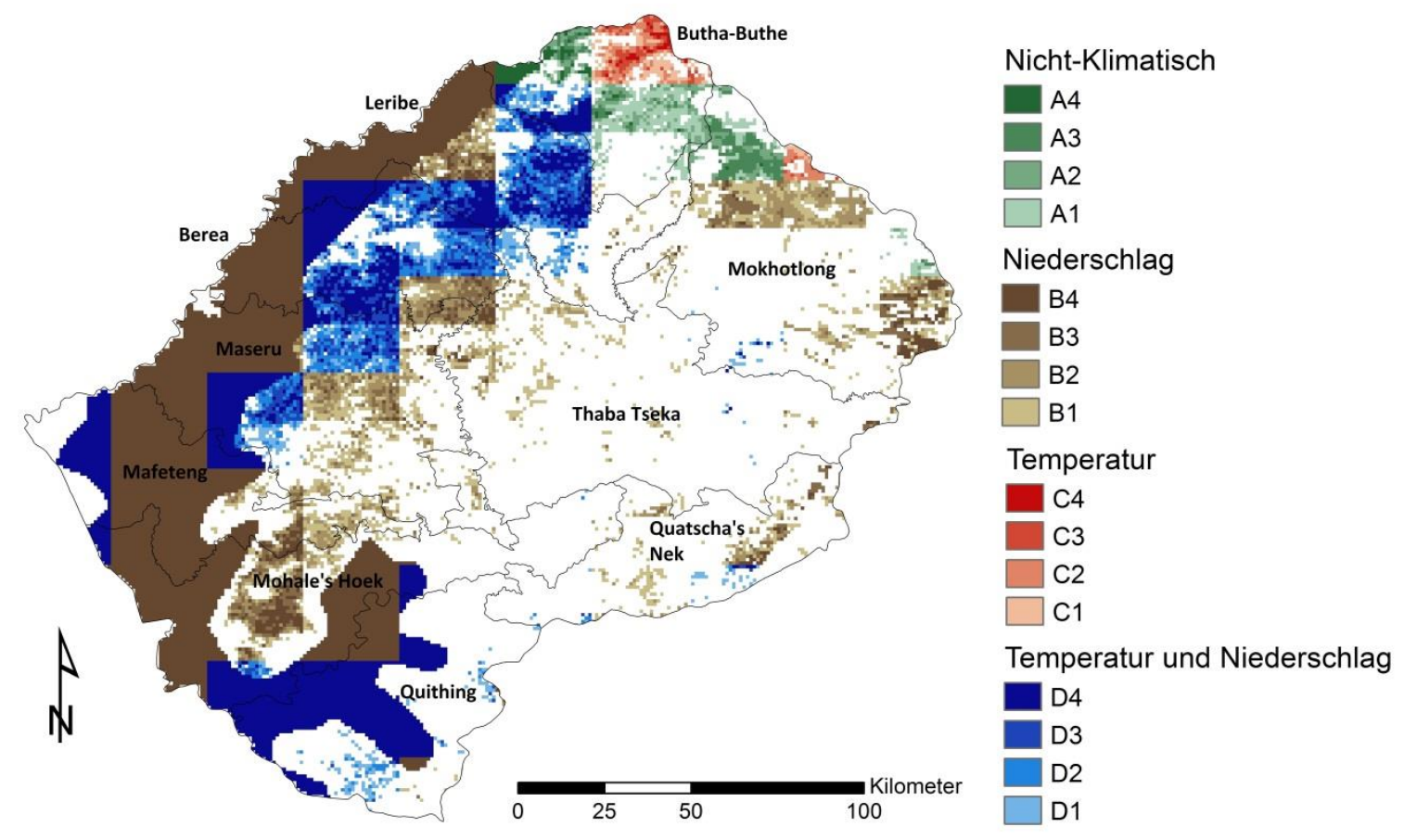

Abbildung 5.11: Degradationsindex für Lesotho mit den einzelnen Regionen.

Zum Vergleich zeigt die Mokhotlong-Region nur im Norden und im Osten einen Index von 2 bis 3 in den Kategorien A, B und C. Ansonsten wird der Degradationsindex in Lesotho durch die Struktur des Bodentyps entlang der Grenze im Nord- und Südwesten geprägt. Dadurch ergibt sich für die entsprechenden Regionen ein hoher Index von 4, hauptsächlich durch einen starken Rückgang der Niederschlagsmenge verursacht (Kategorie B), teilweise aber auch durch eine zusätzliche Erhöhung der Jahrestemperatur (Kategorie D). Dies gilt auch für Distrikte die in der Studie von Majara (2005) mit geringem Degradationsgrad angegeben werden. Für die Butha-Buthe-Region ergibt sich dagegen eine gute Übereinstimmung von hoher Degradation und dem häufigsten Degradationsgrad von 2 in den Kategorien A, C und D. 
Aus den verschiedenen Studien geht mehrheitlich eine Übereinstimmung der regionalen Bewertungen des Degradationszustandes mit dem vorgestellten Degradationsindex hervor. Kritisch ist allerdings, dass die Zeitpunkte der meisten der verwendeten Arbeiten nicht innerhalb des Modellierungszeitraumes liegen. Auch zwischen den Skalen der verschiedenen Untersuchungen und des Degradationsindexes besteht ein signifikanter Unterschied. Dennoch kann aufgrund der Häufigkeit an deutlichen Übereinstimmungen auf kleinen Skalen die Übertragbarkeit der Ergebnisse auf die großflächige Darstellung der Vegetationsentwicklung angenommen werden. Lokale Gegebenheiten können allein aufgrund der Auflösung der Modellergebnisse nicht im Detail wiedergegeben werden. Dies ist allerdings auch nicht Ziel der vorgestellten Methode. Größerer Wert wird auf die Möglichkeit der großskaligen Bewertung des Vegetationszustandes über längere Zeiträume gelegt.

Die Vergleiche haben auch gezeigt, dass Landdegradation in Form des Verlustes einer Vegetationsbedeckung zugunsten eines anderen Vegetationstyps nicht erfasst werden kann. Ein Beispiel hierfür ist der Rückgang des Graslandbewuchses in weiten Teilen der Kalahari in Botswana und Namibia und die gleichzeitige Zunahme an holziger Vegetation, meist Buschland oder kleinere Bäume (Dougill und Cox, 1993). Da durch diesen Übergang nicht zwangsläufig eine Verminderung der Produktivität verbunden ist, werden solche, ebenfalls in vielen Arbeiten als Degradation bezeichneten Variationen in der Vegetationsbedeckung, nicht durch den hier vorgestellten Index erfasst. 



\section{$6 \quad$ Zusammenfassung}

Die Produktivität der Vegetation hat für die dynamische Beschreibung von Ökosystemen unmittelbare Bedeutung. Sie spiegelt anthropogene Einflüsse sowie Variationen in den klimatischen Bedingungen wieder. Über die Art der Bewirtschaftung sowie über die Entwicklung der natürlichen Bedingungen wird der Grad der photosynthetischen Produktivität direkt beeinflusst. Besondere Bedeutung kommt den Auswirkungen in Ökosystemen zu, deren äußere Bedingungen ohnehin bereits besondere Ansprüche an die Vegetation stellen. Dazu gehören auch die Trockengebiete der Erde, wie weite Teile des südlichen Afrikas, auf denen in dieser Arbeit der Fokus lag. Die Beobachtung der ariden und semi-ariden Regionen im Hinblick auf die Entwicklung der Pflanzenwelt spielt eine wichtige Rolle in der Bewertung der Ökosysteme als relevante Kohlenstoffsenken in der Region. Seltene Niederschlagsereignisse mit geringen Regenmengen stellen erschwerte Bedingungen für die Pflanzenwelt dar. Die Überbeanspruchung der Vegetation als Weide- und Ackerland erhöht den Druck auf die Ökosysteme zusätzlich. Beides führt im schlimmsten Falle zu einem Verlust der vegetativen Bedeckung und zur Degradation weiter Gebiete. Dadurch kommt es in den Regionen Südafrikas zu einer weiteren Ausbreitung der Wüstengebiete. Einfluss auf die meist kommunal oder privat organisierte Landbewirtschaftung kann auf solch großen Skalen nur die Politik nehmen. Für die Farmer müssen Anreize geschaffen werden, die Größe ihrer Herden zu reduzieren, ohne dass sie einen wirtschaftlichen Verlust erleiden. Dafür müssen allerdings zunächst die tatsächlich gefährdeten Regionen identifiziert werden. Für diese Notwendigkeit fehlte bisher ein objektiver Ansatz, der es ermöglicht, eine Bewertung der Situation auf nationaler Skala zu erstellen und zusätzlich die entsprechende Auflösung besitzt, um Aussagen auf regionaler Ebene treffen zu können.

Die vorliegende Arbeit soll einen Beitrag zum Ausgleich dieser Wissenslücke leisten. Ziel war es aufgrund von Modellergebnissen zur Produktivität der Vegetation im südlichen Afrika eine Bewertung der Entwicklung der Pflanzen über einen längeren Zeitraum zu erstellen. Dafür wurde das SVAT-Modell BETHY/DLR verwendet, dessen primäres Ergebnis die NPP ist. Als Datengrundlage des Modells dienen meteorologische Zeitreihen von Temperatur, Niederschlag, Wolkenbedeckung und Windgeschwindigkeit aus der ERA-InterimDatenbank des ECMWF. Außerdem wird die Landbedeckungsklassifikation der GLC2000 mit einer Auflösung von $1 \mathrm{~km}$ zur Unterscheidung der Vegetationstypen verwendet. Mit gleicher Auflösung geht schließlich der LAI, als Information über die saisonale Entwicklung der Vegetation, ein. Diese Datenreihe bestimmt letztlich die Auflösung der Modellergebnisse. Die Zeitreihen des LAI benötigen eine Bearbeitung bevor sie als Eingangsdaten für das Modell verwendet werden können, da sie Datenlücken als Folge von Wolkenbedeckung während der Datenaufnahme enthalten. Dafür wird eine mittlere, pflanzenspezifi- 
sche Phänologie des regionalen Ökosystems berechnet und zum Auffüllen großer Lücken verwendet. Zur Interpolation kleinerer Lücken und zur Glättung der LAI-Zeitreihen findet die Methode der Harmonischen Analyse Anwendung. Mit deren Hilfe werden die dominanten harmonischen Schwingungen des LAI-Eingangssignals ermittelt, aus denen lückenlose Datenreihen zur Repräsentation der saisonalen phänologischen Entwicklung aufsummiert werden. Ein Vergleich der Ergebnisse mit Bodenmessungen des LAI ergab eine gute Übereinstimmung mit einem $\mathrm{R}^{2}$ von 0.83 und einem RMSE von 0.88. Als Nebenprodukt dieser Arbeit konnte mit dem Verfahren als erster seiner Art ein globaler, räumlich und zeitlich kontinuierlicher Datensatz der SPOT-LAI-Zeitreihen von 1999 bis 2010 erstellt werden, der über das Webportal des World Data Center for Remote Sensing of the Atmosphere WDC-RSAT frei verfügbar ist.

Da das Modell BETHY/DLR im Rahmen dieser Arbeit zum ersten Mal für aride Gebiete angewandt wurde, mussten vereinzelt Anpassungen an die entsprechenden Gegebenheiten vorgenommen werden. Zum einen wurden die pflanzenphysiologischen Parameter der Elektronentransportrate und der Carboxylierungsrate durch Literaturwerte für regionale Vegetationstypen ersetzt, zum anderen wurde die Parametrisierung des Bodenmodells durch eine Formulierung zur Berücksichtigung von Trockenstress erweitert. Dadurch wird die Gegebenheit berücksichtigt, dass die Pflanzen bei zu geringen Niederschlagsmengen und folglich bei zu geringem Bodenwassergehalt kein Wasser über die Wurzeln aufnehmen können. Die Ergebnisse dieser Arbeit haben gezeigt, dass dies im südlichen Afrika für bestimmte Bodentypen und Regionen kein unerheblicher Faktor ist.

Als ein wesentlicher Punkt wurde die Qualität der Eingangsdaten durch Vergleiche mit Stations- und Bodenmessungen bestimmt. Zusätzlich konnte durch eine Sensitivitätsstudie die für das Modell wichtigsten Parameter identifiziert werden. Daraus ergeben sich für den LAI und die Verteilung der Vegetationstypen über die Landbedeckungsklassifizierung die stärksten Abhängigkeiten des Modells gegenüber Variationen in den Parametern. Für den LAI konnte ein $\mathrm{R}^{2}$ von 0.83 bestimmt werden, daher ist für diesen wichtigen Parameter eine hohe Genauigkeit gegeben. Der Fehler der Landbedeckungsklassifikation konnte im Rahmen dieser Arbeit nicht bestimmt werden, ergibt sich aus den Angaben zum globalen Produkt allerdings zu 31\%. Fehlklassifikationen führen wahrscheinlich zu den größten Fehlern in den Modellergebnissen.

Das Modell reagiert auf Variationen der PAR ebenfalls äußerst sensibel. Daher ist es wichtig festzustellen, dass für den Parameter eine hohe Genauigkeit mit einer Korrelation von 0.79 mit gemessenen Werten bestimmt wurde. Gleiches gilt für die maximale und die minimale Temperatur, mit einem $r$ von 0.91 bzw. 0.89. Für den Niederschlag muss festgehal- 
ten werden, dass die Genauigkeit der täglichen Daten unerheblich ist, solange der mittelfristige Eintrag (Wochen bis wenige Monate) korrekt ist. Für die Monatssummen konnte eine Korrelation von nur 0.59 bestimmt werden, welcher nach den Ergebnissen der Sensitivitätsstudie allerdings nur in sehr trockenen Gebieten problematisch ist. Die schlechte Güte der Windgeschwindigkeit $(r=0.47)$ hat nur sehr geringen Einfluss auf das Modellergebnis, da das Modell nahezu gar nicht sensibel gegenüber Variationen der Windgeschwindigkeit ist.

Neben den Anpassungen des Modells an die regionalen Besonderheiten des Arbeitsgebietes, war ein weiteres Ziel dieser Arbeit, die Grundlagen für eine künftige Verwendung von BETHY/DLR als Szenariomodell zu bereiten. Im Rahmen nachfolgender Projekte ist geplant, mit Hilfe von einschlägigen Klimaszenarien und Annahmen über die Bewirtschaftungspraktiken die künftige Entwicklung der Vegetation nicht nur in Trockengebieten, sondern beispielsweise auch in Europa für die Bestimmung von potenziellen Bioenergieressourcen, zu simulieren. Erhältliche Klimaszenarien enthalten allerdings nicht die potenzielle Entwicklung aller relevanten Eingangsgrößen des Modells BETHY/DLR. Zwar können Trends von Temperatur und Niederschlag verwendet werden, angenommene Zeitreihen über die saisonale Entwicklung der Vegetation sind dagegen nicht verfügbar. Daher wurde in dieser Arbeit ein Modul implementiert, das aus den Informationen der Temperatur, der Niederschlagsmenge und der die Erdoberfläche erreichenden PAR modellintern in BETHY/DLR die Entwicklung des LAI berechnet. In diesem Phänologiemodell werden Unterschiede für die verschiedenen Vegetationstypen im Modell berücksichtigt. Das Modul beruht auf der Parametrisierung des Beginns der vegetativen Phase über eine exponentiell gewichtete Funktion der Temperatur über einen bestimmten Zeitraum. Zusätzlich wird das Ende der vegetativen Phase über die Abnahme der einfallenden Strahlung gesteuert. Außerdem erfährt die Beschreibung des LAI eine Limitierung über das pflanzenverfügbare Bodenwasser, die zusätzlich sowohl Anfang als auch Ende der produktiven Phase beeinflusst.

Für den Test dieses ersten wichtigen Bausteins auf dem Weg zur Szenariomodellierung wurde eine einfache Kalibrierung der Parameter des Moduls durchgeführt. Dabei wurden die jeweiligen Ergebnisse des Phänologiemodells bei veränderten Parametern mit den Zeitreihen des SPOT-VGT-LAI verglichen. Es wurde deutlich, dass durch das Modell die saisonale Entwicklung des LAI durchaus wiedergegeben wird. Eine Ausnahme bildet die immergrüne Vegetation, die allerdings in den Daten des SPOT-VGT-LAI meist ebenfalls mit einer hohen saisonalen Variabilität dargestellt wird, was für diesen Vegetationstyp sehr ungewöhnlich ist. Daher werden die Parameter des Modells so angepasst, dass die Variationen für immergrüne Vegetationstypen gering ausfallen. Folglich ergeben sich auch bei 
einem Vergleich mit den SPOT-VGT-Daten hohe Unterschiede. Als wichtiger Faktor für die Modellierung des LAI wird die Amplitude der saisonalen Schwingungen betrachtet. Diese wird durch das Phänologiemodell in der Regel gut wiedergegeben. Für die verschiedenen Vegetationstypen ergeben sich für einzelne Jahre Abweichungen des bestimmten maximalen LAI, meist mit einer Unterschätzung. Dabei handelt es sich meist auch in den SPOT-VGT-Zeitreihen um vergleichsweise hohe bzw. niedrige Jahreswerte bezüglich der übrigen Jahre.

Solche Abweichungen zeigen die Notwendigkeit weiterer Schritte zur Annäherung der simulierten LAI-Daten an die verfügbaren Beobachtungen. Daher wird das Modell in an diese Arbeit anknüpfenden Projekten um ein Verfahren der Assimilation von Beobachtungsdaten, wie dem SPOT-VGT-LAI oder dem MODIS-LAI, erweitert. Dieses wird die zeitintensive Kalibrierung der Modellparameter ersetzen und zu einer Verbesserung der prozessierten LAI-Zeitreihen führen.

Im Hinblick auf die Verwendung der Modellergebnisse von BETHY/DLR (GPP und NPP) zur Bewertung der Landdegradation in Trockengebieten wurden diese für das Arbeitsgebiet auf deren Genauigkeit überprüft. Für einen Vergleich wurden mehrere unterschiedliche Datensätze verwendet. Zunächst wurde die akkumulierte Produktivität der Vegetation, also die Summe der NPP über eine Vegetationsperiode mit Feldmessungen der stehenden Biomasse, verglichen. Solche Daten standen für den Krüger-Nationalpark in Südafrika für einen Zeitraum von acht Jahren zur Verfügung. In Anbetracht der Tatsache, dass die erhobenen Daten größtenteils in Gebieten durchgeführt wurden, die regelmäßig von Feuerereignissen betroffen sind, welche in der Modellformulierung nicht berücksichtigt werden, konnte dennoch ein Korrelationskoeffizient von $\mathrm{r}=0.6$ zwischen den Datensätzen ermittelt werden. Die Nichtberücksichtigung der durch die Feuerereignisse reduzierten zugewachsenen Biomasse spiegelt sich in einer Überschätzung der saisonalen Produktivität durch BETHY/DLR wieder.

Einen weiteren Datensatz für die Bewertung der Modellergebnisse stellt das Netzwerk der Eddy-Kovarianz-Türme dar, in das auch vier Messstationen im Arbeitsgebiet integriert sind. Aus Messungen des Kohlenstoffaustausches zwischen der Atmosphäre und der Vegetation wird die GEP bestimmt, die sich mit gewissen Einschränkungen mit der modellierten GPP vergleichen lässt. Auch hier ergibt sich das Problem, dass bei den Messungen Einflüsse berücksichtigt werden, die durch das Modell nicht erfasst werden können. Daher fällt der maximale Fehler aus den Vergleichen mit längeren Messreihen der Türme von etwa $50 \%$ noch relativ gering aus. Außerdem gelten für die Messungen der Stationen selbst hohe Unsicherheiten. 
Letztlich wurden die Modellergebnisse auch mit anderen Ansätzen der GPP- und NPPModellierung verglichen. Verfügbar waren globale Datensätze der NPP für 1999 und 2000 aus dem Modell und GPP- sowie NPP-Produkte, erstellt aus den Satellitendaten von MODIS für den Zeitraum 2000 bis 2010. Aus beiden Vergleichen ergeben sich sehr gute Korrelationen von 0.8 bis 0.9 , bei relativen Abweichungen von bis zu 77\%. Außerdem werden die beiden Größen durch BETHY/DLR gegenüber GloPEM und MODIS deutlich unterschätzt. Aus anderen Studien zur Bewertung der Qualität der beiden globalen Produkte lässt sich eine entsprechende Abweichung gegenüber Bodenmessungen für einzelne Regionen ablesen.

Schließlich wird für das Arbeitsgebiet des südlichen Afrikas ein mittlerer Fehler von bis zu $70 \%$ angenommen. Dieser schlägt sich auch auf die weiteren, aus der modellierten NPP abgeleiteten Produkte, nieder. Die guten Korrelationen mit den verglichenen Datensätzen bestätigen allerdings die Verwertbarkeit der relativen Trends der Pflanzenproduktivität, unabhängig von absoluten Abweichungen. Diese werden schließlich für die Bewertung der Vegetationsentwicklung im südlichen Afrika verwendet. Als Hauptthema der vorliegenden Arbeit stand die Entwicklung eines neuen Ansatzes zur Bewertung der Landdegradation im ariden und semi-ariden südlichen Afrika im Fokus. Dieses Ziel konnte mit dem vorgestellten Degradationsindex erreicht werden. Anhand der modellierten Zeitreihen der NPP der Vegetation im Arbeitsgebiet wurden zunächst Trends bestimmt, die eine Zunahme oder Reduzierung der Produktivität relativ zu dem jeweiligen Anfangswert unterscheiden. Dazu wurde der Zeitraum von 1999 bis 2010 betrachtet und die saisonalen Zuwachsraten entsprechend der jahreszeitlichen Verteilung in der südlichen Hemisphäre von Juli bis Juni des Folgejahres aufsummiert. Als Anfangswert diente demnach die Produktivität in der Vegetationsperiode 99/00. Aus diesen Zeitreihen wurde schließlich der Trend der Entwicklung bestimmt. Für die Erstellung des Degradationsindexes wurden Werte eines negativen Trends in vier Klassen eingeteilt: Dabei handelt es sich um die Reduzierung um 0 bis 25\% (Klasse 1), 25 bis 50\% (Klasse 2), 50 bis 75\% (Klasse 3) und > 75\% (Klasse 4). Dadurch kann zunächst der Grad der Degradation eingestuft werden.

Eine zusätzliche Unterteilung erfolgt durch die Betrachtung der Trends der mittleren Jahrestemperatur und der jährlichen Niederschlagssumme. Erfährt eine Region einen negativen Niederschlagstrend mit einem Rückgang der Regenmengen um mehr als 30\%, erfolgt eine Einordung in Kategorie B. Eine Region mit positivem Temperaturtrend mit einer Zunahme der Temperatur um mehr als 10\% wird die Kategorie C zugewiesen. Treffen beide Kriterien zu, erhält die entsprechende Region die Zuordnung zur Kategorie D. Kategorie A enthält die Regionen, für die keine signifikanten Trends von Niederschlagsmenge und Temperatur ermittelt wurden. Anhand dieser Unterscheidung erfolgt eine Bewertung, ob das von 
Landdegradation betroffene Gebiet alleine durch die Entwicklung der natürlichen Einflüsse belastet wird oder bei sonst guten klimatischen Bedingungen alleine durch die Beanspruchung durch den Menschen einen Rückgang an Produktivität erleidet.

Regionale Studien bestätigen mehrheitlich den bestimmten Degradationsindex. Auch die Höhe des Degradationsindexes korreliert gut mit den publizierten Graden der Degradation. Dabei konnten zahlreiche Gebiete in Namibia, Botswana, Simbabwe, Südafrika und Lesotho verglichen werden. Auch wenn eine großflächige Validierung des entwickelten Degradationsindexes aufgrund fehlender Vergleichsstudien nicht möglich ist, werden die regionalen Übereinstimmungen als Hinweis für die Anwendbarkeit der vorgestellten Methode gewertet. Damit konnte in dieser Arbeit ein erster Ansatz zur Langzeitbewertung von Degradationsprozessen auf nationaler bis subkontinentaler Skala mit hoher Auflösung entwickelt werden. Der größte Vorteil der vorgestellten Methode liegt in der Verwendung von fernerkundungsbasierten Modellergebnissen der pflanzlichen Vegetation, da diese über lange Zeiträume und für immer höher werdende Auflösungen verfügbar sind. In späteren Arbeiten sollen daher alternative Datensätze und Methoden zur möglichen Interpolation der Eingangsdaten auf die Modellauflösung getestet werden, die zu einer Qualitätsverbesserung der Parameter führen sollen.

Das vorgestellte Produkt kann der Politik als Grundlage dienen bei der Entscheidung, in welchen Regionen dringender Handlungsbedarf besteht. Auch für den privaten oder kommerziellen Sektor ist die Information über die Entwicklung der vegetativen Produktivität für die Einschätzung des Zustandes des eigenen Landes relevant. Die weitere Entwicklung des zugrunde liegenden Modells BETHY/DLR zu einem Szenariomodell erweitert die Anwendung des Ansatzes auf die Prognose der künftigen Vegetationsentwicklung unter bestimmten Annahmen zu Klimaveränderungen oder unterschiedlichen Anbaupraktiken. Auch die unzureichende Datengrundlage zur Validierung der Modellergebnisse von BETHY/DLR oder der Bewertung des Degradationszustandes selbst wird Bestandteil weiterer Studien, auch in anderen ariden Gebieten der Erde, sein. Diese Entwicklungen werden weiter dazu beitragen, den vorgestellten Ansatz noch verlässlicher und genauer zu machen. 


\section{Anhang}

\section{Anhang I. Vergleich der MODIS-GPP- und NPP-Produkte}
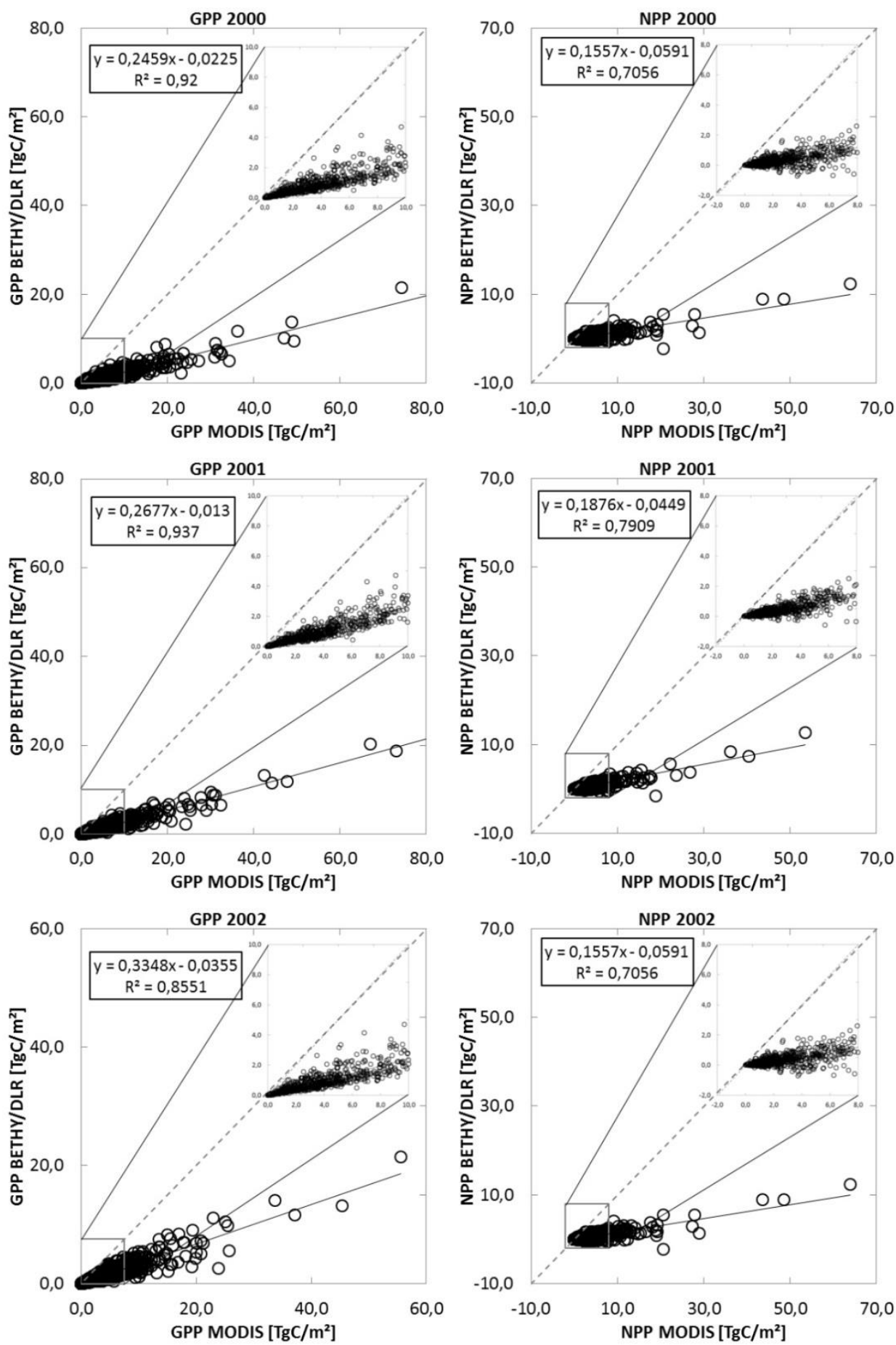

Abbildung A.1: Vergleich der Modellergebnisse aus BETHY/DLR und MODIS für die GPP (links) und die NPP (rechts) für die Jahre 2000 (oben), 2001 (Mitte) und 2002 (unten). Die gestrichelte Linie stellt die 1:1-Linie dar. Innerhalb der Abbildungen ist der Bereich kleinerer Werte vergrößert dargestellt. 

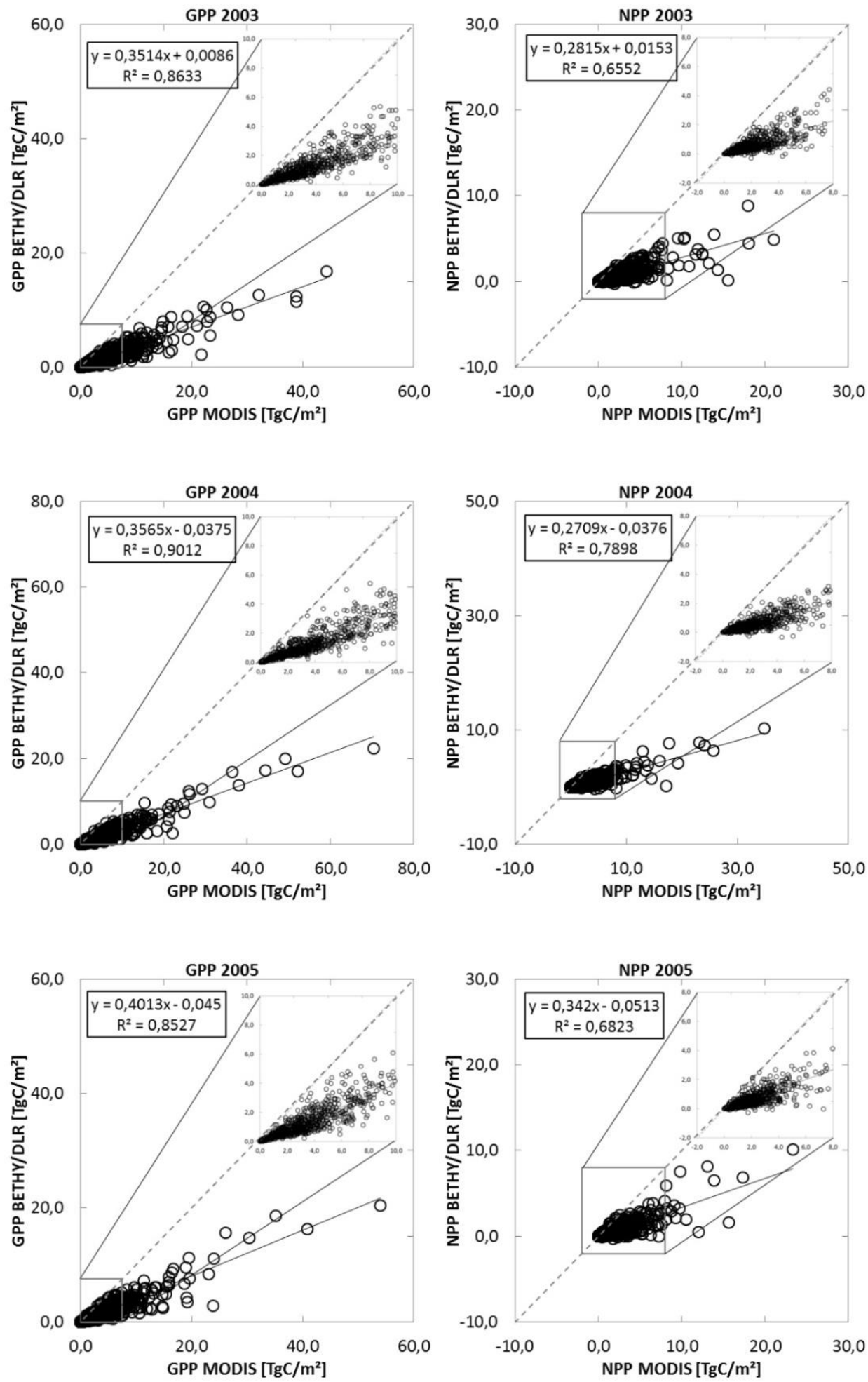

Abbildung A.2: Vergleich der Modellergebnisse aus BETHY/DLR und MODIS für die GPP (links) und die NPP (rechts) für die Jahre 2003 (oben), 2004 (Mitte) und 2005 (unten). Die gestrichelte Linie stellt die 1:1-Linie dar. Innerhalb der Abbildungen ist der Bereich kleinerer Werte vergrößert dargestellt. 

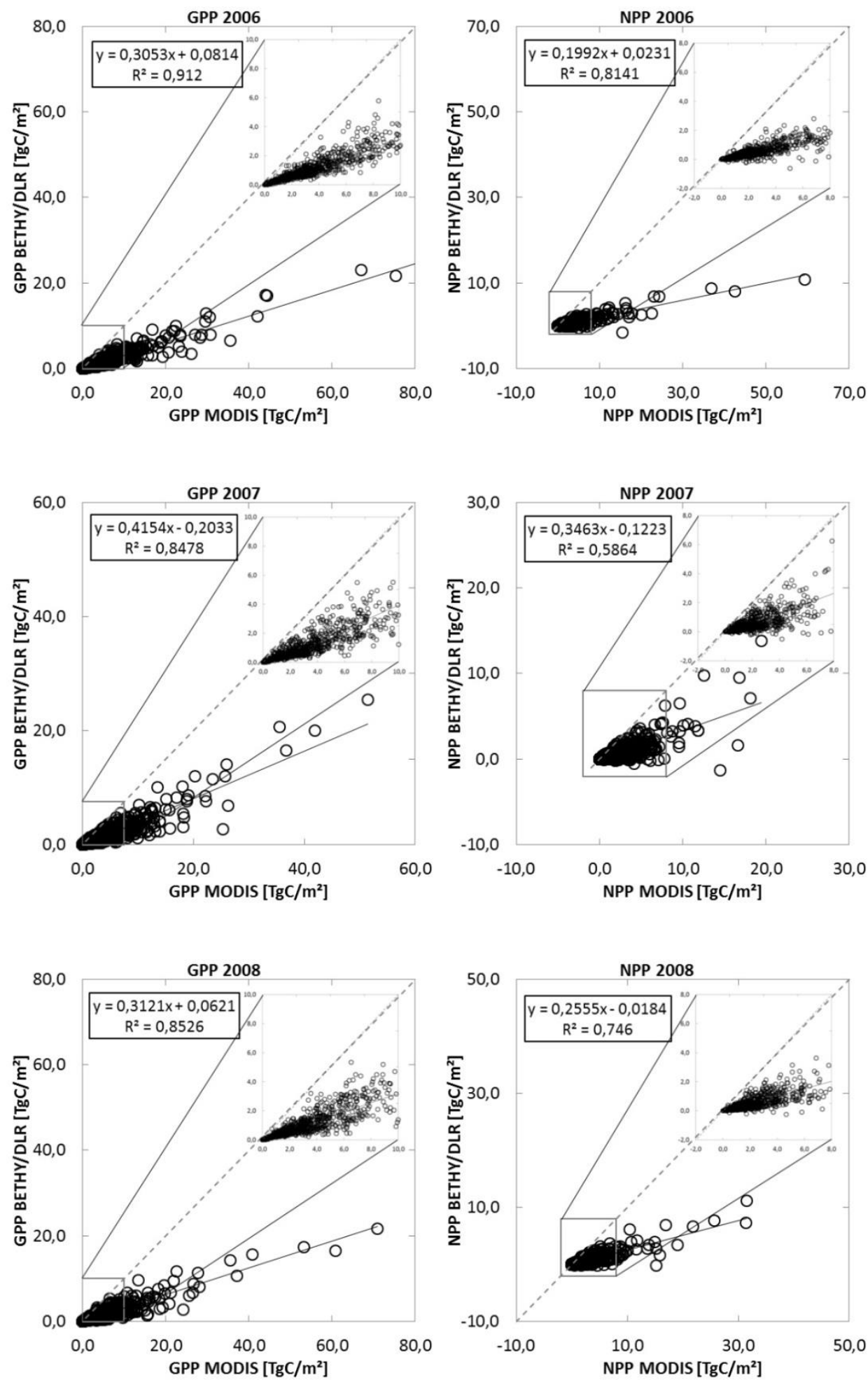

Abbildung A.3: Vergleich der Modellergebnisse aus BETHY/DLR und MODIS für die GPP (links) und die NPP (rechts) für die Jahre 2006 (oben), 2007 (Mitte) und 2008 (unten). Die gestrichelte Linie stellt die 1:1-Linie dar. Innerhalb der Abbildungen ist der Bereich kleinerer Werte vergrößert dargestellt. 

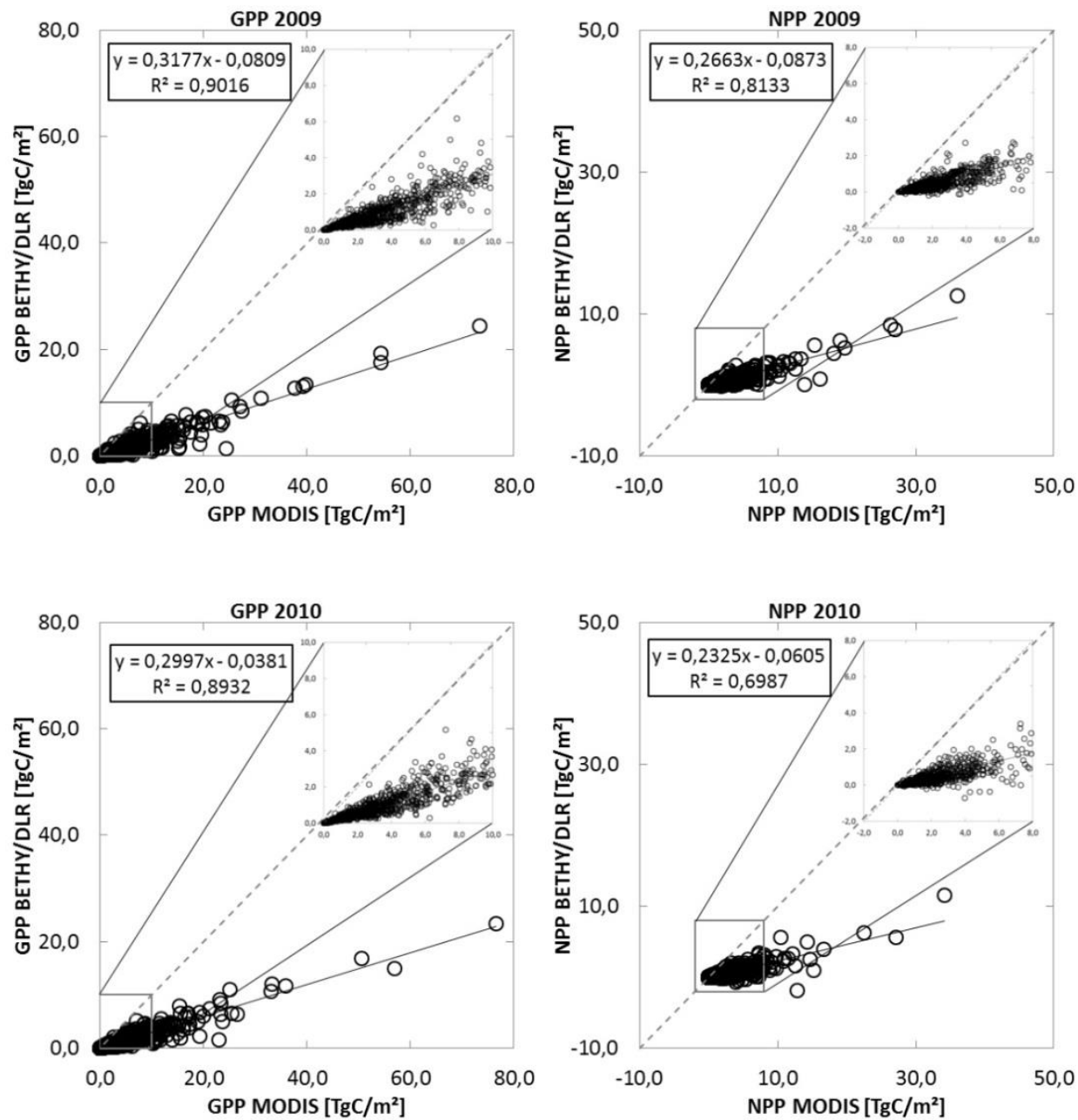

Abbildung A.4: Vergleich der Modellergebnisse aus BETHY/DLR und MODIS für die GPP (links) und die NPP (rechts) für die Jahre 2009 (oben) und 2010 (unten). Die gestrichelte Linie stellt die 1:1-Linie dar. Innerhalb der Abbildungen ist der Bereich kleinerer Werte vergrößert dargestellt. 


\section{Literaturverzeichnis}

Abel, N. O. J. and Blaikie, P. M. (1989). Land degradation, stocking rates and conservation policies in the communal rangelands of Botswana and Zimbabwe. Land Degradation and Rehabilitation, 1(2):101-123.

Al-Awadhi, J. M. (2011). A case assessment of the mechanisms involved in human-induced land degradation in northeastern Kuwait. Land Degradation \& Development.

Baldocchi, D. D., Hincks, B. B., and Meyers, T. P. (1988). Measuring biosphereatmosphere exchanges of biologically related gases with micrometeorological methods. Ecology, 69(5):1331-1340.

Baret, F., Hagolle, O., Geiger, B., Bicheron, P., Miras, B., Huc, M., Berthelot, B., Nino, F., Weiss, M., Samain, O., Roujean, J. L., and Leroy, M. (2007). Lai, fapar and fcover cyclopes global products derived from vegetation: Part 1: Principles of the algorithm. Remote Sensing of Environment, 110(3):275 - 286.

Baret, F., Makhmara, H., Lacaze, R., and Smets, B. (2010). BioPar Product User Manual LAI, FAPAR, FCover, NDVI Version 1 from SPOT/VEGETATION data. INRA.

Bartholome, E., Belward, A., Achard, F., Bartalev, S., Carmonamoreno, C., Eva, H., Fritz, S., Gregoire, J.-M., Mayaux, P., and Stibig, H.-J. (2002). GLC2000; Global land cover mapping for the year 2000. Project status November 2002. Technical report, Institute for Environment and Sustainability, Joint Research Centre, ISPRA, Italy.

Bartholomé, E. and Belward, A. S. (2005). GLC2000: A new approach to global land cover mapping from Earth Observation data. International Journal of Remote Sensing, 26:1959-1977.

Beerling, D. and Quick, W. (1995). A new technique for estimating rates of carboxylation and electron transport in leaves of $\mathrm{c} 3$ plants for use in dynamic global vegetation models. Global Change Biology, 1(4):289-294.

Bittner, M., Dech, S., and Balzer, W. (1998). Ozonkartierung per Satellit. Spektrum der Wissenschaft, 9:54-61. 
Bittner, M., Dech, S. W., and Loyola, D. (1997). Planetary-scale waves in total ozone from ERS-2 GOME data. In T.-D. Guyenne \& D. Danesy, editor, Third ERS Symposium on Space at the service of our Environment, Volume 414 of ESA Special Publication, pages 703 .

Bittner, M., Offermann, D., Bugaeva, I., Kokin, G., Koshelkov, J., Krivolutsky, A., Tarasenko, D., Gil-Ojeda, M., Hauchecorne, A., Lübken, F.-J., de la Morena, B., Mourier, A., Nakane, H., Oyama, K., Schmidlin, F., Soule, I., Thomas, L., and Tsuda, T. (1994). Long period/large scale oscillations of temperature during the dyana campaign. Journal of Atmospheric and Terrestrial Physics, 56(13-14):1675 - 1700. Dynamic Adapted Network for the the Atmosphere.

Bolz, H. M. (1949). Die Abhängigkeit der infraroten Gegenstrahlung von der Bewölkung. Zeitschrift für Meteorologie, 7:201-203.

Bosch, O., van Rensburg, F. J., and du T. Truter, S. (1987). Identification and selection of benchmark sites on Litholitic soils of the western grassland biome of South Africa. Journal of the Grassland Society of Southern Africa, 4(2):59-62.

Bosch, O. and vanRensburg, F. J. (1987). Ecological status of species on grazing gradients on the shallow soils of the western grassland biome in South Africa. Journal of the Grassland Society of Southern Africa, 4(4):143-147.

Botta, A., Viovy, N., Ciais, P., Friedlingstein, P., and Monfray, P. (2000). A global prognostic scheme of leaf onset using satellite data. Global Change Biology, 6(7):709-725.

Brutsaert, W. (1982). Evaporation into the atmosphere. Reidel Publishing, Dordrecht, Netherlands.

Budyko, M. L. (1969). The effect of solar radiation variations on the climate of the earth. Tellus, 21:611-619.

Burridge, D. M. and Gadd, A. J. (1974). The Meteorological Office Operational 10 Level Numerical Weather Prediction Model. Technical report, British Met. Office Tech. Notes Nos. 12 and 48, England.

Businger, J. A. (1986). Evaluation of the Accuracy with Which Dry Deposition Can Be Measured with Current Micrometeorological Techniques. Journal of Applied Meteorology, 25:1100-1124. 
Campbell, B. (1996). The Miombo in Transition: Woodlands and Welfare in Africa. Center for International Forestry Research, Bogor, Indonesia.

CGER (2000). Ecological Indicators for the Nation. The National Academies Press.

Cholesky, A.-L. (1924). Note sur une méthode de résolution des equations normales provenant de l'application de la méthode des moindres carrés á un systéme d'équations linéaires en nombre inférieur á celui des inconnues (Procédé du Commandant Cholesky). Bulletin Géodésique, 2:67-77.

Collatz, G., Ball, J., Grivet, C., and Berry, J. A. (1991). Physiological and environmental regulation of stomatal conductance, photosynthesis and transpiration: a model that includes a laminar boundary layer. Agricultural and Forest Meteorology, 54:107 136.

Collatz, G. J., Ribas-Carbo, M., and Berry, J. A. (1992). Coupled photosynthesis-stomatal conductance model for leaves of C4 plants. Australian Journal of Plant Physiology, 19(5):519-538.

Cowling, R. M., Richardson, D. M., and Pierce, S. M. (1997). Vegetation of Southern Africa. Cambirdge University Press.

Darkoh, M. B. K. (1999). Case studies of rangeland desertification, chapter Desertification in Botswana, pages 61-74. Agricultural Research Institute, Rekjavik.

De Queiroz, J. S. (1993). Range degradation in botswana myth or reality? Technical report, Pastoral Development Network. Overseas Development Inst., London (United Kingdom).

Di Gregorio, A. D. and Jansen, L. J. (1998). Land Cover Classification System (LCCS): Classification Concepts and User Manual. Food and Agriculture Organization of the United Nations.

DKRZ (1993). The ECHAM3 Atmospheric General Circulation Model. Technical report, Deutsches Klimarechenzentrum (DKRZ) Modellbetreuungsgruppe.

Dougill, A. J. and Cox, J. (1995). Land degradation and grazing in the Kalahari: New analysis and alternative perspectives. ODI Pastoral Development Network Paper, 38. 
Dougill, A. J., Thomas, D. S. G., and Heathwaite, A. L. (1999). Environmental Change in the Kalahari: Integrated Land Degradation Studies for Nonequilibrium Dryland Environments. Annals of the Association of American Geographers, 89(3):420-442.

Dufresne, J.-L., Friedlingstein, P., Berthelot, M., Bopp, L., Ciais, P., Fairhead, L., Le Treut, H., and Monfray, P. (2002). On the magnitude of positive feedback between future climate change and the carbon cycle. Geophysical Research Letters, 29:1405-1408.

Erbertseder, T., Eyring, V., Bittner, M., Dameris, M., and Grewe, V. (2006). Hemispheric ozone variability indices derived from satellite observations and comparison to a coupled chemistry-climate model. Atmospheric Chemistry and Physics, 6:51055120.

Etheridge, D., Steele, L., Langenfelds, R., and Francey, R. (1996). Natural and anthropogenicc hangesin atmospheric $\mathrm{CO} 2$ over the last 1000 years from air in Antarctic ice and firn. Journal of Geophysical Research, 101:4115-4128.

Evans, J. and Geerken, R. (2004). Discrimination between climate and human-induced dryland degradation. Journal of Arid Environments, 57:535-554.

Falge, E., Baldocchi, D., Olson, R., Anthoni, P., Aubinet, M., Bernhofer, C., Burba, G., Ceulemans, R., Clement, R., Dolman, H., Granier, A., Gross, P., Grünwald, T., Hollinger, D., Jensen, N.-O., Katul, G., Keronen, P., Kowalski, A., Lai, C. T., Law, B. E., Meyers, T., Moncrieff, J., Moors, E., Munger, J., Pilegaard, K., Ãllar Rannik, Rebmann, C., Suyker, A., Tenhunen, J., Tu, K., Verma, S., Vesala, T., Wilson, K., and Wofsy, S. (2001). Gap filling strategies for defensible annual sums of net ecosystem exchange. Agricultural and Forest Meteorology, 107(1):43 - 69.

FAO, IIASA, ISRIC, ISSCAS and JRC (2009). Harmonized World Soil Database (version 1.1). FAO, Rome, Italy and IIASA, Laxenburg, Austria.

Farquhar, G. D., Caemmerer, S., and Berry, J. A. (1980). A biochemical model of photosynthetic CO2 assimilation in leaves of C3 species. Planta, 149:78-90. 10.1007/BF00386231.

Farquhar, G. D. and Sharkey, T. D. (1982). Stomatal conductance and photosynthesis. Annual Review of Plant Physiology, 33:317-345.

Federer, C. A. (1979). A Soil-Plant-Atmosphere Model for Transpiration and Availability of Soil Water. Water Resources Research, 15:555-562. 
Federer, C. A. (1982). Transpirational Supply and Demand: Plant, Soil, and Atmospheric Effects Evaluated by Simulation. Water Resources Research, 18:355-362.

Foley, J. A., Levis, S., Prentice, I. C., and Pollard, D. (1998). Coupling dynamic models of climate and vegetation. Global Change Biology, 4:561-579.

Fox, R. and Rowntree, K. (2001). Redistribution, restitution and reform: Prospects for the land in the Eastern Cape Province, South Africa. In: A. Conacher (Ed.), Land degradation (pp. 167-186). London: Kluwer Academic Publishing.

Fritz, S., Bartholome, E., Belward, A., Hartley, A., Stibig, H.-J., Eva, H., Mayaux, P., Bartalev, S., Latifovic, R., Kolmert, S., Sarathi, R., Aggarwal, S., Bingfang, W.,Wenting, X., Ledwith, M., Pekel, J., Giri, C., Mucher, S., De Badts, E., Tateishi, R., Champeaux, J., and Defourny, P. (2003). Harmonisation, mosaicing and production of the global land cover 2000 database (beta version). Technical report, EUR 20849 EN, European Commission, Directorate General, Joint Research Centre (JRC).

Garrigues, S., Lacaze, R., Baret, F., Morisette, J. T., Weiss, M., Nickeson, J. E., Fernandes, R., Plummer, S., Shabanov, N. V., Myneni, R. B., Knyazikhin, Y., and Yang, W. (2008). Validation and intercomparison of global Leaf Area Index products derived from remote sensing data. Journal of Geophysical Research (Biogeosciences), 113(12):G02028.

GCOS (2003). The second report on adequacy of global observation systems for climate in support of the unfccc - executive summary. Technical report, World Meteorological Organization.

Geiger, R. (1954). Landolt-Börnstein - Zahlenwerte und Funktionen aus Physik, Chemie, Astronomie, Geophysik und Technik, alte Serie Vol. 3, Chapter: Klassifikation der Klimate nach W. Köppen, pages 603-607. Springer, Berlin.

Goetz, S. J., Prince, S. D., Goward, S. N., Thawley, M. M., Small, J., and Johnston, A. (1999). Mapping net primary production and related biophysical variables with remote sensing: Application to the boreas region. Journal of Geophysical Research, 104(D22):27719-27734. 
GTOS, G. T. O. S. (2009). Biomass. Assessment of the status of the development of the standards for the terrestrial essential climate variables. Technical report, Food and Agriculture Organization of the United Nations (FAO).

Hamandawana, H., Chanda, R., and Eckardt, F. (2007). Natural and humaninduced environmental changes in the semi-arid distal reaches of Botswana's Okavango delta. Journal of Land Use Science, 2:57-78.

Hamandawana, H., Eckardt, F., and Chanda, R. (2005). Linking archival and remotely sensed data for long-term environmental monitoring. International Journal of Applied Earth Observation and Geoinformation, 7(4):284 - 298.

Hannerz, M. (1999). Evaluation of temperature models for predicting bud burst in norway spruce. Canadian Journal of Forest Research, 29(1):9-19.

Hänninen, H. (1995). Effects of climatic change on trees from cool and temperate regions: an ecophysiological approach to modelling of bud burst phenology. Canadian Journal of Botany, 73:183-199.

Haxeltine, A. and Prentice, I. (1996). BIOME3: An Equilibrium Terrestrial Biosphere Model Based on Ecophysiological Constraints, Resource Availability, and Competition Among Plant Functional Types. Global Biogeochemical Cycles, 10(4):693-709.

Heide, O. M. (1993a). Daylength and thermal time responses of budburst during dormancy release in some northern deciduous trees. Physiologia Plantarum, 88(4):531-540.

Heide, O. M. (1993b). Dormancy release in beech buds (fagus sylvatica) requires both chilling and long days. Physiologia Plantarum, 89(1):187-191.

Heinsch, F. A., Reeves, M., Votava, P., Kang, S., Milesi, C., Zhao, M., Glassy, J., Jolly, W., Loehman, R., Bowker, C., Kimball, J., Nemani, R. R., and Running, S. W. (2003). User's guide: GPP and NPP (MOD17A2/A3) products, NASA MODIS land algorithm, Version 2.0. Missoula, MT: Univ. Montana.

Heinsch, F. A., Zhao, M., Running, S. W., Kimball, J. S., Nemani, R. R., Davis, K. J., Bolstad, P. V., Cook, B. D., Desai, A. R., Ricciuto, D. M., Law, B. E., Oechel, W. C., Kwon, H., Luo, H., Wofsy, S. C., Dunn, A. L., Munger, J. W., Baldocchi, D. D., Xu, L., Hollinger, D. Y., Richardson, A. D., Stoy, P. C., Siqueira, M. B. S., Monson, R. K., Burns, S. P., and Flanagan, L. B. (2006). Evaluation of Remote Sensing Based Terrestrial Productivity From MODIS Using Regional Tower Eddy Flux Network 
Observations. IEEE Transactions on Geoscience and Remote Sensing, 44:19081925.

Hoffman, M. and Todd, S. (2000). A National Review of Land Degradation in South Africa: the Influence of Biophysical and Socio-economic Factors. Journal of Southern African Studies, 26:743-758.

Hoffman, T., Todd, S., Ntoshona, Z., and S., T. (1999). Land degradation in south africa. Technical report, Cape Town National Botanical Institute.

Hunter, A. F. and Lechowicz, M. J. (1992). Predicting the timing of budburst in temperate trees. Journal of Applied Ecology, 29:597-604.

IPCC (2001). Climate Change 2001: Impacts, Adaptation, and Vulnerability. Cambirdge University Press, New York.

IPCC (2007a). Climate Change 2007: Mitigation of Climate Change. Contribution of Working Group III to the Fourth Assessment Report of the Intergovernmental Panel on Climate Change. IPCC.

IPCC (2007b). Climate Change 2007: Synthesis Report. Contribution of Working Groups I, II and III to the Fourth Assessment Report of the Intergovernmental Panel on Climate Change, volume 446. IPCC.

Jiao, Y., Xu, Z., and Zhao, J. (2009). Effects of grassland conversion to cropland and forest on soil organic carbon and dissolved organic carbon in the farming-pastoral ecotone of inner mongolia. Acta Ecologica Sinica, 29(3):150 - 154.

Jones, H. G. (1983). Plants and Microclimate. Cambridge University Press, Cambridge.

Kaduk, J. and Heimann, M. (1996). A prognostic phenology scheme for global terrestrial carbon cycle models. Climate Research, 6:1-19.

Keeling, C. D. (1960). The concentration and isotopic abundances of carbon dioxide in the atmosphere. Tellus, 12(2):200-203.

Kelliher, F. M., Leuning, R., and Schulze, E. D. (1993). Evaporation and canopy characteristics of coniferous forests and grasslands. Oecologia, 95:153-163. 10.1007/BF00323485. 
Knorr, W. (1997). Satellite Remote Sensing and Modelling of the Global COExchange of Land Vegetation: A Synthesis Study. PhD thesis, Faculty ofEarth Sciences of the University of Hamburg, Hamburg.

Knorr, W. and Heimann, M. (2001a). Uncertainties in global terrestrial biosphere modeling, Part I: A comprehensive sensitivity analysis with a new photosynthesis and energy balance scheme. Global Biogeochemical Cycles, 15(1):207-225.

Knorr, W. and Heimann, M. (2001b). Uncertainties in global terrestrial biosphere modeling, Part II: Global constraints for a process-based vegetation model. Global Biogeochemical Cycles, 15(1):227-246.

Knorr, W., Kaminski, T., Scholze, M., Gobron, N., Pinty, B., Giering, R., and Mathieu, P.-P. (2010). Carbon cycle data assimilation with a generic phenology model. Journal of Geophysical Research, 115.

Köppen, W. (1900). Versuch einer Klassifikation der Klimate, vorzugsweise nach ihren Beziehungen zur Pflanzenwelt. Geographische Zeitschrift, 12:657-679.

Kottek, M., Grieser, J., Beck, C., Rudolf, B., and Rubel, F. (2006). World Map of the Köppen-Geiger climate classification updated. Meteorologische Zeitschrift, 15(3):259263.

Krinner, G., Viovy, N., de Noblet-Ducoudré, N., Ogée, J., Polcher, J., Friedlingstein, P., Ciais, P., Sitch, S., and Prentice, I. C. (2005). A Dynamic Global Vegetation Model for Studies of the Coupled Atmosphere-Biosphere System. Global Biogeochemical Cycles, 19(GB1015).

Kurihara, Y. (1970). A Statistical-Dynamical Model of the General Circulation of the Atmosphere. Journal of Atmospheric Sciences, 27:847-870.

Landmann, T. (2003). A case study for Skukuza: Estimating biophysical properties of fires using EOS-MODIS satellite data. PhD thesis, Georg-August-Universität zu Göttingen.

Law, B., Thornton, P., Irvine, J., Anthoni, P., and Van Tuyl, S. (2001). Carbon storage and fluxes in ponderosa pine forests at different developmental stages. Global Change Biology, 7(7):755-777. 
Le Houérou, H. N. (1996). Climate change, drought and desertification. Journal of Arid Environments, 34(2):133 - 185.

Leemans, R. and Cramer, W. (1991). The IIASA database for mean monthly values of temperature, precipitation, and cloudiness on a global terrestrial grid. Research report rr91-18 November 1991, International Institute of Applied Systems Analyses: Laxenburg, Austria.

Lloyd, J. and Taylor, J. A. (1994). On the Temperature Dependence of Soil Respiration. Functional Ecology, 8(3):315-323.

Majara, N. (2005). Land degradation in Lesotho: a synoptic perspective. $\mathrm{PhD}$ thesis, University of Stellenbosch.

Mambo, J. and Archer, E. (2007). An assessment of land degradation in the Save catchment of Zimbabwe. Area, 39(3):380-391.

Manabe, S. and Möller, F. (1961). On the Radiative Equilibrium and Heat Balance of the Atmosphere. Monthly Weather Review, 89:503.

Manabe, S. and Strickler, R. F. (1964). Thermal Equilibrium of the Atmosphere with a Convective Adjustment. Journal of the Atmospheric Sciences, 21:361-385.

Mayaux, P., BartholomÃc , E., Fritz, S., and Belward, A. (2004). A new landcover map of africa for the year 2000. Journal of Biogeography, 31(6):861-877.

Mayaux, P., Eva, H., Gallego, J., Strahler, A., Herold, M., Agrawal, S., Naumov, S., De Miranda, E., Di Bella, C., Ordoyne, C., Kopin, Y., and Roy, P. (2006). Validation of the Global Land Cover 2000 Map. IEEE Transactions on Geoscience and Remote Sensing, 44(7):1728 -1739.

McGuffie, K. and Henderson-Sellers, A. (2001). Forty years of numerical climate modelling. International Journal of Climatology, 21(9):1067-1109.

Meisner, R. E., Bittner, M., and Dech, S. W. (1999). Computer animation of remote sensing-based time series data sets. IEEE Transactions on Geoscience and Remote Sensing, 37:1100-1106.

Metz, B., Davidson, O. R., Bosch, P. R., Dave, R., and Meyer, L. A. (eds). 2007. Climate Change 2007: Mitigation of Climate Change. Contribution of Working Group III to 
the Fourth Assessment Report of the Intergovernmental Panel on Climate Change. Cambridge: Cambridge University Press.

Moncrieff, J., Malhi, Y., and Leuning, R. (1996). The propagation of errors in long-term measurements of land-atmosphere fluxes of carbon and water. Global Change Biology, 2(3):231-240.

Monsi, M. and Saeki, T. (1953). Über den Lichtfaktor in den Pflanzengesellschaften und seine Bedeutung für die Stoffproduktion. Japanese Journal of Botany, 14(1):22-52.

Monteith, J. L. (1965). Light distribution and photosynthesis in field crops. Annals of Botany, 29(1):17-37.

Monteith, J. L. (1972). Solar radiation and productivity in tropical ecosystems. Journal of Applied Ecology, 9:747-766.

Monteith, J. L. (1977). Climate and the Efficiency of Crop Production in Britain. Royal Society of London Philosophical Transactions Series B, 281:277-294.

Morisette, J., Baret, F., Privette, J., Myneni, R., Nickeson, J., Garrigues, S., Shabanov, N., Weiss, M., Fernandes, R., Leblanc, S., Kalacska, M., Sanchez-Azofeifa, G., Chubey, M., Rivard, B., Stenberg, P., Rautiainen, M., Voipio, P., Manninen, T., Pilant, A., Lewis, T., Iiames, J., Colombo, R., Meroni, M., Busetto, L., Cohen, W., Turner, D., Warner, E., Petersen, G., Seufert, G., and Cook, R. (2006). Validation of global moderate resolution LAI products: a framework proposed within the CEOS land product validation subgroup. IEEE Transactions on Geoscience and Remote Sensing, 44(7):1804-1817.

Morison, J. I. L. (1987). Intercellular $\mathrm{CO}_{2}$ concentration and stomatal response to $\mathrm{CO}_{2}$. In: Stomatal Function. (Zeiger, E., Farquhar, G.D. und Cowan, I.R. Eds.), Stanford University Press, Stanford, U.S.A., pp. 229-251.

Murray, F. W. (1967). On the Computation of Saturation Vapor Pressure. Journal of Applied Meteorology, 6:203-204.

Murray, M. B., Cannell, M. G. R., and Smith, R. I. (1989). Date of budburst of fifteen tree species in Britain following climatic warming. Journal of Applied Ecology, 26(2):693-700. 
Myhre, G. and Myhre, A. (2003). Uncertainties in Radiative Forcing due to Surface Albedo Changes Caused by Land-Use Changes. Journal of Climate, 16:1511-1524.

Nakicenovic, N., Alcamo, J., Davis, G., de Vries, B., Fenhann, J., Gaffin, S., Gregory, K., Grubler, A., Jung, T. Y., Kram, T., La Rovere, E. L., Michaelis, L., Mori, S., Morita, T., Pepper, W., Pitcher, H. M., Price, L., Riahi, K., Roehrl, A., Rogner, H.-H., Sankovski, A., Schlesinger, M., Shukla, P., Smith, S. J., Swart, R., van Rooijen, S., Victor, N., and Dadi, Z. (2000). IPCC Special Report Emissions Scenarios. Cambridge University Press, New York, NY (US).

Ortega, J. M. and Rheinboldt, W. C. (1970). Iterative Solution of Nonlinear Equations in Several Variables. Society for Industrial and Applied Mathematics.

Paltridge, G. W. and Platt, C. M. (1976). Radiative Processes in Meteorology and Climatology. Elsevier Publishing, New York.

Pallardy, S. G. (2008). Physiology of Woody Plants. Elsevier Inc.

Perkins, J. S. and Thomas, D. S. G. (1993). Spreading deserts or spatially confined environmental impacts? Land degradation and cattle ranching in the Kalahari Desert of Botswana. Land Degradation \& Development, 4(3):179-194.

Pinker, R. T. and Laszlo, I. (1992). Global Distribution of Photosynthetically Active Radiation as Observed from Satellites. Journal of Climate, 5:56-65.

Potter, C. S., Randerson, J. T., Field, C. B., Matson, P. A., Vitousek, P. M., Mooney, H. A., and Klooster, S. A. (1993). Terrestrial ecosystem production: A process model based on global satellite and surface data. Global Biogeochemical Cycles, 7:811-841.

Prentice, I. C., Cramer, W., Harrison, S. P., Leemans, R., Monserud, R. A., and Solomon, A. M. (1992). A global biome model based on plant physiology and dominance, soil properties and climate. Journal of Biogeography, 19:117-134.

Prince, S. D. and Goward, S. N. (1995). Global primary production: A remote sensing approach. Journal of Biogeography, 22(4/5):815-835.

Prince, S. D. and Small, J. (2003). Global production efficiency model. 1999_npp_latlon; 2000_npp_latlon 
Qi, Y., Dong, Y., Peng, Q., Xiao, S., He, Y., Liu, X., Sun, L., Jia, J., and Yang, Z. (2012). Effects of a conversion from grassland to cropland on the different soil organic carbon fractions in Inner Mongolia, China. Journal of Geographical Sciences, 22:315328. 10.1007/s11442-012-0929-y.

Rannik, U., Kolari, P., Vesala, T., and Hari, P. (2006). Uncertainties in measurement and modelling of net ecosystem exchange of a forest. Agricultural and Forest Meteorology, 138(1-4):244-257.

Rayner, P. J., Scholze, M., Knorr, W., Kaminski, T., Giering, R., and Widmann, H. (2005). Two decades of terrestrial carbon fluxes from a carbon cycle data assimilation system (CCDAS). Global Biogeochemical Cycles, 19(2).

Reichstein, M., Falge, E., Baldocchi, D., Papale, D., Aubinet, M., Berbigier, P., Bernhofer, C., Buchmann, N., Gilmanov, T., Granier, A., Grünwald, T., Havránková, K., Ilvesniemi, H., Janous, D., Knohl, A., Laurila, T., Lohila, A., Loustau, D., Matteucci, G., Meyers, T., Miglietta, F., Ourcival, J.-M., Pumpanen, J., Rambal, S., Rotenberg, E., Sanz, M., Tenhunen, J., Seufert, G., Vaccari, F., Vesala, T., Yakir, D., and Valentini, R. (2005). On the separation of net ecosystem exchange into assimilation and ecosystem respiration: review and improved algorithm. Global Change Biology, 11(9):1424-1439.

Richardson, A. D., Braswell, B. H., Hollinger, D. Y., Burman, P., Davidson, E. A., Evans, R. S., Flanagan, L. B., Munger, J. W., Savage, K., Urbanski, S. P., and Wofsy, S. C. (2006). Comparing simple respiration models for eddy flux and dynamic chamber data. Agricultural and Forest Meteorology, 141:219 - 234.

Ringrose, S., Musisi-Nkambwe, S., Coleman, T., Nellis, D., and Bussing, C. (1999). Use of Landsat thematic mapper data to assess seasonal rangeland changes in the southeast Kalahari, Botswana. Environmental Management, 23:125-138. 10.1007/s002679900173.

Ritchie, J. T. (1972). Model for Predicting Evaporation from a Row Crop with Incomplete Cover. Water Resources Research, 8:1204-1213.

Rosenberg, N. J. (1974). Microclimate : the biological environment. New York : Wiley.

Ross, R. (1999). A concise history of South Africa. Cambridge University Press. Cape Town. 
Roujean, J.-L., Leroy, M., and Deschamps, P.-Y. (1992). A bidirectional reflectance model of the earth's surface for the correction of remote sensing data. Journal of Geophysical Research, 97(D18):20455-20468.

Running, S. W., Nemani, R. R., Heinsch, F. A., Zhao, M., Reeves, M., and Hashimoto, H. (2004). A Continuous Satellite-Derived Measure of Global Terrestrial Primary Production. BioScience, 54(6).

Ryan, M. G. (1991). Effects of climate change on plant respiration. Ecological Applications, 1:157-167.

Sabine, C. L., Feely, R. A., Gruber, N., Key, R. M., Lee, K., Bullister, J. L., Wanninkhof, R., Wong, C. S., Wallace, D. W., Tilbrook, B., Millero, F. J., Peng, T. H., Kozyr, A., Ono, T., and Rios, A. F. (2004). The Oceanic Sink for Anthropogenic CO2. Science, 305:367-371.

Saltzman, B. (1978). A Survey of Statistical-Dynamical Models of the Terrestrial Climate. Advances in Geophysics, 20:184.

SANBI (2009). Updating national land cover. Technical report, SANBI (South African National Biodiversity Institute), Pretoria.

Sarmiento, J. L. and Gruber, N. (2006). Ocean Biogeochemical Dynamics. Princeton University Press.

Schimel, D. S. (1995). Terrestrial ecosystems and the carbon cycle. Global Change Biology, 1(1):77-91.

Schönwiese, C. (1985). Praktische Statistik für Meteorologen und Geowissenschaftler. Stuttgart: Gebrüder Borntraeger.

Schulze, E., Kelliher, F. M., Korner, C., Lloyd, J., and Leuning, R. (1994). Relationships among Maximum Stomatal Conductance, Ecosystem Surface Conductance, Carbon Assimilation Rate, and Plant Nitrogen Nutrition: A Global Ecology Scaling Exercise. Annual Review of Ecology and Systematics, 25(1), 629-662. JSTOR.

Scoones, I. (1992). Coping with drought: Responses of herders and livestock in contrasting savanna environments in southern Zimbabwe. Human Ecology, 20:293-314. 
Sellers, P., Randall, D., Collatz, G., Berry, J., Field, C., Dazlich, D., Zhang, C., Collelo, G., and Bounoua, L. (1996). A revised land surface parameterization (SIB2) for atmospheric GCMS. Part I: Model formulation. Journal of Climate, 9(4):676-705.

Sellers, P. J. (1985). Canopy reflectance, photosynthesis and transpiration. International Journal of Remote Sensing, 6(8):1335-1372.

Sellers, W. D. (1969). A Global Climatic Model Based on the Energy Balance of the EarthAtmosphere System. Journal of Applied Meteorology, 8:392-400.

Smith, B., Prentice, I. C., and Sykes, M. T. (2001). Representation of vegetation dynamics in the modelling of terrestrial ecosystems: comparing two contrasting approaches within european climate space. Global Ecology and Biogeography, 10(6):621-637.

Stoy, P. C., Katul, G. G., Siqueira, M. B., Juang, J.-Y., Novick, K. A., Uebelherr, J. M., and Oren, R. (2006). An evaluation of models for partitioning eddy covariance-measured net ecosystem exchange into photosynthesis and respiration. Agricultural and Forest Meteorology, 141(1):2 - 18 .

Stringer, L. C. and Reed, M. S. (2007). Land degradation assessment in Southern Africa: integrating local and scientific knowledge bases. Land Degradation \& Development, 18(1):99-116.

Strohbach, B. (2001). Vegetation degradation in namibia. Journal of the Namibia Scientific Society, 49:127-156.

Strohbach, B. J. (2000a). Vegetation degradation trends in the northern Oshikoto Region: I. The Hyphaene petersiana plains. Dinteria, 26:45-62.

Strohbach, B. J. (2000b). Vegetation degradation trends in the northern Oshikoto Region: II. The Colophospermum mopane shrublands. Dinteria, 26:45-62.

Stull, R. B. (1988). An Introduction to Boundary Layer Meteorology. Kluwer Academic Publishers, Dordrecht, Netherlands.

Sundquist, E. T. and Keeling, R. F. (2009). The Mauna Loa carbon dioxide record: Lessons for long-term earth observations. Geophysical Monograph Series, 183:27-35.

Tchuenté , A. T. K., Roujean, J.-L., and Jong, S. M. D. (2011). Comparison and relative quality assessment of the glc2000, globcover, modis and ecoclimap land cover data 
sets at the african continental scale. International Journal of Applied Earth Observation and Geoinformation, 13(2):207 - 219.

Tetzlaff, G. and Peters, M. (1986). The atmospheric transport potential for water vapour and dust in the Sahel region. Geo Journal, 12:387-398. 10.1007/BF00262361.

Thompson, M. (1996). A standard land-cover classification scheme for remotesensingapplications in South Africa. South African Journal Of Science, 92(1):34-42.

Trollope, W. S. W. (1990). Veld management with specific reference to game ranching in the grassland and savanna areas of South Africa. Koedoe : African Protected Area Conservation and Science, 33:77-87.

Trollope, W. S. W. and Potgieter, A. L. F. (1986). Estimating grass fuel loads with a disc pasture meter in the Kruger national park. Journal of the Grassland Society of Southern Africa, 3(4):148-152.

Trollope, W. S. W., Potgieter, A. L. F., and Zambatis, N. (1989). Assessing veld condition in the Kruger national park using key grass species. Koedoe: African Protected Area Conservation and Science, 32:67-93.

Tum, M. (2012). Modelling and Validation of agricultural and forest biomass potentials for Germany and Austria. PhD thesis. Georg-August-Universität Göttingen.

Tum, M., Borg, E. (2012). A Conceptual Remote Sensing based Interception-Infiltration Model for Regional and Global Applications, Hydrology and Earth System Sciences, under review

Turner, D. P., Ritts, W. D., Cohen, W. B., Gower, S. T., Running, S. W., Zhao, M., Costa, M. H., Kirschbaum, A., Ham, J. M., Saleska, S. R. and Ahl, D. E. (2006). Evaluation of MODIS NPP and GPP products across multiple biomes. Remote Sensing of Environment, 102:282-292.

Turner, D. P., Ritts, W. D., Cohen, W. B., Maeirsperger, T. K., Gower, S. T., Kirschbaum, A. A., Running, S. W., Zhao, M., Wofsy, S. C., Dunn, A. L., Law, B. E., Campbell, J. L., Oechel, W. C., Kwon, H. J., Meyers, T. P., Small, E. E., Kurc, S. A., and Gamon, J. A. (2005). Site-level evaluation of satellite-based global terrestrial gross primary production and net primary production monitoring. Global Change Biology, 11(4):666-684. 
UNCCD (1994). Elaboration of an international convention to combat desertification in countries experiencing serious drought and/or desertification, particularly in Africa. United Nations (UN).

UNCCD (2008). The causes of desertification. Factsheet No. 2, United Nations Convention to Combat Desertification (UNCCD).

USGS (1996). GTOPO30, Global 30-Arc-Second Elevation Data Set. U.S. Geological Survey, EROS Data Center, Sioux Falls, South Dakota. http://eros.usgs.gov/\#/Find_Data/Products_And_Data_Available/GTOPO30.

Van Vegten, J. A. (1981). Man-made Vegetation Changes: an Example From Botswana's Savanna. Gaborone, Botswana: National Institute of Development and Cultural Research, Documentation Unit, University College of Botswana, University of Botswana and Swaziland.

Verger, A., Baret, F., and Weiss, M. (2011). A multisensor fusion approach to improve LAI time series. Remote Sensing of Environment, 115(10):2460 - 2470.

Verma, S. B., Baldocchi, D. D., Anderson, D. E., Matt, D. R., and Clement, R. J. (1986). Eddy fluxes of $\mathrm{CO} 2$, water vapor, and sensible heat over a deciduous forest. Boundary-Layer Meteorology, 36:71-91.

Vernekar, A. D. and Duck Chang, H. (1978). A Statistical-Dynamical Model for Stationary Perturbations in the Atmosphere. Journal of Atmospheric Sciences, 35:433-444.

Veroustraete, F., Patyn, J., and Myneni, R. B. (1996). Estimating net ecosystem exchange of carbon using the normalized difference vegetation index and an ecosystem model. Remote Sensing of Environment, 58(1):115-130.

Weiss, A. and Norman, J. (1985). Partitioning solar radiation into direct and diffuse, visible and near-infrared components. Agricultural and Forest Meteorology, 34(2-3):205 213.

Wessels, K., Prince, S., Malherbe, J., Small, J., Frost, P., and VanZyl, D. (2007). Can human-induced land degradation be distinguished from the effects of rainfall variability? A case study in South Africa. Journal of Arid Environments, 68(2):271 - 297. 
Wessels, K., Prince, S., and Reshef, I. (2008). Mapping land degradation by comparison of vegetation production to spatially derived estimates of potential production. Journal of Arid Environments, 72(10):1940 - 1949.

Wessels, K. J., Prince, S. D., Frost, P. E., and van Zyl, D. (2004). Assessing the effects of human-induced land degradation in the former homelands of northern South Africa with a $1 \mathrm{~km}$ AVHRR NDVI time-series. Remote Sensing of Environment, 91(1):47 67.

White, M., Thornton, P. E., and Running, S. W. (1997). A continental phenology model for monitoring vegetation responses to interannual climatic variability. Global Biogeochemical Cycles, 11(2):217-234.

Williams, J. R., Jones, C. A., and Dyke, P. T. (1984). A modeling approach to determining the relationship between erosion and soil productivity. Transactions of the ASABE, 27:0129-0144.

Winitzki, S. (2003). Uniform approximations for transcendental functions. In ICCSA'03 Proceedings of the 2003 international conference on Computational science and its applications: Part I.

Wisskirchen, K. (2005). Modellierung der regionalen CO2 Aufnahme durch Vegetation: $\mathrm{PhD}$ thesis, Rheinische Friedrich-Wilhelms Universität Bonn; Bonn.

Wohlfahrt, G., Bahn, M., Haubner, E., Horak, I., Michaeler, W., Rottmar, K., Tappeiner, U., and Cernusca, A. (1999). Inter-specific variation of the biochemical limitation to photosynthesis and related leaf traits of 30 species from mountain grassland ecosystems under different land use. Plant, Cell \& Environment, 22(10):1281-1296.

Woodward, F. I. (1987). Climate and Plant Distribution. Cambridge University Press, Cambridge.

Yuan, H., Dai, Y., Xiao, Z., Ji, D., and Shangguan, W. (2011). Reprocessing the modis leaf area index products for land surface and climate modelling. Remote Sensing of Environment, 115(5):1171 - 1187.

Zhao, M., Heinsch, F. A., Nemani, R. R., and Running, S. W. (2005). Improvements of the modis terrestrial gross and net primary production global data set. Remote Sensing of Environment, 95:164-175. 
Zhao, M., Running, S. W., and Nemani, R. R. (2006).Sensitivity of Moderate Resolution Imaging Spectroradiometer (MODIS) terrestrial primary production to the accuracy of meteorological reanalyses. Journal of Geophysical Research, 111: 


\section{Lebenslauf}

$\begin{array}{ll}\text { Vorame } & \text { Markus } \\ \text { Name } & \text { Niklaus } \\ \text { Adresse } & \text { Estenfelder Straße 55, 97078 Würzburg } \\ \text { Telefon } & 0179 / 9210371 \\ \text { E-Mail } & \text { markus.niklaus@web.de } \\ \text { Geburtstag } & 18.08 .1980\end{array}$

\section{Studium}

09/91 - 07/02 Alexander-von-Humboldt Gymnasium Schweinfurt

10/03 - 11/05 Grundstudium der Physik an der Julius-Maximilians-Universität Würzburg mit Nebenfach Chemie

10/05 - 11/08 Hauptstudium der Physik an der Julius-Maximilians-Universität Würzburg mit dem Abschluss als Diplom Physiker (Dipl. Phys. Univ.)

Prüfung der Angewandten Physik in „Computational Physics“

Diplomarbeit am Lehrstuhl für Astronomie mit dem Titel: „AMRKriterien in Simulationen thermischer Instabilitäten“

\section{Beruflicher Werdegang}

04/09 - 03/12 Doktorand am Deutschen Zentrum für Luft- und Raumfahrt.

Promotionsthema: Modellierung der vegetativen Produktivität zur Bewertung der Landdegradation im ariden und semi-ariden südlichen Afrika

04/12 - 03/13 Wissenschaftlicher Mitarbeiter am Deutschen Zentrum für Luft- und Raumfahrt

Würzburg, Juni 2013 



\section{Danksagung}

Diese Arbeit wurde gefördert durch das EOS Netzwerk der Helmholtz Zentren in Deutschland und am Deutschen Fernerkundungsdatenzentrum (DFD) des Deutschen Zentrums für Luft- und Raumfahrt (DLR e.V.) in Oberpfaffenhofen angefertigt.

An dieser Stelle möchte ich mich bei allen bedanken, die mich bei dieser Arbeit in unterschiedlicher Weise unterstützt haben. Zuerst gilt mein Dank Herrn Prof. Dr. Martin Kappas für die freundliche Betreuung und die unkomplizierte Hilfe bei verzwickten Problemen.

Außerdem danke ich Dr. Claudia Künzer und Dr. Kurt Günther für die intensive Begleitung und die zahlreichen wissenschaftlichen Diskussionen während der letzten Jahre.

Mein besonderer Dank gilt Christina Eisfelder und Ursula Geßner für die angenehm freundschaftliche Arbeitsatmosphäre und die zahlreichen konstruktiven Diskussionen, die diese Arbeit wesentlich verbessert haben. Außerdem danke ich allen Kolleginnen und Kollegen der Abteilung Landoberfläche für die anregende Arbeitsumgebung und das freundliche Miteinander.

Bedanken möchte ich mich auch bei den zahlreichen Korrekturlesern für die Ausdauer bei der Suche nach Fehlern und Unstimmigkeiten in meiner Arbeit. Ihr ward mir eine riesige Hilfe.

Meiner Freundin Kathrin Wolf danke ich besonders für die liebevolle Umsorgung, die immerwährende Unterstützung und die ausdauernde Geduld während der Arbeit und für den Nachdruck, diese Arbeit erfolgreich zu einem guten Ende zu bringen. Nur mit Dir als herzlichen und verständnisvollen Partner an meiner Seite war es mir möglich auch die schwierigen Zeiten während der Arbeit zu meistern.

Schließlich möchte ich mich herzlich bei meiner Familie bedanken, die mich während der Anfertigung dieser Arbeit liebevoll unterstützt haben. Meinen Eltern, Johanna und Peter Niklaus, gebührt der größte Dank, deren Hilfe und Rückhalt während des Studiums und der Promotion diese Arbeit erst möglich gemacht haben. 



\section{Erklärung}

Hiermit versichere ich, dass ich die vorliegende Arbeit selbstständig verfasst und keine anderen Hilfsmittel als die angegebenen benutzt habe.

Alle Textstellen, die wörtlich oder sinngemäß aus veröffentlichten Schriften entnommen sind, und alle Angaben, die auf mündlichen Auskünften beruhen, sind als solche kenntlich gemacht.

Würzburg, 11.01.2014 